\title{
ESTIMAÇÃO \\ E \\ TESTES DE HIPÓTESES \\ EM \\ CALIBRAÇÃO COMPARATIVA
}

Paulo Tadeu Meira e Silva de Oliveira

DISSERTAÇÃO DE MESTRADO APRESENTADA

$\mathrm{AO}$

INSTITUTO DE MATEMÁTICA E ESTATÍSTICA

DA

UNIVERSIDADE DE SÃO PAULO

PARA

OBTENÇÃO DO GRAU DE MESTRE

EM

ESTATÍSTICA

Área de Concentração: Estatística

Orientador: Prof. Dr. Heleno Bolfarine 


\title{
ESTIMAÇÃO EM TESTES DE HIPÓTESES DE CALIBRAÇÃO COMPARATIVA
}

\author{
Este exemplar corresponde \\ à redação final da \\ dissertação de mestrado devidamente corrigida \\ e defendida por \\ Paulo Tadeu Meira e Silva de Oliveira \\ e aprovada pela comissão julgadora.
}

Banca examinadora:

- Prof. Dr. Heleno Bolfarine (orientador) - IME-USP

- Prof. Dr. Mônica Carneiro Sandoval - IME - USP

- Prof. Dr. Filidor Ildifonso Vilca-Labra - IMECC - Unicamp

São Paulo, 21 de Dezembro de 2001. 


\section{QUANDO FRACASSAMOS?}

Fracassamos, Quando visualizamos a derrota, antes da batalha.

Quando abandonamos a luta, com medo da discórdia.

Quando ouvimos o Não, antes da pergunta.

Quando silenciamos, com medo da resposta.

Fracassamos,

Quando o dia amanhece, na hora que deitamos.

Quando o sono vem, e não nos entregamos.

Quando o medo de sonhar, se transforma em pesadelo. Quando a vida se dilui, na busca que fazemos.

Fracassamos, Quando a chave da verdade, abre as portas da mentira. Quando lamentamos na chegada, a hora da partida. 
Quando o Amor vai a leilão, no martelo da cobiça.

Fracassamos,

quando choramos a morte

diante da vida.

Maria Teresa Debatim

Do livro: As multas faces de um sujeito.

O que para muitos era impossível, mostrei ser possível.

Por mais difícil que seja a batalha,

Por mais difícil que seja a luta,

Não devemos desanimar,

Não devemos nos dispersar,

Devemos sempre lutar e sonhar...

Dedico esse trabalho aos meus pais,

Francisco e Geralda, que me acompanhou sempre nos bons e maus momentos, na alegria e na tristeza, na concórdia e na discórdia. 


\section{Agradecimentos}

Em primeiro lugar, o mais sincero e profundo agradecimento ao Professor Heleno Bolfarine por ter acreditado em mim, pela sugestão do tema, pelos conselhos e pela paciência ad infinitum durante a elaboração desta dissertação.

Agradecimento especial para a professora Lisbeth K. Cordani pela ajuda que prestou a mim em um dado momento no final da graduação e a professora Mônica Carneiro Sandoval pela colaboração na revisão da versão final da tese.

Outro agradecimento especial para os colegas Mário Castro, Kátia H. G. Alfaro e Daisy Gomes Tutu pela disposição, auxílio e ensino da utilização do programa Ox.

Agradeço também ao Centro Acadêmico do Instituto de Matemática e Estatística, pela permissão de utilizar o mural para a colocação de anúncio divulgando o meu trabalho e a Gorethe pela indicação de pesquisadores para que eu pudesse fazer serviço de assessoria estatística, que possibiliteou o meu sustento durante o mestrado.

Agradeço também o incentivo dos demais professores e colegas do departamento de estatística e sobretudo a minha família pelo apoio durante a realização desse trabalho.

Por fim, agradeço à Universidade de São Paulo pela oportunidade de poder melhorar a minha formação acadêmica e pelas facilidades dispensadas. 


\section{Resumo}

Sabemos da literatura que Calibração Comparativa está ligada à comparação de eficiência de instrumentos de medição.

Neste trabalho discutimos estimação e testes de hipóteses em modelos de calibração comparativa. Para a estimação dos parâmetros do modelo, consideramos os algoritmos EM e o algoritmo BFGS do programa Ox.

Testes para algumas hipóteses de interesse são implementados usando as estatísticas da razão de verossimilhanças e de Wald.

Estudos de simulação são utilizados na comparação dos procedimentos. Uma aplicação é apresentada a um conjunto de dados constituído de medições de alturas de árvores com três, quatro e cinco hipsômetros. 


\section{Abstract}

We know about the literature that Comparative Calibration it is linked the efficiency comparison of measurement instruments.

In this work we discuss estimates and tests of hypotheses in comparative calibration models. For the estimate of the parameters of the model, we consider the EM algorithm and the algorithm BFGS in the program Ox.

Testings for some hypotheses of interest are implemented using the likelihood ratio and Wald statistics.

Simulation studies are used in the comparison of the procedures. An application is presented to a data set, constituted of measurements of heights of trees with three, four and five hipsometers. 
1 de março de 2002 


\section{Sumário}

1 Introdução $\quad 2$

1.1 O Modelo de calibração comparativa . . . . . . . . . . . . . . . . . 2

1.1.1 Modelo de calibração comparativa estrutural . . . . . . . . . . . 4

1.2 O problema das alturas das árvores . . . . . . . . . . . . . . . . . 6

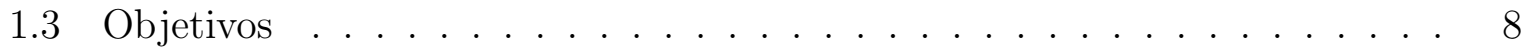

2 Estimação e testes de hipóteses no modelo de calibração comparativa 10

2.1 Introdução . . . . . . . . . . . . . . . . . . . . 10

2.2 A função de verossimilhança observada . . . . . . . . . . . . . . . . . . 10

2.3 Função de verossimilhança para os dados completos . . . . . . . . . . . . . . 11

2.4 Método dos momentos . . . . . . . . . . . . . . . . . . . . . . 11

2.5 Maximização pelo pacote $\mathrm{Ox}$. . . . . . . . . . . . . . . . 13

2.6 O algoritmo EM . . . . . . . . . . . . . . . . . . . . . 14

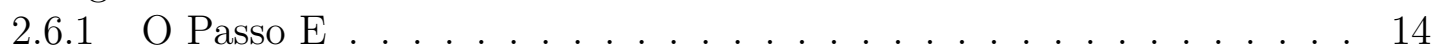

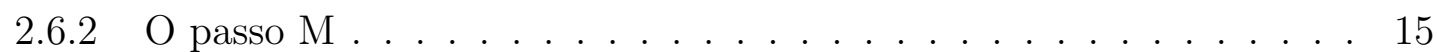

2.7 Estimador da precisão . . . . . . . . . . . . . . . . . . . . . . 16

2.8 Testes de hipóteses de interesse . . . . . . . . . . . . . . . . . . . . . . 17

2.8.1 Hipóteses de interesse . . . . . . . . . . . . . . . . . . . . . . . . . . . . . . 17

2.8.2 Teste da razão de verossimilhanças . . . . . . . . . . . . . . 17

2.8.3 Teste de Wald . . . . . . . . . . . . . . . . . . . . . . . . . 18

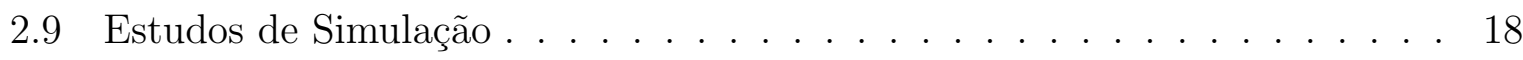

2.9.1 Algoritmo EM versus BFGS . . . . . . . . . . . . 21

3 Aplicações $\quad 27$

3.1 Introdução . . . . . . . . . . . . . . . . . . . . . . . 27

3.2 Hipóteses de Interesse . . . . . . . . . . . . . . . . . . . . . . . . 27

3.3 Análise de Variância . . . . . . . . . . . . . . . . . . . . 27

3.4 Estimadores de máxima verossimilhança . . . . . . . . . . . . . . . 28

3.5 Eficiência dos instrumentos . . . . . . . . . . . . . . . . . . . . . . . . . . . . . . . . . . . . . .

3.6 Teste de Wald . . . . . . . . . . . . . . . . . . . . . 31

3.6.1 Hipóteses de interesse . . . . . . . . . . . . . . . 56

3.6.2 Estimadores de máxima verossimilhança . . . . . . . . . . . . 57

3.7 Teste da razão de verossimilhanças . . . . . . . . . . . . . . . . . 62 
3.8 Resultados . . . . . . . . . . . . . . . . . . 62

4 Conclusões $\quad \mathbf{6 3}$

4.1 Dados reais . . . . . . . . . . . . . . . . . . . 63

4.2 Dados simulados . . . . . . . . . . . . . . . . . . . . . . . . . . . . . . . . . . . . . . . . . .

4.3 De forma geral . . . . . . . . . . . . . . . . . . 64

$\begin{array}{ll}\text { A Resultados importantes } & 65\end{array}$

$\begin{array}{ll}\text { B Função de verossimilhança para os dados observados } & 71\end{array}$

$\begin{array}{lll}\text { C } & \text { Teste da razão de verossimilhanças } & \mathbf{7 6}\end{array}$

C.1 Função de verossimilhança para os dados observados. . . . . . . . . . . . 76

C.1.1 Modelo sem restrição . . . . . . . . . . . . . . . . . . 76

C.1.2 Função de verossimilhança para a primeira hipótese de interesse . . 77

C.1.3 Razão de verossimilhanças para a primeira hipótese de interesse . . 78

C.1.4 Função de verossimilhança para a segunda hipótese de interesse . . 78

C.1.5 Razão de verossimilhanças para a segunda hipótese de interesse . 79

$\begin{array}{ll}\text { D Função de verossimilhança completa } & 80\end{array}$

E Cálculos dos valores para esperança e variância para o Modelo Completo 97

$\begin{array}{lll}\text { F } & \text { Matriz de informação esperada. } & 101\end{array}$

G Programas utilizados. $\quad 104$ 


\section{Capítulo 1}

\section{Introdução}

Neste trabalho discutimos a eficiência de algoritmos de estimação dos parâmetros do modelo e testes para quantidades como viéses dos instrumentos de medição.

\subsection{O Modelo de calibração comparativa}

Muitas medidas podem ser feitas em processos ou indivíduos, sendo que o verdadeiro valor medido é desconhecido. O problema de comparar medições quando os dados resultam de dois ou mais métodos de medição se chama calibração comparativa, isto é, calibração comparativa é a comparação de instrumentos de medição quando cada um deles é usado para medir uma característica de um grupo de unidades experimentais (Fuller, 1987). Essas unidades podem ser originárias de uma população homogênea ou de uma população dividida em estratos ou subgrupos. As comparações requeridas podem ser de dois tipos: uma relativa à calibração dos instrumentos e outra sobre a consistência ou exatidão relacionada aos instrumentos.

Neste trabalho consideramos o problema de calibração, onde $p$ instrumentos de medição são utilizados para medir a mesma quantidade desconhecida $x$, que é comum para um conjunto de $n$ indivíduos. A principal suposição é a de que existe um instrumento padrão que mede a quantidade desconhecida (não observada), sem vício, que é a medida correta e que todos os métodos de medição para os $p$ instrumentos possuem erros independentes e normalmente distribuídos.

Um modelo relacionando a quantidade desconhecida $x$ aos valores observados obtidos com os vários instrumentos foi inicialmente proposto por Barnett (1969).

Supondo que temos $p \geq 2$ instrumentos para medir uma característica ou resposta em um grupo de $n$ unidades experimentais, o modelo de calibração comparativa é dado por:

$$
Y_{i j}=\alpha_{i}+\beta_{i} x_{j}+\varepsilon_{i j}
$$


onde:

- $x_{j}$ : verdadeiro valor da característica de interesse na unidade $j, j=1, \ldots, n$;

- $Y_{i j}$ : medida fornecida pelo instrumento $i$, para a característica da unidade $j, i=$ $1,2, \ldots, p, j=1, \cdots, n$;

- $\alpha_{i}$ : vício aditivo do instrumento $i$;

- $\beta_{i}$ : vício multiplicativo do instrumento $i$;

- $\varepsilon_{i j}$ : erros de medição não correlacionados com:

$$
\mathbb{E}\left(\varepsilon_{i j}\right)=0, \operatorname{Var}\left(\varepsilon_{i j}\right)=\sigma_{i}^{2}, i=1,2, \cdots, p
$$

- $\varepsilon_{i j}$ e $x_{j}$ são independentes;

- $\varepsilon_{i j} \sim N\left(0, \sigma_{i}^{2}\right)$.

A respeito da característica a ser medida no modelo (1.1), este pode ser classificado como:

- Modelo de calibração comparativa funcional, quando os $x_{j}$ são fixos no universo de referência no qual a inferência está baseada.

- Modelo de calibração comparativa estrutural, quando os $x_{j}$ são variáveis aleatórias independentes com a mesma distribuicão.

O modelo de calibração comparativa estrutural. apresenta falta de identificabilidade e diferentes soluções são apresentadas para contornar esse problema. Bolfarine e Rojas (1995) discute com detalhes este problema de falta de identificabilidade. Barnett (1969) e Bolfarine e Rojas (1995) sugerem assumir que existe um instrumento de referência que mede $x$ sem qualquer vício. Sem perda de generalidade no modelo (1.1) podemos considerar que o instrumento 1 é o instrumento de referência, isto é, $\alpha_{1}=0$ e $\beta_{1}=1$.

Neste contexto $\alpha_{i}$ e $\beta_{i}$ caracterizam a relação entre os valores dados pelo instrumento $i$ e os valores dados pelo instrumento de referência, chamado também de instrumento padrão, e as variâncias $\sigma_{i}^{2}$ avaliam a consistência da medida dos diferentes instrumentos.

O modelo de calibração comparativa funcional e estrutural para o caso $p=2$ se reduz ao caso do modelo de regressão com erros nas variáveis usual.

O modelo de calibração comparativa funcional também apresenta dificuldades como pode ser visto em Bolfarine e Rojas (1995), Cap.5 e Vilca-Labra et al. (1998). 
No modelo estrutural quando $p=3$, Barnett (1969) consegue formas explícitas para os estimadores dos parâmetros do modelo com o método dos momentos e estes estimadores são de máxima verossimilhança se todas as estimativas das variâncias são não negativas. Para $p>3$, não existem formas explícitas para os estimadores de máxima verossimilhança; portanto, Barnett (1969) propõe estimadores pelo método dos momentos considerando conjunto de três instrumentos e depois considera combinações lineares desses estimadores tal que minimizem as variâncias assintóticas. Carter (1981) estudou o modelo proposto por Barnett para $p=3$ usando estimação de máxima verossimilhança restrita, consegue vários conjuntos de estimativas e as variâncias assintóticas dos estimadores.

\subsubsection{Modelo de calibração comparativa estrutural}

O modelo de calibração comparativa estrutural é dado pelas expressões (1.1),

$$
x_{j} \sim N\left(\mu_{x}, \sigma_{x}^{2}\right), j=1, \cdots, n
$$

Assim, pode-se mostrar que:

$$
\begin{gathered}
\mathbb{E}\left(Y_{1 j}\right)=\mu_{x}, \quad \operatorname{Var}\left(Y_{1 j}\right)=\sigma_{x}^{2}+\sigma_{1}^{2}, \\
\operatorname{IE}\left(\mathrm{Y}_{i j}\right)=\alpha_{i}+\beta_{i} \mu_{x}, \quad \operatorname{Var}\left(Y_{i j}\right)=\beta_{i}^{2} \sigma_{x}^{2}+\sigma_{i}^{2}, i=2, \ldots, p, \\
\operatorname{Cov}\left(\mathrm{Y}_{1 j}, Y_{i j}\right)=\beta_{i} \sigma_{x}^{2} \quad e \quad \operatorname{Cov}\left(Y_{i j}, Y_{l j}\right)=\beta_{i} \beta_{l} \sigma_{x}^{2}, \quad i, l=2, \ldots, p .(1 .
\end{gathered}
$$

Dessa maneira é possível concluir que $\mathbf{Y}_{j}=\left(Y_{1 j}, \ldots, Y_{p j}\right)^{\prime}$ tem distribuição normal multivariada com vetor de médias $\mu_{y}$ e matriz de covariâncias $\mathrm{V}_{\mathrm{y}}$ dados por,

$$
\begin{gathered}
\mu_{y}=\left(\begin{array}{c}
\mu_{x} \\
\alpha_{2}+\beta_{2} \mu_{x} \\
\vdots \\
\alpha_{p}+\beta_{p} \mu_{x}
\end{array}\right)=\left(\begin{array}{c}
0 \\
\alpha_{2} \\
\vdots \\
\alpha_{p}
\end{array}\right)+\left(\begin{array}{c}
1 \\
\beta_{2} \\
\vdots \\
\beta_{p}
\end{array}\right) \mu_{x}, \\
V_{Y}=\left(\begin{array}{cccc}
\sigma_{x}^{2}+\sigma_{1}^{2} & \beta_{2} \sigma_{x}^{2} & \cdots & \beta_{p} \sigma_{x}^{2} \\
\beta_{2} \sigma_{x}^{2} & \beta_{2}^{2} \sigma_{x}^{2}+\sigma_{2}^{2} & \cdots & \beta_{2} \beta_{p} \sigma_{x}^{2} \\
\vdots & \vdots & \ddots & \vdots \\
\beta_{p} \sigma_{x}^{2} & \beta_{2} \beta_{p} \sigma_{x}^{2} & \cdots & \beta_{p}^{2} \sigma_{x}^{2}+\sigma_{p}^{2}
\end{array}\right)=\Sigma+\sigma_{x}^{2} \beta \beta^{\prime},
\end{gathered}
$$


onde $\beta=\left(1, \beta_{2}, \cdots, \beta_{p}\right)^{\prime}$ e $\Sigma=\operatorname{Diag}\left(\sigma_{1}^{2}, \cdots, \sigma_{p}^{2}\right)$.

O vetor de parâmetros asssociados ao modelo é, então:

$$
\theta^{\prime}=\left(\mu_{x}, \alpha_{2}, \cdots, \alpha_{p}, \beta_{2}, \cdots, \beta_{p}, \sigma_{x}^{2}, \sigma_{1}^{2}, \cdots, \sigma_{p}^{2}\right)
$$

O objetivo é fazer inferência acerca dos parâmetros em $\theta$, portanto, a implementação de procedimentos inferenciais para $\theta$ depende das estimativas das variâncias dos estimadores. No caso do modelo estrutural, a informação de Fisher é obtida em Bolfarine e Rojas (1995). A inversa dessa matriz é a matriz de covariâncias assintóticas dos estimadores de máxima verossimilhança.

Na comparação dos instrumentos de medição para avaliar a qualidade do instrumento é importante inferir sobre a confiabilidade, a precisão e a eficiência dos instrumentos.

Confiabilidade: A confiabilidade do instrumento $i$, se define como:

$$
\rho_{i}=\frac{\beta_{i}^{2} \sigma_{x}^{2}}{\beta_{i}^{2} \sigma_{x}^{2}+\sigma_{i}^{2}}, i=1, \cdots, p
$$

O coeficiente $\rho_{i}$ pode ser interpretado como a proporção da variação explicada pelo iésimo instrumento de calibração com relação a variação total e toma valores entre zero e um. Dizemos que o instrumento $i$ forneceu medidas aproximadas da característica $x$ quando $\rho_{i}$ estiver próximo de um e se $\alpha_{i}=0$ e $\beta_{i}=1$.

Precisão: Se conhecemos $\alpha_{i}$ e $\beta_{i}$ então as medidas observadas $Y_{i j}, j=1, \cdots, n$ com o instrumento $i$ pode ser calibrado como

$$
Y_{i j}^{*}=\frac{Y_{i j}-\alpha_{i}}{\beta_{i}}=x_{j}+\frac{\varepsilon_{i j}}{\beta_{i}} j=1,2, \cdots, n
$$

de modo que estas quantidades são não viesados para $x_{j}$. Os erros de medicão $\left(\varepsilon_{i j} / \beta_{i}\right)$ têm variância $\sigma_{i}^{2} / \beta_{i}^{2}$. Portanto, a precisão do instrumento $i$ é definida como o inverso da variância do erro de medição do instrumento calibrado, é definida por $\pi_{i}$ e pode ser escrita como:

$$
\pi_{i}=\frac{\beta_{i}^{2}}{\sigma_{i}^{2}}, \quad i=1,2, \cdots, p, \quad \text { com } \beta_{1}=1
$$

É importante salientar que neste trabalho vamos considerar a precisão e a eficiência dos instrumentos. 
As variâncias $\sigma_{i}^{2}$ avaliam a consistência das medidas para os diferentes instrumentos em medidas repetidas de um mesmo indivíduo e foram assumidas como constantes de indivíduo para indivíduo.

Para este trabalho foram considerados a estimação de máxima verossimilhança de parâmetros desconhecidos pelo uso do algoritmo $E M$ para o caso $p \geq 3$.

Os dados observados são definidos por $\mathbf{Y}=\left(Y_{1}, \ldots, Y_{n}\right)^{\prime}$ e os dados não observados são definidos por $x=\left(x_{1}, \ldots, x_{n}\right)^{\prime}$. Conforme veremos mais a frente, essas duas partes colocadas juntas proporcionam uma função de verossimilhança tratável.

\subsection{O problema das alturas das árvores}

Em Dendometria (Setor da ciência que estuda a altura e o diâmetro das árvores), a medição de altura de árvores pode ser necessária para determinar o volume da madeira de um povoamento florestal ou para acompanhar o desenvolvimento florestal de uma região.

Para esse problema tivemos 10 árvores com diferentes alturas, 5 hipsômetros (instrumentos utilizados para medir a altura das árvores) e 4 observadores, sendo que cada um dos observadores fez 2 medições em cada árvore com cada instrumento e desses dados é importante salientar que:

- Foram utilizados os seguintes instrumentos: Trena (instrumento padrão, uma vez que, todas as medidas obtidas por este instrumento apresentam apenas erro de mensuração e não apresentam viéses); Altímetro de Blune Leiss; Altímetro de Haga; Hipsômetro de Weise e Altímetro de Prancheta.

- Embora as determinações de volumes podem ser feitas de forma independente da altura das árvores, esta é de fundamental importância quando resultados mais rigorosos são requeridos.

- Mesmo trabalhando com hipsômetros mais precisos, Bruce e Schumacher (1950) constataram que erros entre $0,30 \mathrm{~m}$ e $0,60 \mathrm{~m}$ não são raros, mesmo sob condições favoráveis. Usando instrumentos de menor precisão esse erro pode chegar a valores entre $0,90 \mathrm{~m}$ e $1,50 \mathrm{~m}$.

- Ao executar um trabalho dessa ordem, mesmo operando cuidadosamente com instrumentos de alta precisão uma pequena inclinação na árvore pode causar erros apreciáveis.

- Conforme já foi dito no primeiro item dessa série, um dos instrumentos, a trena foi considerada como padrão e por esse motivo foi considerada como instrumento de referência, pois apresenta maior precisão nas mensurações, isto se deve ao fato de que a árvore foi cortada antes de ter a sua altura avaliada pela trena. 
Apresentamos na próxima tabela os dados considerados em Simões et al. (1967). Nesse trabalho foi feito um estudo sobre a eficiência dos instrumentos baseado em análise de variância.

A consideração do modelo (1.1) para comparar a eficiência dos instrumentos de medição utilizados para medir alturas de árvores pode ser mais interessante em vários aspectos. Um deles está relacionado com a possibilidade de estudo de viéses através dos parâmetros $\alpha_{i}$ e $\beta_{i}$ e também através das precisões, conforme definido na seção anterior.

\begin{tabular}{c|ccccc|ccccc}
\multicolumn{1}{c|}{} & \multicolumn{7}{c}{ Tabela 1.1: Medida das alturas das árvores. } \\
& \multicolumn{7}{c}{ Observador 1 } & \multicolumn{7}{c}{ Observador 2} \\
Instrumentos & B-L & H & W & Pr & Tr & B-L & H & W & Pr & Tr \\
\hline & & & & & & & & & & \\
1a & 22.3 & 23.4 & 23.5 & 22.6 & 21.4 & 22.3 & 23.2 & 22.0 & 23.0 & 21.4 \\
1b & 22.5 & 22.4 & 23.5 & 22.4 & 21.5 & 23.0 & 22.8 & 22.0 & 22.8 & 21.5 \\
2a & 20.7 & 21.5 & 21.0 & 20.6 & 19.2 & 20.6 & 21.2 & 20.0 & 21.2 & 18.9 \\
2b & 21.0 & 21.3 & 21.0 & 20.4 & 19.2 & 20.7 & 20.2 & 19.0 & 21.2 & 18.9 \\
3a & 23.6 & 23.9 & 23.5 & 22.8 & 23.3 & 23.0 & 22.5 & 23.0 & 25.0 & 23.2 \\
3b & 23.6 & 24.0 & 24.0 & 23.6 & 23.4 & 23.0 & 22.5 & 23.5 & 24.2 & 23.2 \\
4a & 21.0 & 22.2 & 20.5 & 20.4 & 20.3 & 21.0 & 20.9 & 20.5 & 21.8 & 20.2 \\
4b & 21.0 & 22.3 & 21.0 & 21.6 & 20.4 & 20.5 & 21.0 & 20.5 & 21.2 & 20.3 \\
5a & 19.3 & 21.7 & 19.5 & 21.0 & 19.9 & 20.5 & 22.0 & 21.0 & 21.4 & 19.9 \\
5b & 18.9 & 21.1 & 19.5 & 21.0 & 20.0 & 20.0 & 22.5 & 21.5 & 21.0 & 20.0 \\
6a & 20.0 & 21.0 & 19.5 & 19.4 & 19.3 & 20.0 & 21.0 & 20.0 & 20.0 & 19.2 \\
6b & 19.6 & 21.0 & 19.5 & 18.4 & 19.4 & 19.6 & 21.5 & 19.5 & 20.0 & 19.2 \\
7a & 16.3 & 17.0 & 17.5 & 17.6 & 17.5 & 17.5 & 18.0 & 18.0 & 19.0 & 17.3 \\
7b & 16.8 & 16.8 & 17.5 & 18.0 & 17.4 & 17.0 & 18.0 & 17.5 & 18.4 & 17.4 \\
8a & 14.8 & 15.4 & 14.5 & 14.0 & 14.4 & 15.0 & 14.5 & 15.0 & 15.0 & 14.3 \\
8b & 14.7 & 14.3 & 14.5 & 14.6 & 14.5 & 15.0 & 15.0 & 14.5 & 15.0 & 14.4 \\
9a & 21.2 & 22.0 & 20.0 & 20.2 & 22.0 & 19.5 & 20.0 & 20.5 & 21.4 & 21.9 \\
9b & 21.0 & 22.0 & 20.0 & 21.0 & 22.0 & 20.0 & 21.0 & 20.5 & 21.6 & 21.7 \\
10a & 14.4 & 15.0 & 14.0 & 14.4 & 14.7 & 14.5 & 15.5 & 14.0 & 14.0 & 14.7 \\
10b & 14.2 & 15.0 & 14.0 & 14.0 & 14.8 & 14.0 & 15.0 & 14.5 & 14.4 & 14.6
\end{tabular}




\begin{tabular}{|c|c|c|c|c|c|c|c|c|c|c|}
\hline \multirow[b]{2}{*}{ Instrumentos } & \multicolumn{5}{|c|}{ Observador 3} & \multicolumn{5}{|c|}{ Observador 4} \\
\hline & B-L & $\mathrm{H}$ & W & $\operatorname{Pr}$ & $\operatorname{Tr}$ & B-L & $\mathrm{H}$ & W & $\operatorname{Pr}$ & $\operatorname{Tr}$ \\
\hline $1 \mathrm{a}$ & 22.5 & 22.5 & 23.0 & 21.1 & 21.3 & 21.0 & 21.7 & 21.0 & 21.8 & 21.2 \\
\hline $1 b$ & 22.5 & 22.5 & 22.5 & 22.4 & 21.4 & 21.5 & 22.5 & 21.5 & 22.0 & 21.2 \\
\hline $2 \mathrm{a}$ & 21.5 & 21.0 & 20.5 & 19.3 & 19.2 & 21.0 & 21.5 & 21.5 & 21.0 & 18.9 \\
\hline $2 \mathrm{~b}$ & 21.0 & 21.0 & 20.5 & 19.4 & 19.2 & 21.5 & 21.7 & 21.5 & 21.0 & 18.9 \\
\hline $3 a$ & 23.0 & 23.0 & 22.5 & 21.5 & 23.2 & 22.0 & 22.0 & 22.0 & 23.0 & 23.3 \\
\hline $3 \mathrm{~b}$ & 23.2 & 23.0 & 23.0 & 22.2 & 23.2 & 22.5 & 22.7 & 22.2 & 22.5 & 23.3 \\
\hline $4 a$ & 20.5 & 21.5 & 19.5 & 19.4 & 20.3 & 21.0 & 21.5 & 21.6 & 20.5 & 20.3 \\
\hline $4 \mathrm{~b}$ & 20.7 & 22.0 & 19.5 & 19.6 & 20.3 & 21.5 & 22.0 & 21.8 & 21.0 & 20.3 \\
\hline $5 \mathrm{a}$ & 21.0 & 23.0 & 20.0 & 20.0 & 20.0 & 17.5 & 20.0 & 19.5 & 19.8 & 19.9 \\
\hline $5 b$ & 21.0 & 22.5 & 21.0 & 21.0 & 20.0 & 18.5 & 19.5 & 20.0 & 19.6 & 19.9 \\
\hline $6 \mathrm{a}$ & 19.5 & 20.5 & 19.5 & 19.0 & 19.3 & 20.5 & 20.3 & 19.5 & 20.2 & 19.3 \\
\hline $6 \mathrm{~b}$ & 19.5 & 20.2 & 20.0 & 19.0 & 19.3 & 19.5 & 21.0 & 20.0 & 19.8 & 19.3 \\
\hline $7 \mathrm{a}$ & 17.0 & 17.2 & 17.5 & 16.2 & 17.5 & 17.5 & 16.6 & 18.9 & 18.4 & 17.4 \\
\hline $7 \mathrm{~b}$ & 16.2 & 17.2 & 17.0 & 16.5 & 17.5 & 17.0 & 16.8 & 17.5 & 18.5 & 17.4 \\
\hline $8 a$ & 14.2 & 14.7 & 14.0 & 14.0 & 14.4 & 14.5 & 15.5 & 14.5 & 14.4 & 14.5 \\
\hline $8 \mathrm{~b}$ & 14.5 & 15.0 & 14.5 & 14.2 & 14.4 & 14.8 & 16.0 & 14.7 & 14.2 & 14.5 \\
\hline $9 \mathrm{a}$ & 21.0 & 22.5 & 21.5 & 21.0 & 21.9 & 20.5 & 20.7 & 21.5 & 21.0 & 22.0 \\
\hline $9 \mathrm{~b}$ & 20.5 & 22.2 & 21.5 & 20.7 & 21.9 & 21.5 & 21.0 & 21.0 & 20.5 & 22.0 \\
\hline $10 \mathrm{a}$ & 14.0 & 16.0 & 14.5 & 14.0 & 14.8 & 14.5 & 15.5 & 14.5 & 15.0 & 14.4 \\
\hline $10 \mathrm{~b}$ & 14.2 & 16.0 & 14.0 & 13.7 & 14.8 & 14.0 & 15.3 & 15.0 & 15.2 & 14.4 \\
\hline
\end{tabular}

\subsection{Objetivos}

O objetivo principal do trabalho é comparar a eficiência dos instrumentos de medição especificada através de viéses aditivos, multiplicativos e através da variância do modelo.

Para o atendimento desse objetivo, desenvolvemos um programa, utilizando o software Ox, que possibilita a utilização de $p$ instrumentos com $n$ medidas (indivíduo), cada um sendo de mesma característica ou de um mesmo grupo de indivíduos e para isso consideramos o uso do algorítmo EM que proporciona uma solução geral para a maximização da verossimilhança dos dados observados, utilizando-se os dados completos de maneira conveniente.

Uma importante característica desse algoritmo é que as estimativas das variâncias dos erros são sempre positivas, podendo ser visto como sendo um tipo de procedimento de maximização restrita.

Em seguida, calculamos a inversa da matriz de informação esperada para o modelo completo (para detalhes, vide apêndice F) do modelo completo para estimar a matriz de covariâncias dos estimadores de máxima verossimilhança, que é válida para amostras grandes.

Para resolver o problema do teste para hipóteses de interesse sobre os parâmetros 
do modelo de calibração comparativa, podemos utilizar o teste de Wald.

Um outro teste que pode ser utilizado é o teste da razão da verossimilhanças (TRV). Neste caso são calculados os estimadores de máxima verossimilhança sob o modelo geral e sob a hipótese nula.

Um outro objetivo do trabalho é verificar quais das técnicas mostra uma maior sensibilidade para detectar diferenças entre os viéses dos instrumentos, se o teste de Wald ou da razão de verossimilhanças. 


\section{Capítulo 2}

\section{Estimação e testes de hipóteses no modelo de calibração comparativa}

\subsection{Introdução}

Neste capítulo derivamos as funções de verossimilhança observada e completa para o modelo de calibração comparativa. Consideramos a obtenção dos estimadores explícitos pelo método dos momentos, a maximização da verossimilhança observada pelo BFGS do Ox, e da verossimilhança completa pelo algoritmo EM. A estimação da eficiência e da precisão bem como testes para determinadas hipóteses de interesse como razão de verossimilhanças e o teste de Wald também são considerados.

\subsection{A função de verossimilhança observada}

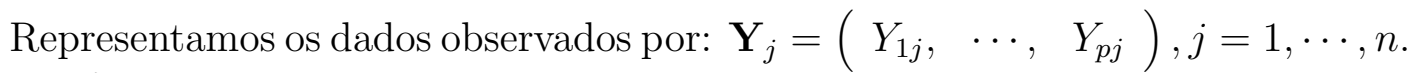
O logaritmo da função de verossimilhança para os dados observados é dada por:

$$
l\left(\theta \mid y_{1}, \cdots, y_{n}\right)=\ln (L(\theta))=-n \ln \left((2 \pi)^{\frac{p}{2}}|\Sigma|^{\frac{1}{2}}\right)-\frac{1}{2} \sum_{j=1}^{n}\left(\mathbf{Y}_{j}-\mu\right)^{\prime} \Sigma^{-1}\left(\mathbf{Y}_{j}-\mu\right)
$$

onde:

$$
\mu_{y}=\left(\begin{array}{c}
\mu_{x} \\
\vdots \\
\alpha_{p}+\beta_{p} \mu_{x}
\end{array}\right), V_{y}=\sigma_{x}^{2} \beta \beta^{\prime}+\Sigma,
$$

e $\Sigma=\operatorname{Diag}\left(\sigma_{1}^{2}, \cdots, \sigma_{p}^{2}\right)$. Para maiores detalhes a respeito da obtenção da verossimilhança acima, vide Apêndice B. 


\subsection{Função de verossimilhança para os dados com- pletos}

A função de verossimilhança completa (não observada) incorpora os dados observados e não observados.

Para os dados observados temos uma distribuição normal multivariada com vetor de médias e matriz de covariâncias, com elementos dados por: $E\left[Y_{i j}\right]=\alpha_{i}+\beta_{i} \mu_{x}$, $\operatorname{Var}\left[Y_{i j}\right]=\beta_{i}^{2} \sigma_{x}^{2}+\sigma_{i}^{2}$, e $\operatorname{Cov}\left[Y_{i j}, Y_{k j}\right]=\sigma_{x}^{2} \beta_{i} \beta_{k}, i \neq k=1, \ldots, n$.

Conforme já foi visto anteriormente, se $\mathbf{Z}_{j}=\left(\mathbf{x}_{j}, \mathbf{Y}_{j}\right)$ então, $\mathbb{E}\left[\mathbf{Z}_{j}\right]=\left(\begin{array}{l}\mu_{x} \\ \mu_{y}\end{array}\right)=$ $\mu_{z}$ e $\operatorname{Var}\left[\mathbf{Z}_{j}\right]=\Sigma_{z}=\sigma_{x}^{2} W, j=1, \ldots, n$,

$$
W=\left(\begin{array}{cc}
1 & \beta^{\prime} \\
\beta & \beta \beta^{\prime}+A
\end{array}\right) \text {. e } A=\operatorname{diag}\left(\frac{\sigma_{1}^{2}}{\sigma_{x}^{2}}, \ldots, \frac{\sigma_{p}^{2}}{\sigma_{x}^{2}}\right)
$$

Assim, o logaritmo da função de verossimilhança completa para o vetor de

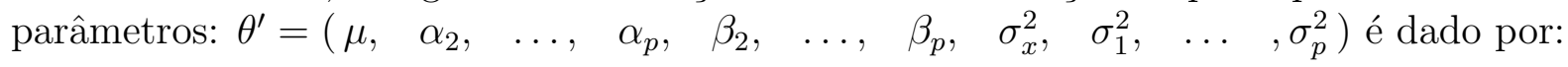

$$
l\left(\theta \mid z_{1}, \ldots, z_{n}\right)=-n \ln (2 \pi)-\frac{n}{2} \ln \left|\Sigma_{z}\right|-\frac{1}{2} \sum_{j=1}^{n}\left(\mathbf{z}_{\mathbf{j}}-\mu_{\mathbf{z}}\right)^{\prime} \Sigma_{z}^{-1}\left(\mathbf{z}_{\mathbf{j}}-\mu_{\mathbf{z}}\right),
$$

onde $\left|\Sigma_{z}\right|=\left|\sigma_{x}^{2} \mathbf{W}\right|$.

Para mais detalhes a respeito da função de verossimilhança para dados completos vide o Apêndice D.

\subsection{Método dos momentos}

Nesta seção, propomos estimar os parâmetros do modelo (1.3), com $p>3$, pelo método dos momentos, de modo que precisamos obter os momentos populacionais e amostrais do vetor aleatório $\mathbf{Y}_{j}$.

Os momentos populacionais são dados em (1.4) e os momentos amostrais são dados a seguir.

\section{Momentos amostrais}

- Médias amostrais por instrumento.

$$
\bar{Y}_{i}=\frac{\sum_{j=1}^{n} Y_{i j}}{n}
$$


- Variâncias e covariâncias amostrais.

$$
S_{Y_{i} Y_{i}}=\frac{\sum_{j=1}^{n}\left(Y_{i j}-\bar{Y}_{i}\right)^{2}}{n},
$$

e

$$
S_{Y_{i j} Y_{i^{\prime} j}}=\frac{\sum_{j=1}^{n}\left(Y_{i j}-\bar{Y}_{i}\right)\left(Y_{i^{\prime} j}-\bar{Y}_{i^{\prime}}\right)}{n}, i, i^{\prime}=1, \cdots, p ; j=1, \cdots, n .
$$

Igualando-se as médias, variâncias e covariâncias amostrais com as respectivas esperanças, variâncias e covariâncias populacionais, obtemos as seguintes equações de estimação:

$$
\begin{array}{ccc}
\hat{\mu}_{x}=\bar{Y}_{1} ; & \hat{\sigma}_{x}^{2}+\hat{\sigma}_{1}^{2}=S_{y_{1} y_{1}} ; & \hat{\beta}_{2} \hat{\sigma}_{x}^{2}=S_{y_{1} y_{2}} ; \\
\hat{\alpha}_{2}+\hat{\beta}_{2} \hat{\mu}_{x}=\bar{Y}_{2} ; & \hat{\beta}_{2}^{2} \hat{\sigma}_{x}^{2}+\hat{\sigma}_{2}^{2}=S_{y_{2} y_{2}} ; & \hat{\beta}_{3} \hat{\sigma}_{x}^{2}=S_{y_{1} y_{3}} ; \\
\hat{\alpha}_{3}+\hat{\beta}_{3} \hat{\mu}_{x}=\bar{Y}_{3} ; & \hat{\beta}_{3}^{2} \hat{\sigma}_{x}^{2}+\hat{\sigma}_{3}^{2}=S_{y_{3} y_{3} ;} ; & \vdots \\
\vdots & \vdots & \hat{\beta}_{p} \hat{\sigma}_{x}^{2}=S_{y_{1} y_{p}} ; \\
\hat{\alpha}_{p}+\hat{\beta}_{p} \hat{\mu}_{x}=\bar{Y}_{p} ; & \hat{\beta}_{p}^{2} \hat{\sigma}_{x}^{2}+\hat{\sigma}_{p}^{2}=S_{y_{p} y_{p}} ; & \hat{\beta}_{i} \hat{\beta}_{j} \hat{\sigma}_{x}^{2}=S_{y_{i} y_{j}} ; \\
i, j=2, \cdots, p ; i \neq j &
\end{array}
$$

Para $\mathrm{p}=3$ obtemos os seguintes estimadores:

$$
\begin{array}{ccc}
\hat{\mu}=\bar{Y}_{1}, & \hat{\alpha}_{2}=\bar{Y}_{2}-\hat{\beta}_{2} \bar{Y}_{1}, \quad \hat{\alpha}_{3}=\bar{Y}_{3}-\hat{\beta}_{3} \bar{Y}_{1}, \\
\hat{\sigma}_{1}^{2}=S_{Y_{1} Y_{1}}^{2}-\hat{\sigma}_{x}^{2}, & \hat{\sigma}_{2}^{2}=S_{Y_{2} Y_{2}}^{2}-\hat{\beta}_{2}^{2} \hat{\sigma}_{x}^{2}, & \hat{\sigma}_{3}^{2}=S_{Y_{3} Y_{3}}^{2}-\hat{\beta}_{3}^{2} \hat{\sigma}_{x}^{2}, \\
\hat{\beta}_{2}=\frac{S_{Y_{2} Y_{3}}}{S_{Y_{1} Y_{3}}}, & \hat{\beta}_{3}=\frac{S_{Y_{2} Y_{3}}}{S_{Y_{1} Y_{2}}}, & \hat{\sigma}_{x}^{2}=\frac{S_{Y_{1} Y_{2}} S_{Y_{1} Y_{3}}}{S_{Y_{2} Y_{3}}}
\end{array}
$$


É importante salientar que para $p>3$ os estimadores obtidos através método de momentos não são únicos, ou seja, resolvendo-se as equações acima, poderemos obter vários estimadores para um mesmo parâmetro. Mas, esses estimadores podem ser utilizados como valores iniciais para estimar os parâmetros usando o método de máxima verossimilhança, via algoritmo EM (Dempster et al, 1977).

\subsection{Maximização pelo pacote $\mathrm{Ox}$}

A rotina $M a x B F G S$ do pacote $O x$ maximiza uma função usando o método quase-Newton desenvolvido por Broyden, Fletcher, Shanno (BFGS) (ver Dornik, J.A., 1996).

Este método pode usar primeiras derivadas analíticas ou as primeiras derivadas numéricas e o processo de iteração não é influenciado pela escolha destas derivadas.

Nesta subrotina, o método quase Newton é usado(ver Gill et al., 1981) para maximizar a função $f(\theta)$, ou seja:

$$
\theta(k+1)=\theta(k)+s(k) Q(k)^{-1} q(k),
$$

onde:

- $\theta(k)$ : valores das estimativas do parâmetro na iteração $k$,

- $s(k)$ : tamanho do passo, que normalmente é 1 ,

- $\mathrm{Q}(k)$ : matriz simétrica positiva definida,

- $\mathrm{q}(k)$ : primeira derivada da função (vetor escore),

O método BFGS avalia e atualiza diretamente $\mathrm{H}=\mathrm{Q}^{-1}$. Esse método é parecido com o método de Newton exceto que a inversa da matriz hessiana $(\mathrm{G}(k))^{-1}$ é aproximada por uma matriz simétrica positiva definida $\mathrm{H}(k)$ que é corrigida e atualizada para cada iteracão.

Dois critérios de convergência são possíveis. Um deles baseado no vetor escore $q(k)$ (nulo quando avaliado no máximo) e o outro no vetor $\delta(k)$, que deve ser pequeno quando próximo do máximo.

- $\gamma(k)=q(k)-q(k-1)$ : mudança no escore, 
- $\delta(k)=\theta(k)-(k-1)$ : mudança no parâmetro,

É importante salientar que a matriz quase hessiana não pode ser usada para determinar os desvios padrões. Portanto, para obter a matriz de informação observada usamos outra sub-rotina do programa $O x$ chamada Num2Derivative, que fornece os valores da segunda derivada em relação às estimativas dos parâmetros.

No nosso caso, utilizamos esse método para calcular os valores para o vetor de parâmetros para a função observada.

\subsection{O algoritmo EM}

O algoritmo EM é utilizado para resolver um problema de estimação que é difícil de ser implementado, ou seja, a função de verossimilhança observada é difícil de ser maximizada, mas os dados observados $\left(Y_{j}\right)$ podem ser vistos como uma funcão de alguma variável não observada $\left(x_{j}\right)$ sob a qual a avaliacão dos estimadores de máximo verossimilhanca teria sido direta. A funcão de verossimilhanca completa dos dados em (2.1) é uma família exponencial regular, o que simplifica a implementacão do algoritmo $E M$.

Para ser possível a implementação do algoritmo EM, foi feita a derivação da função de verossimilhança completa (não observada, vide Apêndice D). Esta derivada incorpora os dados observados e os dados não observados, que são denotados por: $\mathbf{Y}_{\mathbf{j}}=$ $\left(\mathrm{Y}_{1 \mathrm{j}}, \ldots, \mathrm{Y}_{\mathrm{pj}}\right)^{\prime}$ e $\mathbf{x}=\left(\mathbf{x}_{\mathbf{1}}, \ldots, \mathbf{x}_{\mathbf{n}}\right)^{\prime}$, respectivamente. Essas duas partes juntas proporcionam uma função de verossimilhança tratável.

Temos que a função de verossimilhança completa (distribuição normal multivariada) é da família exponencial usual. Portanto, na etapa E basta calcular a esperança das estatísticas suficientes com relação a variável não observada obtidas da função de verossimilhança.

A etapa M é implementada maximizando-se o logaritmo da função de verossimilhança completa definida anteriormente.

\subsubsection{O Passo E}

O passo E do algoritmo determina a esperança da função de verossimilhança dos dados não observados dado os dados observados. Mas desde que estamos lidando com famílias exponenciais regulares basta calcular os valores esperados das estatísticas suficientes que seguem da função de verossimilhança observada. Consequentemente, desde

que as estatísticas suficientes só dependem de $x=\left(x_{1}, \ldots, x_{n}\right)^{\prime}$ através de $x_{j}$ e $x_{j}^{2}$ o passo $E$ é implementado calculando-se:

$$
\hat{x}_{j}=\mathbb{E}\left[x_{j} \mid Y_{j}, \theta\right]=\mu_{x}+\frac{1}{c}\left[\frac{\sigma_{x}^{2}}{\sigma_{1}^{2}} Y_{1 j}-\mu_{x}(c-1)+\beta_{*}^{\prime} A_{1}^{-1}\left(Y_{1 j}-\alpha\right)\right]
$$




$$
\operatorname{Var}\left[x_{j} \mid Y, \theta\right]=\frac{\sigma_{x}^{2}}{c}
$$

que leva a

$$
\hat{x_{j}^{2}}=\mathbb{E}\left[x_{j}^{2} \mid Y_{j}, \theta\right]=\hat{x}_{j}^{2}+\frac{\sigma_{x}^{2}}{c}
$$

onde:

$$
c=1+\sigma_{x}^{2} \sum_{i=1}^{n} \frac{\beta_{i}^{2}}{\sigma_{i}^{2}} \text { e } A_{2}=\operatorname{diag}\left(\frac{\sigma_{2}^{2}}{\sigma_{x}^{2}}, \ldots, \frac{\sigma_{p}^{2}}{\sigma_{x}^{2}}\right) .
$$

\subsubsection{O passo $M$}

O passo M do algoritmo é aquele que obtém o próximo valor dos parâmetros desconhecidos maximizando a verossimilhança dos dados com as estatísticas suficientes substituídas pelos valores esperados obtidos no passo E. Como mostrado em Dempster et al, (1977), cada passo do algorítmo incrementa a verossimilhança observada. Diferenciando o logarítmo da função de verossimilhança observada chegamos nas seguintes equações:

$$
\begin{gathered}
\hat{\mu}_{x}=\frac{1}{n} \sum_{j=1}^{n} x_{j}=\bar{x}, \\
\hat{\alpha}_{i}=\bar{Y}_{i}-\bar{x} \hat{\beta}_{i},
\end{gathered}
$$




$$
\begin{gathered}
\hat{\beta}_{i}=\frac{\sum_{j=1}^{n}\left(Y_{i j}-\bar{Y}_{i}\right)\left(x_{j}-\bar{x}\right)}{\sum_{j=1}^{n}\left(x_{j}-\bar{x}\right)^{2}}, \\
\hat{\sigma}_{x}^{2}=\frac{1}{n} \sum_{j=1}^{n}\left(x_{j}-\bar{x}\right)^{2}, \\
\hat{\sigma}_{1}^{2}=\frac{1}{n} \sum_{j=1}^{n}\left(x_{j}-Y_{1 j}\right)^{2},
\end{gathered}
$$

$$
\hat{\sigma}_{i}^{2}=\frac{1}{n} \sum_{j=1}^{n}\left(Y_{i j}-\bar{Y}_{i}\right)^{2}-\frac{1}{n} \hat{\beta}_{i}^{2} \sum_{j=1}^{n}\left(x_{j}-\bar{x}\right)^{2}=\frac{1}{n} \sum_{j=1}^{n}\left(Y_{i j}-\bar{Y}_{i}-\hat{\beta}_{i}\left(x_{j}-\bar{x}\right)\right)^{2},
$$

com $i=1, \ldots, p$.

Assim, os ciclos do algoritmo EM entre (2.5) - (2.6) e (2.9) - (2.14) seguem até a convergência quando ocorre. Conforme provado em Dempster et al. (1977) cada iteração do algoritmo aumenta a verossimilhança observada. Note que os estimadores para $\alpha_{i}$ e $\beta_{i}$ são estimadores de mínimos quadrados que dependem de $x_{j}$, que não é observado, $j=1, \ldots, n$.

\subsection{Estimador da precisão}

Um índice muito usado para avaliar a qualidade de um instrumento é a sua precisão (Alfaro, 2000) definida na seção 1.1.1.

Se $\pi_{i}=\pi_{1}, i \neq l ; i, l=1, \cdots, p$, então, dizemos que os instrumentos $i$ e $l$ medem a quantidade de interesse com a mesma precisão. Contudo os parâmetros são desconhecidos e devem ser substituídos por estimativas consistentes.

Usando a propriedade da invariância dos estimadores de máxima verossimilhança podemos obter o estimador de máxima verossimilhança(EMV) de $\pi_{i}$, isto é,

$$
\hat{\pi}_{i}=\frac{\hat{\beta}_{i}^{2}}{\hat{\sigma}_{i}^{2}}, i=1, \cdots, p
$$




\subsection{Testes de hipóteses de interesse}

\subsubsection{Hipóteses de interesse}

Comparar instrumentos de medição significa na realidade fazer inferência sobre os parâmetros que medem os vícios aditivos e multiplicativos conjuntamente ou separadamente, dependendo do interesse. Assim, podemos considerar testes para hipóteses como:

- caso $1-\mathrm{H}_{0}: \alpha_{2}=\ldots=\alpha_{p}=0$ e $\beta_{2}=\ldots=\beta_{p}=1$, versus $H_{a}$ : existe pelo menos uma diferença.

- caso $-2-\mathrm{H}_{0}: \alpha_{2}=\ldots=\alpha_{p}=\alpha$ e $\beta_{2}=\ldots=\beta_{p}=\beta$, versus $H_{a}$ : existe pelo menos uma diferença.

Note que estas hipóteses podem ser escritas como: $H_{0}: A \theta=\mathbf{b}$, para algum vetor $\mathbf{b}$, ou seja, tem uma representação na forma linear.

No teste de hipótese para o caso 1 o objetivo é verificar se os $p$ instrumentos medem a característica sem vício, enquanto que no caso 2, o objetivo é verificar se os viéses são iguais para todos os instrumentos.

\subsubsection{Teste da razão de verossimilhanças}

Um teste que pode ser utilizado neste problema é o teste de razão de verossimilhança que é largamente aplicável como a estimação de máxima verossimilhança.

A desvantagem do teste da razão de verossimilhanças é que requer estimadores de máxima verossimilhança dos parâmetros sob o modelo geral e sob a hipótese nula, para o qual precisamos de um processo iterativo, uma vez que, em geral, não é possível obter expressões explícitas para estas estimativas sob a hipótese nula.

A função de verossimilhança observada está apresentada no Apêndice B. Para testar $H_{0}: \theta \in \Theta_{0}$ versus $H_{a}: \theta \in \Theta_{0}^{c}$ calculamos a estatística (Casella e Berger, 1990):

$$
\lambda(x)=\frac{\sup _{\Theta_{0}} L(\theta / x)}{\sup _{\Theta} L(\theta / x)} .
$$

Os testes de razão de verossimilhanças são úteis também em situações onde existem complicações paramétricas, isto é, paramêtros que estão presentes no modelo, mas não são de interesse direto em termos inferenciais.

$$
\text { Para } n \rightarrow \infty,-2 \ln \lambda(x) \longrightarrow \chi_{s}^{2}
$$

Onde s é obtido através da diferença entre a quantidade de parâmetros existente no modelo geral e a quantidade de parâmetros existente no modelo restrito. 


\subsubsection{Teste de Wald}

Como, para $n$ grande, $\hat{\theta} \sim N\left(\theta, I_{F}^{-1}(\theta)\right)$, podemos utilizar a estatística de Wald que é dada por:

$$
W=(A \hat{\theta}-\mathbf{b})^{\prime}\left[A \hat{I}_{F}^{-1}(\hat{\theta}) A^{\prime}\right]^{-1}(A \hat{\theta}-\mathbf{b})
$$

Para $n$ grande $W \sim \chi_{g}^{2}$, onde $g$ é o número de graus de liberdade é equivalente a quantidade de linhas da matriz $\mathrm{A}$. Então $\mathrm{H}_{0}$ será rejeitada se $\mathrm{W} \geq \chi_{g(\alpha)}^{2}$ é obtido na Tabela de distribuição qui-quadrado com $g$ graus de liberdade que deixa, $\alpha \cdot 100 \%$ de area a sua direita.

A vantagem do teste de Wald em relação ao teste da razão de verossimilhanças é a seguinte:

- mais simples do ponto de vista computacional, pois utiliza apenas os estimadores dos parâmetros sob o modelo geral.

\subsection{Estudos de Simulação}

Nesta seção apresentamos um estudo de simulação utilizando o software OX para $p=3$ instrumentos com a quantidade de observações em cada instrumento variando de $n=10$ a $n=50$ e a quantidade de simulações variando entre 100 a 5000 em cada uma dessas situações descritas acima, temos o modelo de calibração comparativa descrito no capítulo 1 com $x_{j} \sim N(50,100) ; \varepsilon_{1}, \varepsilon_{2}$ e $\varepsilon_{3} \sim N(0,25) ; \alpha_{1}=\alpha_{2}=\alpha_{3}=0$; $\beta_{1}=\beta_{2}=\beta_{3}=1$ e $j=1, \cdots, n$.

As amostras foram geradas da seguinte maneira:

- Inicialmente foram geradas 100 amostras dos erros $\varepsilon_{i j}$ com as distribuições acima e a seguir foram geradas $x_{i j}$ e $y_{i j}$ usando os valores de $\alpha_{i}, \beta_{i}, \mu_{x}, \sigma_{x}^{2}$ e $\sigma_{i}^{2}, i=1,2,3$ considerados acima;

- Todo esse processo foi repetido para tamanhos de amostras $n=20,30,40$ e 50 e quantidades de simulações iguais a 500, 1000, 2000, 3000, 4000 e 5000.

- objetivos da simulação: Teste de hipóteses

As hipóteses de interesse que foram consideradas são:

$$
H_{01}: \alpha_{2}=\alpha_{3}=0, \beta_{2}=\beta_{3}=1
$$

e

$$
H_{02}: \alpha_{2}=\alpha_{3}=\alpha, \beta_{2}=\beta_{3}=\beta
$$


Para cada simulação foram calculadas a estatística de Wald e da razão de verossimilhanças.

\section{Teste de Wald}

Para cada simulação foram obtidos:

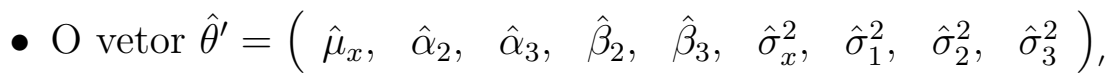

- A matriz de informação de Fisher que foi obtida de acordo com as fórmulas mencionadas no Apêndice F.

- Em seguida foi calculada a inversa dessa matriz, que é uma matriz de covariâncias a ser usada na estatística de Wald.

Note que $H_{01}: \alpha_{2}=\alpha_{3}=0, \beta_{2}=\beta_{3}=1$, pode ser escrita na forma $H_{01}: A \theta=\mathbf{b}$; com a matriz $\mathrm{A}$ e vetor $\mathbf{b}$ dados por:

$$
A=\left(\begin{array}{ccccccccc}
0 & 1 & 0 & 0 & 0 & 0 & 0 & 0 & 0 \\
0 & 0 & 1 & 0 & 0 & 0 & 0 & 0 & 0 \\
0 & 0 & 0 & 1 & 0 & 0 & 0 & 0 & 0 \\
0 & 0 & 0 & 0 & 1 & 0 & 0 & 0 & 0
\end{array}\right) \text { e } \mathbf{b}=\left(\begin{array}{c}
0 \\
0 \\
1 \\
1
\end{array}\right)
$$

de modo que $A \hat{\theta}=\left(\begin{array}{llll}\hat{\alpha}_{2}, & \hat{\alpha}_{3}, & \hat{\beta}_{2}, & \hat{\beta}_{3}\end{array}\right)^{\prime}$.

Para $\mathrm{H}_{01}$, o número de graus de liberdade é 4 .

Para verificar se esses vícios aditivos e multiplicativos são todos iguais entre eles, a hipótese de interesse é dada por: $H_{02}: \alpha_{2}=\alpha_{3}=\alpha, \beta_{2}=\beta_{3}=\beta$; onde:

$$
A=\left(\begin{array}{ccccccccc}
0 & 1 & -1 & 0 & 0 & 0 & 0 & 0 & 0 \\
0 & 0 & 0 & 1 & -1 & 0 & 0 & 0 & 0
\end{array}\right) \text { e } \mathbf{b}=\left(\begin{array}{c}
0 \\
0
\end{array}\right), \operatorname{com} A \hat{\theta}=\left(\begin{array}{c}
\hat{\alpha}_{2}-\hat{\alpha}_{3} \\
\hat{\beta}_{2}-\hat{\beta}_{3}
\end{array}\right) .
$$

O valor observado da estatística com o valor crítico obtido a partir da distribuição qui-quadrado com 4 graus de liberdade e nível de significância de 5\%, para $\mathrm{H}_{01}$.

Para $\mathrm{H}_{02}$, o número de graus de liberdade é 2 .

- determinar o número de vezes em que um determinado instrumento é mais eficiente que os demais.

- determinar o número de vezes em que um determinado instrumento é mais preciso que os demais.

A Tabela 2.1 apresenta a porcentagem de vezes em que um determinado instrumento foi mais eficiente em relação a certo número de simulações e tamanho de amostra. 
Tabela 2.1 - Quantidade de simulações em que um determinado instrumento foi mais eficiente entre os três instrumentos(\%).

\begin{tabular}{|c|c|c|c|c|c|c|c|c|c|c|c|c|c|c|c|}
\hline \multirow{2}{*}{$\begin{array}{l}\text { quantidade } \\
\text { de } \\
\text { simulações }\end{array}$} & \multicolumn{3}{|c|}{$\begin{array}{c}\mathrm{n}=10 \\
\text { instrumentos }\end{array}$} & \multicolumn{3}{|c|}{$\begin{array}{c}\mathrm{n}=20 \\
\text { instrumentos }\end{array}$} & \multicolumn{3}{|c|}{$\begin{array}{c}\mathrm{n}=30 \\
\text { instrumentos }\end{array}$} & \multicolumn{3}{|c|}{$\begin{array}{c}\mathrm{n}=40 \\
\text { instrumentos }\end{array}$} & \multicolumn{3}{|c|}{$\begin{array}{c}\mathrm{n}=50 \\
\text { instrumentos }\end{array}$} \\
\hline & 1 & 2 & 3 & 1 & 2 & 3 & 1 & 2 & 3 & 1 & 2 & 3 & 1 & 2 & 3 \\
\hline 100 & 35 & 34 & 3 & & 26 & 3 & & 38 & 32 & & & 32 & & 40 & 28 \\
\hline & & 34.8 & 32 & & 32.2 & 36 . & & & 32 & & 35.2 & 32 & & 37 & 31.2 \\
\hline & 35.9 & 31.7 & 32.4 & 34.4 & 33.6 & 32 & 32.3 & 35.7 & 32 & 32.9 & 35.3 & 31.8 & 33.3 & 34.5 & 32.2 \\
\hline & 36.1 & 31.5 & 32.5 & 34.1 & 33.4 & 32.8 & 32 & 34.4 & 33. & 34 & 34.2 & 31.9 & 33.8 & 34.1 & 32.1 \\
\hline & 35.3 & 31.8 & 32.9 & 33.7 & 33.4 & 33 & 32.4 & 34.4 & 33 & 34 & 33.7 & 32.3 & 33.5 & 33.4 & 33.1 \\
\hline & 34.5 & 32.9 & 32.6 & 33 & 33.7 & 33.4 & 32.3 & 34.1 & 33.6 & 34.3 & 33.2 & 32.5 & 33.2 & 33.5 & 33.4 \\
\hline 5000 & 34.4 & 32.8 & 32.8 & 32.8 & 33.6 & 33.6 & 32 & 34.1 & 33.9 & 33.8 & 33.2 & 33 & 33.4 & 33.5 & 33.1 \\
\hline
\end{tabular}

A Tabela 2.2 apresenta as mesmas quantidades para o caso da precisão.

Observando as tabelas de eficiência e de precisão foi possível observar que, quanto maior a quantidade de observações, as proporções tendem ao equilíbrio, isto é, a porcentagem de vezes em que um determinado instrumento é mais eficiente e mais preciso que os demais, tende a se igualar aos demais.

Tabela 2.2 - Quantidade de simulações em que um determinado instrumento foi mais preciso entre os três instrumentos(\%).

\begin{tabular}{|c|c|c|c|c|c|c|c|c|c|c|c|c|c|c|c|}
\hline \multirow{2}{*}{$\begin{array}{l}\text { quantidade } \\
\text { de } \\
\text { simulações }\end{array}$} & \multicolumn{3}{|c|}{$\begin{array}{c}\mathrm{n}=10 \\
\text { instrumentos }\end{array}$} & \multicolumn{3}{|c|}{$\begin{array}{c}\mathrm{n}=20 \\
\text { instrumentos }\end{array}$} & \multicolumn{3}{|c|}{$\begin{array}{c}\mathrm{n}=30 \\
\text { instrumentos }\end{array}$} & \multicolumn{3}{|c|}{$\begin{array}{c}\mathrm{n}=40 \\
\text { instrumentos }\end{array}$} & \multicolumn{3}{|c|}{$\begin{array}{c}\mathrm{n}=50 \\
\text { instrumentos }\end{array}$} \\
\hline & 1 & 2 & 3 & 1 & 2 & 3 & 1 & 2 & 3 & 1 & 2 & 3 & 1 & 2 & 3 \\
\hline 100 & 25 & 42 & 33 & 36 & 23 & 41 & 29 & 35 & 36 & & 34 & 30 & 29 & 44 & 27 \\
\hline & 29 & 38.8 & 32 & & 32 & & & 34 & 34 & & 34.8 & 33.8 & 9.6 & 38.2 & 32.2 \\
\hline & 1.2 & 34.1 & & & 33.9 & & & 33.9 & 34 & .6 & 35.3 & 33.1 & 1.7 & 35.2 & 33.1 \\
\hline 00 & 32 & 33.7 & 34 & 3.1 & 33.8 & 33.1 & 2.8 & 33.3 & 34 & 2.8 & 34.7 & 32.6 & 3.6 & 33.7 & 32.8 \\
\hline 000 & & 34.2 & 34.7 & 32.5 & 33.3 & 34.2 & 32.9 & 34.1 & 32.9 & 33.2 & 34.2 & 32.6 & 33.2 & 33.3 & 33.5 \\
\hline 4000 & 30.2 & 35.1 & 34.7 & 32.2 & 33.6 & 34.3 & 32.7 & 33.9 & 33.4 & 32.9 & 34 & 33.1 & 33 & 33.5 & 33.5 \\
\hline 5000 & 30.1 & 34.6 & 35.2 & 32 & 34 & 34 & 32.2 & 33.7 & 34.1 & 32.7 & 34.1 & 33.2 & 32.8 & 33.7 & 33.8 \\
\hline
\end{tabular}

Foram calculadas as estimativas dos parâmetros para todos os tamanhos de amostra e quantidades de simulações que foram consideradas para os testes de Wald e da razão da verossimilhanças para as hipóteses de interesse para a equação (2.15), cujos resultados vem na Tabela 2.4 .

Considerando a tabela 2.4, foi possível verificar que, quanto maior a quantidade de simulações e quanto maior o tamanho da amostra, mais os valores das estimativas dos parâmetros se aproximam dos valores teóricos e mais esses mesmos valores se ajustam a uma distribuição normal.

Nota-se na Tabela 2.3, que para cada tamanho de amostra (linha) e para cada quantidade de simulações (colunas) foram calculados as seguintes quantidades:

- "miss ": quantidade de simulações em que foi notada a presença de " missings" provenientes de problemas decorrentes com a inversão da matriz de informação. Este problema ocorre com maior frequência para tamanhos pequenos de amostra. 
- neg: quantidade de simulações em que foi notada a presença de valores negativos na estatística do teste de Wald devido ao fato da inversa matriz de informação apresentar valores negativos na diagonal, muito proximos de zero (da ordem de $10^{-15}$, aproximadamente).

- rej: quantidade de simulações em que foi notada a presença de valores de níveis descritivos ( $\mathrm{p}$ valor) menores que 0,05, ou seja, quantidade de simulações em que o teste foi rejeitado para cada uma das hipóteses.

- simaprov: quantidade de testes considerados, isto é, descontados os casos miss e também os valores "neg".

- prop: proporção em porcentagem, isto é, o quociente entre quantidades de rejeições (rej) e quantidade total de simulações consideradas (simaprov).

- essa rotina foi repetida para as duas hipóteses de interesse e também para os testes de Wald e teste da razão de verossimilhança.

Verificando os resultados existente na tabela 2.3, é possível verificar que a proporção de rejeições em geral; diminui a medida em que aumenta o tamanho da amostra.

O cálculo do teste da razão de verossimilhanças parece apresentar mais problemas de cálculo que o de Wald, pois temos mais "miss" que "neg", em geral.

Pode-se verificar que o teste de Wald para a segunda hipótese de interesse foi a que obteve nível empírico (prop.) mais próximo do nível teórico (5\%).

\subsubsection{Algoritmo EM versus BFGS}

O algorítmo BFGS como qualquer outro algorítmo de otimização numérica busca o ponto estacionário de uma determinada função. Ele pode ser considerado um algorítmo de quasi-Newton por não haver a necessidade de fornecer a matriz hessiana.

Esse algoritmo possui como vantagem, a não necessidade de os estimadores serem calculados de forma explícita, isto é, bastando apenas fornecer a função de verossimilhanças observada e é mais rápido em termos de tempo de processamento em relação ao algoritmo EM. Porém, possui como desvantagem, a necessidade de cálculos de inversas e determinantes de matrizes que podem acarretar problemas em certas ocasiões, principalmente com valores bem próximos de zero (da ordem de $10^{-15}$ ).

O algorítmo EM também é utilizado para determinar pontos estacionários (máximos, mínimos ou cela), porém é usado na situação em que temos variáveis latentes.

Um exemplo em que o BFGS não converge e o EM converge para $\mathrm{p}=3$ instrumentos e tamanho de amostra igual a 10 é obtido quando se considera os dados simulados abaixo. Os parâmetros usados para simular os dados são os mesmos que usamos na seção 2.8 . 


$\begin{array}{lll}42.833 & 48.977 & 39.950 \\ 33.434 & 31.059 & 26.692 \\ 49.384 & 40.638 & 37.025 \\ 44.025 & 53.836 & 50.576 \\ 47.619 & 42.388 & 41.481 \\ 34.576 & 38.913 & 35.990 \\ 42.906 & 43.227 & 45.350 \\ 57.542 & 50.429 & 47.909 \\ 26.688 & 39.953 & 41.846 \\ 46.118 & 59.380 & 57.958\end{array}$

A seguinte mensagem é obtida na saída do Ox:

Warning: invert: decomposition failed C:3sim.ox (275): main

Portanto, como conseqüência não ocorre a convergência dos estimadores no algorítmo BFGS, tornando impraticável também o cálculo do teste da razão de verossimilhança com o uso dos dados observados, enquanto que utilizando o algorítmo EM, a convergência costuma ocorrer em menos de 100 iterações.

madores:

Nesse caso em particular, obtemos pelo algorítmo EM os seguintes esti-

\begin{tabular}{|c|c|c|c|c|c|c|c|c|}
\hline$\hat{\mu}$ & $\hat{\alpha}_{2}$ & $\hat{\alpha}_{3}$ & $\hat{\beta}_{2}$ & $\hat{\beta}_{3}$ & $\hat{\sigma}_{x}^{2}$ & $\hat{\sigma}_{1}^{2}$ & $\hat{\sigma}_{2}^{2}$ & $\hat{\sigma}_{3}^{2}$ \\
\hline 42.513 & -25.846 & -26.271 & 1.6636 & 1.6171 & 22.212 & 50.183 & 0.11084 & 9.08 \\
\hline
\end{tabular}

Conforme já foi visto anteriormente, o algorítmo EM consiste de duas etapas, ou seja:

- primeira - atualização da esperança condicional,

- segunda - maximização da função de verossimilhança não observada.

Podem ocorrer então dificuldades em termos de programação, uma vez que para cada situação, pode ser necessário escrever um programa diferente, o que costuma ser mais lento em termos de tempo de processamento ao compararmos com o tempo necessário no uso do BFGS e por fim, o uso do algoritmo EM é facilitado quando temos a forma explícita para os estimadores na etapa M.

O algoritmo EM possui como vantagem uma maior confiabilidade em termos computacionais, gerando menores problemas para o seu cálculo. 
Tabela 2.3 - Resultados das simulações para as hipóteses de interesse. 
Tabelas 2.4 - Valores das estimativas dos parâmetros e dos desvios padrões para os dados simulados.

$\mathrm{n}=10$ - Estimativas dos parâmetros

\begin{tabular}{|c|c|c|c|c|c|c|c|c|c|}
\hline simulações & $\hat{\mu}_{x}$ & $\hat{\alpha}_{2}$ & $\hat{\alpha}_{3}$ & $\hat{\beta}_{2}$ & $\hat{\beta}_{3}$ & $\hat{\sigma}_{x}^{2}$ & $\hat{\sigma}_{1}^{2}$ & $\hat{\sigma}_{2}^{2}$ & $\hat{\sigma}_{3}^{2}$ \\
\hline 100 & 50.288 & 3.157 & 1.212 & 0.940 & 0.975 & 74601.18 & 73399.07 & 17.954 & 20.162 \\
500 & 52.585 & 9.573 & 4.177 & 0.812 & 0.924 & 66157.91 & 66741.23 & 18.715 & 19.010 \\
1000 & 51.560 & 59.350 & 57.320 & -0.305 & -0.264 & 57476.67 & 57915.47 & 19.449 & 19.276 \\
2000 & 51.593 & 40.655 & 38.927 & 0.1188 & 0.151 & 52576.27 & 53308.81 & 19.659 & 19.624 \\
3000 & 51.958 & 32.076 & 30.891 & 0.310 & 0.334 & 49810.59 & 50818.36 & 19.454 & 19.373 \\
4000 & 51.710 & 28.104 & 26.367 & 0.394 & 0.428 & 51427.39 & 52349.26 & 19.373 & 19.363 \\
5000 & 51.951 & 25.323 & 23.666 & 0.459 & 0.491 & 48959.58 & 49812.2 & 19.405 & 19.326 \\
\hline
\end{tabular}

$\mathrm{n}=10$ - Desvios padrões

\begin{tabular}{|c|c|c|c|c|c|c|c|c|c|}
\hline simulações & $\hat{\mu}_{x}$ & $\hat{\alpha}_{2}$ & $\hat{\alpha}_{3}$ & $\hat{\beta}_{2}$ & $\hat{\beta}_{3}$ & $\hat{\sigma}_{x}^{2}$ & $\hat{\sigma}_{1}^{2}$ & $\hat{\sigma}_{2}^{2}$ & $\hat{\sigma}_{3}^{2}$ \\
\hline 100 & 5.848 & 19.730 & 21.642 & 0.390 & 0.414 & 353239.5 & 348090.2 & 16.785 & 14.213 \\
500 & 40.367 & 239.261 & 229.122 & 4.924 & 4.817 & 366974.8 & 365804.5 & 15.940 & 14.277 \\
1000 & 37.642 & 1634.955 & 1665.087 & 36.529 & 37.213 & 313369.8 & 311891.7 & 15.003 & 14.813 \\
2000 & 40.603 & 1163.893 & 1183.885 & 25.981 & 26.441 & 309978.2 & 310122.7 & 15.398 & 15.107 \\
3000 & 43.499 & 955.851 & 973.099 & 21.315 & 21.704 & 291565.5 & 292707 & 15.100 & 14.986 \\
4000 & 42.696 & 867.662 & 884.430 & 19.307 & 19.677 & 298913 & 300724.9 & 15.093 & 14.892 \\
5000 & 41.526 & 781.493 & 795.165 & 17.365 & 17.673 & 287985.8 & 289280.6 & 15.152 & 14.849 \\
\hline
\end{tabular}

$\mathrm{n}=20$ - Estimativas dos parâmetros

\begin{tabular}{|c|c|c|c|c|c|c|c|c|c|}
\hline simulações & $\hat{\mu}_{x}$ & $\hat{\alpha}_{2}$ & $\hat{\alpha}_{3}$ & $\hat{\beta}_{2}$ & $\hat{\beta}_{3}$ & $\hat{\sigma}_{x}^{2}$ & $\hat{\sigma}_{1}^{2}$ & $\hat{\sigma}_{2}^{2}$ & $\hat{\sigma}_{3}^{2}$ \\
\hline 100 & 50.676 & 1.474 & 2.034 & 0.972 & 0.962 & 30551.05 & 29947.46 & 24.244 & 22.647 \\
500 & 49.964 & -1.057 & -1.714 & 1.018 & 1.031 & 10598.19 & 10343.5 & 22.518 & 21.624 \\
1000 & 50.0645 & 0.668 & 0.889 & 0.984 & 0.980 & 6903.325 & 6711.443 & 22.066 & 22.141 \\
2000 & 50.541 & 2.250 & 3.192 & 0.955 & 0.936 & 6529.438 & 6513.312 & 22.157 & 22.237 \\
3000 & 50.331 & 5.077 & 6.011 & 0.896 & 0.878 & 8671.478 & 8643.134 & 22.101 & 22.172 \\
4000 & 50.323 & 6.473 & 7.385 & 0.867 & 0.848 & 7738.735 & 7684.196 & 22.096 & 22.054 \\
5000 & 50.518 & 29.504 & 31.068 & 0.407 & 0.376 & 8508.282 & 8533.08 & 22.097 & 22.083 \\
\hline
\end{tabular}

$\mathrm{n}=20$ - Desvios padrões

\begin{tabular}{|c|c|c|c|c|c|c|c|c|c|}
\hline simulações & $\hat{\mu}_{x}$ & $\hat{\alpha}_{2}$ & $\hat{\alpha}_{3}$ & $\hat{\beta}_{2}$ & $\hat{\beta}_{3}$ & $\hat{\sigma}_{x}^{2}$ & $\hat{\sigma}_{1}^{2}$ & $\hat{\sigma}_{2}^{2}$ & $\hat{\sigma}_{3}^{2}$ \\
\hline 100 & 3.707 & 13.900 & 13.163 & 0.271 & 0.261 & 237597.6 & 233787.4 & 12.570 & 13.064 \\
500 & 2.849 & 49.001 & 56.284 & 0.933 & 1.052 & 129761 & 127661.8 & 12.341 & 11.534 \\
1000 & 3.245 & 59.286 & 64.062 & 1.204 & 1.289 & 96908.26 & 95379.06 & 11.989 & 11.615 \\
2000 & 13.606 & 101.236 & 101.553 & 2.049 & 2.038 & 100404.3 & 99820.08 & 11.999 & 11.716 \\
3000 & 13.794 & 144.924 & 150.838 & 3.008 & 3.118 & 121978.2 & 121383.4 & 12.025 & 11.732 \\
4000 & 12.289 & 150.985 & 154.516 & 3.139 & 3.204 & 111778.9 & 111145.6 & 11.975 & 11.739 \\
5000 & 15.513 & 1644.217 & 1691.61 & 32.695 & 33.638 & 118962.9 & 118918.4 & 11.947 & 11.738 \\
\hline
\end{tabular}


$\mathrm{n}=30$ - Estimativas dos parâmetros

\begin{tabular}{|c|c|c|c|c|c|c|c|c|c|}
\hline simulações & $\hat{\mu}_{x}$ & $\hat{\alpha}_{2}$ & $\hat{\alpha}_{3}$ & $\hat{\beta}_{2}$ & $\hat{\beta}_{3}$ & $\hat{\sigma}_{x}^{2}$ & $\hat{\sigma}_{1}^{2}$ & $\hat{\sigma}_{2}^{2}$ & $\hat{\sigma}_{3}^{2}$ \\
\hline 100 & 49.947 & -0.014 & 0.259 & 1.001 & 0.993 & 101.596 & 23.067 & 21.560 & 22.175 \\
500 & 49.931 & -1.035 & -1.193 & 1.022 & 1.024 & 97.807 & 23.923 & 23.292 & 22.721 \\
1000 & 49.922 & -1.152 & -0.556 & 1.024 & 1.011 & 106.073 & 30.601 & 22.920 & 23.366 \\
2000 & 50.288 & -1.394 & -1.312 & 1.029 & 1.027 & 2795.63 & 2824.466 & 22.885 & 23.110 \\
3000 & 50.259 & -1.046 & -0.976 & 1.021 & 1.019 & 1955.973 & 1953.668 & 22.803 & 23.265 \\
4000 & 50.197 & -0.865 & -0.866 & 1.017 & 1.017 & 1984.026 & 1954.659 & 22.750 & 23.045 \\
5000 & 50.179 & -0.791 & -0.785 & 1.016 & 1.016 & 2183.209 & 2137.705 & 22.762 & 23.075 \\
\hline
\end{tabular}

$\mathrm{n}=30$ - Desvios padrões

\begin{tabular}{|c|c|c|c|c|c|c|c|c|c|}
\hline simulações & $\hat{\mu}_{x}$ & $\hat{\alpha}_{2}$ & $\hat{\alpha}_{3}$ & $\hat{\beta}_{2}$ & $\hat{\beta}_{3}$ & $\hat{\sigma}_{x}^{2}$ & $\hat{\sigma}_{1}^{2}$ & $\hat{\sigma}_{2}^{2}$ & $\hat{\sigma}_{3}^{2}$ \\
\hline 100 & 2.008 & 7.488 & 7.837 & 0.148 & 0.158 & 34.255 & 10.808 & 9.233 & 9.304 \\
500 & 2.027 & 25.671 & 32.156 & 0.499 & 0.627 & 32. & 10.982 & 10.149 & 9.587 \\
1000 & 2.056 & 19.018 & 23.465 & 0.370 & 0.458 & 282.567 & 227.796 & 10.043 & 10.044 \\
2000 & 12.297 & 77.458 & 95.254 & 1.583 & 1.947 & 67848.26 & 70627.87 & 9.947 & 10.013 \\
3000 & 10.444 & 63.403 & 77.922 & 1.296 & 1.593 & 55479.9 & 57758.06 & 9.830 & 9.974 \\
4000 & 9.104 & 55.321 & 67.704 & 1.130 & 1.384 & 54774.8 & 56336.05 & 9.800 & 9.904 \\
5000 & 8.270 & 49.629 & 60.675 & 1.014 & 1.240 & 55894.91 & 56966.09 & 9.806 & 9.930 \\
\hline
\end{tabular}

$\mathrm{n}=40$ - Estimativas dos parâmetros

\begin{tabular}{|c|c|c|c|c|c|c|c|c|c|}
\hline simulações & $\hat{\mu}_{x}$ & $\hat{\alpha}_{2}$ & $\hat{\alpha}_{3}$ & $\hat{\beta}_{2}$ & $\hat{\beta}_{3}$ & $\hat{\sigma}_{x}^{2}$ & $\hat{\sigma}_{1}^{2}$ & $\hat{\sigma}_{2}^{2}$ & $\hat{\sigma}_{3}^{2}$ \\
\hline 100 & 49.924 & 0.110 & -0.014 & 0.996 & 1.0012 & 95.794 & 22.647 & 23.231 & 23.537 \\
500 & 50.039 & -0.460 & -0.447 & 1.008 & 1.007 & 97.921 & 23.889 & 23.095 & 23.512 \\
1000 & 49.912 & -0.751 & -0.433 & 1.014 & 1.008 & 97.278 & 23.752 & 23.205 & 23.843 \\
2000 & 49.966 & -0.960 & -0.964 & 1.019 & 1.019 & 833.9275 & 748.387 & 23.240 & 23.764 \\
3000 & 49.991 & -0.765 & -0.787 & 1.015 & 1.016 & 820.734 & 735.117 & 23.296 & 23.780 \\
4000 & 50.002 & 0.027 & 0.203 & 0.999 & 0.995 & 952.811 & 864.415 & 23.379 & 23.725 \\
5000 & 49.993 & -0.023 & 0.142 & 1. & 0.997 & 781.748 & 696.281 & 23.365 & 23.690 \\
\hline
\end{tabular}

$\mathrm{n}=40$ - Desvios padrões

\begin{tabular}{|c|c|c|c|c|c|c|c|c|c|}
\hline simulações & $\hat{\mu}_{x}$ & $\hat{\alpha}_{2}$ & $\hat{\alpha}_{3}$ & $\hat{\beta}_{2}$ & $\hat{\beta}_{3}$ & $\hat{\sigma}_{x}^{2}$ & $\hat{\sigma}_{1}^{2}$ & $\hat{\sigma}_{2}^{2}$ & $\hat{\sigma}_{3}^{2}$ \\
\hline 100 & 1.698 & 8.550 & 7.218 & 0.167 & 0.141 & 29.919 & 7.791 & 9.359 & 8.515 \\
500 & 1.784 & 7.095 & 6.810 & 0.140 & 0.134 & 28.297 & 8.232 & 9.059 & 8.9 \\
1000 & 1.775 & 6.904 & 7.062 & 0.137 & 0.140 & 27.544 & 8.596 & 8.824 & 8.777 \\
2000 & 1.754 & 22.802 & 24.462 & 0.463 & 0.498 & 32897.08 & 32415.75 & 8.839 & 8.841 \\
3000 & 2.693 & 19.105 & 20.474 & 0.388 & 0.416 & 28678.43 & 28233.78 & 8.770 & 8.798 \\
4000 & 2.485 & 45.744 & 53.396 & 0.941 & 1.099 & 31740.91 & 31225.79 & 8.845 & 8.880 \\
5000 & 2.361 & 41.473 & 48.753 & 0.853 & 1.002 & 28391.29 & 27930.52 & 8.852 & 8.767 \\
\hline
\end{tabular}


$\mathrm{n}=50$ - Estimativas dos parâmetros

\begin{tabular}{|c|c|c|c|c|c|c|c|c|c|}
\hline simulações & $\hat{\mu}_{x}$ & $\hat{\alpha}_{2}$ & $\hat{\alpha}_{3}$ & $\hat{\beta}_{2}$ & $\hat{\beta}_{3}$ & $\hat{\sigma}_{x}^{2}$ & $\hat{\sigma}_{1}^{2}$ & $\hat{\sigma}_{2}^{2}$ & $\hat{\sigma}_{3}^{2}$ \\
\hline 100 & 50.083 & -1.197 & -1.220 & 1.021 & 1.025 & 97.596 & 23.519 & 22.179 & 23.784 \\
500 & 49.9 & -0.692 & -0.509 & 1.0139 & 1.011 & 99.156 & 24.177 & 23.222 & 24.129 \\
1000 & 49.954 & -0.762 & -0.454 & 1.014 & 1.010 & 98.979 & 23.777 & 23.714 & 23.676 \\
2000 & 49.954 & -0.628 & -0.339 & 1.012 & 1.007 & 98.703 & 23.605 & 23.980 & 23.863 \\
3000 & 49.944 & -0.477 & -0.347 & 1.010 & 1.007 & 98.297 & 23.739 & 24.066 & 23.811 \\
4000 & 49.946 & -0.423 & -0.293 & 1.009 & 1.006 & 98.124 & 23.816 & 23.904 & 23.862 \\
5000 & 49.957 & -0.400 & -0.343 & 1.008 & 1.007 & 98.280 & 23.827 & 23.840 & 23.862 \\
\hline
\end{tabular}

$\mathrm{n}=50$ - Desvios padrões

\begin{tabular}{|c|c|c|c|c|c|c|c|c|c|}
\hline simulações & $\hat{\mu}_{x}$ & $\hat{\alpha}_{2}$ & $\hat{\alpha}_{3}$ & $\hat{\beta}_{2}$ & $\hat{\beta}_{3}$ & $\hat{\sigma}_{x}^{2}$ & $\hat{\sigma}_{1}^{2}$ & $\hat{\sigma}_{2}^{2}$ & $\hat{\sigma}_{3}^{2}$ \\
\hline 100 & 1.563 & 6.334 & 6.673 & 0.123 & 0.131 & 27.642 & 8.197 & 8.028 & 7.883 \\
500 & 1.575 & 5.905 & 6.393 & 0.116 & 0.126 & 25.979 & 8.074 & 7.768 & 7.612 \\
1000 & 1.572 & 6.048 & 6.303 & 0.118 & 0.125 & 25.809 & 7.748 & 7.805 & 7.721 \\
2000 & 1.555 & 5.982 & 6.170 & 0.117 & 0.122 & 25.697 & 7.710 & 7.835 & 8.003 \\
3000 & 1.558 & 5.899 & 6.153 & 0.116 & 0.122 & 25.660 & 7.842 & 7.819 & 7.906 \\
4000 & 1.559 & 5.898 & 6.108 & 0.116 & 0.121 & 25.593 & 7.845 & 7.806 & 7.907 \\
5000 & 1.553 & 5.988 & 6.125 & 0.118 & 0.121 & 25.410 & 7.902 & 7.817 & 7.857 \\
\hline
\end{tabular}




\section{Capítulo 3}

\section{Aplicações}

\subsection{Introdução}

Neste capítulo desenvolvemos um estudo detalhado para os dados das árvores apresentados no capítulo 1, considerando apenas o primeiro observador e a primeira observação para todos os instrumentos e todas as árvores, utilizando os seguintes procedimentos: análise de variância, máxima verossimilhança utilizando os algoritmos BFGS e EM, avaliando também a precisão e eficiência dos instrumentos. Para finalizar, para ambas as hipóteses de interesse foram implementados testes da razão de verossimilhança utilizando as estimativas obtidas pelo BFGS e de Wald utilizando as estimativas obtidas pelo EM e calculando também a matriz de informação.

\subsection{Hipóteses de Interesse}

Comparar instrumentos de medição significa fazer inferências sobre os parâmetros que medem os vícios aditivos e multiplicativos conjuntamente ou separadamente, dependendo do interesse. Nesse caso estamos interessados em testar as hipóteses descritas no capítulo 2.

Escrevendo as hipóteses $H_{01}$ e $H_{02}$ na forma $H_{0}: A \theta=\mathbf{b}$, vamos utilizar a estatística de Wald mencionada na seção anterior para efetuar os testes.

\subsection{Análise de Variância}

Primeiramente consideremos um modelo de análise de variância com um fator (Neter, 1996), para os dados das árvores sendo que, neste caso, a hipótese nula não foi rejeitada (p-valor maior que 0,05). Esta hipótese não foi rejeitada também para dados simulados, de modo que, esse procedimento não pareceu ser adequado para comparação de instrumentos de medição, conforme pode ser observado na saída do Minitab logo abaixo:

Figura 3.1 - saída do MINITAB versão 13 da análise de variância. 


\begin{tabular}{|c|c|c|c|c|}
\hline \multicolumn{5}{|c|}{ Analysis of Variance for altura } \\
\hline Source & $\mathrm{DF}$ & SS & MS & $\mathrm{P}$ \\
\hline instrume & 4 & 8.28 & 2.07 & 0.931 \\
\hline Error & 45 & 442.26 & 9.83 & \\
\hline \multirow[t]{2}{*}{ Total } & 49 & 450.54 & & \\
\hline & & & & $\begin{array}{l}\text { Individual } 95 \% \text { CIs For Mean } \\
\text { Based on Pooled StDev }\end{array}$ \\
\hline Level & $\mathrm{N}$ & Mean & StDev & ------+----------+----------+ \\
\hline 1 & 10 & 19.360 & 3.156 & $(------------*------------)$ \\
\hline 2 & 10 & 20.310 & 3.265 & $(------------*-------------)$ \\
\hline 3 & 10 & 19.350 & 3.241 & $(------------*------------)$ \\
\hline 4 & 10 & 19.300 & 3.068 & $(------------*-----------)$ \\
\hline 5 & 10 & 19.200 & 2.932 & $(------------*-----------)$ \\
\hline Pooled St & ev $=$ & 3.135 & & 21.0 \\
\hline
\end{tabular}

Como a análise de variância utilizada em Simões et al, (1963) não consegue nesse caso atender aos nossos objetivos principais, uma vez que apenas compara médias e não têm condições de comparar viéses e precisões, partimos para outros testes conforme pode ser verificado na seção seguinte.

\subsection{Estimadores de máxima verossimilhança}

Numa primeira etapa de estudo apresentada a seguir foram calculados os testes de Wald e teste da razão de verossimilhança utilizando 3 instrumentos de cada vez, em uma segunda etapa utilizando 4 instrumentos de cada vez e numa ultima etapa utilizando os cinco instrumentos para um mesmo observador. Para facilitar na construção da tabela eles foram numerados da seguinte maneira: $1=$ trena (instrumento padrão), 2 $=$ Blume-Leiss, $3=$ Haga, $4=$ Weiss e $5=$ Prancheta dendrométrica.

Ocorre que, para a implementação do teste, necessitamos dos estimadores de máxima verossimilhança, que foram calculados utilizando o programa Ox e o algorítmo EM, obtendo assim os estimadores da tabela 3.1 abaixo.

Como era de esperar, neste caso os estimadores obtidos pelos dois métodos coincidem, e é claro também com os estimadores explícitos obtidos no capítulo 2.

Entretanto, notamos problemas de convergência do Ox, especialmente em situações onde o tamanho da amostra é pequeno. 
Tabelas 3.1 - Estimativas dos parâmetros para combinações de três instrumentos para os dados das árvores.

\begin{tabular}{|c|c|c|c|c|c|c|c|c|c|}
\hline instrumentos & $\hat{\mu}_{x}$ & $\hat{\alpha}_{2}$ & $\hat{\alpha}_{3}$ & $\hat{\beta}_{2}$ & $\hat{\beta}_{3}$ & $\hat{\sigma}_{x}^{2}$ & $\hat{\sigma}_{1}^{2}$ & $\hat{\sigma}_{2}^{2}$ & $\hat{\sigma}_{3}^{2}$ \\
\hline 2 e 3 & 19.360 & 0.490 & -0.072 & 1.024 & 1.003 & 8.921 & 0.046 & 0.246 & 0.475 \\
2 e 4 & 19.360 & 19.300 & 18.374 & 0.052 & 0.048 & 3527.4 & 3185.4 & -0.010 & 0.410 \\
2 e 5 & 19.360 & 0.414 & 1.719 & 1.028 & 0.903 & 8.887 & 0.080 & 0.210 & 0.493 \\
3 e 4 & 19.360 & -0.973 & 0.360 & 1.050 & 0.978 & 8.523 & 0.444 & 0.056 & 0.317 \\
3 e 5 & 19.360 & 0.084 & 1.926 & 0.995 & 0.892 & 8.993 & -0.026 & 0.547 & 0.578 \\
4 e 5 & 19.360 & 0.741 & 1.340 & 0.959 & 0.923 & 8.698 & 0.269 & 0.481 & 0.336 \\
\hline
\end{tabular}

Tabelas 3.2 - Estimativas dos parâmetros para combinações de quatro instrumentos.

\begin{tabular}{|c|c|c|c|c|c|c|c|c|c|c|c|c|}
\hline instrumentos & $\hat{\mu}_{x}$ & $\hat{\alpha}_{2}$ & $\hat{\alpha}_{3}$ & $\hat{\alpha}_{4}$ & $\hat{\beta}_{2}$ & $\hat{\beta}_{3}$ & $\hat{\beta}_{4}$ & $\hat{\sigma}_{x}^{2}$ & $\hat{\sigma}_{1}^{2}$ & $\hat{\sigma}_{2}^{2}$ & $\hat{\sigma}_{3}^{2}$ & $\hat{\sigma}_{4}^{2}$ \\
\hline 2,3 e 4 & 19.360 & 0.206 & -0.351 & 0.613 & 1.038 & 1.018 & 0.965 & 8.758 & 0.208 & 0.150 & 0.384 & 0.314 \\
2,3 e 5 & 19.360 & 0.482 & -0.064 & 1.784 & 1.024 & 1.003 & 0.900 & 8.919 & 0.047 & 0.239 & 0.483 & 0.520 \\
2,4 e 5 & 19.360 & -11.048 & -9.458 & -7.743 & 1.620 & 1.485 & 1.392 & 3.658 & 1.346 & -0.002 & 0.403 & 0.654 \\
3,4 e 5 & 19.360 & -0.669 & 0.426 & 1.606 & 1.034 & 0.975 & 0.909 & 8.642 & 0.672 & 0.331 & 0.0708 & 0.083 \\
\hline
\end{tabular}

Para $p=3$ os três métodos podem ser utilizados (momentos, EM e BFGS do Ox), para a obtenção dos estimadores de máximo verossimilhança.

Em termos de convergência, é possível observar o seguinte:

- Nas combinações de quatro instrumentos(ver tabela 3.2) foi possível verificar que a convergência foi obtida em uma quantidade de iterações entre 50 a 100.

- No caso em que foram utilizados os cinco instrumentos(tabela 3.8), a quantidade de iterações foi por volta de 300.

- Nos casos em que foram utilizados combinações de 3 instrumentos (que não consta na tabela 3,1, a quantidade de iterações foi por volta de 50.

\subsection{Eficiência dos instrumentos}

Para os dados das árvores obtemos os seguintes valores para eficiência para cada grupo de três instrumentos: 
Tabela 3.3 - Resultados de eficiência para combinações de três instrumentos.

\begin{tabular}{c|c|c|c}
\hline instrumentos & \multicolumn{3}{|c}{ eficiência } \\
\hline 2 e 3 & $\mathrm{e}_{1}=0.99489$ & $\mathrm{e}_{2}=0.97440$ & $\mathrm{e}_{3}=0.94975$ \\
2 e 4 & $\mathrm{e}_{1}=0.52543$ & $\mathrm{e}_{2}=1.0000$ & $\mathrm{e}_{4}=0.95167$ \\
2 e 5 & $\mathrm{e}_{1}=0.99109$ & $\mathrm{e}_{2}=0.97813$ & $\mathrm{e}_{5}=0.93631$ \\
3 e 4 & $\mathrm{e}_{1}=0.95052$ & $\mathrm{e}_{3}=0.99408$ & $\mathrm{e}_{4}=0.96262$ \\
3 e 5 & $\mathrm{e}_{1}=1.0029$ & $\mathrm{e}_{3}=0.94213$ & $\mathrm{e}_{5}=0.92526$ \\
4 e 5 & $\mathrm{e}_{1}=0.97005$ & $\mathrm{e}_{4}=0.94324$ & $\mathrm{e}_{5}=0.95662$ \\
\hline
\end{tabular}

Para os dados das árvores obtemos os seguintes valores para precisão para cada grupo de tres instrumentos:

Tabela 3.4 - Precisão para combinações de três instrumentos.

\begin{tabular}{c|c|c|c}
\hline instrumentos & \multicolumn{3}{|c}{ precisão } \\
\hline 2 e 3 & $\pi_{1}=21.822$ & $\pi_{2}=4.2665$ & $\pi_{3}=2.1198$ \\
2 e 4 & $\pi_{1}=0.00031388$ & $\pi_{2}=0.27376$ & $\pi_{4}=0.005589$ \\
2 e 5 & $\pi_{1}=12.521$ & $\pi_{2}=5.0330$ & $\pi_{5}=1.6544$ \\
3 e 4 & $\pi_{1}=2.2541$ & $\pi_{3}=19.708$ & $\pi_{4}=3.0216$ \\
3 e 5 & $\pi_{1}=38.011$ & $\pi_{3}=1.8104$ & $\pi_{5}=1.3766$ \\
4 e 5 & $\pi_{1}=3.7242$ & $\pi_{4}=1.9106$ & $\pi_{5}=2.5354$ \\
\hline
\end{tabular}

É possível verificar que o primeiro instrumento, embora sendo considerado como sem viés, em geral foi considerado como o menos eficiente e de maior precisão.

Para os dados das árvores obtemos os seguintes valores para eficiência para cada grupo de quatro instrumentos: 
Tabela 3.5 - Valores de eficiência para combinações de quatro instrumentos.

\begin{tabular}{c|c|c|c|c}
\hline instrumentos & \multicolumn{4}{|c}{ eficiência } \\
\hline 2,3 e 4 & $\mathrm{e}_{1}=0.97678$ & $\mathrm{e}_{2}=0.98434$ & $\mathrm{e}_{3}=0.95943$ & $\mathrm{e}_{4}=0.96297$ \\
2,3 e 5 & $\mathrm{e}_{1}=0.99478$ & $\mathrm{e}_{2}=0.97506$ & $\mathrm{e}_{3}=0.94888$ & $\mathrm{e}_{5}=0.93281$ \\
2,4 e 5 & $\mathrm{e}_{1}=0.73094$ & $\mathrm{e}_{2}=1.000$ & $\mathrm{e}_{4}=0.95243$ & $\mathrm{e}_{5}=0.91553$ \\
3,4 e 5 & $\mathrm{e}_{1}=0.96376$ & $\mathrm{e}_{3}=0.97747$ & $\mathrm{e}_{4}=0.96922$ & $\mathrm{e}_{5}=0.92236$ \\
\hline
\end{tabular}

Para os dados das árvores obtemos os seguintes valores para precisão para cada grupo de quatro instrumentos:

Tabela 3.6 - Valores de precisão para combinações de quatro instrumentos.

\begin{tabular}{c|c|c|c|c}
\hline instrumentos & \multicolumn{4}{|c}{ precisão } \\
\hline 2,3 e 4 & $\pi_{1}=4.8024$ & $\pi_{2}=7.1752$ & $\pi_{3}=2.7001$ & $\pi_{4}=2.9694$ \\
2,3 e 5 & $\pi_{1}=21.213$ & $\pi_{2}=4.3831$ & $\pi_{3}=2.0810$ & $\pi_{5}=1.5567$ \\
2,4 e 5 & $\pi_{1}=0.74270$ & $\pi_{2}=1542.4$ & $\pi_{4}=5.4734$ & $\pi_{5}=2.9631$ \\
3,4 e 5 & $\pi_{1}=3.0776$ & $\pi_{3}=5.0202$ & $\pi_{4}=3.6436$ & $\pi_{5}=1.3747$ \\
\hline
\end{tabular}

Agora, considerando todos os instrumentos, obtemos os seguintes resultados para eficiência e precisão:

Tabela 3.7 - Valores para estimativa e precisão considerando todos os instrumentos.

\begin{tabular}{|c|c|c|c|c|c|}
\hline instrumentos & $\mathrm{e}_{1}$ & $\mathrm{e}_{2}$ & $\mathrm{e}_{3}$ & $\mathrm{e}_{4}$ & $\mathrm{e}_{5}$ \\
$2,3,4$ e 5 & 0.97974 & 0.98422 & 0.95643 & 0.96127 & 0.93422 \\
\hline instrumentos & $\pi_{1}$ & $\pi_{2}$ & $\pi_{3}$ & $\pi_{4}$ & $\pi_{5}$ \\
$2,3,4$ e 5 & 5.5044 & 7.1013 & 2.4989 & 2.8254 & 1.6166 \\
\hline
\end{tabular}

É possível verificar que o quinto instrumento foi considerado como o menos eficiente é o de menor precisão.

O segundo instrumento foi considerado o mais eficiente e o mais preciso.

\subsection{Teste de Wald}

Os estimadores da matriz de informação de Fisher (veja apêndice F), $\hat{I}$ e suas inversas, $\hat{I}^{-1}$ são dadas a seguir. Temos:

\section{Instrumentos 2 e 3:}

$$
\hat{I}=
$$




$\begin{array}{ccclll}1.1166 & 0.16497 & 0.083603 & 3.1938 & 1.6186 & 0.00000 \\ 0.16497 & 34.576 & -3.1081 & 669.39 & -60.174 & 0.00000 \\ 0.083603 & -3.1081 & 19.478 & -60.174 & 377.10 & 0.00000 \\ 3.1938 & 669.39 & -60.174 & 13267 . & -1192.5 & 0.16432 \\ 1.6186 & -60.174 & 377.10 & -1192.5 & 7473.7 & 0.083272 \\ 0.00000 & 0.00000 & 0.00000 & 0.16432 & 0.083272 & 0.062336 \\ 0.00000 & 0.00000 & 0.00000 & -24.746 & -12.541 & 0.037308 \\ 0.00000 & 0.00000 & 0.00000 & 5.0883 & -0.45740 & 0.039101 \\ 0.00000 & 0.00000 & 0.00000 & -0.23180 & 1.4527 & 0.037546 \\ & & & & & \\ 0.00000 & 0.00000 & 0.00000 & \\ 0.00000 & 0.00000 & 0.00000 & \\ 0.00000 & 0.00000 & 0.00000 & \\ -24.746 & 5.0883 & -0.23180 & \\ -12.541 & -0.45740 & 1.4527 & \\ 0.037308 & 0.039101 & 0.037546 & \\ 125.34 & 51.566 & 13.243 & \\ 51.566 & 59.775 & 0.48303 & \\ 13.243 & 0.48303 & 18.970 & \\ \hat{I}^{-1}= & & & & \end{array}$

$\begin{array}{rrrrrr}0.89664 & -0.0046913 & -0.0045971 & 2.7256 \mathrm{e}-015 & -4.2543 \mathrm{e}-016 & -1.9869 \mathrm{e}-015 \\ -0.0046913 & 1.3278 & 0.25524 & -0.067069 & -0.012941 & 0.18071 \\ -0.0045971 & 0.25524 & 2.2984 & -0.012941 & -0.11603 & 0.17495 \\ -7.2456 \mathrm{e}-022 & -0.067069 & -0.012941 & 0.0034643 & 0.00066843 & -0.0093341 \\ 4.4983 \mathrm{e}-019 & -0.012941 & -0.11603 & 0.00066843 & 0.0059932 & -0.0090365 \\ -5.5324 \mathrm{e}-019 & 0.18071 & 0.17495 & -0.0093341 & -0.0090365 & 16.103 \\ 8.1382 \mathrm{e}-020 & -0.029202 & -0.026247 & 0.0015084 & 0.0013557 & -0.00056858 \\ -6.5624 \mathrm{e}-020 & 0.030526 & 0.022531 & -0.0015768 & -0.0011638 & -0.0090701 \\ -8.8504 \mathrm{e}-020 & 0.019423 & 0.026131 & -0.0010033 & -0.0013497 & -0.030667\end{array}$

$\begin{array}{rrr}-2.0931 \mathrm{e}-016 & -4.8064 \mathrm{e}-016 & -2.0343 \mathrm{e}-016 \\ -0.029202 & 0.030526 & 0.019423 \\ -0.026247 & 0.022531 & 0.026131 \\ 0.0015084 & -0.0015768 & -0.0010033 \\ 0.0013557 & -0.0011638 & -0.0013497 \\ -0.00056858 & -0.0090701 & -0.030667 \\ 0.014706 & -0.012723 & -0.010027 \\ -0.012723 & 0.027770 & 0.0082630 \\ -0.010027 & 0.0082630 & 0.059656\end{array}$


Instrumentos 2 e 4 :

$\hat{I}=$

$\begin{array}{rrr}0.0028380 & 0.055583 & -0.0012368 \\ 0.055583 & 23.217 & -22.888 \\ -0.0012368 & -22.888 & 24.924 \\ 1.0761 & 449.48 & -443.12 \\ -0.023945 & -443.12 & 482.53 \\ 0.00000 & 0.00000 & 0.00000 \\ 0.00000 & 0.00000 & 0.00000 \\ 0.00000 & 0.00000 & 0.00000 \\ 0.00000 & 0.00000 & 0.00000 \\ & & \\ 0.00000 & 0.00000 & 0.00000 \\ 0.00000 & 0.00000 & 0.00000 \\ 0.00000 & 0.00000 & 0.00000 \\ 7.2189 \mathrm{e}-005 & 455.20 & 9.9855 \\ -1.6063 \mathrm{e}-006 & -448.75 & -10.874 \\ 5.5295 \mathrm{e}-013 & 1.5057 \mathrm{e}-015 & 1.2642 \mathrm{e}-015 \\ 4.9377 \mathrm{e}-007 & 0.00018936 & 9.3761 \mathrm{e}-008 \\ 0.00018936 & 26.952 & 26.194 \\ 9.3761 \mathrm{e}-008 & 26.194 & 31.061\end{array}$

$\hat{I}^{-1}=$

\begin{tabular}{rrrrrr}
671.33 & -16.625 & -15.233 & $-1.1739 \mathrm{e}-014$ & $-1.0187 \mathrm{e}-014$ & $1.6012 \mathrm{e}-009$ \\
-16.625 & 0.97854 & 0.88829 & -0.0057863 & -0.0048236 & 784.67 \\
-15.233 & 0.88829 & 0.85505 & -0.0048236 & -0.0044272 & 652.87 \\
$-9.4414 \mathrm{e}-015$ & -0.0057863 & -0.0048236 & 0.00029888 & 0.00024915 & -40.531 \\
$-7.8904 \mathrm{e}-015$ & -0.0048236 & -0.0044272 & 0.00024915 & 0.00022868 & -33.723 \\
$1.2803 \mathrm{e}-009$ & 784.67 & 652.87 & -40.531 & -33.723 & $7.9796 \mathrm{e}+006$ \\
$-5.8624 \mathrm{e}-011$ & -36.357 & -16.022 & 1.8780 & 0.82760 & $-2.5694 \mathrm{e}+005$ \\
$1.5646 \mathrm{e}-013$ & 0.097006 & 0.043596 & -0.0050106 & -0.0022519 & 685.40 \\
$-1.3167 \mathrm{e}-013$ & -0.081633 & -0.036764 & 0.0042166 & 0.0018990 & -576.77 \\
\multicolumn{7}{c}{$1.7861 \mathrm{e}-013$} & $-1.5468 \mathrm{e}-013$ & & \\
$-7.0566 \mathrm{e}-011$ & 1.013 & & \\
-36.357 & 0.097006 & -0.081633 & & \\
-16.022 & 0.043596 & -0.036764 & & \\
1.8780 & -0.0050106 & 0.0042166 & & \\
0.82760 & -0.0022519 & 0.0018990 & &
\end{tabular}




$\begin{array}{rrr}2.0937 e+006 & -179.22 & 150.81 \\ -179.22 & 0.46940 & -0.39502 \\ 150.81 & -0.39502 & 0.36462\end{array}$

Instrumentos 2 e 5 :

$\hat{I}=$

$\begin{array}{rrrrrr}1.1187 & 0.28524 & 0.10671 & 5.5222 & 2.0659 & 0.00000 \\ 0.28524 & 35.243 & -4.6442 & 682.30 & -89.912 & 0.00000 \\ 0.10671 & -4.6442 & 18.554 & -89.912 & 359.21 & 0.00000 \\ 5.5222 & 682.30 & -89.912 & 13521 . & -1781.5 & 0.28358 \\ 2.0659 & -89.912 & 359.21 & -1781.5 & 7118.2 & 0.10609 \\ 0.00000 & 0.00000 & 0.00000 & 0.28358 & 0.10609 & 0.062579 \\ 0.00000 & 0.00000 & 0.00000 & -20.569 & -7.6949 & 0.026591 \\ 0.00000 & 0.00000 & 0.00000 & 8.9332 & -1.1772 & 0.028083 \\ 0.00000 & 0.00000 & 0.00000 & -0.44040 & 1.7594 & 0.021680\end{array}$

$\begin{array}{rrr}0.00000 & 0.00000 & 0.00000 \\ 0.00000 & 0.00000 & 0.00000 \\ 0.00000 & 0.00000 & 0.00000 \\ -20.569 & 8.9332 & -0.44040 \\ -7.6949 & -1.1772 & 1.7594 \\ 0.026591 & 0.028083 & 0.021680 \\ 97.097 & 50.368 & 7.0493 \\ 50.368 & 62.102 & 1.0784 \\ 7.0493 & 1.0784 & 17.213\end{array}$

$\hat{I}^{-1}=$

$\begin{array}{rrrrrr}0.89664 & -0.0082073 & -0.0072112 & 6.5234 \mathrm{e}-016 & 2.5548 \mathrm{e}-018 & -8.1826 \mathrm{e}-015 \\ -0.0082073 & 1.3537 & 0.37217 & -0.068405 & -0.018841 & 0.33573 \\ -0.0072112 & 0.37217 & 2.4610 & -0.018841 & -0.12424 & 0.29027 \\ -1.8498 \mathrm{e}-018 & -0.068405 & -0.018841 & 0.0035333 & 0.00097318 & -0.017342 \\ 1.6871 \mathrm{e}-018 & -0.018841 & -0.12424 & 0.00097318 & 0.0064172 & -0.014993 \\ 5.4294 \mathrm{e}-018 & 0.33573 & 0.29027 & -0.017342 & -0.014993 & 16.092 \\ -7.1415 \mathrm{e}-019 & -0.037493 & -0.026573 & 0.0019366 & 0.0013725 & -0.0097760 \\ 8.7464 \mathrm{e}-019 & 0.039521 & 0.021404 & -0.0020414 & -0.0011056 & 0.0031294 \\ 1.1052 \mathrm{e}-020 & 0.012632 & 0.021393 & -0.00065247 & -0.0011050 & -0.015371\end{array}$

$2.2269 \mathrm{e}-016-1.9474 \mathrm{e}-016-2.8790 \mathrm{e}-016$

$\begin{array}{lll}-0.037493 & 0.039521 & 0.012632\end{array}$

$\begin{array}{lll}-0.026573 & 0.021404 & 0.021393\end{array}$ 


$\begin{array}{rrr}0.0019366 & -0.0020414 & -0.00065247 \\ 0.0013725 & -0.0011056 & -0.0011050 \\ -0.0097760 & 0.0031294 & -0.015371 \\ 0.019685 & -0.016090 & -0.0071320 \\ -0.016090 & 0.029340 & 0.0048079 \\ -0.0071320 & 0.0048079 & 0.060832\end{array}$

\section{Instrumentos 3 e 4:}

$\hat{I}=$

$\begin{array}{rrrrrr}1.1678 & 0.87736 & 0.14437 & 16.986 & 2.7951 & 0.00000 \\ 0.87736 & 38.406 & -23.095 & 743.54 & -447.12 & 0.00000 \\ 0.14437 & -23.095 & 27.770 & -447.12 & 537.62 & 0.00000 \\ 16.986 & 743.54 & -447.12 & 14726 . & -8851.2 & 0.87326 \\ 2.7951 & -447.12 & 537.62 & -8851.2 & 10644 . & 0.14370 \\ 0.00000 & 0.00000 & 0.00000 & 0.87326 & 0.14370 & 0.068193 \\ 0.00000 & 0.00000 & 0.00000 & -1.5136 & -0.24906 & 0.00055509 \\ 0.00000 & 0.00000 & 0.00000 & 28.718 & -17.269 & 0.00061200 \\ 0.00000 & 0.00000 & 0.00000 & -2.8417 & 3.4169 & 0.00053128\end{array}$

$\begin{array}{rrr}0.00000 & 0.00000 & 0.00000 \\ 0.00000 & 0.00000 & 0.00000 \\ 0.00000 & 0.00000 & 0.00000 \\ -1.5136 & 28.718 & -2.8417 \\ -0.24906 & -17.269 & 3.4169 \\ 0.00055509 & 0.00061200 & 0.00053128 \\ 21.047 & 14.204 & 0.38463 \\ 14.204 & 73.750 & 26.669 \\ 0.38463 & 26.669 & 38.558\end{array}$

$\hat{I}^{-1}=$

$\begin{array}{rrrrrr}0.89664 & -0.046583 & -0.043402 & 3.7009 \mathrm{e}-016 & -9.1859 \mathrm{e}-018 & 2.3309 \mathrm{e}-015 \\ -0.046583 & 2.5508 & 2.0614 & -0.12894 & -0.10412 & 1.8707 \\ -0.043402 & 2.0614 & 3.3538 & -0.10412 & -0.16941 & 1.6904 \\ 5.4690 \mathrm{e}-017 & -0.12894 & -0.10412 & 0.0066601 & 0.0053783 & -0.096628 \\ 4.5147 \mathrm{e}-017 & -0.10412 & -0.16941 & 0.0053783 & 0.0087503 & -0.087312 \\ -7.9555 \mathrm{e}-016 & 1.8707 & 1.6904 & -0.096628 & -0.087312 & 16.086 \\ 1.6768 \mathrm{e}-017 & -0.040148 & -0.0097216 & 0.0020738 & 0.00050215 & -0.028027 \\ -1.8538 \mathrm{e}-017 & 0.044684 & 7.3772 \mathrm{e}-005 & -0.0023081 & -3.8105 \mathrm{e}-006 & 0.029608 \\ 1.2695 \mathrm{e}-017 & -0.030806 & 0.0073612 & 0.0015912 & -0.00038023 & -0.019804\end{array}$




$$
\begin{array}{rrr}
-1.6451 \mathrm{e}-016 & 1.4859 \mathrm{e}-016 & -1.2725 \mathrm{e}-016 \\
-0.040148 & 0.044684 & -0.030806 \\
-0.0097216 & 7.3772 \mathrm{e}-005 & 0.0073612 \\
0.0020738 & -0.0023081 & 0.0015912 \\
0.00050215 & -3.8105 \mathrm{e}-006 & -0.00038023 \\
-0.028027 & 0.029608 & -0.019804 \\
0.058003 & -0.015591 & 0.010313 \\
-0.015591 & 0.023290 & -0.016123 \\
0.010313 & -0.016123 & 0.037135
\end{array}
$$

\section{Instrumentos 3 e 5:}

$$
\begin{array}{rrrrrr}
\hat{I}= \\
\\
1.1156 & -0.058280 & -0.049423 & -1.1283 & -0.95682 & 0.00000 \\
-0.058280 & 19.235 & 0.80857 & 372.39 & 15.654 & 0.00000 \\
-0.049423 & 0.80857 & 17.976 & 15.654 & 348.02 & 0.00000 \\
-1.1283 & 372.39 & 15.654 & 7383.1 & 310.38 & -0.058467 \\
-0.95682 & 15.654 & 348.02 & 310.38 & 6899.8 & -0.04958 \\
0.00000 & 0.00000 & 0.00000 & -0.058467 & -0.049581 & 0.062225 \\
0.00000 & 0.00000 & 0.00000 & -21.814 & -18.499 & 0.074136 \\
0.00000 & 0.00000 & 0.00000 & -1.0081 & -0.042377 & 0.073417 \\
0.00000 & 0.00000 & 0.00000 & -0.035936 & -0.79893 & 0.059024
\end{array}
$$$$
372.39
$$$$
15.654
$$$$
0.00000
$$$$
348.02
$$$$
0.00000
$$$$
310.38
$$$$
-0.058467
$$$$
6899.8
$$$$
-0.049581
$$

$-0.058467$

$-0.049581$

0.062225

$-21.814$

$-18.499$

0.074136

$-1.0081$

$-0.042377$

0.073417

$-0.035936$

$-0.79893$

0.059024

$\begin{array}{rrr}0.00000 & 0.00000 & 0.00000 \\ 0.00000 & 0.00000 & 0.00000 \\ 0.00000 & 0.00000 & 0.00000 \\ -21.814 & -1.0081 & -0.035936 \\ -18.499 & -0.042377 & -0.79893 \\ 0.074136 & 0.073417 & 0.059024 \\ 65.219 & 19.843 & 14.270 \\ 19.843 & 18.500 & 0.032689 \\ 14.270 & 0.032689 & 16.157\end{array}$

$\hat{I}^{-1}=$

$\begin{array}{rrrrrr}0.89664 & 0.0026180 & 0.0023474 & 1.0177 e-015 & 9.1823 e-017 & -1.3905 e-015 \\ 0.0026180 & 2.4008 & 0.067317 & -0.12132 & -0.0035978 & -0.18494 \\ 0.0023474 & 0.067317 & 2.5234 & -0.0035978 & -0.12746 & -0.16762 \\ -5.7018 \mathrm{e}-020 & -0.12132 & -0.0035978 & 0.0062665 & 0.00018584 & 0.0095525 \\ 2.4469 \mathrm{e}-019 & -0.0035978 & -0.12746 & 0.00018584 & 0.0065838 & 0.0086582\end{array}$




$\begin{array}{rrrr}2.2676 \mathrm{e}-019 & -0.18494 & -0.16762 & 0.009 \\ 1.0116 \mathrm{e}-019 & -0.082373 & -0.074741 & 0.004 \\ -1.1181 \mathrm{e}-019 & 0.082342 & 0.080240 & -0.004 \\ -7.7970 \mathrm{e}-020 & 0.072814 & 0.060151 & -0.003 \\ & & & \\ 1.4784 \mathrm{e}-015 & 1.1224 \mathrm{e}-015 & 2.5520 \mathrm{e}-015 \\ -0.082373 & 0.082342 & 0.072814 \\ -0.074741 & 0.080240 & 0.060151 \\ 0.0042548 & -0.0042532 & -0.0037610 \\ 0.0038606 & -0.0041446 & -0.0031070 \\ 0.040450 & -0.10719 & -0.094439 \\ 0.036916 & -0.039459 & -0.032472 \\ -0.039459 & 0.096503 & 0.034832 \\ -0.032472 & 0.034832 & 0.090685\end{array}$

\section{Instrumentos 4 e 5:}

$\hat{I}=$

$\begin{array}{rrrrrr}1.1338 & 0.27657 & 0.38138 & 5.3544 & 7.3835 & 0.00000 \\ 0.27657 & 15.996 & -6.6112 & 309.68 & -127.99 & 0.00000 \\ 0.38138 & -6.6112 & 20.674 & -127.99 & 400.26 & 0.00000 \\ 5.3544 & 309.68 & -127.99 & 6133.3 & -2533.8 & 0.27273 \\ 7.3835 & -127.99 & 400.26 & -2533.8 & 7927.4 & 0.37608 \\ 0.00000 & 0.00000 & 0.00000 & 0.27273 & 0.37608 & 0.064270 \\ 0.00000 & 0.00000 & 0.00000 & -4.0270 & -5.5530 & 0.013354 \\ 0.00000 & 0.00000 & 0.00000 & 3.8479 & -1.5904 & 0.012272 \\ 0.00000 & 0.00000 & 0.00000 & -2.1930 & 6.8581 & 0.011365\end{array}$

$\begin{array}{rrr}0.00000 & 0.00000 & 0.00000 \\ 0.00000 & 0.00000 & 0.00000 \\ 0.00000 & 0.00000 & 0.00000 \\ -4.0270 & 3.8479 & -2.1930 \\ -5.5530 & -1.5904 & 6.8581 \\ 0.013354 & 0.012272 & 0.011365 \\ 21.015 & 4.0129 & 7.6306 \\ 4.0129 & 12.794 & 2.1854 \\ 7.6306 & 2.1854 & 21.372\end{array}$

$\hat{I}^{-1}=$

$0.89664-0.025741-0.024771 \quad 3.3705 e-016 \quad 2.2531 e-016-1.6819 e-016$ 


$\begin{array}{rrrrrr}-0.025741 & 3.3498 & 1.1365 & -0.16927 & -0.057477 & 1.0537 \\ -0.024771 & 1.1365 & 2.6332 & -0.057477 & -0.13310 & 1.0246 \\ -2.2455 \mathrm{e}-017 & -0.16927 & -0.057477 & 0.0087431 & 0.0029689 & -0.054426 \\ -1.1603 \mathrm{e}-017 & -0.057477 & -0.13310 & 0.0029689 & 0.0068750 & -0.052923 \\ 1.6314 \mathrm{e}-016 & 1.0537 & 1.0246 & -0.054426 & -0.052923 & 16.105 \\ -1.0505 \mathrm{e}-017 & -0.066392 & -0.071012 & 0.0034294 & 0.0036679 & -0.041785 \\ 7.7167 \mathrm{e}-018 & 0.060499 & 0.011711 & -0.0031249 & -0.00060492 & 0.0044952 \\ 4.2941 \mathrm{e}-018 & 0.018033 & 0.060425 & -0.00093148 & -0.0031211 & 0.017293\end{array}$

$\begin{array}{rrr}3.1619 \mathrm{e}-016 & 3.8976 \mathrm{e}-017 & -1.6001 \mathrm{e}-016 \\ -0.066392 & 0.060499 & 0.018033 \\ -0.071012 & 0.011711 & 0.060425 \\ 0.0034294 & -0.0031249 & -0.00093148 \\ 0.0036679 & -0.00060492 & -0.0031211 \\ -0.041785 & 0.0044952 & 0.017293 \\ 0.059691 & -0.015756 & -0.020504 \\ -0.015756 & 0.084503 & -0.0031444 \\ -0.020504 & -0.0031444 & 0.055330\end{array}$

\section{Instrumentos 2 e 3 :}

No caso de testar a hipótese:

$$
H_{01}: \alpha_{2}=\alpha_{3}=0, \beta_{2}=\beta_{3}=1 ;
$$

que pode ser escrita como $H_{01}: A \theta=\mathbf{b}$, com a matriz $\mathrm{A}$ e vetor $\mathbf{b}$ dados por:

$$
A=\left(\begin{array}{ccccccccc}
0 & 1 & 0 & 0 & 0 & 0 & 0 & 0 & 0 \\
0 & 0 & 1 & 0 & 0 & 0 & 0 & 0 & 0 \\
0 & 0 & 0 & 1 & 0 & 0 & 0 & 0 & 0 \\
0 & 0 & 0 & 0 & 1 & 0 & 0 & 0 & 0
\end{array}\right) \text { e } \mathbf{b}=\left(\begin{array}{c}
0 \\
0 \\
1 \\
1
\end{array}\right)
$$

de modo que, temos para cada caso:

$$
A \hat{\theta}-\mathbf{b}=\left(\begin{array}{llll}
0.49028 & -0.071690 & 0.023746 & 0.0031865
\end{array}\right)^{\prime},
$$

com:

$$
\left[A \hat{I}_{F}^{-1}(\hat{\theta}) A^{\prime}\right]^{-1}=\left(\begin{array}{cccc}
34.552 & -3.1205 & 668.92 & -60.413 \\
-3.1205 & 19.472 & -60.413 & 376.98 \\
668.92 & -60.413 & 13245 . & -1202.5 \\
-60.413 & 376.98 & -1202.5 & 7468.8
\end{array}\right)
$$

que leva ao valor da estatística $\mathrm{W}=31.407$. 
Comparando $\mathrm{W}$ com o valor crítico de qui-quadrado a um nível de significância de $5 \%$ com 4 graus de liberdade, $\chi_{s=4}^{2}(0,05)=9.488$, temos que $31.407>9.4882$, então rejeitamos a hipótese nula o que significa que os instrumentos têm vícios aditivos e multiplicativos, ou seja, que pelo menos um instrumento tem viés aditivo não nulo ou pelo menos um deles tem vício multiplicativo diferente de um.

Agora, passemos a verificar se esses vícios aditivos e multiplicativos são todos iguais entre eles. Para este caso a hipótese de interesse é dada por:

$$
H_{02}: \alpha_{2}=\alpha_{3}=\alpha, \beta_{2}=\beta_{3}=\beta ;
$$

onde a matriz A é dada por:

$$
A=\left(\begin{array}{ccccccccc}
0 & 1 & -1 & 0 & 0 & 0 & 0 & 0 & 0 \\
0 & 0 & 0 & 1 & -1 & 0 & 0 & 0 & 0
\end{array}\right),
$$

de modo que, $A \theta-\mathbf{b}=0$, de onde obtemos:

$$
\begin{aligned}
\mathbf{b}=A \hat{\theta}=\left(\begin{array}{ll}
0.56197 & 0.020559
\end{array}\right)^{\prime} \mathrm{e} \\
\\
\quad\left[A \hat{I}_{F}^{-1}(\hat{\theta}) A^{\prime}\right]^{-1}=\left(\begin{array}{ll}
13.876 & 268.65 \\
268.65 & 5324.1
\end{array}\right) .
\end{aligned}
$$

Neste caso o valor da estatística $\mathrm{W}=12.841$, que indica rejeição de $\mathrm{H}_{0}$, isto é, existe diferencas entre os vícios.

\section{Instrumentos 2 e 4 :}

No caso de testar a hipótese:

$$
H_{01}: \alpha_{2}=\alpha_{3}=0, \beta_{2}=\beta_{3}=1 ;
$$

que pode ser escrita como $H_{01}: A \theta=\mathbf{b}$, com a matriz $\mathrm{A}$ e vetor $\mathbf{b}$ dados por:

$$
A=\left(\begin{array}{ccccccccc}
0 & 1 & 0 & 0 & 0 & 0 & 0 & 0 & 0 \\
0 & 0 & 1 & 0 & 0 & 0 & 0 & 0 & 0 \\
0 & 0 & 0 & 1 & 0 & 0 & 0 & 0 & 0 \\
0 & 0 & 0 & 0 & 1 & 0 & 0 & 0 & 0
\end{array}\right) \text { e } \mathbf{b}=\left(\begin{array}{c}
0 \\
0 \\
1 \\
1
\end{array}\right)
$$

de modo que, temos para cada caso:

$$
A \hat{\theta}-\mathbf{b}=\left(\begin{array}{llll}
19.300 & 18.374 & -0.94782 & -0.95219
\end{array}\right)^{\prime}
$$


com:

$$
\left[A \hat{I}_{F}^{-1}(\hat{\theta}) A^{\prime}\right]^{-1}=\left(\begin{array}{cccc}
22.129 & -22.864 & 428.41 & -442.65 \\
-22.864 & 24.924 & -442.65 & 482.52 \\
428.41 & -442.65 & 44765 . & -48306 . \\
-442.65 & 482.52 & -48306 . & 57009 .
\end{array}\right)
$$

que leva ao valor da estatística $\mathrm{W}=4280.4$.

Comparando $\mathrm{W}$ com o valor crítico de qui-quadrado a um nível de significância de $5 \%$ com 4 graus de liberdade, $\chi_{s=4}^{2}(0,05)=9.488$, temos que $4280.4>9.4882$, então rejeitamos a hipótese nula o que significa que os instrumentos têm vícios aditivos e multiplicativos, ou seja, que pelo menos um instrumento tem viés aditivo não nulo ou pelo menos um deles tem vício multiplicativo diferente de um.

Agora, passemos a verificar se esses vícios aditivos e multiplicativos são todos iguais entre eles. Para este caso a hipótese de interesse é dada por:

$$
H_{02}: \alpha_{2}=\alpha_{3}=\alpha, \beta_{2}=\beta_{3}=\beta
$$

onde a matriz A é dada por:

$$
A=\left(\begin{array}{ccccccccc}
0 & 1 & -1 & 0 & 0 & 0 & 0 & 0 & 0 \\
0 & 0 & 0 & 1 & -1 & 0 & 0 & 0 & 0
\end{array}\right),
$$

de modo que, $A \theta-\mathbf{b}=0$, de onde obtemos:

$$
\begin{gathered}
A \hat{\theta}=\left(\begin{array}{ll}
0.92545 & 0.0043672
\end{array}\right)^{\prime} \mathrm{e} \\
{\left[A \hat{I}_{F}^{-1}(\hat{\theta}) A^{\prime}\right]^{-1}=\left(\begin{array}{ll}
21.720 & 420.50 \\
420.50 & 42326 .
\end{array}\right) .}
\end{gathered}
$$

Neste caso o valor da estatística $W=22.809$, que indica a rejeição de $\mathrm{H}_{0}$, isto é, existe diferencas entre os vícios.

\section{Instrumentos 2 e 5:}

No caso de testar a hipótese:

$$
H_{01}: \alpha_{2}=\alpha_{3}=0, \beta_{2}=\beta_{3}=1 ;
$$

que pode ser escrita como $H_{01}: A \theta=\mathbf{b}$, com a matriz $\mathrm{A}$ e vetor $\mathbf{b}$ dados por: 


$$
A=\left(\begin{array}{ccccccccc}
0 & 1 & 0 & 0 & 0 & 0 & 0 & 0 & 0 \\
0 & 0 & 1 & 0 & 0 & 0 & 0 & 0 & 0 \\
0 & 0 & 0 & 1 & 0 & 0 & 0 & 0 & 0 \\
0 & 0 & 0 & 0 & 1 & 0 & 0 & 0 & 0
\end{array}\right) \text { e } \mathbf{b}=\left(\begin{array}{c}
0 \\
0 \\
1 \\
1
\end{array}\right)
$$

de modo que, temos para cada caso:

$$
A \hat{\theta}-\mathbf{b}=\left(\begin{array}{llll}
0.41437 & 1.7191 & 0.027667 & -0.097061
\end{array}\right)^{\prime}
$$

com:

$$
\begin{aligned}
& {\left[A \hat{I}_{F}^{-1}(\hat{\theta}) A^{\prime}\right]^{-1}=} \\
& \left(\begin{array}{cccc}
35.170 & -4.6714 & 680.89 & -90.439 \\
-4.6714 & 18.544 & -90.439 & 359.01 \\
680.89 & -90.439 & 13477 . & -1795.7 \\
-90.439 & 359.01 & -1795.7 & 7113.0
\end{array}\right)
\end{aligned}
$$

que leva ao valor da estatística $\mathrm{W}=35.634$.

Comparando $\mathrm{W}$ com o valor crítico de qui-quadrado a um nível de significância de $5 \%$ com 4 graus de liberdade, $\chi_{s=4}^{2}(0,05)=9.488$, temos que $35.634>9.4882$, então rejeitamos a hipótese nula o que significa que os instrumentos têm vícios aditivos e multiplicativos, ou seja, que pelo menos um instrumento tem viés aditivo não nulo ou pelo menos um deles tem vício multiplicativo diferente de um.

Agora, passemos a verificar se esses vícios aditivos e multiplicativos são todos iguais entre eles. Para este caso a hipótese de interesse é dada por:

$$
H_{02}: \alpha_{2}=\alpha_{3}=\alpha, \beta_{2}=\beta_{3}=\beta ;
$$

onde a matriz A é dada por:

$$
A=\left(\begin{array}{ccccccccc}
0 & 1 & -1 & 0 & 0 & 0 & 0 & 0 & 0 \\
0 & 0 & 0 & 1 & -1 & 0 & 0 & 0 & 0
\end{array}\right),
$$

de modo que, $A \theta-\mathbf{b}=0$, de onde obtemos:

$$
\begin{aligned}
& \mathbf{b}=A \hat{\theta}=\left(\begin{array}{ll}
-1.3047 & 0.12473
\end{array}\right)^{\prime} \mathrm{e} \\
& {\left[A \hat{I}_{F}^{-1}(\hat{\theta}) A^{\prime}\right]^{-1}=\left(\begin{array}{ll}
14.207 & 275.04 \\
275.04 & 5449.7
\end{array}\right) .}
\end{aligned}
$$


Neste caso o valor da estatística $\mathrm{W}=19.448$, que indica a rejeição de $\mathrm{H}_{0}$, isto é, existe diferencas entre os vícios.

\section{Instrumentos 3 e 4:}

No caso de testar a hipótese:

$$
H_{01}: \alpha_{2}=\alpha_{3}=0, \beta_{2}=\beta_{3}=1 ;
$$

que pode ser escrita como $H_{01}: A \theta=\mathbf{b}$, com a matriz $\mathrm{A}$ e vetor $\mathbf{b}$ dados por:

$$
A=\left(\begin{array}{ccccccccc}
0 & 1 & 0 & 0 & 0 & 0 & 0 & 0 & 0 \\
0 & 0 & 1 & 0 & 0 & 0 & 0 & 0 & 0 \\
0 & 0 & 0 & 1 & 0 & 0 & 0 & 0 & 0 \\
0 & 0 & 0 & 0 & 1 & 0 & 0 & 0 & 0
\end{array}\right) \text { e } \mathbf{b}=\left(\begin{array}{c}
0 \\
0 \\
1 \\
1
\end{array}\right)
$$

de modo que, temos para cada caso:

$$
A \hat{\theta}-\mathbf{b}=\left(\begin{array}{llll}
-0.97823 & 0.35969 & 0.050012 & -0.021678
\end{array}\right)^{\prime}
$$

com:

$$
\left[A \hat{I}_{F}^{-1}(\hat{\theta}) A^{\prime}\right]^{-1}=\left(\begin{array}{cccc}
37.747 & -23.203 & 730.77 & -449.22 \\
-23.203 & 27.752 & -449.22 & 537.28 \\
730.77 & -449.22 & 14446 . & -8880.1 \\
-449.22 & 537.28 & -8880.1 & 10629 .
\end{array}\right)
$$

que leva ao valor da estatística $\mathrm{W}=1.3254$.

Comparando $\mathrm{W}$ com o valor crítico da qui-quadrado a um nível de significância de $5 \%$ com 4 graus de liberdade, $\chi_{s=4}^{2}(0,05)=9.488$, temos que $1.3254<9.4882$, então não rejeitamos a hipótese nula o que significa que os instrumentos não têm vícios

aditivos e multiplicativos, ou seja, nenhum instrumento tem viés aditivo não nulo ou vício multiplicativo diferente de um.

Agora, passemos a verificar se esses vícios aditivos e multiplicativos são todos iguais entre eles. Para este caso a hipótese de interesse é dada por:

$$
H_{02}: \alpha_{2}=\alpha_{3}=\alpha, \beta_{2}=\beta_{3}=\beta ;
$$

onde a matriz A é dada por:

$$
A=\left(\begin{array}{ccccccccc}
0 & 1 & -1 & 0 & 0 & 0 & 0 & 0 & 0 \\
0 & 0 & 0 & 1 & -1 & 0 & 0 & 0 & 0
\end{array}\right)
$$

de modo que, $A \theta-\mathbf{b}=0$, de onde obtemos: 


$$
\begin{gathered}
A \hat{\theta}=\left(\begin{array}{ll}
-1.3379 & 0.071690
\end{array}\right)^{\prime} \mathrm{e} \\
{\left[A \hat{I}_{F}^{-1}(\hat{\theta}) A^{\prime}\right]^{-1}=\left(\begin{array}{ll}
26.668 & 516.30 \\
516.30 & 10210 .
\end{array}\right) .}
\end{gathered}
$$

Neste caso o valor da estatística $\mathrm{W}=1.1710$, que indica a não rejeição de $\mathrm{H}_{0}$, isto é, não existe diferencas entre os vícios.

\section{Instrumentos 3 e 5:}

No caso de testar a hipótese:

$$
H_{01}: \alpha_{2}=\alpha_{3}=0, \beta_{2}=\beta_{3}=1 ;
$$

que pode ser escrita como $H_{01}: A \theta=\mathbf{b}$, com a matriz $\mathrm{A}$ e vetor $\mathbf{b}$ dados por:

$$
A=\left(\begin{array}{ccccccccc}
0 & 1 & 0 & 0 & 0 & 0 & 0 & 0 & 0 \\
0 & 0 & 1 & 0 & 0 & 0 & 0 & 0 & 0 \\
0 & 0 & 0 & 1 & 0 & 0 & 0 & 0 & 0 \\
0 & 0 & 0 & 0 & 1 & 0 & 0 & 0 & 0
\end{array}\right) \text { e } \mathbf{b}=\left(\begin{array}{c}
0 \\
0 \\
1 \\
1
\end{array}\right)
$$

de modo que, temos para cada caso:

$$
A \hat{\theta}-\mathbf{b}=\left(\begin{array}{llll}
0.084098 & 1.9255 & -0.0048604 & -0.10772
\end{array}\right)^{\prime},
$$

com:

$$
\left[A \hat{I}_{F}^{-1}(\hat{\theta}) A^{\prime}\right]^{-1}=\left(\begin{array}{cccc}
19.232 & 0.80599 & 372.33 & 15.604 \\
0.80599 & 17.974 & 15.604 & 347.97 \\
372.33 & 15.604 & 7368.1 & 297.58 \\
15.604 & 347.97 & 297.58 & 6888.8
\end{array}\right)
$$

que leva ao valor da estatística $\mathrm{W}=2.2277$.

Comparando $\mathrm{W}$ com o valor crítico de qui-quadrado a um nível de significância de $5 \%$ com 4 graus de liberdade, $\chi_{s=4}^{2}(0,05)=9.488$, temos que $2.2277<9.4882$, então não rejeitamos a hipótese nula o que significa que os instrumentos não têm vícios aditivos e multiplicativos, ou seja, que nenhum instrumento tem viés aditivo não nulo ou vício multiplicativo diferente de um. 
Agora, passemos a verificar se esses vícios aditivos e multiplicativos são todos iguais entre eles. Para este caso a hipótese de interesse é dada por:

$$
H_{02}: \alpha_{2}=\alpha_{3}=\alpha, \beta_{2}=\beta_{3}=\beta ;
$$

onde a matriz A é dada por:

$$
A=\left(\begin{array}{ccccccccc}
0 & 1 & -1 & 0 & 0 & 0 & 0 & 0 & 0 \\
0 & 0 & 0 & 1 & -1 & 0 & 0 & 0 & 0
\end{array}\right),
$$

de modo que, $A \theta-\mathbf{b}=0$, de onde obtemos:

$$
\begin{gathered}
A \hat{\theta}=\left(\begin{array}{ll}
-1.8414 & 0.10286
\end{array}\right)^{\prime} \mathrm{e} \\
{\left[\begin{array}{l}
\left.A \hat{I}_{F}^{-1}(\hat{\theta}) A^{\prime}\right]^{-1} \\
\end{array}=\left(\begin{array}{ll}
8.8883 & 172.08 \\
172.08 & 3411.6
\end{array}\right) .\right.}
\end{gathered}
$$

Neste caso o valor da estatística $\mathrm{W}=1.0479$, que indica a não rejeição de $\mathrm{H}_{0}$, isto é, não existe diferencas entre os vícios.

\section{Caso 4 e 5:}

No caso de testar a hipótese:

$$
H_{01}: \alpha_{2}=\alpha_{3}=0, \beta_{2}=\beta_{3}=1 ;
$$

que pode ser escrita como $H_{01}: A \theta=\mathbf{b}$, com a matriz $\mathrm{A}$ e vetor $\mathbf{b}$ dados por:

$$
A=\left(\begin{array}{ccccccccc}
0 & 1 & 0 & 0 & 0 & 0 & 0 & 0 & 0 \\
0 & 0 & 1 & 0 & 0 & 0 & 0 & 0 & 0 \\
0 & 0 & 0 & 1 & 0 & 0 & 0 & 0 & 0 \\
0 & 0 & 0 & 0 & 1 & 0 & 0 & 0 & 0
\end{array}\right) \text { e } \mathbf{b}=\left(\begin{array}{c}
0 \\
0 \\
1 \\
1
\end{array}\right)
$$

de modo que, temos para cada caso:

$$
A \hat{\theta}-\mathbf{b}=\left(\begin{array}{llll}
0.74104 & 1.3399 & -0.41376 & -0.077477
\end{array}\right)^{\prime}
$$

com:

$$
\left[A \hat{I}_{F}^{-1}(\hat{\theta}) A^{\prime}\right]^{-1}=\left(\begin{array}{cccc}
15.929 & -6.7042 & 308.38 & -129.79 \\
-6.7042 & 20.546 & -129.79 & 397.77 \\
308.38 & -129.79 & 6104.2 & -2570.7 \\
-129.79 & 397.77 & -2570.7 & 7871.3
\end{array}\right)
$$


que leva ao valor da estatística $\mathrm{W}=1.3361$.

Comparando $\mathrm{W}$ com o valor crítico de qui-quadrado a um nível de significância de $5 \%$ com 4 graus de liberdade, $\chi_{s=4}^{2}(0,05)=9.488$, temos que $1.3361<9.4882$, então não rejeitamos a hipótese nula o que significa que os instrumentos não têm vícios aditivos e multiplicativos, ou seja, que nenhum instrumento tem viés aditivo não nulo vício multiplicativo diferente de um.

Agora, passemos a verificar se esses vícios aditivos e multiplicativos são todos iguais entre eles. Para este caso a hipótese de interesse é dada por:

$$
H_{02}: \alpha_{2}=\alpha_{3}=\alpha, \beta_{2}=\beta_{3}=\beta ;
$$

onde a matriz A é dada por:

$$
A=\left(\begin{array}{ccccccccc}
0 & 1 & -1 & 0 & 0 & 0 & 0 & 0 & 0 \\
0 & 0 & 0 & 1 & -1 & 0 & 0 & 0 & 0
\end{array}\right),
$$

de modo que, $A \theta-b=0$, de onde obtemos:

$$
\begin{aligned}
& A \hat{\theta}=\left(\begin{array}{ll}
-0.59891 & 0.36101
\end{array}\right)^{\prime} \mathrm{e} \\
& {\left[A \hat{I}_{F}^{-1}(\hat{\theta}) A^{\prime}\right]^{-1}=\left(\begin{array}{ll}
12.240 & 236.96 \\
236.96 & 4690.9
\end{array}\right) .}
\end{aligned}
$$

Neste caso o valor da estatística $\mathrm{W}=0.25703$, que indica a não rejeição de $\mathrm{H}_{0}$, isto é, não existe diferencas entre os vícios.

Passemos agora a mostrar os resultados para o teste de Wald em que foram utilizandos quatro instrumentos de cada vez.

Obtemos o seguinte resultado para a matriz de informação de Fisher estimada $\hat{I}$ e para sua inversa $\hat{I}^{-1}$ :

\section{Instrumentos 2, 3 e 4:}

$$
\begin{aligned}
& \hat{I}= \\
& \begin{array}{llllll}
1.1345 & 0.44418 & 0.17058 & 0.19776 & 8.5994 & 3.3024
\end{array} \\
& \begin{array}{llllll}
0.44418 & 39.657 & -10.322 & -11.967 & 767.77 & -199.84
\end{array} \\
& \begin{array}{llllll}
0.17058 & -10.322 & 22.112 & -4.5957 & -199.84 & 428.08
\end{array}
\end{aligned}
$$




$\begin{array}{rrrrrr}0.19776 & -11.967 & -4.5957 & 26.541 & -231.69 & -88.973 \\ 8.5994 & 767.77 & -199.84 & -231.69 & 15211 . & -3958.2 \\ 3.3024 & -199.84 & 428.08 & -88.973 & -3958.2 & 8480.3 \\ 3.8286 & -231.69 & -88.973 & 513.84 & -4588.9 & -1762.3 \\ 0.00000 & 0.00000 & 0.00000 & 0.00000 & 0.44133 & 0.16948 \\ 0.00000 & 0.00000 & 0.00000 & 0.00000 & -5.0515 & -1.9399 \\ 0.00000 & 0.00000 & 0.00000 & 0.00000 & 15.428 & -4.0157 \\ 0.00000 & 0.00000 & 0.00000 & 0.00000 & -1.5421 & 3.3034 \\ 0.00000 & 0.00000 & 0.00000 & 0.00000 & -2.0727 & -0.79598 \\ & & & & & \\ 3.8286 & 0.00000 & 0.00000 & 0.00000 & 0.00000 & 0.00000 \\ -231.69 & 0.00000 & 0.00000 & 0.00000 & 0.00000 & 0.00000 \\ -88.973 & 0.00000 & 0.00000 & 0.00000 & 0.00000 & 0.00000 \\ 513.84 & 0.00000 & 0.00000 & 0.00000 & 0.00000 & 0.00000 \\ -4588.9 & 0.44133 & -5.0515 & 15.428 & -1.5421 & -2.0727 \\ -1762.3 & 0.16948 & -1.9399 & -4.0157 & 3.3034 & -0.79598 \\ 10179 . & 0.19649 & -2.2490 & -4.6555 & -0.68658 & 4.5970 \\ 0.19649 & 0.064349 & 0.0047655 & 0.0051391 & 0.0049347 & 0.0044402 \\ -2.2490 & 0.0047655 & 61.386 & 17.452 & 2.5737 & 3.4592 \\ -4.6555 & 0.0051391 & 17.452 & 78.635 & 5.3277 & 7.1607 \\ -0.68658 & 0.0049347 & 2.5737 & 5.3277 & 24.446 & 1.0560 \\ 4.5970 & 0.0044402 & 3.4592 & 7.1607 & 1.0560 & 35.222 \\ \hat{I}^{-1}= & & & & & \end{array}$

$\begin{array}{rrrrrr}0.89664 & -0.021624 & -0.021189 & -0.020100 & -9.2351 e-016 & -4.2887 e-016 \\ -0.021624 & 1.6737 & 0.97800 & 0.92764 & -0.084516 & -0.049380 \\ -0.021189 & 0.97800 & 2.6520 & 0.90794 & -0.049380 & -0.13389 \\ -0.020100 & 0.92764 & 0.90794 & 2.2486 & -0.046837 & -0.045841 \\ -6.2058 \mathrm{e}-018 & -0.084516 & -0.049380 & -0.046837 & 0.0043655 & 0.0025506 \\ -2.5816 \mathrm{e}-018 & -0.049380 & -0.13389 & -0.045841 & 0.0025506 & 0.0069157 \\ -2.7154 \mathrm{e}-017 & -0.046837 & -0.045841 & -0.11352 & 0.0024193 & 0.0023678 \\ 1.3225 \mathrm{e}-016 & 0.85285 & 0.83085 & 0.78845 & -0.044052 & -0.042916 \\ -1.6363 \mathrm{e}-018 & -0.014304 & -0.011096 & -0.010572 & 0.00073883 & 0.00057313 \\ -4.2624 \mathrm{e}-019 & 0.014672 & 0.0015468 & 0.0013439 & -0.00075784 & -7.9895 e-005 \\ -7.1247 \mathrm{e}-019 & -0.0017686 & 0.014340 & 0.00020779 & 9.1352 \mathrm{e}-005 & -0.00074072 \\ 3.3724 \mathrm{e}-018 & -0.0016091 & 0.00029192 & 0.011683 & 8.3112 \mathrm{e}-005 & -1.5078 \mathrm{e}-005 \\ & & & & & \\ 1.6351 \mathrm{e}-016 & 3.2064 \mathrm{e}-015 & 1.5540 \mathrm{e}-017 & 8.7491 \mathrm{e}-017 & 1.6016 \mathrm{e}-017 & 6.0230 \mathrm{e}-018 \\ -0.046837 & 0.85285 & -0.014304 & 0.014672 & -0.0017686 & -0.0016091 \\ -0.045841 & 0.83085 & -0.011096 & 0.0015468 & 0.014340 & 0.00029192 \\ -0.11352 & 0.78845 & -0.010572 & 0.0013439 & 0.00020779 & 0.011683 \\ 0.0024193 & -0.044052 & 0.00073883 & -0.00075784 & 9.1352 \mathrm{e}-005 & 8.3112 \mathrm{e}-005\end{array}$




$\begin{array}{rrrrrr}0.0023678 & -0.042916 & 0.00057313 & -7.9895 e-005 & -0.00074072 & -1.5078 e-005 \\ 0.0058638 & -0.040726 & 0.00054609 & -6.9416 e-005 & -1.0733 e-005 & -0.00060348 \\ -0.040726 & 16.080 & -0.0090995 & 0.0051460 & -0.0015166 & -0.00038105 \\ 0.00054609 & -0.0090995 & 0.017573 & -0.0038310 & -0.00098862 & -0.00093106 \\ -6.9416 e-005 & 0.0051460 & -0.0038310 & 0.014107 & -0.0026052 & -0.0024516 \\ -1.0733 e-005 & -0.0015166 & -0.00098862 & -0.0026052 & 0.041711 & -0.00063363 \\ -0.00060348 & -0.00038105 & -0.00093106 & -0.0024516 & -0.00063363 & 0.029083\end{array}$

Instrumentos 2, 3 e 5:

$\hat{I}=$

\begin{tabular}{rrrrrr}
1.1169 & 0.16351 & 0.079283 & 0.066110 & 3.1655 & 1.5349 \\
0.16351 & 35.546 & -3.0264 & -2.5235 & 688.17 & -58.591 \\
0.079283 & -3.0264 & 19.227 & -1.2236 & -58.591 & 372.23 \\
0.066110 & -2.5235 & -1.2236 & 18.215 & -48.855 & -23.690 \\
3.1655 & 688.17 & -58.591 & -48.855 & 13639 & -1161.1 \\
1.5349 & -58.591 & 372.23 & -23.690 & -1161.1 & 7377.2 \\
1.2799 & -48.855 & -23.690 & 352.64 & -968.17 & -469.46 \\
0.00000 & 0.00000 & 0.00000 & 0.00000 & 0.16288 & 0.078980 \\
0.00000 & 0.00000 & 0.00000 & 0.00000 & -22.363 & -10.844 \\
0.00000 & 0.00000 & 0.00000 & 0.00000 & 5.1838 & -0.44135 \\
0.00000 & 0.00000 & 0.00000 & 0.00000 & -0.21401 & 1.3596 \\
0.00000 & 0.00000 & 0.00000 & 0.00000 & -0.14880 & -0.072152 \\
1.2799 & 0.00000 & 0.00000 & 0.00000 & 0.00000 & 0.00000 \\
-48.855 & 0.00000 & 0.00000 & 0.00000 & 0.00000 & 0.00000 \\
-23.690 & 0.00000 & 0.00000 & 0.00000 & 0.00000 & 0.00000 \\
352.64 & 0.00000 & 0.00000 & 0.00000 & 0.00000 & 0.00000 \\
-968.17 & 0.16288 & -22.363 & 5.1838 & -0.21401 & -0.14880 \\
-469.46 & 0.078980 & -10.844 & -0.44135 & 1.3596 & -0.072152 \\
6989.0 & 0.065857 & -9.0418 & -0.36802 & -0.086530 & 1.0740 \\
0.065857 & 0.062372 & 0.032842 & 0.034448 & 0.033026 & 0.026578 \\
-9.0418 & 0.032842 & 172.81 & 47.853 & 11.251 & 7.8230 \\
-0.36802 & 0.034448 & 47.853 & 63.175 & 0.45795 & 0.31841 \\
-0.086530 & 0.033026 & 11.251 & 0.45795 & 18.483 & 0.074865 \\
1.0740 & 0.026578 & 7.8230 & 0.31841 & 0.074865 & 16.589 \\
\hline$I^{-1}=$ & & & & & \\
-0.0048280 & 1.2762 & 0.23395 & 0.20954 & -0.064429 & -0.011834
\end{tabular}




$\begin{array}{rrrrrr}-0.0047272 & 0.23395 & 2.3152 & 0.20343 & -0.011834 & -0.11684 \\ -0.0042407 & 0.20954 & 0.20343 & 2.4283 & -0.010599 & -0.010288 \\ 2.4953 e-018 & -0.064429 & -0.011834 & -0.010599 & 0.0033279 & 0.00061127 \\ -3.5742 \mathrm{e}-019 & -0.011834 & -0.11684 & -0.010288 & 0.00061127 & 0.0060353 \\ -3.2084 \mathrm{e}-019 & -0.010599 & -0.010288 & -0.12254 & 0.00054746 & 0.00053140 \\ -5.4903 \mathrm{e}-018 & 0.18607 & 0.18026 & 0.16234 & -0.0096112 & -0.0093110 \\ 4.5708 \mathrm{e}-019 & -0.015040 & -0.013548 & -0.011965 & 0.00077684 & 0.00069979 \\ -5.5012 \mathrm{e}-019 & 0.016339 & 0.010112 & 0.0089440 & -0.00084394 & -0.00052234 \\ -2.0050 \mathrm{e}-019 & 0.0084664 & 0.016060 & 0.0067797 & -0.00043731 & -0.00082956 \\ -1.5369 \mathrm{e}-019 & 0.0064992 & 0.0058852 & 0.012974 & -0.00033570 & -0.00030399 \\ & & & & & \\ 3.7378 \mathrm{e}-016 & 4.1211 \mathrm{e}-015 & -6.5449 \mathrm{e}-017 & 1.6209 \mathrm{e}-016 & 2.9900 \mathrm{e}-016 & -1.8162 \mathrm{e}-016 \\ -0.010599 & 0.18607 & -0.015040 & 0.016339 & 0.0084664 & 0.0064992 \\ -0.010288 & 0.18026 & -0.013548 & 0.010112 & 0.016060 & 0.0058852 \\ -0.12254 & 0.16234 & -0.011965 & 0.0089440 & 0.0067797 & 0.012974 \\ 0.00054746 & -0.0096112 & 0.00077684 & -0.00084394 & -0.00043731 & -0.00033570 \\ 0.00053140 & -0.0093110 & 0.00069979 & -0.00052234 & -0.00082956 & -0.00030399 \\ 0.0063297 & -0.0083853 & 0.00061803 & -0.00046198 & -0.00035019 & -0.00067015 \\ -0.0083853 & 16.108 & -0.00029571 & -0.0075576 & -0.027779 & -0.024982 \\ 0.00061803 & -0.00029571 & 0.0081593 & -0.0061816 & -0.0048376 & -0.0037367 \\ -0.00046198 & -0.0075576 & -0.0061816 & 0.020542 & 0.0032837 & 0.0025381 \\ -0.00035019 & -0.027779 & -0.0048376 & 0.0032837 & 0.057062 & 0.0020204 \\ -0.00067015 & -0.024982 & -0.0037367 & 0.0025381 & 0.0020204 & 0.062064\end{array}$

Instrumentos 2, 4 e 5:

$\hat{I}=$

$\begin{array}{rrrrrr}2.7343 & 1.6982 & -0.0065713 & -0.0037971 & 32.878 & -0.12722 \\ 1.6982 & 36.252 & -22.889 & -13.226 & 701.83 & -443.14 \\ -0.0065713 & -22.889 & 24.895 & 0.051178 & -443.14 & 481.97 \\ -0.0037971 & -13.226 & 0.051178 & 15.329 & -256.06 & 0.99081 \\ 32.878 & 701.83 & -443.14 & -256.06 & 13724 . & -8662.9 \\ -0.12722 & -443.14 & 481.97 & 0.99081 & -8662.9 & 9421.9 \\ -0.073511 & -256.06 & 0.99081 & 296.77 & -5005.7 & 19.369 \\ 0.00000 & 0.00000 & 0.00000 & 0.00000 & 1.6985 & -0.0065724 \\ 0.00000 & 0.00000 & 0.00000 & 0.00000 & 0.0022352 & -8.6490 \mathrm{e}-006 \\ 0.00000 & 0.00000 & 0.00000 & 0.00000 & 22.519 & -14.219 \\ 0.00000 & 0.00000 & 0.00000 & 0.00000 & 0.055019 & -0.059840 \\ 0.00000 & 0.00000 & 0.00000 & 0.00000 & 0.018370 & -7.1082 \mathrm{e}-005 \\ -0.073511 & 0.00000 & 0.00000 & 0.00000 & 0.00000 & 0.00000 \\ -256.06 & 0.00000 & 0.00000 & 0.00000 & 0.00000 & 0.00000 \\ 0.99081 & 0.00000 & 0.00000 & 0.00000 & 0.00000 & 0.00000\end{array}$




\begin{tabular}{|c|c|c|c|c|c|}
\hline 296.77 & 0.00000 & 0.00000 & 0.00000 & 0.00000 & 0.00000 \\
\hline-5005.7 & 1.6985 & 0.0022352 & 22.519 & 0.055019 & 0.018370 \\
\hline 19.369 & -0.0065724 & $-8.6490 e-006$ & -14.219 & -0.059840 & $-7.1082 e-005$ \\
\hline 5801.5 & -0.0037977 & $-4.9976 e-006$ & -8.2159 & -0.00012302 & -0.021290 \\
\hline-0.0037977 & 0.37383 & $8.7714 \mathrm{e}-008$ & $2.3012 e-007$ & $1.9354 \mathrm{e}-007$ & $1.6988 \mathrm{e}-007$ \\
\hline$-4.9976 e-006$ & $8.7714 \mathrm{e}-008$ & 2.7607 & 1.0642 & $1.5935 e-005$ & $5.3204 e-006$ \\
\hline-8.2159 & $2.3012 e-007$ & 1.0642 & 65.710 & 26.196 & 8.7465 \\
\hline-0.00012302 & $1.9354 \mathrm{e}-007$ & $1.5935 \mathrm{e}-005$ & 26.196 & 30.988 & 0.00013096 \\
\hline-0.021290 & $1.6988 \mathrm{e}-007$ & $5.3204 e-006$ & 8.7465 & 0.00013096 & 11.749 \\
\hline \multicolumn{6}{|l|}{$\hat{I}^{-1}=$} \\
\hline 0.50043 & -0.21809 & -0.20000 & -0.18738 & $1.5090 e-013$ & $1.3661 e-013$ \\
\hline-0.21809 & 37.146 & 33.668 & 31.542 & -1.9004 & -1.7223 \\
\hline-0.20000 & 33.668 & 34.942 & 28.830 & -1.7223 & -1.7874 \\
\hline-0.18738 & 31.542 & 28.830 & 33.772 & -1.6136 & -1.4748 \\
\hline $1.5024 \mathrm{e}-013$ & -1.9004 & -1.7223 & -1.6136 & 0.098164 & 0.088961 \\
\hline $1.3596 e-013$ & -1.7223 & -1.7874 & -1.4748 & 0.088961 & 0.092326 \\
\hline $1.2767 e-013$ & -1.6136 & -1.4748 & -1.7276 & 0.083346 & 0.076176 \\
\hline$-6.7895 e-013$ & 8.5883 & 7.7791 & 7.2880 & -0.44361 & -0.40181 \\
\hline $4.0975 e-015$ & -0.051576 & -0.011891 & -0.011086 & 0.0026641 & 0.00061419 \\
\hline$-1.0943 e-014$ & 0.13776 & 0.034440 & 0.032126 & -0.0071157 & -0.0017789 \\
\hline $9.2471 \mathrm{e}-015$ & -0.11642 & -0.029514 & -0.027148 & 0.0060132 & 0.0015245 \\
\hline $8.1438 \mathrm{e}-015$ & -0.10252 & -0.025630 & -0.024533 & 0.0052955 & 0.0013239 \\
\hline $1.2851 \mathrm{e}-013$ & $-6.7730 e-013$ & $4.1844 \mathrm{e}-015$ & $-1.1123 e-014$ & $9.4507 e-015$ & $8.3732 e-015$ \\
\hline-1.6136 & 8.5883 & -0.051576 & 0.13776 & -0.11642 & -0.10252 \\
\hline-1.4748 & 7.7791 & -0.011891 & 0.034440 & -0.029514 & -0.025630 \\
\hline-1.7276 & 7.2880 & -0.011086 & 0.032126 & -0.027148 & -0.024533 \\
\hline 0.083346 & -0.44361 & 0.0026641 & -0.0071157 & 0.0060132 & 0.0052955 \\
\hline 0.076176 & -0.40181 & 0.00061419 & -0.0017789 & 0.0015245 & 0.0013239 \\
\hline 0.089234 & -0.37645 & 0.00057263 & -0.0016594 & 0.0014023 & 0.0012672 \\
\hline-0.37645 & 4.6798 & -0.012088 & 0.032283 & -0.027281 & -0.024025 \\
\hline 0.00057263 & -0.012088 & 0.36677 & -0.011788 & 0.0099612 & 0.0087722 \\
\hline-0.0016594 & 0.032283 & -0.011788 & 0.030593 & -0.025853 & -0.022767 \\
\hline 0.0014023 & -0.027281 & 0.0099612 & -0.025853 & 0.054118 & 0.019239 \\
\hline 0.0012672 & -0.024025 & 0.0087722 & -0.022767 & 0.019239 & 0.10206 \\
\hline
\end{tabular}

Instrumentos 3, 4 e 5:

$\hat{I}=$ 


\begin{tabular}{|c|c|c|c|c|c|}
\hline 1.1471 & 0.42460 & 0.32686 & 0.13229 & 8.2203 & 6.3280 \\
\hline 0.42460 & 29.139 & -13.713 & -5.5503 & 564.12 & -265.49 \\
\hline 0.32686 & -13.713 & 27.780 & -4.2727 & -265.49 & 537.82 \\
\hline 0.13229 & -5.5503 & -4.2727 & 14.916 & -107.45 & -82.719 \\
\hline 8.2203 & 564.12 & -265.49 & -107.45 & 11172 . & -5256.3 \\
\hline 6.3280 & -265.49 & 537.82 & -82.719 & -5256.3 & 10651 \\
\hline 2.5612 & -107.45 & -82.719 & 288.76 & -2127.4 & -1637.7 \\
\hline 0.00000 & 0.00000 & 0.00000 & 0.00000 & 0.42089 & 0.32400 \\
\hline 0.00000 & 0.00000 & 0.00000 & 0.00000 & -2.6265 & -2.0219 \\
\hline 0.00000 & 0.00000 & 0.00000 & 0.00000 & 10.691 & -5.0316 \\
\hline 0.00000 & 0.00000 & 0.00000 & 0.00000 & -3.8734 & 7.8465 \\
\hline 0.00000 & 0.00000 & 0.00000 & 0.00000 & -0.63452 & -0.48846 \\
\hline 2.5612 & 0.00000 & 0.00000 & 0.00000 & 0.00000 & 0.00000 \\
\hline-107.45 & 0.00000 & 0.00000 & 0.00000 & 0.00000 & 0.00000 \\
\hline-82.719 & 0.00000 & 0.00000 & 0.00000 & 0.00000 & 0.00000 \\
\hline 288.76 & 0.00000 & 0.00000 & 0.00000 & 0.00000 & 0.00000 \\
\hline-2127.4 & 0.42089 & -2.6265 & 10.691 & -3.8734 & -0.63452 \\
\hline-1637.7 & 0.32400 & -2.0219 & -5.0316 & 7.8465 & -0.48846 \\
\hline 5718.4 & 0.13114 & -0.81834 & -2.0365 & -1.2068 & 1.7052 \\
\hline 0.13114 & 0.065791 & 0.0036223 & 0.0038729 & 0.0034427 & 0.0029917 \\
\hline-0.81834 & 0.0036223 & 27.890 & 6.3758 & 3.7783 & 0.61895 \\
\hline-2.0365 & 0.0038729 & 6.3758 & 42.453 & 9.4026 & 1.5403 \\
\hline-1.2068 & 0.0034427 & 3.7783 & 9.4026 & 38.586 & 0.91279 \\
\hline 1.7052 & 0.0029917 & 0.61895 & 1.5403 & 0.91279 & 11.124 \\
\hline \multicolumn{6}{|l|}{$\hat{I}^{-1}=$} \\
\hline 0.89664 & -0.033598 & -0.031677 & -0.029530 & $5.6062 \mathrm{e}-016$ & $1.5941 \mathrm{e}-016$ \\
\hline-0.033598 & 2.5422 & 1.4766 & 1.3781 & -0.12842 & -0.074578 \\
\hline-0.031677 & 1.4766 & 2.5725 & 1.2983 & -0.074578 & -0.12994 \\
\hline-0.029530 & 1.3781 & 1.2983 & 3.9048 & -0.069604 & -0.065576 \\
\hline $2.2269 \mathrm{e}-017$ & -0.12842 & -0.074578 & -0.069604 & 0.0066331 & 0.0038522 \\
\hline $4.5800 e-017$ & -0.074578 & -0.12994 & -0.065576 & 0.0038522 & 0.0067116 \\
\hline $1.8963 e-017$ & -0.069604 & -0.065576 & -0.19721 & 0.0035952 & 0.0033872 \\
\hline$-4.0590 e-016$ & 1.3277 & 1.2479 & 1.1611 & -0.068581 & -0.064460 \\
\hline $6.7068 e-018$ & -0.024819 & -0.020884 & -0.018320 & 0.0012820 & 0.0010787 \\
\hline $1.3921 \mathrm{e}-018$ & 0.024627 & -0.00094447 & 0.0018929 & -0.0012721 & $4.8785 e-005$ \\
\hline$-7.4574 e-018$ & -0.0035435 & 0.019061 & 0.00084709 & 0.00018303 & -0.00098458 \\
\hline $5.2968 e-019$ & -0.0020258 & -0.00051461 & 0.023756 & 0.00010464 & $2.6581 e-005$ \\
\hline $1.1663 e-016$ & $-1.7072 e-01$ & $46.0076 e-017$ & $-4.0912 e-017$ & $7-2.1621 e-017$ & $-4.0542 e-017$ \\
\hline-0.069604 & $\quad 1.327$ & $7 \quad-0.024819$ & 0.024627 & $7 \quad-0.0035435$ & -0.0020258 \\
\hline
\end{tabular}




$\begin{array}{rrrrrr}-0.065576 & 1.2479 & -0.020884 & -0.00094447 & 0.019061 & -0.00051461 \\ -0.19721 & 1.1611 & -0.018320 & 0.0018929 & 0.00084709 & 0.023756 \\ 0.0035952 & -0.068581 & 0.0012820 & -0.0012721 & 0.00018303 & 0.00010464 \\ 0.0033872 & -0.064460 & 0.0010787 & 4.8785 \mathrm{e}-005 & -0.00098458 & 2.6581 \mathrm{e}-005 \\ 0.010186 & -0.059973 & 0.00094630 & -9.7776 \mathrm{e}-005 & -4.3755 \mathrm{e}-005 & -0.0012271 \\ -0.059973 & 16.076 & -0.016983 & 0.0072858 & 0.0028525 & -0.0021709 \\ 0.00094630 & -0.016983 & 0.037634 & -0.0052152 & -0.0024456 & -0.0011912 \\ -9.7776 \mathrm{e}-005 & 0.0072858 & -0.0052152 & 0.026075 & -0.0059161 & -0.0028923 \\ -4.3755 \mathrm{e}-005 & 0.0028525 & -0.0024456 & -0.0059161 & 0.027846 & -0.0013566 \\ -0.0012271 & -0.0021709 & -0.0011912 & -0.0028923 & -0.0013566 & 0.090673\end{array}$

\section{Instrumentos 2, 3 e 4:}

No caso de testar a hipótese:

$$
H_{01}: \alpha_{2}=\alpha_{3}=\alpha_{4}=0, \beta_{2}=\beta_{3}=\beta_{4}=1 ;
$$

que pode ser escrita como $H_{01}: A \theta=b$, com a matriz $\mathrm{A}$ e vetor $\mathbf{b}$ dados por:

$$
A=\left(\begin{array}{cccccccccccc}
0 & 1 & 0 & 0 & 0 & 0 & 0 & 0 & 0 & 0 & 0 & 0 \\
0 & 0 & 1 & 0 & 0 & 0 & 0 & 0 & 0 & 0 & 0 & 0 \\
0 & 0 & 0 & 1 & 0 & 0 & 0 & 0 & 0 & 0 & 0 & 0 \\
0 & 0 & 0 & 0 & 1 & 0 & 0 & 0 & 0 & 0 & 0 & 0 \\
0 & 0 & 0 & 0 & 0 & 1 & 0 & 0 & 0 & 0 & 0 & 0 \\
0 & 0 & 0 & 0 & 0 & 0 & 1 & 0 & 0 & 0 & 0 & 0
\end{array}\right) \text { e } \mathbf{b}=\left(\begin{array}{c}
0 \\
0 \\
0 \\
1 \\
1 \\
1
\end{array}\right)
$$

de modo que, temos para cada caso:

$$
A \hat{\theta}-\mathbf{b}=\left(\begin{array}{llllll}
0.20562 & -0.35055 & 0.61257 & 0.038449 & 0.017590 & 0.034740
\end{array}\right)^{\prime},
$$

com:

$$
\left[A \hat{I}_{F}^{-1}(\hat{\theta}) A^{\prime}\right]^{-1}=\left(\begin{array}{cccccc}
39.483 & -10.389 & -12.045 & 764.40 & -201.14 & -233.18 \\
-10.389 & 22.086 & -4.6255 & -201.14 & 427.58 & -89.549 \\
-12.045 & -4.6255 & 26.507 & -233.18 & -89.549 & 513.17 \\
764.40 & -201.14 & -233.18 & 15138 . & -3983.5 & -4618.1 \\
-201.14 & 427.58 & -89.549 & -3983.5 & 8469.4 & -1774.1 \\
-233.18 & -89.549 & 513.17 & -4618.1 & -1774.1 & 10165 .
\end{array}\right)
$$

que leva ao valor da estatística $\mathrm{W}=38.339$.

Comparando $\mathrm{W}$ com o valor crítico de qui-quadrado a um nível de significância de $5 \%$ com 6 graus de liberdade, $\chi_{s=4}^{2}(0,05)=12.59$, temos que $38.339>12.59$, então rejeitamos a hipótese nula o que significa que os instrumentos têm vícios aditivos e multiplicativos, ou seja, que pelo menos um instrumento tem viés aditivo não nulo ou 
pelo menos um deles tem vício multiplicativo diferente de um.

Agora, passemos a verificar se esses vícios aditivos e multiplicativos são todos iguais entre eles. Para este caso a hipótese de interesse é dada por:

$$
H_{02}: \alpha_{2}=\alpha_{3}=\alpha_{4}=\alpha, \beta_{2}=\beta_{3}=\beta_{4}=\beta ;
$$

onde a matriz A é dada por:

$$
A=\left(\begin{array}{cccccccccccc}
0 & 1 & -1 & 0 & 0 & 0 & 0 & 0 & 0 & 0 & 0 & 0 \\
0 & 0 & 1 & -1 & 0 & 0 & 0 & 0 & 0 & 0 & 0 & 0 \\
0 & 0 & 0 & 0 & 1 & -1 & 0 & 0 & 0 & 0 & 0 & 0 \\
0 & 0 & 0 & 0 & 0 & 1 & -1 & 0 & 0 & 0 & 0 & 0
\end{array}\right)
$$

de modo que, $A \theta-\mathbf{b}=0$, de onde obtemos:

$$
\begin{aligned}
& A \hat{\theta}=\left(\begin{array}{llll}
0.55617 & -0.96312 & 0.020859 & 0.052331
\end{array}\right)^{\prime} \mathrm{e} \\
& {\left[A \hat{I}_{F}^{-1}(\hat{\theta}) A^{\prime}\right]^{-1}=\left(\begin{array}{cccc}
30.923 & 16.984 & 598.67 & 328.80 \\
16.984 & 23.657 & 328.80 & 458.01 \\
598.67 & 328.80 & 11857 . & 6511.8 \\
328.80 & 458.01 & 6511.8 & 9071.6
\end{array}\right)}
\end{aligned}
$$

Neste caso o valor da estatística $\mathrm{W}=31.184$, que indica rejeição de $\mathrm{H}_{0}$, isto é, existe diferencas entre os vícios.

\section{Instrumentos 2, 3 e 5:}

No caso de testar a hipótese:

$$
H_{01}: \alpha_{2}=\alpha_{3}=\alpha_{4}=0, \beta_{2}=\beta_{3}=\beta_{4}=1 ;
$$

que pode ser escrita como $H_{01}: A \theta=\mathbf{b}$, com a matriz $\mathrm{A}$ e vetor $\mathbf{b}$ dados por:

$$
A=\left(\begin{array}{cccccccccccc}
0 & 1 & 0 & 0 & 0 & 0 & 0 & 0 & 0 & 0 & 0 & 0 \\
0 & 0 & 1 & 0 & 0 & 0 & 0 & 0 & 0 & 0 & 0 & 0 \\
0 & 0 & 0 & 1 & 0 & 0 & 0 & 0 & 0 & 0 & 0 & 0 \\
0 & 0 & 0 & 0 & 1 & 0 & 0 & 0 & 0 & 0 & 0 & 0 \\
0 & 0 & 0 & 0 & 0 & 1 & 0 & 0 & 0 & 0 & 0 & 0 \\
0 & 0 & 0 & 0 & 0 & 0 & 1 & 0 & 0 & 0 & 0 & 0
\end{array}\right) \text { e } \mathbf{b}=\left(\begin{array}{c}
0 \\
0 \\
0 \\
1 \\
1 \\
1
\end{array}\right)
$$

de modo que, temos para cada caso: 


$$
A \hat{\theta}-\mathbf{b}=\left(\begin{array}{llllll}
0.48211 & -0.064202 & 1.7838 & 0.024168 & 0.0027997 & -0.10040
\end{array}\right)^{\prime},
$$

com:

$$
\begin{aligned}
& {\left[A \hat{I}_{F}^{-1}(\hat{\theta}) A^{\prime}\right]^{-1}=} \\
& \left(\begin{array}{ccccccc}
35.522 & -3.0380 & -2.5332 & 687.70 & -58.815 & -49.043 \\
-3.0380 & 19.221 & -1.2283 & -58.815 & 372.12 & -23.781 \\
-2.5332 & -1.2283 & 18.211 & -49.043 & -23.781 & 352.56 \\
687.70 & -58.815 & -49.043 & 13624 . & -1167.9 & -973.81 \\
-58.815 & 372.12 & -23.781 & -1167.9 & 7373.9 & -472.11 \\
-49.043 & -23.781 & 352.56 & -973.81 & -472.11 & 6986.7
\end{array}\right),
\end{aligned}
$$

que leva ao valor da estatística $\mathrm{W}=35.277$.

Comparando $\mathrm{W}$ com o valor crítico de qui-quadrado a um nível de significância de $5 \%$ com 6 graus de liberdade, $\chi_{s=4}^{2}(0,05)=12.59$, temos que $35.277>12.59$, então rejeitamos a hipótese nula o que significa que os instrumentos têm vícios aditivos e multiplicativos, ou seja, que pelo menos um instrumento tem viés aditivo não nulo ou pelo menos um deles tem vício multiplicativo diferente de um.

Agora, passemos a verificar se esses vícios aditivos e multiplicativos são todos iguais entre eles. Para este caso a hipótese de interesse é dada por:

$$
H_{02}: \alpha_{2}=\alpha_{3}=\alpha_{4}=\alpha, \beta_{2}=\beta_{3}=\beta_{4}=\beta ;
$$

onde a matriz A é dada por:

$$
A=\left(\begin{array}{cccccccccccc}
0 & 1 & -1 & 0 & 0 & 0 & 0 & 0 & 0 & 0 & 0 & 0 \\
0 & 0 & 1 & -1 & 0 & 0 & 0 & 0 & 0 & 0 & 0 & 0 \\
0 & 0 & 0 & 0 & 1 & -1 & 0 & 0 & 0 & 0 & 0 & 0 \\
0 & 0 & 0 & 0 & 0 & 1 & -1 & 0 & 0 & 0 & 0 & 0
\end{array}\right)
$$

de modo que, $A \theta-\mathbf{b}=0$, de onde obtemos:

$$
\begin{gathered}
A \hat{\theta}=\left(\begin{array}{cccc}
0.54631 & -1.8480 & 0.021368 & 0.10320
\end{array}\right)^{\prime} \mathrm{e} \\
{\left[A \hat{I}_{F}^{-1}(\hat{\theta}) A^{\prime}\right]^{-1}=} \\
\left(\begin{array}{llll}
20.409 & 9.8244 & 395.11 & 190.20 \\
9.8244 & 14.693 & 190.20 & 284.46 \\
395.11 & 190.20 & 7830.4 & 3769.5 \\
190.20 & 284.46 & 3769.5 & 5637.6
\end{array}\right) .
\end{gathered}
$$

Neste caso o valor da estatística $\mathrm{W}=23.825$, que indica rejeição de $\mathrm{H}_{0}$, isto é, existe diferencas entre os vícios. 


\section{Instrumentos 2, 4 e 5:}

No caso de testar a hipótese:

$$
H_{01}: \alpha_{2}=\alpha_{3}=\alpha_{4}=0, \beta_{2}=\beta_{3}=\beta_{4}=1 ;
$$

que pode ser escrita como $H_{01}: A \theta=\mathbf{b}$, com a matriz $\mathrm{A}$ e vetor $\mathbf{b}$ dados por:

$$
A=\left(\begin{array}{cccccccccccc}
0 & 1 & 0 & 0 & 0 & 0 & 0 & 0 & 0 & 0 & 0 & 0 \\
0 & 0 & 1 & 0 & 0 & 0 & 0 & 0 & 0 & 0 & 0 & 0 \\
0 & 0 & 0 & 1 & 0 & 0 & 0 & 0 & 0 & 0 & 0 & 0 \\
0 & 0 & 0 & 0 & 1 & 0 & 0 & 0 & 0 & 0 & 0 & 0 \\
0 & 0 & 0 & 0 & 0 & 1 & 0 & 0 & 0 & 0 & 0 & 0 \\
0 & 0 & 0 & 0 & 0 & 0 & 1 & 0 & 0 & 0 & 0 & 0
\end{array}\right) \text { e } \mathbf{b}=\left(\begin{array}{c}
0 \\
0 \\
0 \\
1 \\
1 \\
1
\end{array}\right)
$$

de modo que, temos para cada caso:

$$
A \hat{\theta}-\mathbf{b}=\left(\begin{array}{llllll}
-11.048 & -9.4576 & -7.7428 & 0.61974 & 0.48541 & 0.39167
\end{array}\right)^{\prime},
$$

com:

$$
\begin{aligned}
& {\left[A \hat{I}_{F}^{-1}(\hat{\theta}) A^{\prime}\right]^{-1}=} \\
& \left(\begin{array}{cccccc}
35.197 & -22.885 & -13.224 & 681.42 & -443.06 & -256.01 \\
-22.885 & 24.895 & 0.051169 & -443.06 & 481.97 & 0.99063 \\
-13.224 & 0.051169 & 15.329 & -256.01 & 0.99063 & 296.77 \\
681.42 & -443.06 & -256.01 & 13307 . & -8652.7 & -4999.8 \\
-443.06 & 481.97 & 0.99063 & -8652.7 & 9416.5 & 16.195 \\
-256.01 & 0.99063 & 296.77 & -4999.8 & 16.195 & 5799.6
\end{array}\right),
\end{aligned}
$$

que leva ao valor da estatística $\mathrm{W}=44.208$.

Comparando $\mathrm{W}$ com o valor crítico de qui-quadrado a um nível de significância de $5 \%$ com 6 graus de liberdade, $\chi_{s=4}^{2}(0,05)=12.59$, temos que $44.208>12.59$, então rejeitamos a hipótese nula o que significa que os instrumentos têm vícios aditivos e multiplicativos, ou seja, que pelo menos um instrumento tem viés aditivo não nulo ou pelo menos um deles tem vício multiplicativo diferente de um.

Agora, passemos a verificar se esses vícios aditivos e multiplicativos são todos iguais entre eles. Para este caso a hipótese de interesse é dada por:

$$
H_{02}: \alpha_{2}=\alpha_{3}=\alpha_{4}=\alpha, \beta_{2}=\beta_{3}=\beta_{4}=\beta ;
$$

onde a matriz A é dada por: 


$$
A=\left(\begin{array}{cccccccccccc}
0 & 1 & -1 & 0 & 0 & 0 & 0 & 0 & 0 & 0 & 0 & 0 \\
0 & 0 & 1 & -1 & 0 & 0 & 0 & 0 & 0 & 0 & 0 & 0 \\
0 & 0 & 0 & 0 & 1 & -1 & 0 & 0 & 0 & 0 & 0 & 0 \\
0 & 0 & 0 & 0 & 0 & 1 & -1 & 0 & 0 & 0 & 0 & 0
\end{array}\right)
$$

de modo que, $A \theta-\mathbf{b}=0$, de onde obtemos:

$$
\begin{gathered}
A \hat{\theta}=\left(\begin{array}{lllll}
-1.5906 & -1.7148 & 0.13433 & 0.93739
\end{array}\right)^{\prime} \mathrm{e} \\
{\left[\begin{array}{lllll}
A \hat{I}_{F}^{-1}(\hat{\theta}) A^{\prime} & -1
\end{array}\right]^{-1}=\left(\begin{array}{llll}
34.945 & 12.629 & 676.54 & 244.49 \\
12.629 & 13.922 & 244.49 & 269.53 \\
676.54 & 244.49 & 13212 . & 4774.5 \\
244.49 & 269.53 & 4774.5 & 5267.2
\end{array}\right) .}
\end{gathered}
$$

Neste caso o valor da estatística $\mathrm{W}=41.863$, que indica a rejeição de $\mathrm{H}_{0}$, isto é, existe diferencas entre os vícios.

\section{Instrumentos 3, 4 e 5:}

No caso de testar a hipótese:

$$
H_{01}: \alpha_{2}=\alpha_{3}=\alpha_{4}=0, \beta_{2}=\beta_{3}=\beta_{4}=1 ;
$$

que pode ser escrita como $H_{01}: A \theta=\mathbf{b}$, com a matriz $\mathrm{A}$ e vetor $\mathbf{b}$ dados por:

$$
A=\left(\begin{array}{cccccccccccc}
0 & 1 & 0 & 0 & 0 & 0 & 0 & 0 & 0 & 0 & 0 & 0 \\
0 & 0 & 1 & 0 & 0 & 0 & 0 & 0 & 0 & 0 & 0 & 0 \\
0 & 0 & 0 & 1 & 0 & 0 & 0 & 0 & 0 & 0 & 0 & 0 \\
0 & 0 & 0 & 0 & 1 & 0 & 0 & 0 & 0 & 0 & 0 & 0 \\
0 & 0 & 0 & 0 & 0 & 1 & 0 & 0 & 0 & 0 & 0 & 0 \\
0 & 0 & 0 & 0 & 0 & 0 & 1 & 0 & 0 & 0 & 0 & 0
\end{array}\right) \text { e } \mathbf{b}=\left(\begin{array}{c}
0 \\
0 \\
0 \\
1 \\
1 \\
1
\end{array}\right)
$$

de modo que, temos para cada caso:

$$
A \hat{\theta}-\mathbf{b}=\left(\begin{array}{llllll}
-0.66871 & 0.42590 & 1.6055 & 0.034024 & -0.025098 & -0.091196
\end{array}\right)^{\prime},
$$

com:

$$
\left[A \hat{I}_{F}^{-1}(\hat{\theta}) A^{\prime}\right]^{-1}=\left(\begin{array}{cccccc}
28.982 & -13.834 & -5.5993 & 561.08 & -267.83 & -108.40 \\
-13.834 & 27.687 & -4.3104 & -267.83 & 536.01 & -83.449 \\
-5.5993 & -4.3104 & 14.900 & -108.40 & -83.449 & 288.47 \\
561.08 & -267.83 & -108.40 & 11106 . & -5301.2 & -2146.2 \\
-267.83 & 536.01 & -83.449 & -5301.2 & 10611 . & -1652.5 \\
-108.40 & -83.449 & 288.47 & -2146.2 & -1652.5 & 5712.0
\end{array}\right)
$$


que leva ao valor da estatística $\mathrm{W}=2.1785$

Comparando $\mathrm{W}$ com o valor crítico de qui-quadrado a um nível de significância de $5 \%$ com 6 graus de liberdade, $\chi_{s=4}^{2}(0,05)=12.59$, temos que $2.1785<12.59$, então não rejeitamos a hipótese nula o que significa que os instrumentos não têm vícios aditivos e multiplicativos, ou seja, nenhum instrumento tem viés aditivo não nulo ou vício multiplicativo diferente de um.

Agora, passemos a verificar se esses vícios aditivos e multiplicativos são todos iguais entre eles. Para este caso a hipótese de interesse é dada por:

$$
H_{02}: \alpha_{2}=\alpha_{3}=\alpha_{4}=\alpha, \beta_{2}=\beta_{3}=\beta_{4}=\beta ;
$$

onde a matriz A é dada por:

$$
A=\left(\begin{array}{cccccccccccc}
0 & 1 & -1 & 0 & 0 & 0 & 0 & 0 & 0 & 0 & 0 & 0 \\
0 & 0 & 1 & -1 & 0 & 0 & 0 & 0 & 0 & 0 & 0 & 0 \\
0 & 0 & 0 & 0 & 1 & -1 & 0 & 0 & 0 & 0 & 0 & 0 \\
0 & 0 & 0 & 0 & 0 & 1 & -1 & 0 & 0 & 0 & 0 & 0
\end{array}\right),
$$

de modo que, $A \theta-\mathbf{b}=0$, de onde obtemos:

$$
\begin{aligned}
& A \hat{\theta}=\left(\begin{array}{llll}
-1.0946 & -1.1797 & 0.059122 & 0.066098
\end{array}\right)^{\prime} \mathrm{e} \\
& {\left[A \hat{I}_{F}^{-1}(\hat{\theta}) A^{\prime}\right]^{-1}=\left(\begin{array}{cccc}
25.196 & 7.5781 & 487.79 & 146.71 \\
7.5781 & 13.866 & 146.71 & 268.45 \\
487.79 & 146.71 & 9655.9 & 2904.7 \\
146.71 & 268.45 & 2904.7 & 5315.4
\end{array}\right) .}
\end{aligned}
$$

Neste caso o valor da estatística $\mathrm{W}=2.0389$, que indica não rejeição de $\mathrm{H}_{0}$, isto é, não existe diferencas entre os vícios.

\subsubsection{Hipóteses de interesse} hipóteses:

Para os dados das árvores podemos estar interessados em testar as seguintes

$$
\begin{aligned}
& H_{01}: \alpha_{2}=\alpha_{3}=\alpha_{4}=\alpha_{5}=0, \beta_{2}=\beta_{3}=\beta_{4}=\beta_{5}=1 ; \\
& H_{02}: \alpha_{2}=\alpha_{3}=\alpha_{4}=\alpha_{5}=\alpha, \beta_{2}=\beta_{3}=\beta_{4}=\beta_{5}=\beta
\end{aligned}
$$

Escrevendo as hipóteses $H_{01}$ e $H_{02}$ na forma $H_{0}: A \theta=\mathbf{b}$ vamos utilizar a estatística de Wald mencionada na seção anterior. 
Com o objetivo de testar a precisão dos aparelhos independente da influência pessoal do observador na medição de altura total das árvores, foi feito um ensaio comparativo utilizando 5 aparelhos operados por um único observador e fazendo os testes de TRV e Wald.

Os aparelhos usados foram: o hipsômetro de Blume Leiss, o de Haga, o de Weiss, a Prancheta dendrométrica e a Trena.

Foram medidas 10 árvores utilizando os 5 instrumentos e um único observador, totalizando um total de 50 dados.

\subsubsection{Estimadores de máxima verossimilhança}

Acontece que, para a implementação do teste, necessitamos dos estimadores de máxima verossimilhança, que foram calculados utilizando o programa Ox e o algoritmo EM, obtendo assim os seguintes estimadores:

Tabela 3.8 - Estimativa dos parâmetros para todos os instrumentos para os dados das árvores.

\begin{tabular}{|c|c|c|c|c|c|c|c|}
\hline$\hat{\theta}$ & $\hat{\mu}_{x}$ & $\hat{\alpha}_{2}$ & $\hat{\alpha}_{3}$ & $\hat{\alpha}_{4}$ & $\hat{\alpha}_{5}$ & $\hat{\beta}_{2}$ & $\hat{\beta}_{3}$ \\
& 19.360 & 0.23719 & -0.28999 & 0.65732 & 1.6378 & 1.0368 & 1.0145 \\
\hline$\hat{\beta}_{4}$ & $\hat{\beta}_{5}$ & $\hat{\sigma}_{x}^{2}$ & $\hat{\sigma}_{1}^{2}$ & $\hat{\sigma}_{2}^{2}$ & $\hat{\sigma}_{3}^{2}$ & $\hat{\sigma}_{4}^{2}$ & $\hat{\sigma}_{5}^{2}$ \\
0.96295 & 0.90714 & 8.7847 & 0.18167 & 0.15138 & 0.41184 & 0.32819 & 0.50903 \\
\hline
\end{tabular}

Verificando a tabela acima, é possível verificar que o maior viés aditivo é apresentado pelo instrumento 5, bem como o maior viés multiplicativo.

Obtemos o seguinte resultado para a matriz de informação de Fisher:

$\hat{I}=$

$\begin{array}{rrrrrr}1.1317 & 0.39657 & 0.14262 & 0.16988 & 0.10318 & 7.6775 \\ 0.39657 & 42.199 & -8.5812 & -10.222 & -6.2083 & 816.97 \\ 0.14262 & -8.5812 & 21.195 & -3.6761 & -2.2328 & -166.13 \\ 0.16988 & -10.222 & -3.6761 & 26.091 & -2.6596 & -197.89 \\ 0.10318 & -6.2083 & -2.2328 & -2.6596 & 18.030 & -120.19 \\ 7.6775 & 816.97 & -166.13 & -197.89 & -120.19 & 16186 . \\ 2.7612 & -166.13 & 410.33 & -71.169 & -43.226 & -3290.8 \\ 3.2890 & -197.89 & -71.169 & 505.12 & -51.489 & -3919.9 \\ 1.9976 & -120.19 & -43.226 & -51.489 & 349.06 & -2380.8 \\ 0.00000 & 0.00000 & 0.00000 & 0.00000 & 0.00000 & 0.39427 \\ 0.00000 & 0.00000 & 0.00000 & 0.00000 & 0.00000 & -5.3687 \\ 0.00000 & 0.00000 & 0.00000 & 0.00000 & 0.00000 & 14.701 \\ 0.00000 & 0.00000 & 0.00000 & 0.00000 & 0.00000 & -1.0751 \\ 0.00000 & 0.00000 & 0.00000 & 0.00000 & 0.00000 & -1.5255 \\ 0.00000 & 0.00000 & 0.00000 & 0.00000 & 0.00000 & -0.56274\end{array}$




$\begin{array}{rrrrrr}2.7612 & 3.2890 & 1.9976 & 0.00000 & 0.00000 & 0.00000 \\ -166.13 & -197.89 & -120.19 & 0.00000 & 0.00000 & 0.00000 \\ 410.33 & -71.169 & -43.226 & 0.00000 & 0.00000 & 0.00000 \\ -71.169 & 505.12 & -51.489 & 0.00000 & 0.00000 & 0.00000 \\ -43.226 & -51.489 & 349.06 & 0.00000 & 0.00000 & 0.00000 \\ -3290.8 & -3919.9 & -2380.8 & 0.39427 & -5.3687 & 14.701 \\ 8129.4 & -1409.8 & -856.24 & 0.14180 & -1.9308 & -2.9895 \\ -1409.8 & 10007 . & -1019.9 & 0.16890 & -2.2999 & -3.5609 \\ -856.24 & -1019.9 & 6915.3 & 0.10259 & -1.3969 & -2.1628 \\ 0.14180 & 0.16890 & 0.10259 & 0.064043 & 0.0050786 & 0.0054595 \\ -1.9308 & -2.2999 & -1.3969 & 0.0050786 & 78.539 & 18.386 \\ -2.9895 & -3.5609 & -2.1628 & 0.0054595 & 18.386 & 89.036 \\ 2.6555 & -0.46058 & -0.27974 & 0.0052266 & 2.3780 & 3.6819 \\ -0.54862 & 3.8938 & -0.39691 & 0.0047093 & 3.3741 & 5.2241 \\ -0.20238 & -0.24107 & 1.6343 & 0.0041792 & 1.2447 & 1.9271\end{array}$

\begin{tabular}{|c|c|c|c|c|c|}
\hline 0.00000 & 0.00000 & 0.00000 & & & \\
\hline 0.00000 & 0.00000 & 0.00000 & & & \\
\hline 0.00000 & 0.00000 & 0.00000 & & & \\
\hline 0.00000 & 0.00000 & 0.00000 & & & \\
\hline 0.00000 & 0.00000 & 0.00000 & & & \\
\hline-1.0751 & -1.5255 & -0.56274 & & & \\
\hline 2.6555 & -0.54862 & -0.20238 & & & \\
\hline-0.46058 & 3.8938 & -0.24107 & & & \\
\hline-0.27974 & -0.39691 & 1.6343 & & & \\
\hline 0.0052266 & 0.0047093 & 0.0041792 & & & \\
\hline 2.3780 & 3.3741 & 1.2447 & & & \\
\hline 3.6819 & 5.2241 & 1.9271 & & & \\
\hline 22.461 & 0.67569 & 0.24926 & & & \\
\hline 0.67569 & 34.037 & 0.35366 & & & \\
\hline 0.24926 & 0.35366 & 16.254 & & & \\
\hline \multicolumn{6}{|l|}{$\hat{I}^{-1}=$} \\
\hline 0.89664 & -0.018836 & -0.018430 & -0.017494 & -0.016480 & $6.0412 e-016$ \\
\hline-0.018836 & 1.5380 & 0.84576 & 0.80279 & 0.75635 & -0.077650 \\
\hline-0.018430 & 0.84576 & 2.6387 & 0.78468 & 0.73913 & -0.042699 \\
\hline-0.017494 & 0.80279 & 0.78468 & 2.1894 & 0.70163 & -0.040530 \\
\hline-0.016480 & 0.75635 & 0.73913 & 0.70163 & 2.8988 & -0.038185 \\
\hline $1.1146 \mathrm{e}-017$ & -0.077650 & -0.042699 & -0.040530 & -0.038185 & 0.0040109 \\
\hline$-2.1200 e-018$ & -0.042699 & -0.13320 & -0.039615 & -0.037315 & 0.0022055 \\
\hline$-7.5305 e-019$ & -0.040530 & -0.039615 & -0.11052 & -0.035422 & 0.0020935 \\
\hline
\end{tabular}




\begin{tabular}{|c|c|c|c|c|c|}
\hline $3.1416 \mathrm{e}-018$ & -0.038185 & -0.037315 & -0.035422 & -0.14633 & 0.0019724 \\
\hline$-6.7034 e-017$ & 0.74070 & 0.72153 & 0.68523 & 0.64513 & -0.038260 \\
\hline $1.1949 \mathrm{e}-018$ & -0.010809 & -0.0087770 & -0.0083780 & -0.0077475 & 0.00055832 \\
\hline$-2.2013 e-018$ & 0.011113 & 0.0012951 & 0.0011494 & 0.0013315 & -0.00057400 \\
\hline $1.0318 \mathrm{e}-018$ & -0.00079710 & 0.012964 & 0.00026299 & 0.00033529 & $4.1173 e-005$ \\
\hline $7.9441 \mathrm{e}-019$ & -0.00069121 & 0.00034855 & 0.010329 & 0.00036922 & $3.5703 e-005$ \\
\hline $1.8607 \mathrm{e}-019$ & -0.00063482 & 0.00015407 & 0.00012612 & 0.012658 & $3.2790 \mathrm{e}-005$ \\
\hline $9.0769 e-017$ & $6.4283 e-016$ & $-7.9611 e-016$ & $7.2202 e-015$ & $-5.5364 e-017$ & $8.5156 e-017$ \\
\hline-0.042699 & -0.040530 & -0.038185 & 0.74070 & -0.010809 & 0.011113 \\
\hline-0.13320 & -0.039615 & -0.037315 & 0.72153 & -0.0087770 & 0.0012951 \\
\hline-0.039615 & -0.11052 & -0.035422 & 0.68523 & -0.0083780 & 0.0011494 \\
\hline-0.037315 & -0.035422 & -0.14633 & 0.64513 & -0.0077475 & 0.0013315 \\
\hline 0.0022055 & 0.0020935 & 0.0019724 & -0.038260 & 0.00055832 & -0.00057400 \\
\hline 0.0068804 & 0.0020462 & 0.0019274 & -0.037269 & 0.00045336 & $-6.6894 \mathrm{e}-005$ \\
\hline 0.0020462 & 0.0057089 & 0.0018296 & -0.035394 & 0.00043275 & $-5.9369 e-005$ \\
\hline 0.0019274 & 0.0018296 & 0.0075583 & -0.033323 & 0.00040018 & $-6.8776 e-005$ \\
\hline-0.037269 & -0.035394 & -0.033323 & 16.080 & -0.0068729 & 0.0034627 \\
\hline 0.00045336 & 0.00043275 & 0.00040018 & -0.0068729 & 0.013521 & -0.0027338 \\
\hline$-6.6894 e-005$ & $-5.9369 e-005$ & $-6.8776 e-005$ & 0.0034627 & -0.0027338 & 0.012069 \\
\hline-0.00066964 & $-1.3584 e-005$ & $-1.7319 e-005$ & -0.0020952 & -0.00095991 & -0.0016515 \\
\hline$-1.8003 e-005$ & -0.00053350 & $-1.9071 e-005$ & -0.00065778 & -0.00090611 & -0.0015577 \\
\hline$-7.9581 e-006$ & $-6.5146 e-006$ & -0.00065380 & -0.0029355 & -0.00068391 & -0.0011780 \\
\hline$-1.6551 e-017$ & $-5.5932 e-017$ & $-2.5368 e-017$ & & & \\
\hline-0.0 & -0.00069121 & -0.00 & & & \\
\hline 0.012964 & 0.00034855 & 0.00015407 & & & \\
\hline 0.00026299 & 0.010329 & 0.00012612 & & & \\
\hline 0.00033529 & 0.00036922 & 0.012658 & & & \\
\hline $4.1173 e-005$ & $3.5703 e-005$ & $3.2790 \mathrm{e}-005$ & & & \\
\hline-0.00066964 & $-1.8003 e-005$ & $-7.9581 e-006$ & & & \\
\hline$-1.3584 e-005$ & -0.00053350 & $-6.5146 e-006$ & & & \\
\hline$-1.7319 e-005$ & $-1.9071 \mathrm{e}-005$ & -0.00065380 & & & \\
\hline-0.0020952 & -0.00065778 & -0.0029355 & & & \\
\hline-0.00095991 & -0.00090611 & -0.00068391 & & & \\
\hline-0.0016515 & -0.0015577 & -0.0011780 & & & \\
\hline 0.044995 & -0.00054760 & -0.00041363 & & & \\
\hline-0.00054760 & 0.029785 & -0.00039045 & & & \\
\hline-0.00041363 & -0.00039045 & 0.061799 & & & \\
\hline
\end{tabular}

No caso de testar a hipótese:

$$
H_{01}: \alpha_{2}=\alpha_{3}=\alpha_{4}=\alpha_{5}=0, \beta_{2}=\beta_{3}=\beta_{4}=\beta_{5}=1 ;
$$


que pode ser escrita como $H_{01}: A \theta=\mathbf{b}$, com a matriz $\mathrm{A}$ e vetor $\mathbf{b}$ dados por:

$$
A=\left(\begin{array}{lllllllllllllll}
0 & 1 & 0 & 0 & 0 & 0 & 0 & 0 & 0 & 0 & 0 & 0 & 0 & 0 & 0 \\
0 & 0 & 1 & 0 & 0 & 0 & 0 & 0 & 0 & 0 & 0 & 0 & 0 & 0 & 0 \\
0 & 0 & 0 & 1 & 0 & 0 & 0 & 0 & 0 & 0 & 0 & 0 & 0 & 0 & 0 \\
0 & 0 & 0 & 0 & 1 & 0 & 0 & 0 & 0 & 0 & 0 & 0 & 0 & 0 & 0 \\
0 & 0 & 0 & 0 & 0 & 1 & 0 & 0 & 0 & 0 & 0 & 0 & 0 & 0 & 0 \\
0 & 0 & 0 & 0 & 0 & 0 & 1 & 0 & 0 & 0 & 0 & 0 & 0 & 0 & 0 \\
0 & 0 & 0 & 0 & 0 & 0 & 0 & 1 & 0 & 0 & 0 & 0 & 0 & 0 & 0 \\
0 & 0 & 0 & 0 & 0 & 0 & 0 & 0 & 1 & 0 & 0 & 0 & 0 & 0 & 0
\end{array}\right) \text { e } \mathbf{b}=\left(\begin{array}{c}
0 \\
0 \\
0 \\
0 \\
1 \\
1 \\
1 \\
1
\end{array}\right)
$$

de modo que:

$A \hat{\theta}-\mathbf{b}=\left(\begin{array}{llllllll}0.23719 & -0.28999 & 0.65732 & 1.6378 & 0.036819 & 0.014462 & -0.037052 & -0.092861\end{array}\right)^{\prime}$

com:

$$
\begin{gathered}
{\left[A \hat{I}_{F}^{-1}(\hat{\theta}) A^{\prime}\right]^{-1}=} \\
\left(\begin{array}{cccccccc}
42.060 & -8.6312 & -10.281 & -6.2444 & 814.28 & -167.10 & -199.04 & -120.89 \\
-8.6312 & 21.177 & -3.6975 & -2.2458 & -167.10 & 409.99 & -71.584 & -43.478 \\
-10.281 & -3.6975 & 26.066 & -2.6751 & -199.04 & -71.584 & 504.63 & -51.789 \\
-6.2444 & -2.2458 & -2.6751 & 18.020 & -120.89 & -43.478 & -51.789 & 348.87 \\
814.28 & -167.10 & -199.04 & -120.89 & 16128 . & -3309.9 & -3942.6 & -2394.7 \\
-167.10 & 409.99 & -71.584 & -43.478 & -3309.9 & 8121.8 & -1418.2 & -861.40 \\
-199.04 & -71.584 & 504.63 & -51.789 & -3942.6 & -1418.2 & 9996.6 & -1026.1 \\
-120.89 & -43.478 & -51.789 & 348.87 & -2394.7 & -861.40 & -1026.1 & 6911.3
\end{array}\right)
\end{gathered}
$$

que leva ao valor da estatística $\mathrm{W}=44.346$.

Comparando $\mathrm{W}$ com o valor crítico de qui-quadrado a um nível de significância de $1 \%$ com 8 graus de liberdade, $\chi_{s=8}^{2}(0,01)=20.0902$, temos que $44.346>$ 20.0902, então rejeitamos a hipótese nula o que significa que os instrumentos têm vícios aditivos e multiplicativos, ou seja, que pelo menos um instrumento tem viés aditivo não nulo ou pelo menos um deles tem vício multiplicativo diferente de um.

Agora, passemos a verificar se esses vícios aditivos e multiplicativos são todos iguais entre eles. Para este caso a hipótese de interesse é dada por:

$$
H_{02}: \alpha_{2}=\alpha_{3}=\alpha_{4}=\alpha_{5}=\alpha, \beta_{2}=\beta_{3}=\beta_{4}=\beta_{5}=\beta ;
$$

onde a matriz A é dada por: 


$$
A=\left(\begin{array}{ccccccccccccccc}
0 & 1 & -1 & 0 & 0 & 0 & 0 & 0 & 0 & 0 & 0 & 0 & 0 & 0 & 0 \\
0 & 0 & 1 & -1 & 0 & 0 & 0 & 0 & 0 & 0 & 0 & 0 & 0 & 0 & 0 \\
0 & 0 & 0 & 1 & -1 & 0 & 0 & 0 & 0 & 0 & 0 & 0 & 0 & 0 & 0 \\
0 & 0 & 0 & 0 & 0 & 1 & -1 & 0 & 0 & 0 & 0 & 0 & 0 & 0 & 0 \\
0 & 0 & 0 & 0 & 0 & 0 & 1 & -1 & 0 & 0 & 0 & 0 & 0 & 0 & 0 \\
0 & 0 & 0 & 0 & 0 & 0 & 0 & 1 & -1 & 0 & 0 & 0 & 0 & 0 & 0
\end{array}\right)
$$

de modo que, $A \theta-\mathbf{b}=0$, de onde obtemos:

$$
\begin{gathered}
A \hat{\theta}=\left(\begin{array}{llllllll}
0.52718 & -0.94731 & -0.98046 & 0.022357 & 0.051314 & 0.055809
\end{array}\right)^{\prime} \mathrm{e} \\
{\left[A \hat{I}_{F}^{-1}(\hat{\theta}) A^{\prime}\right]^{-1}=\left(\begin{array}{lllllll}
34.867 & 23.439 & 9.1578 & 675.20 & 453.78 & 177.29 \\
23.439 & 32.083 & 12.542 & 453.78 & 621.12 & 242.80 \\
9.1578 & 12.542 & 16.839 & 177.29 & 242.80 & 326.00 \\
675.20 & 453.78 & 177.29 & 13374 . & 8988.3 & 3511.9 \\
453.78 & 621.12 & 242.80 & 8988.3 & 12304 . & 4809.1 \\
177.29 & 242.80 & 326.00 & 3511.9 & 4809.1 & 6458.1
\end{array}\right) .}
\end{gathered}
$$

Neste caso o valor da estatística e $\mathrm{W}=39.166$, que é maior que $\chi_{6}^{2}(0,05)=12,59,0$ que indica rejeição de $\mathrm{H}_{0}$, isto é, existe diferencas entre os vícios.

Resumindo temos a seguinte tabela:

Tabela 3.9 - Teste de Wald

\begin{tabular}{|c|c|c|c|c|}
\hline instrumentos & $\mathrm{H}_{01}$ & nível descritivo & $\mathrm{H}_{02}$ & nível descritivo \\
\hline 2 e 3 & 31.407 & 0.000001 & 12.841 & 0.000814 \\
2 e 4 & 4280.4 & 0.000000 & 22.809 & 0.000006 \\
2 e 5 & 35.634 & 0.000000 & 19.448 & 0.000030 \\
3 e 4 & 1.3254 & 0.170797 & 1.1710 & 0.212642 \\
3 e 5 & 2.2277 & 0.182834 & 1.0479 & 0.296088 \\
4 e 5 & 1.3361 & 0.171254 & 0.25703 & 0.439700 \\
2,3 e 4 & 38.339 & 0.000004 & 31.184 & 0.000001 \\
2,3 e 5 & 35.277 & 0.0000017 & 23.825 & 0.000040 \\
2,4 e 5 & 44.208 & 0.0000 & 41.863 & 0.0000 \\
3,4 e 5 & 2.1785 & 0.0998021 & 2.0389 & 0.183905 \\
$2,3,4$ e 5 & 44.346 & 0.0000002 & 39.166 & 0.000003 \\
\hline
\end{tabular}

Observando a tabela acima, foi possível verificar que para os dados das árvores foi observado que os instrumentos 3, 4 e 5 (Haga, Weise e Prancheta dendrométrica) possuem os mesmos viéses aditivos e multiplicativos, que por sua vez, esse viés é diferente do viés do instrumento 2 (Blume-Leiss). 


\subsection{Teste da razão de verossimilhanças}

O teste foi feito utilizando os mesmos dados utilizados no teste de wald e consideramos as mesmas hipóteses mencionadas na seção 3.1 deste trabalho e obtemos os seguintes resultados para as 10 árvores e as dez combinações de 3 instrumentos, combinações de quatro a quatro instrumentos e por fim para os 5 instrumentos:

Nota-se na tabela 3.10 que os viéses aditivos e multiplicativos ao comparar entre os instrumentos 3, 4 e 5 não diferem entre si. Contudo, esses mesmos viéses diferem ao comparar qualquer um ou mais desses instrumentos com o 2 (Brume-Leiss).

Tabela 3.10 - Teste da razão da Verossimilhança

\begin{tabular}{|c|c|c|c|c|}
\hline instrumentos & $\mathrm{H}_{01}$ & nível de sig. & $\mathrm{H}_{02}$ & nível de sig. \\
\hline 2 e 3 & 8.5268 & 0.030003 & 7.4412 & 0.012109 \\
2 e 4 & 9.1925 & 0.023188 & 8.3820 & 0.007565 \\
2 e 5 & 8.8166 & 0.026837 & 7.9780 & 0.009259 \\
3 e 4 & 3.6779 & 0.146181 & 3.4312 & 0.089928 \\
3 e 5 & 4.6171 & 0.11474 & 3.2149 & 0.1002 \\
4 e 5 & 3.5328 & 0.150978 & 0.4780 & 0.393701 \\
2,3 e 4 & $\mathrm{~N} / \mathrm{C}$ & $\mathrm{N} / \mathrm{C}$ & $\mathrm{N} / \mathrm{C}$ & $\mathrm{N} / \mathrm{C}$ \\
2,3 e 5 & 8.8120 & 0.0592285 & 8.5639 & 0.02 \\
2,4 e 5 & $\mathrm{~N} / \mathrm{C}$ & $\mathrm{N} / \mathrm{C}$ & $\mathrm{N} / \mathrm{C}$ & $\mathrm{N} / \mathrm{C}$ \\
3,4 e 5 & $\mathrm{~N} / \mathrm{C}$ & $\mathrm{N} / \mathrm{C}$ & $\mathrm{N} / \mathrm{C}$ & $\mathrm{N} / \mathrm{C}$ \\
$2,3,4$ e 5 & $\mathrm{~N} / \mathrm{C}$ & $\mathrm{N} / \mathrm{C}$ & $\mathrm{N} / \mathrm{C}$ & $\mathrm{N} / \mathrm{C}$ \\
\hline
\end{tabular}

É importante salientar que foram rejeitadas as hipóteses nulas para todos os casos em que o nível de significância foi menor que $5 \%$.

Também, para este teste é importante observar o seguinte:

Os casos indicados por N/C foram decorridos por problema de precisão do software ox no cálculo de certos determinantes quando seus valores ficam bem próximos de zero (por volta de $10^{-15}$ ) não foram calculados corretamente.

Nota-se também na tabela acima que ao aumentar a quantidade de três instrumentos para quatro, nota-se um aumento no valor do teste de razão de verossimilhança nas duas hipóteses de interesse.

\subsection{Resultados}

De forma geral, para os dados das árvores foi observado que os instrumentos 3, 4 e 5 (Haga, Weise e Prancheta dendrométrica) possuem os mesmos viéses aditivos e multiplicativos, que por sua vez, esse viés é diferente do viés do instrumento 2 (BlumeLeiss). 


\section{Capítulo 4}

\section{Conclusões}

\subsection{Dados reais}

Para dados reais, comparando os resultados obtidos com a utilização do teste de Wald com o teste da razão de verossimilhanças é possível verificar que eles são na maioria das vezes equivalentes.

Após o cálculo, utilizando combinações de três a três, quatro a quatro e utilizando todos os instrumentos com os dados das árvores, foi possível observar o seguinte:

- Problemas quanto a precisão no cálculo de certos determinantes, principalmente quando os valores estão bem próximos de zero (por volta de $10^{-15}$ ) acarretando problemas no cálculo dos testes, principalmente no cálculo da estatística da razão da verossimilhanças, com o programa acusando NaN (não foi possível calcular no $\mathrm{Ox})$

- Em outros casos mais raros a estatística da razão é negativa, o que gerou problemas para a comparação entre os testes de razão de verossimilhanças e Wald para as duas hipóteses de interesse.

- Quando não se verificam os problemas discutidos anteriormente, nota-se valores bem próximos para os dois testes.

Contudo, mesmo para dados reais, o teste da razão pode apresentar dificuldades de cálculo, especialmente em situações onde a matriz $\hat{\Sigma}$ tem determinante bem próximo de zero. Isso foi notado várias vezes no caso dos dados das árvores.

\subsection{Dados simulados}

Quando observamos a tabela 4.1 que contém os dados que foram gerados, notamos o seguinte:

1 - Quando aumentamos o tamanho da amostra notamos: 
- diminuição na proporção de rejeições da hipótese nula em ambas as hipóteses de interesse no teste de Wald.

- estabilidade na proporção de rejeições para o teste de razão de verossimilhanças, fazendo com que ele independa do tamanho da amostra e também da quantidade de simulações.

- diminuição na quantidade de “missings" em ambos os testes (Wald e razão de verossimilhança)s.

- diminuição na quantidade de valores negativos (representados como "neg" em ambos os testes).

2 - Quando aumentamos a quantidade de simulações notamos:

- diminuição na proporção de rejeições no teste de Wald.

- estabilidade na proporção de rejeições no teste de razão de verossimilhanças.

- estabilidade na quantidade de "missings" e de "neg", ao fixar o tamanho da amostra e aumentar apenas a quantidade de simulações em todos os testes e hipóteses de interesse.

\subsection{De forma geral}

Para ambos os testes obsevamos o seguinte:

- Maior estabilidade do teste de Wald em relação ao teste da razão da verossimilhanças, ocorrendo uma menor incidência de problemas computacionais durante o cálculo.

- Foram tentadas simulações para o caso $\mathrm{p}=4$ instrumentos, mas aconteceu que a proporção de rejeições era muito alta e também a quantidade de "miss"e de "neg" é muito elevada, e sendo assim, essa parte é inviável para os objetivos do nosso trabalho.

Para a continuidade desse trabalho pretendemos estudar o comportamento desses testes através de simulações para diversos modelos de calibração comparativa e estudos sobre o poder dos testes de interesse. 


\section{Apêndice A}

\section{Resultados importantes}

Resultado A.1 Se A é uma matriz $p \times p$ e $\mathbf{x}$ é um vetor $p \times 1$, então:

$$
\mathbf{x}^{\prime} \mathbf{A} \mathbf{x}=\operatorname{tr}\left(\mathbf{x}^{\prime} \mathbf{A} \mathbf{x}\right)=\operatorname{tr}\left(\mathbf{A} \mathbf{x} \mathbf{x}^{\prime}\right) .
$$

Resultado A.2 Seja C uma matriz inversível.

Se $\mathbf{A}=\mathbf{C}+\lambda \mathbf{b b}^{\prime}$, então

$$
|A|=|C|\left(1+C^{\prime} C^{-1} b\right) \text { e } A^{-1}=C^{-1}-\frac{\lambda C^{-1} b b^{\prime} C^{-1}}{1+b^{\prime} C^{-1} b .}
$$

Mais detalhes são encontrados em Rao (1973).

Resultado A.3 DETERMINANTE DE UM PRODUTO - se A e B são matrizes quadradas de ordem $n$, então,

$$
|A B|=|A||B| .
$$

Demonstração, vide Ayres (1976)

Consequência de (A.3): Se A é uma matriz quadrada de dimensão $n \times n$, temos que $\operatorname{det}\left(\mathrm{A}^{-1}\right)=\frac{1}{|A|}$.

Resultado A.4 DERIVADA DE UM DETERMINANTE - Seja A uma matriz quadrada de ordem $n$, cujos elementos são funções diferenciáveis de uma variável $x$.

Então, a derivada, $\frac{d}{d x}|A|$, de $|A|$ em relação a $x$, é a soma de $n$ determinantes obtidos substituindo os elementos da primeira linha (ou coluna) de $|A|$ por suas derivadas em relação a $x$. 
Demonstração, vide Ayres (1976).

Resultado A.5 Seja A uma matriz diagonal, então essa matriz será inversível e terá como resultado uma outra matriz $A^{-1}$ diagonal cujos elementos dessa diagonal terão valores inversos aos dos respectivos elementos da matriz diagonal A, ou seja, se

$$
A=\left(\begin{array}{cccc}
\sigma_{1}^{2} & 0 & \cdots & 0 \\
0 & \sigma_{2}^{2} & \cdots & 0 \\
\vdots & \vdots & \ddots & \vdots \\
0 & 0 & \cdots & \sigma_{p}^{2}
\end{array}\right) \Rightarrow A^{-1}=\left(\begin{array}{cccc}
\frac{1}{\sigma_{1}^{2}} & 0 & \cdots & 0 \\
0 & \frac{1}{\sigma_{2}^{2}} & \cdots & 0 \\
\vdots & \vdots & \ddots & \vdots \\
0 & 0 & \cdots & \frac{1}{\sigma_{p}^{2}}
\end{array}\right) .
$$

Resultado A.6 Se A e uma matriz que pode ser particionada, de modo que:

$$
A=\left(\begin{array}{ll}
A_{11} & A_{12} \\
A_{21} & A_{22}
\end{array}\right) .
$$

Então,

$$
|A|=\left|A_{22}\right|\left|A_{11}-A_{12} A_{22}^{-1} A_{21}\right|=\left|A_{11}\right| \cdot\left|A_{22}-A_{21} A_{11}^{-1} A_{12}\right|,
$$

$\mathrm{e}$

$$
A=\left[\begin{array}{ll}
A_{11} & A_{12} \\
A_{21} & A_{22}
\end{array}\right]^{-1}=\left[\begin{array}{ll}
A^{11} & A^{12} \\
A^{21} & A^{22}
\end{array}\right] .
$$

onde:

$$
\begin{gathered}
A^{11}=\left(A_{11}-A_{12} A_{22}^{-1} A_{21}\right)^{-1}, \quad A^{12}=-A_{11}^{-1} A_{12} A^{22}, \\
A_{21}=-A^{22} A_{21} A_{11}^{-1} \quad A^{22}=\left(A_{22}-A_{21} A_{11}^{-1} A_{12}\right)^{-1} .
\end{gathered}
$$

Resultado A.7 - Sejam $a$ e $b$ constantes reais, $I_{n}$ matriz identidade e $J_{n}$ matriz com todos os elementos iguais a 1, então,

$$
\begin{aligned}
& a I_{n}+b J_{n}^{-1}=\frac{1}{a}\left(I_{n}-\frac{b}{a+b} J_{n}\right), \\
& \left|a I_{n}+b J_{n}\right|=a^{n-1}(a+n b), a \neq 0, a \neq n b .
\end{aligned}
$$

Resultado A.8 Em termos de derivadas parciais podemos destacar os seguintes resultados Kaplan (1985):

- Seja $Z=f(x, y)$ uma função definida num domínio D do plano $x y$ e seja $\left(x_{1}, y_{1}\right)$ um ponto de D. Então, a função $f\left(x_{1}, y_{1}\right)$ depende unicamente de $x$ e é definida num intervalo que contém $x_{1}$. Logo é possível que exista a sua derivada em relação 
a $x$, no ponto $x=x_{1}$. Se essa derivada existir, seu valor será chamado de derivada parcial de $f(x, y)$ em relação a $x$ no ponto $\left(x_{1}, y_{1}\right)$, e ela é designada por $\frac{\partial f}{\partial x}\left(x_{1}, y_{1}\right)$ ou por $\frac{\partial z}{\partial x}\left(x_{1}, y_{1}\right)$,

Defini-se analogamente a derivada parcial $\frac{\partial z}{\partial y}\left(x_{1}, y_{1}\right)$; desta vez, fixamos $x$, que igualmente a $x_{1}$, e derivamos em relação a $y$. Com isso obtemos:

$$
\frac{\partial f}{\partial y}\left(x_{1}, y_{1}\right)=\frac{\partial z}{\partial y} \text { em relação ao ponto }\left(x_{1}, y_{1}\right),
$$

- Derivada de uma função com respeito a um vetor: Seja $f$ uma função de $n$ variáveis reais independentes $x_{1}, x_{2}, \cdots, x_{k}$. A derivada da função $f$ com respeito ao vetor $X$, onde $X=\left(\begin{array}{llll}x_{1} & x_{2} & \cdots & x_{k}\end{array}\right)^{\prime}$ é denotado por $\frac{\partial f}{\partial x}$ e é definido por: $\frac{\partial f}{\partial x}=\left(\begin{array}{llll}\frac{\partial f}{\partial x_{1}} & \frac{\partial f}{\partial x_{2}} & \cdots & \frac{\partial f}{\partial x_{k}}\end{array}\right)^{\prime}$,

- Seja $\ell$ uma função linear de $k$ variáveis reais independentes definidas por $\ell(x)=$ $\sum_{i=1}^{k} a_{i} x_{i}=a^{\prime} x=x^{\prime} a$, onde $a=\left(\begin{array}{llll}a_{1} & a_{2} & \cdots & a_{k}\end{array}\right)^{\prime}$ e os $a_{i}$ são constantes. Então $\frac{\partial \ell}{\partial x}=a$,

- Seja $q$ uma forma quadrática de $k$ variáveis reais independentes $x_{1}, x_{2}, \cdots, x_{k}$ definida por $q(x)=x^{\prime} A x$, onde $A=\left[a_{i j}\right]$ é uma matriz simétrica $k \times k$ de constantes.

$$
\text { Então } \frac{\partial q}{\partial x}=2 A x \text {, }
$$

- Derivada de uma função com respeito a uma matriz. Seja $f$ uma função de uma matriz $X$ de dimensão $m \times n$ definida por:

$$
X=\left[\begin{array}{cccc}
x_{11} & x_{12} & \cdots & x_{1 n} \\
x_{21} & x_{22} & \cdots & x_{2 n} \\
\vdots & \vdots & & \vdots \\
x_{m 1} & x_{m 2} & \cdots & x_{m n}
\end{array}\right] \text { e assume que cada derivada parcial } \frac{\partial f}{\partial x_{i j}} \text { existe. Então }
$$

a derivada de $f$ com respeito a matriz $X$ é denotada por $\frac{\partial f}{\partial X}$ e definida por $\left[\frac{\partial f}{\partial X_{i j}}\right]$.

- Seja $a$ um vetor $k \times 1$ de constantes e seja $X$ uma matriz simétrica $k \times k$ de variáveis reais independentes e define uma função $u$ por: $u(x)=a^{\prime} x a$; então $\frac{\partial u}{\partial x}=2 a a^{\prime}-D_{a a^{\prime}}$, onde $D_{a a^{\prime}}$ é definida como uma matriz diagonal $k \times k$ do qual o i-ésimo elemento dessa diagonal é igual a diagonal do elemento da matriz $a a^{\prime}$.,

- Seja $X$ uma matriz simétrica não singular $k \times k$ de variáveis reais independentes, então $\frac{\partial(\log |X|)}{\partial X}=2 X^{-1}-D_{X^{-1}}$, onde $D_{X^{-1}}$ é uma matriz diagonal com o i-ésimo elemento da diagonal é igual ao i-ésimo elemento da diagonal $X^{-1}$, 
- Seja $X$ uma matriz $k \times k$ de variáveis reais independentes; então $\frac{\partial[\operatorname{tr}(X)]}{\partial X}=I$.

Resultado A.9 Seja $f_{x}(x / \theta)=h(x)=h(x) \exp \sum_{i=1}^{p} T_{i}(x) c_{i}(\theta)+d(\theta)$ para todo $\theta \epsilon \Theta$ e todo x pertencente ao suporte da distribuicão de $\mathrm{X}$ e seja $\mathrm{C}$ o interior do conjunto onde $\left(c_{1}(\theta), c_{2}(\theta), \cdots, c_{p}(\theta)\right)^{\prime}$ está definido para todo $\theta \in \Theta \subseteq \Re^{p}$. Se as equações

$$
\mathbb{E}_{\theta}\left(T_{i}(X)\right)=T_{i}(X)
$$

para $i=1,2, \cdots, p$, tem solução $\hat{\theta}(X)=\left(\hat{\theta}_{1}(X), \hat{\theta}_{2}(X), \cdots, \hat{\theta}_{p}(X)\right)^{\prime}$, para o qual $\hat{\theta}(X)=\left(c_{1} \hat{\theta}(X), c_{2} \hat{\theta}(X), \cdots, c_{p} \hat{\theta}(X)\right)^{\prime} \epsilon C$, então $\hat{\theta}(X)$ é o único estimador de máxima verossimilhança do parâmetro $\theta$.

Demostração: Veja Bickel e Doksun (1976).

Resultado A.10 Seja $y_{k}=\left(y_{1 k}, y_{2 k}, \cdots, y_{p k}\right)^{\prime}$ com distribuição normal multivariada, com vetor de médias $\mu_{t}=\left(\mu_{1}, \mu_{2}, \cdots, \mu_{p}\right)^{\prime}$ e matriz de covariância $\Sigma$, onde os elementos de $\Sigma$ são do tipo $\sigma_{i j}$. Seja uma amostra aleatória de vetores independentes $Y_{k}, k=1, \cdots, n$, onde:

$$
\bar{Y}_{i}=\frac{1}{n} \sum_{k=1}^{n} y_{i k}
$$

e $S_{i j}=\frac{1}{n} \sum_{k=1}^{n}\left(y_{i k}-\bar{Y}_{i}\right)\left(y_{j k}-\bar{Y}_{j}\right)$, com $i, j=1, \cdots, p$. Então,

- $\mathbb{E}\left(\bar{Y}_{i} \bar{Y}_{y}\right)=\frac{1}{n} \sigma_{i j}+\mu_{i} \mu_{j}$

- $\operatorname{Cov}\left(S_{i j}, S_{k m}\right)=\frac{1}{n}\left(\sigma_{i k} \sigma_{j m}+\sigma_{i m} \sigma_{j k}\right)$,

- $\operatorname{Var}\left(S_{i j}\right)=\frac{1}{n}\left(\sigma_{i i} \sigma_{i j}+\sigma_{i j}^{2}\right)$.

Prova: Veja Fuller (1987).

Resultado A.11 Principais propriedades de somatórios e produtórios.

$$
\begin{aligned}
& \text { - } \sum_{i=k}^{n} \alpha a_{i}=\alpha \sum_{i=k}^{n} a_{i}, \\
& \text { - } \sum_{i=k}^{n} \alpha=(n-k+1) \alpha,
\end{aligned}
$$


- $\sum_{i=k}^{n}\left(a_{i}+b_{i}\right)=\sum_{i=k}^{n} a_{i}+\sum_{i=k}^{n} b_{i}$,

- $\sum_{i, j=1}^{n} a_{i j}=\sum_{j=1}^{n} \sum_{i=1}^{n} a_{i j}$

- $\sum_{i=1}^{n}\left(\sum_{j=1}^{n} a_{i j}\right)=\sum_{j=1}^{n}\left(\sum_{i=1}^{n} a_{i j}\right)$,

- $\sum_{i=1}^{n}\left(\sum_{j=1}^{n} a_{i} b_{j}\right)=\left(\sum_{i=1}^{n} a_{i}\right)\left(\sum_{j=1}^{n} b_{j}\right)$,

- $\sum_{i=1}^{n}\left(\sum_{j=1}^{m} a_{i} b_{j}\right)=\sum_{j=1}^{m}\left(\sum_{i=1}^{n} a_{i} b_{j}\right)$,

- $\left(\sum_{i=1}^{n} a_{i}\right)^{2}=\sum_{i=1}^{n} a_{i}^{2}+2 \sum_{1 \leq i \leq j \leq n}^{n} a_{i} b_{i}$

- $\prod_{i=1}^{n} k a_{i}=k^{n} \prod_{i=1}^{n} a_{i}$

- $\prod_{i=k}^{n} \alpha=\alpha^{n-k+1}$

- $\prod_{i=k}^{n} a_{i} b_{i}=\prod_{i=k}^{n} a_{i} \prod_{i=k}^{n} b_{i}$

- $\prod_{i, j=1}^{n} a_{i j}=\prod_{j=1}^{n} \prod_{i=1}^{n} a_{i j}$

- $\prod_{i=1}^{n}\left(\prod_{j=1}^{n} a_{i j}\right)=\prod_{j=1}^{n}\left(\prod_{i=1}^{n} a_{i j}\right)$

- $\prod_{i=1}^{n}\left(\prod_{j=1}^{m} a_{i} b_{j}\right)=\prod_{j=1}^{m}\left(\prod_{i=1}^{n} a_{i} b_{j}\right)$.

Mais detalhes em Neter et al (1994) e Neter, Wasserman e Whitemore (1993).

Resultado A.12 Seja A um vetor linha dimensão $1 \times p, \Sigma$ uma matriz dimensão $p \times p$ e B um vetor coluna dimensão $p \times 1$.

Então: 


$$
A \Sigma B=B^{t} \Sigma A^{t} .
$$

Mais detalhes em Graybill, (1983).

obs.

- $|A|$ significa determinante da matriz A.

- Os resultados descritos neste apêndice, foram utilizados nos apêndices B, C e D. 


\section{Apêndice B}

\section{Função de verossimilhança para os dados observados}

Obs.: Para este apêndice e o próximo, o símbolo "'" significa derivada e o símbolo " $t$ " como expoente significa matriz transposta.

Assim, para os dados observados a função de verossimilhança pode ser escrita a partir da função distribuição normal p-variada, com função densidade dada por:

$$
f(\mathbf{Y})=\frac{1}{2 \pi^{\frac{p}{2}}|\Sigma|^{\frac{1}{2}}} e^{-\frac{1}{2}(\mathbf{Y}-\mu)^{\prime} \Sigma^{-1}(\mathbf{Y}-\mu)}
$$

com vetor de médias e matriz de covariâncias dada, respectivamente por:

$$
\mu=\left(\begin{array}{c}
\alpha_{1}+\beta_{1} \mu_{x} \\
\vdots \\
\alpha_{p}+\beta_{p} \mu_{x}
\end{array}\right) \text { e } \Sigma=\sigma_{x}^{2} \beta \beta^{\prime}+\Psi
$$

onde $\psi=\operatorname{diag}\left(\sigma_{1}^{2}, \cdots, \sigma_{p}^{2}\right)$.

Assim, para o caso dos dados observados obtemos o seguinte logarítmo da função de verossimilhança:

$$
\begin{aligned}
& \ln \left(L\left(\theta \mid y_{1}, \cdots, y_{n}\right)\right)=-n \ln \left((2 \pi)^{\frac{p}{2}}|\Sigma|^{\frac{1}{2}}\right)-\frac{1}{2} \sum_{j=1}^{n}\left(\mathbf{Y}_{j}-\mu\right)^{\prime} \Sigma^{-1}\left(\mathbf{Y}_{j}-\mu\right) \\
= & -n\left[\frac{p}{2} \ln (2 \pi)+\frac{1}{2} \ln \left|\sigma_{x}^{2} \beta \beta+\psi\right|\right]-\frac{1}{2} \sum_{j=1}^{n}\left[\operatorname{tr} \Sigma^{-1}\left(\mathbf{Y}_{j}-\mu\right)\left(\mathbf{Y}_{j}-\mu\right)^{\prime} \cdot\right]
\end{aligned}
$$


Seja $I=\frac{1}{2} \ln \left|\sigma_{x}^{2} \beta \beta+\psi\right|, I I=\Sigma^{-1}$ e $I I I=\left(\mathbf{Y}_{i}-\mu\right)\left(\mathbf{Y}_{i}-\mu\right)^{\prime}$.

Temos:

$I=\frac{1}{2} \ln \left|\sigma_{x}^{2} \beta \beta+\psi\right|, I=\frac{1}{2} \ln |\psi|\left|\left(\sigma_{x}^{2} \beta^{\prime} \psi^{-1} \beta+1\right)\right|$, ou seja,

$=\frac{1}{2} \ln \left(\prod_{i=1}^{p} \sigma_{i}^{2}\right) \mid\left(\sigma_{x}^{2}\left(\begin{array}{llll}\beta_{1} & \beta_{2} & \cdots & \beta_{p}\end{array}\right)\left(\begin{array}{cccc}\frac{1}{\sigma_{1}^{2}} & 0 & \cdots & 0 \\ 0 & \frac{1}{\sigma_{2}^{2}} & \cdots & 0 \\ \vdots & \vdots & \ddots & \vdots \\ 0 & 0 & \cdots & \frac{1}{\sigma_{p}^{2}}\end{array}\right)\left(\begin{array}{c}\beta_{1} \\ \beta_{2} \\ \vdots \\ \beta_{p}\end{array}\right)+1\right)$,

$=\frac{1}{2} \ln \left(\prod_{i=1}^{p} \sigma_{i}^{2}\right)\left|\left(\sigma_{x}^{2}\left(\begin{array}{llll}\frac{\beta_{1}}{\sigma_{1}^{2}} & \frac{\beta_{2}}{\sigma_{2}^{2}} & \cdots & \frac{\beta_{p}}{\sigma_{p}^{2}}\end{array}\right)\left(\begin{array}{c}\beta_{1} \\ \beta_{2} \\ \vdots \\ \beta_{p}\end{array}\right)+1\right)\right|$,

que pode ser escrito como:

$=\frac{1}{2}\left(\sum_{i=1}^{p} \ln \left(\sigma_{i}^{2}\right)\right)+\ln \left(\sum_{i=1}^{p} \frac{\sigma_{x}^{2} \beta_{i}^{2}}{\sigma_{i}^{2}}+1\right)$.

Note também que:

$$
\begin{aligned}
& I I=\Sigma^{-1}=\psi^{-1}-\frac{\sigma_{x}^{2} \psi^{-1} \beta \beta^{\prime} \psi^{-1}}{1+\beta^{\prime} \psi^{-1} \beta}, \\
& =\left(\begin{array}{cccc}
\frac{1}{\sigma_{1}^{2}} & 0 & \cdots & 0 \\
0 & \frac{1}{\sigma_{2}^{2}} & \cdots & 0 \\
\vdots & \vdots & \ddots & \vdots \\
0 & 0 & \cdots & \frac{1}{\sigma_{p}^{2}}
\end{array}\right)-\frac{\left(\begin{array}{cccc}
\frac{1}{\sigma_{1}^{2}} & 0 & \cdots & 0 \\
0 & \frac{1}{\sigma_{2}^{2}} & \cdots & 0 \\
\vdots & \vdots & \ddots & \vdots \\
0 & 0 & \cdots & \frac{1}{\sigma_{p}^{2}}
\end{array}\right)\left(\begin{array}{c}
\beta_{1} \\
\beta_{2} \\
\vdots \\
\beta_{p}
\end{array}\right)\left(\begin{array}{cccc}
\beta_{1} & \beta_{2} & \cdots & \beta_{p}
\end{array}\right)\left(\begin{array}{cccc}
\frac{1}{\sigma_{1}^{2}} & 0 & \cdots & 0 \\
0 & \frac{1}{\sigma_{2}^{2}} & \cdots & 0 \\
\vdots & & \\
\vdots & \ddots & \vdots \\
0 & \cdots & \frac{1}{\sigma_{p}^{2}}
\end{array}\right)}{1+\left(\begin{array}{cccc}
\beta_{1} & \beta_{2} & \cdots & \beta_{p}
\end{array}\right)\left(\begin{array}{cccc}
\frac{1}{\sigma_{1}^{2}} & 0 & \cdots & 0 \\
0 & \frac{1}{\sigma_{2}^{2}} & \cdots & 0 \\
\vdots & \vdots & \ddots & \vdots \\
0 & 0 & \cdots & \frac{1}{\sigma_{p}^{2}}
\end{array}\right)\left(\begin{array}{c}
\beta_{1} \\
\beta_{2} \\
\vdots \\
\beta_{p}
\end{array}\right)},
\end{aligned}
$$

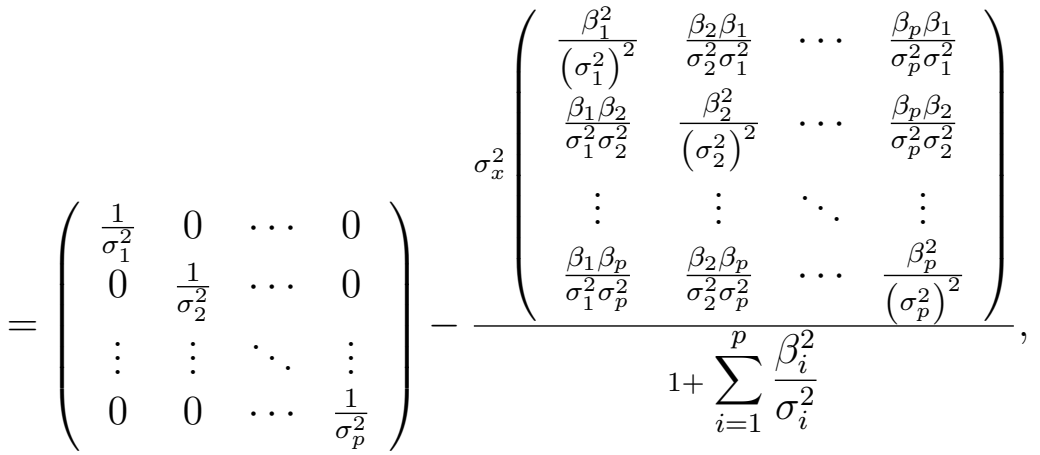


de modo que

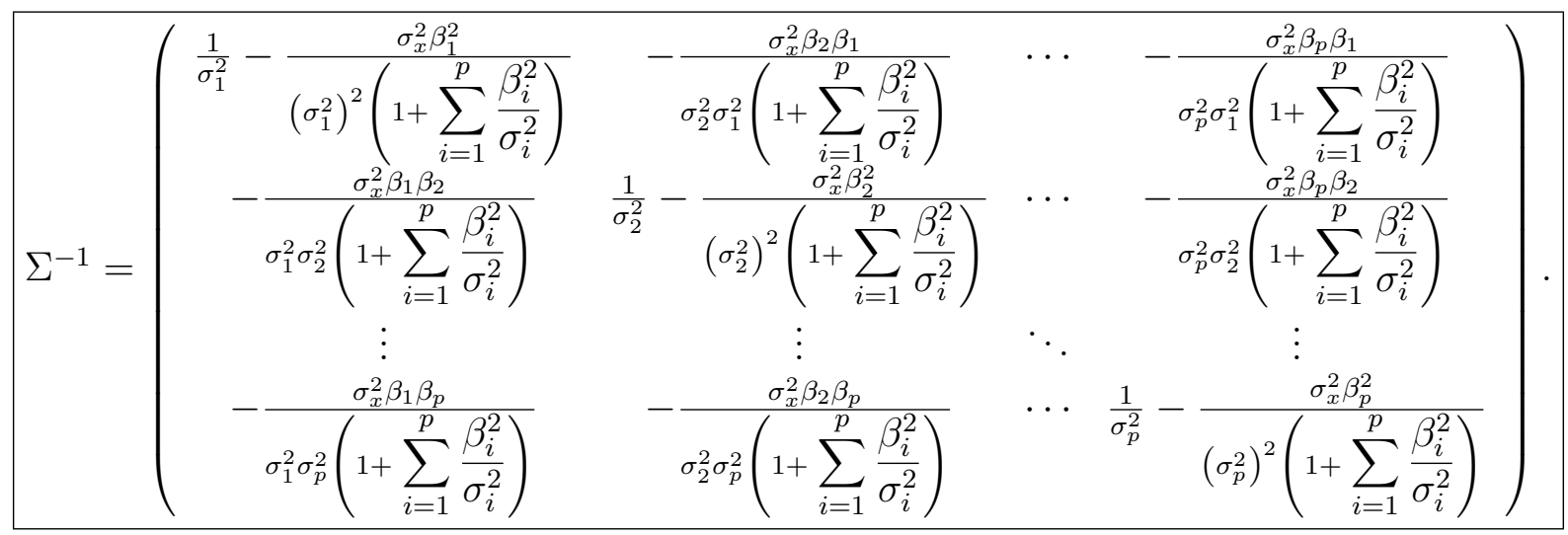

$$
\begin{aligned}
& I I I=\left(\mathbf{Y}_{j}-\mu\right)\left(\mathbf{Y}_{j}-\mu\right)^{\prime}, \\
& =\left(\begin{array}{c}
Y_{1 j}-\alpha_{1}-\beta_{1} \mu_{x} \\
Y_{2 j}-\alpha_{2}-\beta_{2} \mu_{x} \\
\vdots \\
Y_{p j}-\alpha_{p}-\beta_{p} \mu_{x}
\end{array}\right)\left(\begin{array}{llll}
Y_{1 j}-\alpha_{1}-\beta_{1} \mu_{x} & Y_{2 j}-\alpha_{2}-\beta_{2} \mu_{x} & \cdots & Y_{p j}-\alpha_{p}-\beta_{p} \mu_{x}
\end{array}\right), \\
& =\left(\begin{array}{cccc}
\left(Y_{1 j}-\alpha_{1}-\beta_{1} \mu_{x}\right)^{2} & \left(Y_{2 j}-\alpha_{2}-\beta_{2} \mu_{x}\right)\left(Y_{1 j}-\alpha_{1}-\beta_{1} \mu_{x}\right) & \cdots & \left(Y_{p j}-\alpha_{p}-\beta_{p} \mu_{x}\right)\left(Y_{1 j}-\alpha_{1}-\beta_{1} \mu_{x}\right. \\
\left(Y_{1 j}-\alpha_{1}-\beta_{1} \mu_{x}\right)\left(Y_{2 j}-\alpha_{2}-\beta_{2} \mu_{x}\right) & \left(Y_{2 j}-\alpha_{2}-\beta_{2} \mu_{x}\right)^{2} & \cdots & \left(Y_{p i}-\alpha_{p}-\beta_{p} \mu_{x}\right)\left(Y_{2 j}-\alpha_{2}-\beta_{2} \mu_{x}\right) \\
\vdots & \vdots & \ddots & \vdots \\
\left(Y_{1 j}-\alpha_{1}-\beta_{1} \mu_{x}\right)\left(Y_{p j}-\alpha_{p}-\beta_{p} \mu_{x}\right) & \left(Y_{2 j}-\alpha_{2}-\beta_{2} \mu_{x}\right)\left(Y_{p j}-\alpha_{p}-\beta_{p} \mu_{x}\right) & \cdots & \left(Y_{p j}-\alpha_{p}-\beta_{p} \mu_{x}\right)^{2}
\end{array}\right) \text {, }
\end{aligned}
$$

Por outro lado, multiplicando II por III temos:

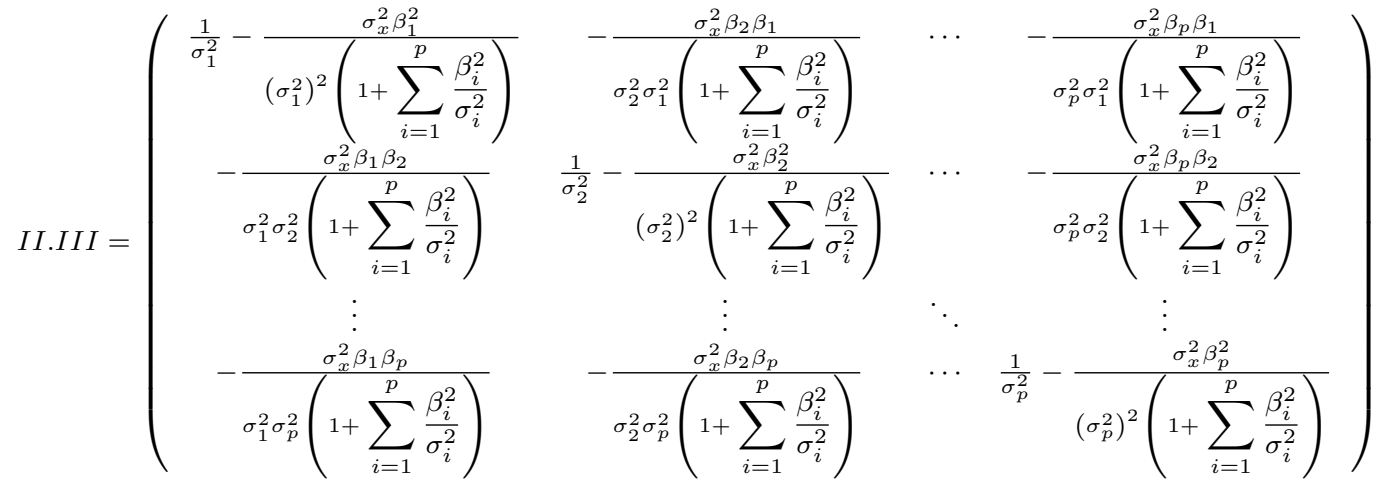

$$
\begin{aligned}
& \left(\begin{array}{c}
Y_{1 j}-\alpha_{1}-\beta_{1} \mu_{x} \\
Y_{2 j}-\alpha_{2}-\beta_{2} \mu_{x} \\
\vdots \\
Y_{p j}-\alpha_{p}-\beta_{p} \mu_{x}
\end{array}\right)\left(\begin{array}{llll}
Y_{1 j}-\alpha_{1}-\beta_{1} \mu_{x} & Y_{2 j}-\alpha_{2}-\beta_{2} \mu_{x} & \cdots & Y_{p j}-\alpha_{p}-\beta_{p} \mu_{x}
\end{array}\right) \text {, }
\end{aligned}
$$




$$
\begin{aligned}
& =\left(\begin{array}{c}
\frac{\left(Y_{1 j}-\alpha_{1}-\beta_{1} \mu_{x}\right)}{\sigma_{1}^{2}}-\sum_{i=1}^{p} \frac{\sigma_{x}^{2} \beta_{1} \beta_{i}\left(Y_{1 j}-\alpha_{1}-\beta_{1} \mu_{x}\right)}{\left(\sigma_{1}^{2}\right)^{2}\left(1+\sum_{i=1}^{p} \frac{\beta_{i}^{2}}{\sigma_{i}^{2}}\right)} \\
\frac{\left(Y_{2 j}-\alpha_{2}-\beta_{2} \mu_{x}\right)}{\sigma_{2}^{2}}-\sum_{i=1}^{p} \frac{\sigma_{x}^{2} \beta_{2} \beta_{i}\left(Y_{2 j}-\alpha_{2}-\beta_{2} \mu_{x}\right)}{\left(\sigma_{2}^{2}\right)^{2}\left(1+\sum_{i=1}^{p} \frac{\beta_{i}^{2}}{\sigma_{i}^{2}}\right)} \\
\vdots \\
\frac{\left(Y_{p j}-\alpha_{p}-\beta_{p} \mu_{x}\right)}{\sigma_{p}^{2}}-\sum_{i=1}^{p} \frac{\sigma_{x}^{2} \beta_{p} \beta_{i}\left(Y_{p j}-\alpha_{p}-\beta_{p} \mu_{x}\right)}{\left(\sigma_{p}^{2}\right)^{2}\left(1+\sum_{i=1}^{p} \frac{\beta_{i}^{2}}{\sigma_{i}^{2}}\right)}
\end{array}\right) \\
& \left(\begin{array}{ccc}
Y_{1 j}-\alpha_{1}-\beta_{1} \mu_{x} \quad Y_{2 j}-\alpha_{2}-\beta_{2} \mu_{x} \quad \cdots \quad Y_{p j}-\alpha_{p}-\beta_{p} \mu_{x}
\end{array}\right),
\end{aligned}
$$

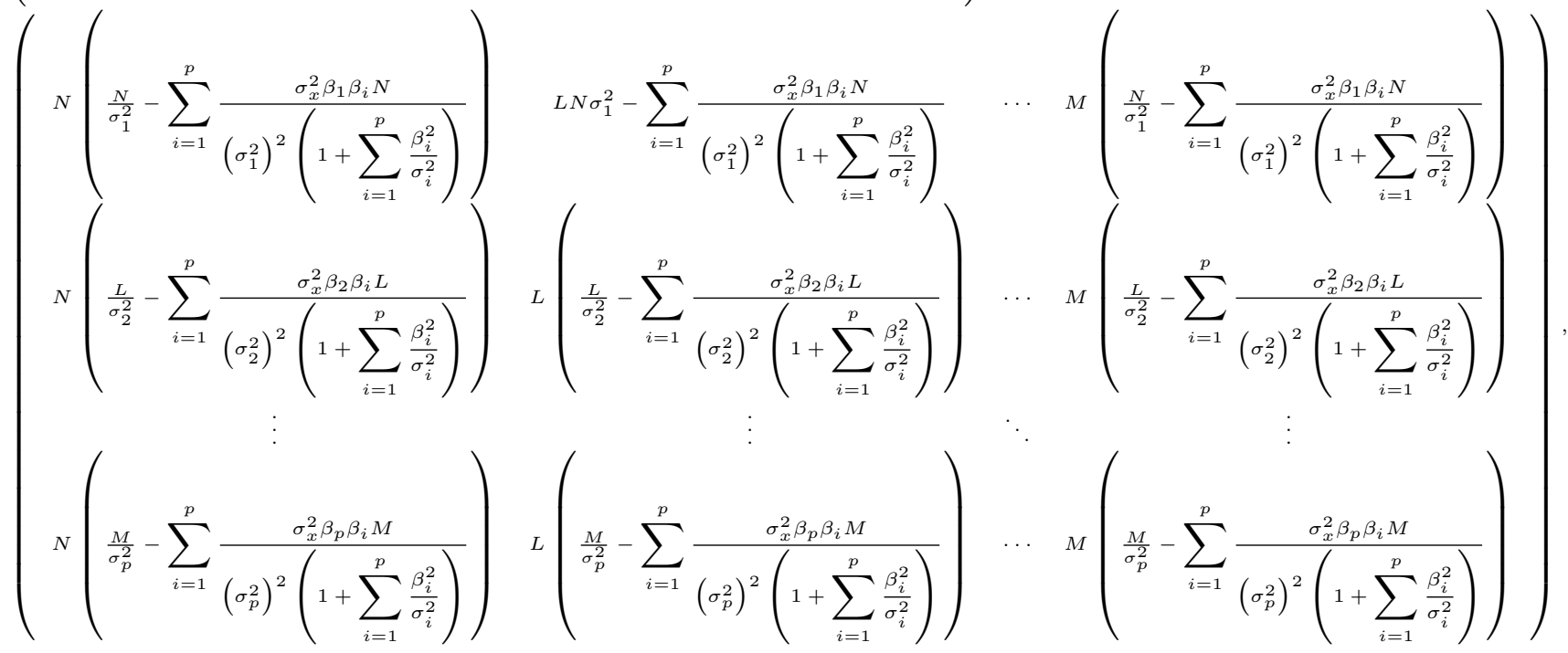

onde $N=\left(Y_{1 j}-\alpha_{1}-\beta_{1} \mu_{x}\right), L=\left(Y_{2 j}-\alpha_{2}-\beta_{2} \mu_{x}\right)$ e $M=\left(Y_{p j}-\alpha_{p}-\beta_{p} \mu_{x}\right)$.

Além disso, $\operatorname{tr}($ II.III) é dado por:

$$
\begin{aligned}
& \left(Y_{1 j}-\alpha_{1}-\beta_{1} \mu_{x}\right)\left(\frac{\left(Y_{1 j}-\alpha_{1}-\beta_{1} \mu_{x}\right)}{\sigma_{1}^{2}}-\sum_{i=1}^{p} \frac{\sigma_{x}^{2} \beta_{1} \beta_{i}\left(Y_{1 j}-\alpha_{1}-\beta_{1} \mu_{x}\right)}{\left(\sigma_{1}^{2}\right)^{2}\left(1+\sum_{i=1}^{p} \frac{\beta_{i}^{2}}{\sigma_{i}^{2}}\right)}\right)+ \\
& \left(Y_{2 j}-\alpha_{2}-\beta_{2} \mu_{x}\right)\left(\frac{\left(Y_{2 j}-\alpha_{2}-\beta_{2} \mu_{x}\right)}{\sigma_{2}^{2}}-\sum_{i=1}^{p} \frac{\sigma_{x}^{2} \beta_{2} \beta_{i}\left(Y_{2 j}-\alpha_{2}-\beta_{2} \mu_{x}\right)}{\left(\sigma_{2}^{2}\right)^{2}\left(1+\sum_{i=1}^{p} \frac{\beta_{i}^{2}}{\sigma_{i}^{2}}\right)}\right)+\cdots+ \\
& \left(Y_{p j}-\alpha_{p}-\beta_{p} \mu_{x}\right)\left(\frac{\left(Y_{p j}-\alpha_{p}-\beta_{p} \mu_{x}\right)}{\sigma_{p}^{2}}-\sum_{i=1}^{p} \frac{\sigma_{x}^{2} \beta_{p} \beta_{i}\left(Y_{p j}-\alpha_{p}-\beta_{p} \mu_{x}\right)}{\left(\sigma_{p}^{2}\right)^{2}\left(1+\sum_{i=1}^{p} \frac{\beta_{i}^{2}}{\sigma_{i}^{2}}\right)}\right) \text {, }
\end{aligned}
$$




$$
\begin{gathered}
=\frac{\left(Y_{1 j}-\alpha_{1}-\beta_{1} \mu_{x}\right)^{2}}{\sigma_{1}^{2}}-\frac{\sigma_{x}^{2} \beta_{1}\left(Y_{1 j}-\alpha_{1}-\beta_{1} \mu_{x}\right)^{2}}{\left(\sigma_{1}^{2}\right)^{2}} \sum_{i=1}^{p} \frac{\beta_{i}}{\left(1+\sum_{i=1}^{p} \frac{\beta_{i}^{2}}{\sigma_{i}^{2}}\right)}+ \\
\frac{\left(Y_{2 j}-\alpha_{2}-\beta_{2} \mu_{x}\right)^{2}}{\sigma_{2}^{2}}-\frac{\sigma_{x}^{2} \beta_{2}\left(Y_{2 j}-\alpha_{2}-\beta_{2} \mu_{x}\right)^{2}}{\left(\sigma_{2}^{2}\right)^{2}} \sum_{i=1}^{p} \frac{\beta_{i}}{\left(1+\sum_{i=1}^{p} \frac{\beta_{i}^{2}}{\sigma_{i}^{2}}\right)}+\cdots+ \\
\frac{\left(Y_{p j}-\alpha_{p}-\beta_{p} \mu_{x}\right)^{2}}{\sigma_{p}^{2}}-\frac{\sigma_{x}^{2} \beta_{p}\left(Y_{p j}-\alpha_{p}-\beta_{p} \mu_{x}\right)^{2}}{\left(\sigma_{p}^{2}\right)^{2}} \sum_{i=1}^{p} \frac{\beta_{i}}{\left(1+\sum_{i=1}^{p} \frac{\beta_{i}^{2}}{\sigma_{i}^{2}}\right)}, \\
\sum_{i=1}^{p}\left[\frac{\left(Y_{i j}-\alpha_{i}-\beta_{i} \mu_{x}\right)^{2}}{\sigma_{i}^{2}}\right]-\sum_{i=1}^{p}\left[\frac{\sigma_{x}^{2} \beta_{i}\left(Y_{i j}-\alpha_{i}-\beta_{i} \mu_{x}\right)^{2}}{\left(\sigma_{i}^{2}\right)^{2}}\right] \sum_{i=1}^{p}\left[\frac{\beta_{i}}{\left(1+\sum_{i=1}^{p} \frac{\beta_{i}^{2}}{\sigma_{i}^{2}}\right)}\right],
\end{gathered}
$$

Agora, para ln $L(\theta)$ temos:

$$
\begin{gathered}
\ln (\theta)=-n\left[\ln (2 \pi)+\frac{1}{2}\left(\sum_{i=1}^{p} \ln \left(\sigma_{i}^{2}\right)\right)+\frac{1}{2} \ln \left(\sum_{i=1}^{p} \frac{\sigma_{x}^{2} \beta_{i}^{2}}{\sigma_{i}^{2}}+1\right)\right] \\
-\frac{1}{2} \sum_{j=1}^{n}\left[\sum_{i=1}^{p}\left[\frac{\left(Y_{i j}-\alpha_{i}-\beta_{i} \mu_{x}\right)^{2}}{\sigma_{i}^{2}}\right]-\sum_{i=1}^{p}\left[\frac{\sigma_{x}^{2} \beta_{i}\left(Y_{i j}-\alpha_{i}-\beta_{i} \mu_{x}\right)^{2}}{\left(\sigma_{i}^{2}\right)^{2}}\right] \sum_{i=1}^{p}\left[\frac{\beta_{i}}{\left.\left(1+\sum_{i=1}^{p} \frac{\beta_{i}^{2}}{\sigma_{i}^{2}}\right)\right]},\right.\right.
\end{gathered}
$$

que fica:

$$
\begin{gathered}
\ln (L(\theta))=-n\left[\ln (2 \pi)+\frac{1}{2}\left(\sum_{i=1}^{p} \ln \left(\sigma_{i}^{2}\right)\right)+\frac{1}{2} \ln \left(\sum_{i=1}^{p} \frac{\sigma_{x}^{2} \beta_{i}^{2}}{\sigma_{i}^{2}}+1\right)\right] \\
-\left(\frac{1}{2}\right) \sum_{j=1}^{n}\left[\sum_{i=1}^{p}\left[\frac{\left(Y_{i j}-\alpha_{i}-\beta_{i} \mu_{x}\right)^{2}}{\sigma_{i}^{2}}\right]-\sum_{i=1}^{p}\left[\frac{\sigma_{x}^{2} \beta_{i}\left(Y_{i j}-\alpha_{i}-\beta_{i} \mu_{x}\right)^{2}}{\left(\sigma_{i}^{2}\right)^{2}}\right] \frac{\sum_{i=1}^{p} \beta_{i}}{\left.\left(1+\sum_{i=1}^{p} \frac{\beta_{i}^{2}}{\sigma_{i}^{2}}\right)\right] .}\right.
\end{gathered}
$$




\section{Apêndice C}

\section{Teste da razão de verossimilhanças}

Consideremos a seguir a obtenção do teste da razão de verossimilhança para verificar os seguintes casos:

- caso 1 - verificar se os $p$ instrumentos medem a característica sem vício.

- caso 2 - verificar se os viéses são iguais para os mesmos $p$ instrumentos.

Para que seja possível fazer esse teste, foi considerada uma população pvariada, considerando as condições e suposições estabelecidas no capítulo 2.1 e apêndice B.

Para efeito de notação, foi considerado 01 para a função de verossimilhança da primeira hipótese de interesse e 02 para a função de verossimilhança para a segunda hipótese de interesse.

\section{C.1 Função de verossimilhança para os dados obser- vados.}

\section{C.1.1 Modelo sem restrição}

Para os dados observados, temos a seguinte função de verossimilhança.

$$
L(\theta)=\frac{1}{(2 \pi)^{n}(|\Sigma|)^{\frac{n}{2}}} \exp \left[-\frac{1}{2} \sum_{j=1}^{n}\left(Y_{j}-\mu_{Y}\right)^{\prime} \Sigma^{-1}\left(Y_{j}-\mu_{Y}\right)\right]
$$

utilizando o resultado A.1 do apêndice A podemos expressar como:

$$
L(\theta)=\frac{1}{(2 \pi)^{n}(|\Sigma|)^{\frac{n}{2}}} \exp \left\{-\frac{1}{2} \sum_{j=1}^{n} \operatorname{tr}\left[\Sigma^{-1}\left(Y_{j}-\mu_{Y}\right)\left(Y_{j}-\mu_{Y}\right)^{\prime}\right]\right\}
$$




$$
\begin{gathered}
L(\theta)=\frac{1}{(2 \pi)^{n}(|\Sigma|)^{\frac{n}{2}}} \exp \left\{-\frac{1}{2} \operatorname{tr}\left[\Sigma^{-1} \sum_{j=1}^{n}\left(Y_{j}-\mu_{Y}\right)\left(Y_{j}-\mu_{Y}\right)^{\prime}\right]\right\}, \\
L(\theta)=\frac{1}{(2 \pi)^{n}(|\Sigma|)^{\frac{n}{2}}} \exp \left\{-\frac{1}{2} t r\left[\Sigma^{-1} \sum_{j=1}^{n}\left[\left(\begin{array}{c}
Y_{1 j}-\alpha_{1}-\beta_{1} \mu_{x} \\
\vdots \\
Y_{p j}-\alpha_{p}-\beta_{p} \mu_{x}
\end{array}\right)\left(\begin{array}{lll}
Y_{1 j}-\alpha_{1}-\beta_{1} \mu_{x} & \cdots & Y_{p j}-\alpha_{p}-\beta_{p} \mu_{x}
\end{array}\right)^{\prime}\right]\right]\right\},
\end{gathered}
$$

onde $\Sigma e \boldsymbol{\Sigma}^{-\mathbf{1}}$ são dada no apêndice B.

\section{C.1.2 Função de verossimilhança para a primeira hipótese de interesse}

Para a hipótese; $H_{01}: \alpha_{2}=\cdots=\alpha_{p}=0 ; \beta_{2}=\cdots=\beta_{p}=1$ temos a seguinte função de verossimilhança:

$L\left(\theta_{01}\right)=\frac{1}{(2 \pi)^{n}\left(\left|\Sigma_{01}\right|\right)^{\frac{n}{2}}} \exp \left\{-\frac{1}{2} \operatorname{tr}\left[\Sigma_{01}^{-1} \sum_{j=1}^{n}\left[\left(\begin{array}{c}Y_{1 j}-\mu_{x_{01}} \\ \vdots \\ Y_{p j}-\mu_{x_{01}}\end{array}\right)\left(\begin{array}{lll}Y_{1 j}-\mu_{x_{01}} & \cdots & Y_{p j}-\mu_{x_{01}}\end{array}\right)^{\prime}\right]\right]\right\}$

com:

$$
\Sigma_{01}=\left(\begin{array}{ccccc}
\sigma_{x}^{2}+\sigma_{1}^{2} & \sigma_{x}^{2} & \sigma_{x}^{2} & \cdots & \sigma_{x}^{2} \\
\sigma_{x}^{2} & \sigma_{x}^{2}+\sigma_{2}^{2} & \sigma_{x}^{2} & \cdots & \sigma_{x}^{2} \\
\sigma_{x}^{2} & \sigma_{x}^{2} & \sigma_{x}^{2}+\sigma_{3}^{2} & \cdots & \sigma_{x}^{2} \\
\vdots & \vdots & \vdots & \ddots & \vdots \\
\sigma_{x}^{2} & \sigma_{x}^{2} & \sigma_{x}^{2} & \cdots & \sigma_{x}^{2}+\sigma_{p}^{2}
\end{array}\right)
$$

e

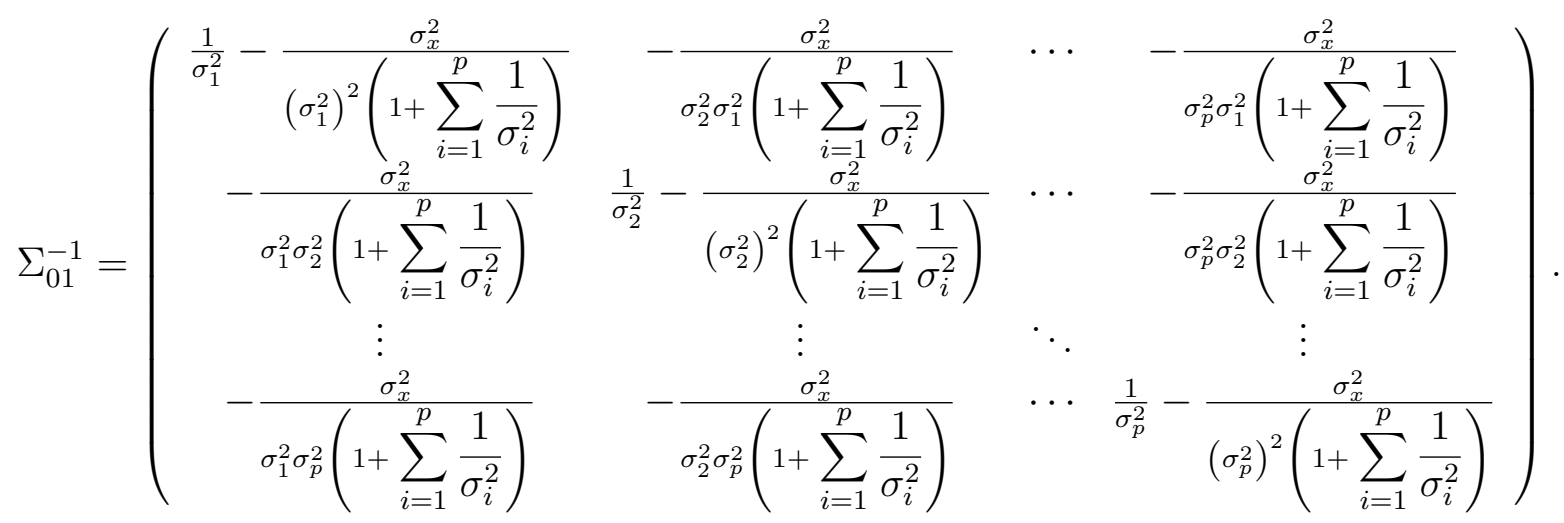




\section{C.1.3 Razão de verossimilhanças para a primeira hipótese de interesse}

Como visto anteriormente, a estatística da razão de verossimilhanças é dada por:

$$
\lambda_{1}=\frac{L\left(\hat{\theta}_{01} ; X\right)}{L(\hat{\theta} ; X)},
$$

que resulta em:

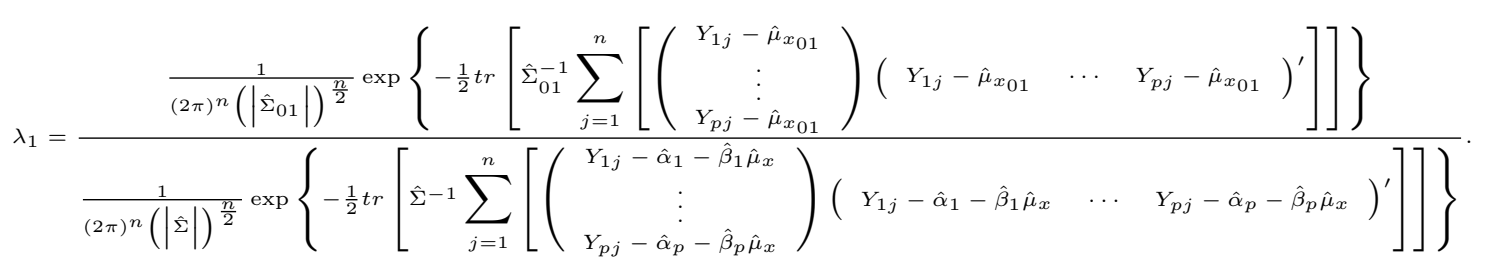

\section{C.1.4 Função de verossimilhança para a segunda hipótese de interesse}

Para a hipótese; $H_{02}: \alpha_{2}=\cdots=\alpha_{p}=\alpha ; \beta_{2}=\cdots=\beta_{p}=\beta$ temos a seguinte função de verossimilhança:

$L\left(\theta_{02}\right)=\frac{1}{(2 \pi)^{n}\left(\left|\Sigma_{02}\right|\right)^{\frac{n}{2}} \exp }\left\{-\frac{1}{2} \operatorname{tr}\left[\Sigma_{02}^{-1} \sum_{j=1}^{n}\left[\left(\begin{array}{c}Y_{1 j}-\alpha_{02}-\beta_{02} \mu_{02} \\ \vdots \\ Y_{p j}-\alpha_{02}-\beta_{02} \mu_{x_{02}}\end{array}\right)\left(\begin{array}{lll}Y_{1 j}-\alpha_{02}-\beta_{02} \mu_{02} & \cdots & Y_{p j}-\alpha_{02}-\beta_{02} \mu_{02}\end{array}\right)^{\prime}\right]\right]\right\}$, com:

$$
\Sigma_{02}=\left(\begin{array}{ccccc}
\beta^{2} \sigma_{x}^{2}+\sigma_{1}^{2} & \beta^{2} \sigma_{x}^{2} & \beta^{2} \sigma_{x}^{2} & \cdots & \beta^{2} \sigma_{x}^{2} \\
\beta^{2} \sigma_{x}^{2} & \beta^{2} \sigma_{x}^{2}+\sigma_{2}^{2} & \beta^{2} \sigma_{x}^{2} & \cdots & \beta^{2} \sigma_{x}^{2} \\
\beta^{2} \sigma_{x}^{2} & \beta^{2} \sigma_{x}^{2} & \beta^{2} \sigma_{x}^{2}+\sigma_{3}^{2} & \cdots & \beta^{2} \sigma_{x}^{2} \\
\vdots & \vdots & \vdots & \ddots & \vdots \\
\beta^{2} \sigma_{x}^{2} & \beta^{2} \sigma_{x}^{2} & \beta^{2} \sigma_{x}^{2} & \cdots & \beta^{2} \sigma_{x}^{2}+\sigma_{p}^{2}
\end{array}\right)
$$

e 


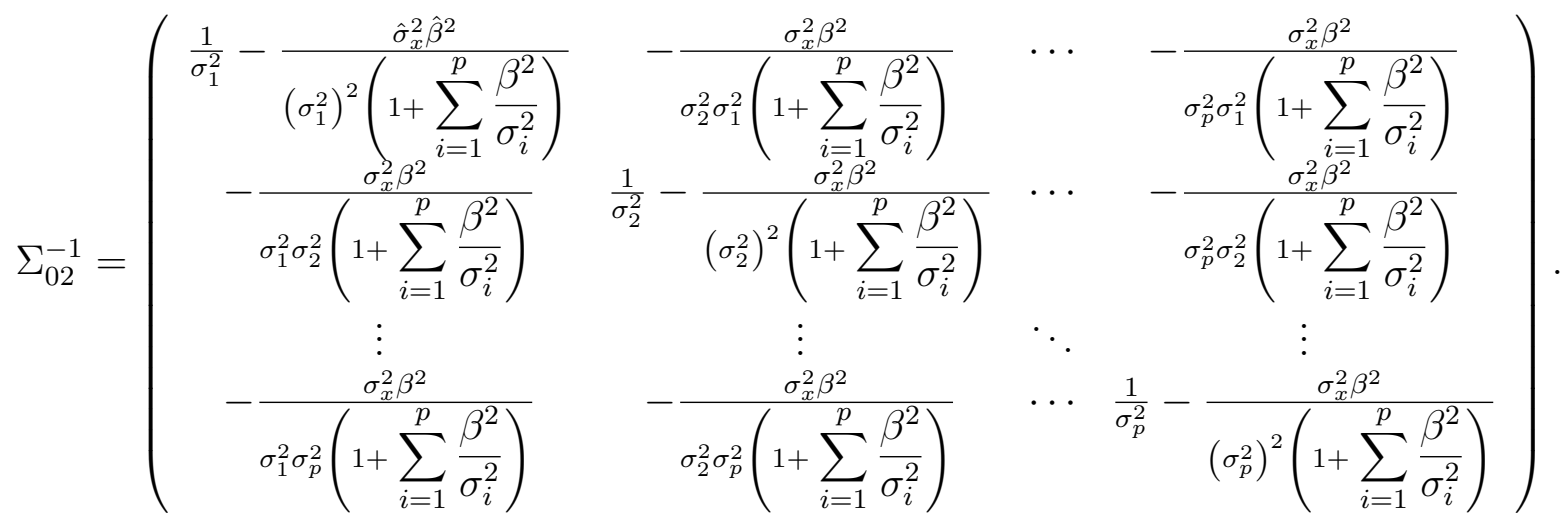

\section{C.1.5 Razão de verossimilhanças para a segunda hipótese de interesse}

Neste caso a estatística é dada por:

$$
\lambda_{2}=\frac{L\left(\hat{\theta}_{02} ; X\right)}{L(\hat{\theta} ; X)},
$$

que resulta em:

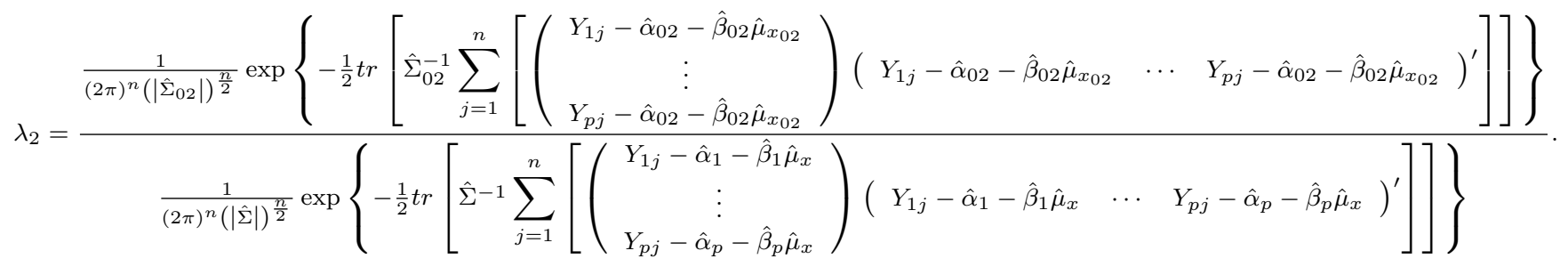

O cálculo dos estimadores $\hat{\theta}_{02}$ e $\hat{\theta}$ obtidos pela verossimilhança maximizada deve ser feito utilizando procedimentos computacionais. 


\section{Apêndice D}

\section{Função de verossimilhança completa}

Agora, para os dados completos (incorpora as variáveis não observadas), a função de verossimilhança pode ser escrita a partir de uma distribuição normal $p$ variada com função densidade dada por:

$$
f(\theta / Z)=\frac{1}{2 \pi\left|\Sigma_{Z}\right|^{\frac{1}{2}}} \exp \left[-\frac{1}{2}\left(Z-\mu_{Z}\right)^{\prime} \Sigma_{Z}^{-1}\left(Z-\mu_{Z}\right)\right]
$$

com vetor de médias e matriz de covariâncias dadas, respectivamente, por:

$$
\mu_{Z}=\left(\begin{array}{c}
\mu_{x} \\
\alpha_{1}+\beta_{1} \mu_{x} \\
\vdots \\
\alpha_{p}+\beta_{p} \mu_{x}
\end{array}\right) \text { e } \Sigma_{Z}=\sigma_{x}^{2}\left(\begin{array}{cc}
1 & \beta^{\prime} \\
\beta & \beta \beta^{\prime}+A
\end{array}\right)
$$

$\operatorname{com} A=\operatorname{diag}\left(\frac{\sigma_{1}^{2}}{\sigma_{x}^{2}}, \cdots, \frac{\sigma_{p}^{2}}{\sigma_{x}^{2}}\right)$.

Assim, para o caso dos dados completos obtemos o seguinte logarítmo da função de verossimilhança:

$$
\begin{gathered}
\ln (L(\theta / Z))=-n \ln \left(2 \pi\left|\Sigma_{Z}\right|^{\frac{1}{2}}\right)-\frac{1}{2} \sum_{j=1}^{n} \operatorname{tr}\left[\Sigma_{Z}^{-1}\left(Z_{j}-\mu_{Z}\right)\left(Z_{j}-\mu_{Z}\right)^{\prime}\right] \\
=-n \ln (2 \pi)-\frac{n}{2} \ln \left(\left|\Sigma_{Z}\right|\right)-\frac{1}{2} \sum_{j=1}^{n} \operatorname{tr}\left[\Sigma_{Z}^{-1}\left(Z_{j}-\mu_{Z}\right)\left(Z_{j}-\mu_{Z}\right)^{\prime}\right] .
\end{gathered}
$$


Seja $I=\left|\Sigma_{Z}\right|, I I=\Sigma_{Z}^{-1}$ e $I I I=\left(Z_{j}-\mu_{Z}\right)\left(Z_{j}-\mu_{Z}\right)^{\prime}$, de modo que:

$$
\begin{aligned}
& I=\left|\Sigma_{Z}\right|=\left|\sigma_{x}^{2}\right| \cdot\left|\begin{array}{cc}
1 & \beta^{\prime} \\
\beta & \beta \beta^{\prime}+A
\end{array}\right|=\sigma_{x}^{2}\left|\beta \beta^{\prime}+A-\beta \beta^{\prime}\right|=\sigma_{x}^{2}|A|=\sigma_{x}^{2} \prod_{i=1}^{p} \sigma_{i}^{2}, \\
& I I=\Sigma_{Z}^{-1}=\left(\sigma_{x}^{2} \cdot\left(\begin{array}{cc}
1 & \beta^{\prime} \\
\beta & \beta \beta^{\prime}+A
\end{array}\right)\right)^{-1}=\frac{1}{\sigma_{x}^{2}}\left(\begin{array}{cc}
1 & \beta^{\prime} \\
\beta & \beta \beta^{\prime}+A
\end{array}\right)^{-1} .
\end{aligned}
$$

Seja $A_{11}=1, A_{12}=\beta^{\prime}, A_{21}=\beta$ e $A_{22}=\beta \beta^{\prime}+A$ :

$$
\begin{aligned}
& A^{11}=\left(\sigma_{x}^{2}\right)^{-1}\left[1-\beta^{\prime}\left(\beta \beta^{\prime}+A\right)^{-1} \beta\right]^{-1}, \\
& =\left(\sigma_{x}^{2}\right)^{-1}\left[1-\beta^{\prime}\left(A^{-1}-\frac{A^{-1} \beta \beta^{\prime} A^{-1}}{1+\beta^{\prime} A^{-1} \beta}\right) \beta\right]^{-1}, \\
& =\left(\sigma_{x}^{2}\right)^{-1}\left[\begin{array}{cccc}
\sigma_{x}^{2} & 0 & \cdots & 0 \\
\sigma_{1}^{2} & & \\
0 & \frac{\sigma_{x}^{2}}{\sigma_{2}^{2}} & \cdots & 0 \\
\vdots & \vdots & \ddots & \vdots \\
0 & 0 & \cdots & \frac{\sigma_{x}^{2}}{\sigma_{p}^{2}}
\end{array}\right)-\left(\begin{array}{cccc}
\frac{\sigma_{x}^{2}}{\sigma_{1}^{2}} & 0 & \cdots & 0 \\
0 & \frac{\sigma_{x}^{2}}{\sigma_{2}^{2}} & \cdots & 0 \\
\vdots & \vdots & \ddots & \vdots \\
0 & 0 & \cdots & \frac{\sigma_{x}^{2}}{\sigma_{p}^{2}}
\end{array}\right)\left(\begin{array}{ccccc}
\frac{\sigma_{x}^{2}}{\sigma_{1}^{2}} & 0 & \cdots & 0 \\
0 & \frac{\sigma_{x}^{2}}{\sigma_{2}^{2}} & \cdots & 0 \\
\vdots & \vdots & \ddots & \vdots \\
0 & 0 & \cdots & \frac{\sigma_{x}^{2}}{\sigma_{p}^{2}}
\end{array}\right) \\
& \\
&
\end{aligned}
$$

que resolvendo obtemos o seguinte resultado:

$$
\begin{aligned}
& =\left(\sigma_{x}^{2}\right)^{-1}\left[1-\beta^{\prime}\left(\begin{array}{cccc}
\frac{\sigma_{x}^{2}}{\sigma_{1}^{2}}-\frac{\left(\sigma_{x}^{2} \beta_{1}\right)^{2}}{K\left(\sigma_{1}^{2}\right)^{2}} & -\frac{\sigma_{x}^{2} \beta_{2}}{\sigma_{2}^{2}} \frac{\sigma_{x}^{2} \beta_{1}}{K \sigma_{1}^{2}} & \cdots & -\frac{\sigma_{x}^{2} \beta_{p}}{\sigma_{p}^{2}} \frac{\sigma_{x}^{2} \beta_{1}}{K \sigma_{1}^{2}} \\
-\frac{\sigma_{x}^{2} \beta_{1}}{\sigma_{1}^{2}} \frac{\sigma_{x}^{2} \beta_{2}}{K \sigma_{2}^{2}} & \frac{\sigma_{x}^{2}}{\sigma_{2}^{2}}-\frac{\left(\sigma_{x}^{2} \beta_{2}\right)^{2}}{K\left(\sigma_{2}^{2}\right)^{2}} & \cdots & -\frac{\sigma_{x}^{2} \beta_{p}}{\sigma_{p}^{2}} \frac{\sigma_{x}^{2} \beta_{2}}{K \sigma_{2}^{2}} \\
\vdots & \vdots & \ddots & \vdots \\
-\frac{\sigma_{x}^{2} \beta_{1}}{\sigma_{1}^{2}} \frac{\sigma_{x}^{2} \beta_{p}}{K \sigma_{p}^{2}} & -\frac{\sigma_{x}^{2} \beta_{2}}{\sigma_{2}^{2}} \frac{\sigma_{x}^{2} \beta_{p}}{K \sigma_{p}^{2}} & \cdots & \frac{\sigma_{x}^{2}}{\sigma_{p}^{2}}-\frac{\left(\sigma_{x}^{2} \beta_{p}\right)^{2}}{K\left(\sigma_{p}^{2}\right)^{2}}
\end{array}\right]^{-1}\right]^{-} \\
& =\left(\sigma_{x}^{2}\right)^{-1}\left[1-\left(=\frac{\sigma_{x}^{2} \beta_{1}}{\sigma_{1}^{2}}-\frac{\beta_{1}}{K \sigma_{1}^{2}} \sum_{i=1}^{p} \frac{\left(\sigma_{x}^{2} \beta_{i}\right)^{2}}{\sigma_{i}^{2}} \quad \frac{\sigma_{x}^{2} \beta_{2}}{\sigma_{2}^{2}}-\frac{\beta_{2}}{K \sigma_{2}^{2}} \sum_{i=1}^{p} \frac{\left(\sigma_{x}^{2} \beta_{i}\right)^{2}}{\sigma_{i}^{2}} \quad \ldots \quad \frac{\sigma_{x}^{2} \beta_{p}}{\sigma_{p}^{2}}-\frac{\beta_{p}}{K \sigma_{p}^{2}} \sum_{i=1}^{p} \frac{\left(\sigma_{x}^{2} \beta_{i}\right)^{2}}{\sigma_{i}^{2}}\right)^{\beta}\right]^{-1}, \\
& =\left(\sigma_{x}^{2}\right)^{-1}\left[1-\left(\sum_{i=1}^{p} \frac{\sigma_{x}^{2}\left(\beta_{i}\right)^{2}}{\sigma_{i}^{2}}-\left(\frac{\left(\beta_{1}\right)^{2}}{K \sigma_{1}^{2}}+\frac{\left(\beta_{2}\right)^{2}}{K \sigma_{2}^{2}}+\cdots+\frac{\left(\beta_{p}\right)^{2}}{K \sigma_{p}^{2}}\right) \sum_{i=1}^{p} \frac{\left(\sigma_{x}^{2} \beta_{i}\right)^{2}}{\sigma_{i}^{2}}\right)\right]^{-1} .
\end{aligned}
$$

Então, 


$$
\begin{aligned}
& A^{11}=\left(\sigma_{x}^{2}\right)^{-1}\left[1-\left(\sum_{i=1}^{p} \frac{\sigma_{x}^{2}\left(\beta_{i}\right)^{2}}{\sigma_{i}^{2}}-\sum_{i=1}^{p} \frac{\left(\beta_{i}\right)^{2}}{K \sigma_{i}^{2}} \sum_{i=1}^{p} \frac{\left(\sigma_{x}^{2} \beta_{i}\right)^{2}}{\sigma_{i}^{2}}\right)\right]^{-1}, \\
& =\left(\sigma_{x}^{2}\right)^{-1}\left[1-\left(K-1-\frac{(K-1)}{K}(K-1)\right]^{-1}=\left(\sigma_{x}^{2}\right)^{-1}\left[1-\left(K-1-\frac{(K-1)^{2}}{K}\right)\right]^{-1},\right.
\end{aligned}
$$

que resolvendo, chegamos em:

$$
\begin{aligned}
& A^{11}=\left(\sigma_{x}^{2}\right)^{-1}\left[\frac{1}{K}\right]^{-1}=\left(\sigma_{x}^{2}\right)^{-1} K=\frac{K}{\sigma_{x}^{2}} \text {. } \\
& A^{22}=\left(\sigma_{x}^{2}\right)^{-1}\left(A_{22}-A_{21} A_{11}^{-1} A_{12}\right)^{-1} \text {, } \\
& =\left(\sigma_{x}^{2}\right)^{-1}\left(\beta \beta^{\prime}+A-\beta .1 . \beta^{\prime}\right)^{-1}=\left(\sigma_{x}^{2}\right)^{-1}(A)^{-1}= \\
& \left(\sigma_{x}^{2}\right)^{-1}\left(\begin{array}{cccc}
\frac{\sigma_{x}^{2}}{\sigma_{1}^{2}} & 0 & \cdots & 0 \\
0 & \frac{\sigma_{x}^{2}}{\sigma_{2}^{2}} & \cdots & 0 \\
\vdots & \vdots & \ddots & \vdots \\
0 & 0 & \cdots & \frac{\sigma_{x}^{2}}{\sigma_{p}^{2}}
\end{array}\right) \\
& A^{22}=\left(\begin{array}{cccc}
\frac{1}{\sigma_{1}^{2}} & 0 & \cdots & 0 \\
0 & \frac{1}{\sigma_{2}^{2}} & \cdots & 0 \\
\vdots & \vdots & \ddots & \vdots \\
0 & 0 & \cdots & \frac{1}{\sigma_{p}^{2}}
\end{array}\right) \text {. } \\
& A^{21}=-\left(\sigma_{x}^{2}\right)^{-1} A^{22} A_{21} A_{11}^{-1}=-\left(\sigma_{x}^{2}\right)^{-1}\left(\begin{array}{cccc}
\frac{\sigma_{x}^{2}}{\sigma_{1}^{2}} & 0 & \cdots & 0 \\
0 & \frac{\sigma_{x}^{2}}{\sigma_{2}^{2}} & \cdots & 0 \\
\vdots & \vdots & \ddots & \vdots \\
0 & 0 & \cdots & \frac{\sigma_{x}^{2}}{\sigma_{p}^{2}}
\end{array}\right) \beta .1=-\left(\begin{array}{c}
\frac{\beta_{1}}{\sigma_{1}^{2}} \\
\frac{\beta_{2}}{\sigma_{2}^{2}} \\
\vdots \\
\frac{\beta_{p}}{\sigma_{p}^{2}}
\end{array}\right) .
\end{aligned}
$$$$
A^{12}=-\left(\sigma_{x}^{2}\right)^{-1} A_{11}^{-1} A_{12} A^{22}=-\left(\sigma_{x}^{2}\right)^{-1} 1 \beta^{\prime}\left(\begin{array}{cccc}
\frac{\sigma_{x}^{2}}{\sigma_{1}^{2}} & 0 & \cdots & 0 \\
0 & \frac{\sigma_{x}^{2}}{\sigma_{2}^{2}} & \cdots & 0 \\
\vdots & \vdots & \ddots & \vdots \\
0 & 0 & \cdots & \frac{\sigma_{x}^{2}}{\sigma_{p}^{2}}
\end{array}\right)=-\left(\begin{array}{cccc}
\frac{\beta_{1}}{\sigma_{1}^{2}} & \frac{\beta_{2}}{\sigma_{2}^{2}} & \cdots & \frac{\beta_{p}}{\sigma_{p}^{2}}
\end{array}\right) \text {. }
$$

onde $K=1+\sigma_{x}^{2} \frac{\beta_{i}^{2}}{\sigma_{i}^{2}}$ e é importante salientar que esse mesmo valor de $K$ é utilizado em todo o apêndice $\mathrm{D}$ e também em todo o apêndice E.

Então,

$$
\begin{gathered}
\Sigma_{z}^{-1}=\left(\begin{array}{ccccc}
\frac{K}{\sigma_{x}^{2}} & -\frac{\beta_{1}}{\sigma_{1}^{2}} & -\frac{\beta_{2}}{\sigma_{2}^{2}} & \cdots & -\frac{\beta_{p}}{\sigma_{p}^{2}} \\
-\frac{\beta_{1}}{\sigma_{1}^{2}} & \frac{1}{\sigma_{1}^{2}} & 0 & \cdots & 0 \\
-\frac{\beta_{2}}{\sigma_{2}^{2}} & 0 & \frac{1}{\sigma_{2}^{2}} & \cdots & 0 \\
\vdots & \vdots & \vdots & \ddots & \vdots \\
-\frac{\beta_{p}}{\sigma_{p}^{2}} & 0 & 0 & \cdots & \frac{1}{\sigma_{p}^{2}}
\end{array}\right) . \\
I I I=\left(Z_{j}-\mu_{Z}\right)\left(Z_{j}-\mu_{Z}\right)^{\prime},
\end{gathered}
$$




$$
\begin{aligned}
& =\left(\begin{array}{c}
x_{j}-\mu_{x} \\
Y_{1 j}-\alpha_{1}-\beta_{1} \mu_{x} \\
Y_{2 j}-\alpha_{2}-\beta_{2} \mu_{x} \\
\vdots \\
Y_{p j}-\alpha_{p}-\beta_{p} \mu_{x}
\end{array}\right)\left(\begin{array}{ccccc}
x_{j}-\mu_{x} & Y_{1 j}-\alpha_{1}-\beta_{1} \mu_{x} & Y_{2 j}-\alpha_{2}-\beta_{2} \mu_{x} & \cdots & Y_{p j}-\alpha_{p}-\beta_{p} \mu_{x}
\end{array}\right)^{\prime}, \\
& =\left(\begin{array}{ccccc}
A^{2} & B A & C A & \cdots & D A \\
B A & B^{2} & C B & \cdots & D B \\
C A & C B & C^{2} & \cdots & D C \\
\vdots & \vdots & \vdots & \ddots & \vdots \\
D A & D B & C D & \cdots & D^{2}
\end{array}\right) .
\end{aligned}
$$

Por outro lado, para obter o traço do produto de II por III, primeiramente calculamos os valores das caselas $c_{11}, c_{22}, \cdots, c_{p p}$ do produto de II por III que obtemos:

$$
\begin{aligned}
& c_{11}=\left(\frac{K}{\sigma_{x}^{2}} A^{2}-\frac{\beta_{1} B A}{\sigma_{1}^{2}}-\frac{\beta_{2} C A}{\sigma_{2}^{2}} \cdots-\frac{\beta_{p} D A}{\sigma_{p}^{2}}\right) \\
& c_{11}=\frac{K A^{2}}{\left(\sigma_{x}^{2}\right)}-\frac{\beta_{1} B A}{\sigma_{1}^{2}}-\frac{\beta_{2} C A}{\sigma_{2}^{2}} \cdots-\frac{\beta_{p} D A}{\sigma_{p}^{2}}, \\
& c_{11}=\frac{K\left(x_{j}-\mu_{x}\right)^{2}}{\left(\sigma_{x}^{2}\right)}-\frac{\beta_{1}\left(Y_{1 j}-\alpha_{1}-\beta_{1} \mu_{x}\right)\left(x_{j}-\mu_{x}\right)}{\sigma_{1}^{2}}-\frac{\beta_{2}\left(Y_{2 j}-\alpha_{2}-\beta_{2} \mu_{x}\right)\left(x_{j}-\mu_{x}\right)}{\sigma_{2}^{2}} \cdots-\frac{\beta_{p}\left(Y_{p j}-\alpha_{p}-\beta_{p} \mu_{x}\right)\left(x_{j}-\mu_{x}\right)}{\sigma_{p}^{2}}, \\
& c_{11}=\frac{K\left(x_{j}-\mu_{x}\right)^{2}}{\left(\sigma_{x}^{2}\right)}-\sum_{i=1}^{p} \frac{\beta_{i}\left(Y_{i j}-\alpha_{i}-\beta_{i} \mu_{x}\right)\left(x_{j}-\mu_{x}\right)}{\sigma_{i}^{2}}=\frac{k\left(x_{j}-\mu_{x}\right)^{2}}{\left(\sigma_{x}^{2}\right)}-\left(x_{j}-\mu_{x}\right) \sum_{i=1}^{p} \frac{\beta_{i}\left(Y_{i j}-\alpha_{i}-\beta_{i} \mu_{x}\right)}{\sigma_{i}^{2}}, \\
& c_{22}=-\frac{\beta_{1} B A}{\sigma_{1}^{2}}+\frac{B^{2}}{\sigma_{1}^{2}}=-\frac{\beta_{1}\left(Y_{1 j}-\alpha_{1}-\beta_{1} \mu_{x}\right)\left(\left(x_{j}-\mu_{x}\right)\right.}{\sigma_{1}^{2}}+\frac{\left(Y_{1 j}-\alpha_{1}-\beta_{1} \mu_{x}\right)^{2}}{\sigma_{1}^{2}}, \\
& c_{33}=-\frac{\beta_{2} C A}{\sigma_{2}^{2}}+\frac{C^{2}}{\sigma_{2}^{2}}=-\frac{\beta_{2}\left(Y_{2 j}-\alpha_{2}-\beta_{2} \mu_{x}\right)\left(\left(x_{j}-\mu_{x}\right)\right.}{\sigma_{2}^{2}}+\frac{\left(Y_{2 j}-\alpha_{2}-\beta_{2} \mu_{x}\right)^{2}}{\sigma_{2}^{2}}, \\
& c_{p+1, p+1}=-\frac{\beta_{p} D A}{\sigma_{p}^{2}}+\frac{D^{2}}{\sigma_{p}^{2}}=-\frac{\beta_{p}\left(Y_{p j}-\alpha_{p}-\beta_{p} \mu_{x}\right)\left(\left(x_{j}-\mu_{x}\right)\right.}{\sigma_{p}^{2}}+\frac{\left(Y_{p j}-\alpha_{p}-\beta_{p} \mu_{x}\right)^{2}}{\sigma_{p}^{2}},
\end{aligned}
$$

Em seguida temos:

$$
\begin{aligned}
& \operatorname{tr}(I I . I I I)=c_{11}+c_{22}+c_{33}+\cdots+c_{(p+1)(p+1)} \\
& =\frac{K\left(x_{j}-\mu_{x}\right)^{2}}{\left(\sigma_{x}^{2}\right)}-\left(x_{j}-\mu_{x}\right) \sum_{i=1}^{p} \frac{\beta_{i}\left(Y_{i j}-\alpha_{i}-\beta_{i} \mu_{x}\right)}{\sigma_{i}^{2}}-\frac{\beta_{1}\left(Y_{1 j}-\alpha_{1}-\beta_{1} \mu_{x}\right)\left(x_{j}-\mu_{x}\right)}{\sigma_{1}^{2}}+\frac{\left(Y_{1 j}-\alpha_{1}-\beta_{1} \mu_{x}\right)^{2}}{\sigma_{x}^{2} \sigma_{1}^{2}}+-\frac{\beta_{2}\left(Y_{2 j}-\alpha_{2}-\beta_{2} \mu_{x}\right)\left(x_{j}-\mu_{x}\right)}{\sigma_{2}^{2}}+ \\
& \frac{\left(Y_{2 j}-\alpha_{2}-\beta_{2} \mu_{x}\right)^{2}}{\sigma_{x}^{2} \sigma_{2}^{2}}-\frac{\beta_{p}\left(Y_{p j}-\alpha_{p}-\beta_{p} \mu_{x}\right)\left(x_{j}-\mu_{x}\right)}{\sigma_{p}^{2}}+\frac{\left(Y_{p j}-\alpha_{p}-\beta_{p} \mu_{x}\right)^{2}}{\sigma_{x}^{2} \sigma_{p}^{2}}, \\
& =\frac{K\left(x_{j}-\mu_{x}\right)^{2}}{\left(\sigma_{x}^{2}\right)}-\left(x_{j}-\mu_{x}\right) \sum_{i=1}^{p} \frac{\beta_{i}\left(Y_{i j}-\alpha_{i}-\beta_{i} \mu_{x}\right)}{\sigma_{i}^{2}}-\left(x_{j}-\mu_{x}\right) \sum_{i=1}^{p} \frac{\beta_{i}\left(Y_{i j}-\alpha_{i}-\beta_{i} \mu_{x}\right)}{\sigma_{i}^{2}}+\sum_{i=1}^{p} \frac{\left(Y_{i j}-\alpha_{i}-\beta_{i} \mu_{x}\right)^{2}}{\sigma_{i}^{2}} \\
& =\frac{K\left(x_{j}-\mu_{x}\right)^{2}}{\left(\sigma_{x}^{2}\right)}-2\left(x_{j}-\mu_{x}\right) \sum_{i=1}^{p} \frac{\beta_{i}\left(Y_{i j}-\alpha_{i}-\beta_{i} \mu_{x}\right)}{\sigma_{i}^{2}}+\sum_{i=1}^{p} \frac{\left(Y_{i j}-\alpha_{i}-\beta_{i} \mu_{x}\right)^{2}}{\sigma_{i}^{2}} .
\end{aligned}
$$

Agora, temos: 


$$
=-\operatorname{nln}(2 \pi)-\frac{n}{2} \ln \left(\sigma_{x}^{2} \prod_{i=1}^{p} \sigma_{i}^{2}\right)-\frac{1}{2} \sum_{j=1}^{n}\left[\frac{k\left(x_{j}-\mu_{x}\right)^{2}}{\left(\sigma_{x}^{2}\right)}-2\left(x_{j}-\mu_{x}\right) \sum_{i=1}^{p} \frac{\beta_{i}\left(Y_{i j}-\alpha_{i}-\beta_{i} \mu_{x}\right)}{\sigma_{i}^{2}}+\sum_{i=1}^{p} \frac{\left(Y_{i j}-\alpha_{i}-\beta_{i} \mu_{x}\right)^{2}}{\sigma_{i}^{2}}\right] \text {.(D.3) }
$$

onde:

$A=\left(x_{j}-\mu_{x}\right), B=\left(Y_{1 j}-\alpha_{1}-\beta_{1} \mu_{x}\right), C=\left(Y_{2 j}-\alpha_{2}-\beta_{2} \mu_{x}\right)$,

$D=\left(Y_{p j}-\alpha_{p}-\beta_{p} \mu_{x}\right)$, e $K=1+\sum_{i=1}^{p} \frac{\sigma_{x}^{2} \beta_{i}^{2}}{\sigma_{i}^{2}}$.

Passemos agora a calcular os estimadores para os parâmetros utilizados no algoritmo EM.

Passemos a calcular: $\frac{\partial \ln (L(\theta / Z))}{\partial \mu_{x}}$, mas antes, temos de calcular a derivada dos seguintes termos de $\ln (L(\theta / Z))$ em relação a $\mu_{x}$ :

$\left[-n \ln (2 \pi)-\frac{n}{2} \ln \left(\left|\Sigma_{Z}\right|\right)\right]^{\prime}=0$,

$\left[\Sigma_{z}^{-1}\right]^{\prime}=\left[\left(\begin{array}{ccccc}\frac{k}{\sigma_{x}^{2}} & -\frac{\beta_{1}}{\sigma_{1}^{2}} & -\frac{\beta_{2}}{\sigma_{2}^{2}} & \cdots & -\frac{\beta_{p}}{\sigma_{p}^{2}} \\ -\frac{\beta_{1}}{\sigma_{1}^{2}} & \frac{1}{\sigma_{1}^{2}} & 0 & \cdots & 0 \\ -\frac{\beta_{2}}{\sigma_{2}^{2}} & 0 & \frac{1}{\sigma_{2}^{2}} & \cdots & 0 \\ \vdots & \vdots & \vdots & \ddots & \vdots \\ -\frac{\beta_{p}}{\sigma_{p}^{2}} & 0 & 0 & \cdots & \frac{1}{\sigma_{p}^{2}}\end{array}\right)\right]^{\prime}=\left(\begin{array}{ccccc}0 & 0 & 0 & \cdots & 0 \\ 0 & 0 & 0 & \cdots & 0 \\ 0 & 0 & 0 & \cdots & 0 \\ \vdots & \vdots & \vdots & \ddots & \vdots \\ 0 & 0 & 0 & \cdots & 0\end{array}\right)$,

$\left[\left(Z-\mu_{z}\right)\right]^{\prime}=\left[\left(\begin{array}{c}x_{j}-\mu_{x} \\ Y_{1 j}-\alpha_{1}-\beta_{1} \mu_{x} \\ Y_{2 j}-\alpha_{2}-\beta_{2} \mu_{x} \\ \vdots \\ Y_{p j}-\alpha_{p}-\beta_{p} \mu_{x}\end{array}\right)\right]^{\prime}=\left(\begin{array}{c}-1 \\ -\beta_{1} \\ -\beta_{2} \\ \vdots \\ -\beta_{p}\end{array}\right)$.

Agora, temos:

$$
\begin{aligned}
& \frac{\partial \ln (L(\theta / Z))}{\partial \mu_{x}}=\left[-n \ln \left(2 \Pi|\Sigma|^{\frac{1}{2}}\right)-\frac{1}{2} \sum_{j=1}^{n}\left(Z_{j}-\mu_{Z}\right)^{\prime} \Sigma_{Z}^{-1}\left(Z_{j}-\mu_{Z}\right)\right]^{\prime} \\
& =\left[-n \ln \left(2 \Pi|\Sigma|^{\frac{1}{2}}\right)\right]^{\prime}-\left[\frac{1}{2} \sum_{j=1}^{n}\left(Z_{j}-\mu_{Z}\right)^{\prime} \Sigma_{Z}^{-1}\left(Z_{j}-\mu_{Z}\right)\right]^{\prime}, \\
& =0-\frac{1}{2} \sum_{j=1}^{n}\left[\left(Z_{j}-\mu_{Z}\right)^{\prime} \Sigma_{Z}^{-1}\left(Z_{j}-\mu_{Z}\right)\right]^{\prime} \\
& =-\frac{1}{2} \sum_{j=1}^{n}\left[\left(Z_{j}-\mu_{Z}\right)^{t}\right]^{\prime} \Sigma_{Z}^{-1}\left(Z_{j}-\mu_{Z}\right)+\left(Z_{j}-\mu_{Z}\right)^{t}\left[\Sigma_{Z}^{-1}\left(Z_{j}-\mu_{Z}\right)\right]^{\prime}, \\
& =-\frac{1}{2} \sum_{j=1}^{n}\left[\left(Z_{j}-\mu_{Z}\right)^{t}\right]^{\prime} \Sigma_{Z}^{-1}\left(Z_{j}-\mu_{Z}\right)+\left(Z_{j}-\mu_{Z}\right)^{t}\left[\left(\Sigma_{Z}^{-1}\right)^{\prime}\left(Z_{j}-\mu_{Z}\right)+\left(\Sigma_{Z}^{-1}\right)\left(Z_{j}-\mu_{Z}\right)^{\prime}\right]
\end{aligned}
$$




$$
\begin{aligned}
& =-\frac{1}{2} \sum_{j=1}^{n}\left[\left(Z_{j}-\mu_{Z}\right)^{t}\right]^{\prime} \Sigma_{Z}^{-1}\left(Z_{j}-\mu_{Z}\right)+\left(Z_{j}-\mu_{Z}\right)^{t}\left[\left(\Sigma_{Z}^{-1}\right)\left(Z_{j}-\mu_{Z}\right)^{\prime}\right], \\
& =-\frac{1}{2} \sum_{j=1}^{n} 2\left[\left(Z_{j}-\mu_{Z}\right)^{t}\right]^{\prime} \Sigma_{Z}^{-1}\left(Z_{j}-\mu_{Z}\right) \text {, } \\
& =-\sum_{j=1}^{n}\left[\left(Z_{j}-\mu_{Z}\right)^{t}\right]^{\prime} \Sigma_{Z}^{-1}\left(Z_{j}-\mu_{Z}\right) \\
& =-\sum_{j=1}^{n}\left(\begin{array}{c}
-1 \\
-\beta_{1} \\
-\beta_{2} \\
\vdots \\
-\beta_{p}
\end{array}\right)^{t}\left(\begin{array}{ccccc}
\frac{K}{\sigma_{x}^{2}} & -\frac{\beta_{1}}{\sigma_{1}^{2}} & -\frac{\beta_{2}}{\sigma_{2}^{2}} & \cdots & -\frac{\beta_{p}}{\sigma_{p}^{2}} \\
-\frac{\beta_{1}}{\sigma_{1}^{2}} & \frac{1}{\sigma_{1}^{2}} & 0 & \cdots & 0 \\
-\frac{\beta_{2}}{\sigma_{2}^{2}} & 0 & \frac{1}{\sigma_{2}^{2}} & \cdots & 0 \\
\vdots & \vdots & \vdots & \ddots & \vdots \\
-\frac{\beta_{p}}{\sigma_{p}^{2}} & 0 & 0 & \cdots & \frac{1}{\sigma_{p}^{2}}
\end{array}\right)\left(\begin{array}{c}
x_{j}-\mu_{x} \\
Y_{1 j}-\alpha_{1}-\beta_{1} \mu_{x} \\
Y_{2 j}-\alpha_{2}-\beta_{2} \mu_{x} \\
\vdots \\
Y_{p j}-\alpha_{p}-\beta_{p} \mu_{x}
\end{array}\right) \text {, } \\
& =-\sum_{j=1}^{n}\left(\begin{array}{llllll}
-\frac{K}{\sigma_{x}^{2}}+\sum_{i=1}^{p} \frac{\beta_{i}^{2}}{\sigma_{i}^{2}} & \frac{\beta_{1}}{\sigma_{1}^{2}}-\frac{\beta_{1}}{\sigma_{1}^{2}} & \frac{\beta_{2}}{\sigma_{2}^{2}}-\frac{\beta_{2}}{\sigma_{2}^{2}} & \cdots & \frac{\beta_{p}}{\sigma_{p}^{2}}-\frac{\beta_{p}}{\sigma_{p}^{2}}
\end{array}\right)\left(\begin{array}{c}
x_{j}-\mu_{x} \\
Y_{1 j}-\alpha_{1}-\beta_{1} \mu_{x} \\
Y_{2 j}-\alpha_{2}-\beta_{2} \mu_{x} \\
\vdots \\
Y_{p j}-\alpha_{p}-\beta_{p} \mu_{x}
\end{array}\right), \\
& =-\sum_{j=1}^{n}\left(\begin{array}{lllll}
-\frac{K}{\sigma_{x}^{2}}+\sum_{i=1}^{p} \frac{\beta_{i}^{2}}{\sigma_{i}^{2}} & 0 & 0 & \cdots & 0
\end{array}\right)\left(\begin{array}{c}
x_{j}-\mu_{x} \\
Y_{1 j}-\alpha_{1}-\beta_{1} \mu_{x} \\
Y_{2 j}-\alpha_{2}-\beta_{2} \mu_{x} \\
\vdots \\
Y_{p j}-\alpha_{p}-\beta_{p} \mu_{x}
\end{array}\right) \text {, } \\
& =-\sum_{j=1}^{n}\left[-\frac{K}{\sigma_{x}^{2}}\left(x_{j}-\mu_{x}\right)+\sum_{i=1}^{p} \frac{\beta_{i}^{2}}{\sigma_{i}^{2}}\left(x_{j}-\mu_{x}\right)\right] \text {, } \\
& =-\sum_{j=1}^{n}\left[\left(x_{j}-\mu_{x}\right)\left(-\frac{K}{\sigma_{x}^{2}}+\sum_{i=1}^{p} \frac{\beta_{i}^{2}}{\sigma_{i}^{2}}\right)\right] \text {, } \\
& =-\left(-\frac{K}{\sigma_{x}^{2}}+\sum_{i=1}^{p} \frac{\beta_{i}^{2}}{\sigma_{i}^{2}}\right) \sum_{j=1}^{n}\left(x_{j}-\mu_{x}\right) \text {, } \\
& =-\left(-\frac{K}{\sigma_{x}^{2}}+\sum_{i=1}^{p} \frac{\beta_{i}^{2}}{\sigma_{i}^{2}}\right)\left(\sum_{j=1}^{n} x_{j}-\sum_{j=1}^{n} \mu_{x}\right) \text {, } \\
& =-\left(-\frac{K}{\sigma_{x}^{2}}+\sum_{i=1}^{p} \frac{\beta_{i}^{2}}{\sigma_{i}^{2}}\right)\left(n \bar{x}-n \mu_{x}\right) \text {, }
\end{aligned}
$$

que igualando a zero temos:

$-\left(-\frac{K}{\sigma_{x}^{2}}+\sum_{i=1}^{p} \frac{\beta_{i}^{2}}{\sigma_{i}^{2}}\right)\left(n \bar{x}-n \mu_{x}\right)=0 \Rightarrow\left(n \bar{x}-n \mu_{x}\right)=0 \Rightarrow n \bar{x}=n \mu_{x} \Rightarrow \mu_{x}=\bar{x}$.

Portanto, temos: 


$$
\hat{\mu}_{x}=\bar{x}
$$

Agora, passemos a calcular: $\frac{\partial \ln (L(\theta / Z))}{\partial \alpha_{i}}$, mas antes, temos de calcular a derivada dos seguintes termos de $\ln (L(\theta / Z))$ em relação a $\partial \alpha_{i}$ :

$$
\begin{gathered}
{\left[\left(Z-\mu_{z}\right)\right]^{\prime}=\left[\left(\begin{array}{c}
x_{j}-\mu_{x} \\
Y_{1 j}-\alpha_{1}-\beta_{1} \mu_{x} \\
Y_{2 j}-\alpha_{2}-\beta_{2} \mu_{x} \\
\vdots \\
Y_{p j}-\alpha_{p}-\beta_{p} \mu_{x}
\end{array}\right)\right]^{\prime}=\left(\begin{array}{c}
0 \\
0 \\
-1 \\
\vdots \\
0
\end{array}\right),} \\
{\left[-n \ln (2 \pi)-\frac{n}{2} \ln \left(\left|\Sigma_{Z}\right|\right)\right]^{\prime}=0,} \\
{\left[\Sigma_{z}^{-1}\right]^{\prime}=\left[\left(\begin{array}{ccccc}
\frac{k}{\sigma_{x}^{2}} & -\frac{\beta_{1}}{\sigma_{1}^{2}} & -\frac{\beta_{2}}{\sigma_{2}^{2}} & \cdots & -\frac{\beta_{p}}{\sigma_{p}^{2}} \\
-\frac{\beta_{1}}{\sigma_{1}^{2}} & \frac{1}{\sigma_{1}^{2}} & 0 & \cdots & 0 \\
-\frac{\beta_{2}}{\sigma_{2}^{2}} & 0 & \frac{1}{\sigma_{2}^{2}} & \cdots & 0 \\
\vdots & \vdots & \vdots & \ddots & \vdots \\
-\frac{\beta_{p}}{\sigma_{p}^{2}} & 0 & 0 & \cdots & \frac{1}{\sigma_{p}^{2}}
\end{array}\right)\right]^{\prime}=\left(\begin{array}{ccccc}
0 & 0 & 0 & \cdots & 0 \\
0 & 0 & 0 & \cdots & 0 \\
0 & 0 & 0 & \cdots & 0 \\
\vdots & \vdots & \vdots & \ddots & \vdots \\
0 & 0 & 0 & \cdots & 0
\end{array}\right) .}
\end{gathered}
$$

Agora temos:

$$
\begin{aligned}
& \frac{\partial \ln (L(\theta / Z))}{\partial \alpha_{i}}=\left[-n \ln \left(2 \Pi|\Sigma|^{\frac{1}{2}}\right)-\frac{1}{2} \sum_{j=1}^{n}\left(Z_{j}-\mu_{Z}\right)^{\prime} \Sigma_{Z}^{-1}\left(Z_{j}-\mu_{Z}\right)\right]^{\prime}, \\
& =\left[-n \ln \left(2 \Pi|\Sigma|^{\frac{1}{2}}\right)\right]^{\prime}-\left[\frac{1}{2} \sum_{j=1}^{n}\left(Z_{j}-\mu_{Z}\right)^{\prime} \Sigma_{Z}^{-1}\left(Z_{j}-\mu_{Z}\right)\right]^{\prime}, \\
& =0-\frac{1}{2} \sum_{j=1}^{n}\left[\left(Z_{j}-\mu_{Z}\right)^{\prime} \Sigma_{Z}^{-1}\left(Z_{j}-\mu_{Z}\right)\right]^{\prime} \\
& =-\frac{1}{2} \sum_{j=1}^{n}\left[\left(Z_{j}-\mu_{Z}\right)^{t}\right]^{\prime} \Sigma_{Z}^{-1}\left(Z_{j}-\mu_{Z}\right)+\left(Z_{j}-\mu_{Z}\right)^{t}\left[\Sigma_{Z}^{-1}\left(Z_{j}-\mu_{Z}\right)\right]^{\prime}, \\
& =-\frac{1}{2} \sum_{j=1}^{n}\left[\left(Z_{j}-\mu_{Z}\right)^{t}\right]^{\prime} \Sigma_{Z}^{-1}\left(Z_{j}-\mu_{Z}\right)+\left(Z_{j}-\mu_{Z}\right)^{t}\left[\left(\Sigma_{Z}^{-1}\right)^{\prime}\left(Z_{j}-\mu_{Z}\right)+\left(\Sigma_{Z}^{-1}\right)\left(Z_{j}-\mu_{Z}\right)^{\prime}\right] \\
& =-\frac{1}{2} \sum_{j=1}^{n}\left[\left(Z_{j}-\mu_{Z}\right)^{t}\right]^{\prime} \Sigma_{Z}^{-1}\left(Z_{j}-\mu_{Z}\right)+\left(Z_{j}-\mu_{Z}\right)^{t}\left[\left(\Sigma_{Z}^{-1}\right)\left(Z_{j}-\mu_{Z}\right)^{\prime}\right] \\
& =-\frac{1}{2} \sum_{j=1}^{n} 2\left[\left(Z_{j}-\mu_{Z}\right)^{t}\right]^{\prime} \Sigma_{Z}^{-1}\left(Z_{j}-\mu_{Z}\right) \\
& =-\sum_{j=1}^{n}\left[\left(Z_{j}-\mu_{Z}\right)^{t}\right]^{\prime} \Sigma_{Z}^{-1}\left(Z_{j}-\mu_{Z}\right)
\end{aligned}
$$




$$
\begin{aligned}
& =-\sum_{j=1}^{n}\left(\begin{array}{c}
0 \\
0 \\
-1 \\
\vdots \\
0
\end{array}\right)^{t}\left(\begin{array}{ccccc}
\frac{k}{\sigma_{x_{1}}^{2}} & -\frac{\beta_{1}}{\sigma_{1}^{2}} & -\frac{\beta_{2}}{\sigma_{2}^{2}} & \cdots & -\frac{\beta_{p}}{\sigma_{p}^{2}} \\
-\frac{\beta_{1}}{\sigma_{1}^{2}} & \frac{1}{\sigma_{1}^{2}} & 0 & \cdots & 0 \\
-\frac{\beta_{2}}{\sigma_{2}^{2}} & 0 & \frac{1}{\sigma_{2}^{2}} & \cdots & 0 \\
\vdots & \vdots & \vdots & \ddots & \vdots \\
-\frac{\beta_{p}}{\sigma_{p}^{2}} & 0 & 0 & \cdots & \frac{1}{\sigma_{p}^{2}}
\end{array}\right)\left(\begin{array}{c}
x_{j}-\mu_{x} \\
Y_{1 j}-\alpha_{1}-\beta_{1} \mu_{x} \\
Y_{2 j}-\alpha_{2}-\beta_{2} \mu_{x} \\
\vdots \\
Y_{p j}-\alpha_{p}-\beta_{p} \mu_{x}
\end{array}\right), \\
& =-\sum_{j=1}^{n}\left(\begin{array}{lllll}
\frac{\beta_{2}}{\sigma_{2}^{2}} & 0 & -\frac{1}{\sigma_{2}^{2}} & \cdots & 0
\end{array}\right)\left(\begin{array}{c}
x_{j}-\mu_{x} \\
Y_{1 j}-\alpha_{1}-\beta_{1} \mu_{x} \\
Y_{2 j}-\alpha_{2}-\beta_{2} \mu_{x} \\
\vdots \\
Y_{p j}-\alpha_{p}-\beta_{p} \mu_{x}
\end{array}\right) \text {, } \\
& =-\sum_{j=1}^{n} \frac{\beta_{2}\left(x_{j}-\mu_{x}\right)}{\sigma_{2}^{2}}-\frac{\left(Y_{2 j}-\alpha_{2}-\beta_{2} \mu_{x}\right)}{\sigma_{2}^{2}} \text {, } \\
& =-\frac{1}{\sigma_{2}^{2}} \sum_{j=1}^{n}\left[\beta_{2}\left(x_{j}-\mu_{x}\right)-\left(Y_{2 j}-\alpha_{2}-\beta_{2} \mu_{x}\right)\right], \\
& =-\frac{1}{\sigma_{2}^{2}}\left[\beta_{2} \sum_{j=1}^{n}\left(x_{j}-\mu_{x}\right)-\sum_{j=1}^{n}\left(Y_{2 j}-\alpha_{2}-\beta_{2} \mu_{x}\right)\right] \text {, } \\
& =-\frac{1}{\sigma_{2}^{2}}\left[\beta_{2}(n \bar{x}-n \bar{x})-\left(n \bar{Y}_{2}-n \alpha_{2}-n \beta_{2} n \bar{x}\right)\right], \\
& =-\frac{1}{\sigma_{2}^{2}}\left[-\left(n \bar{Y}_{2}-n \alpha_{2}-n \beta_{2} n \bar{x}\right)\right] \text {. }
\end{aligned}
$$

Igualando $\frac{\partial \ln (L(\theta / Z))}{\partial \alpha_{i}}$ a zero temos:

$=-\left(n \bar{Y}_{2}-n \alpha_{2}-n \beta_{2} n \bar{x}\right)=0$

$=-n\left(\bar{Y}_{2}-\alpha_{2}-\beta_{2} \bar{x}\right)=0$,

$=\left(\bar{Y}_{2}-\alpha_{2}-\beta_{2} \bar{x}\right)=0$, que fica: $\hat{\alpha}_{2}=\bar{Y}_{2}-\beta_{2} \bar{x}$

que geralizando temos:

$$
\hat{\alpha}_{i}=\bar{Y}_{i}-\beta_{i} \bar{x}
$$

Agora, passemos a calcular: $\frac{\partial \ln (L(\theta / Z))}{\partial \beta_{i}}$, mas antes, temos de calcular a derivada dos seguintes termos de $\ln (L(\theta / Z))$ em relação a $\partial \beta_{i}$ : 


$$
\left[\left(Z-\mu_{z}\right)\right]^{\prime}=\left[\left(\begin{array}{c}
x_{j}-\mu_{x} \\
Y_{1 j}-\alpha_{1}-\beta_{1} \mu_{x} \\
Y_{2 j}-\alpha_{2}-\beta_{2} \mu_{x} \\
\vdots \\
Y_{p j}-\alpha_{p}-\beta_{p} \mu_{x}
\end{array}\right)\right]^{\prime}=\left(\begin{array}{c}
0 \\
0 \\
-\mu_{x} \\
\vdots \\
0
\end{array}\right)
$$

$\left[-n \ln (2 \pi)-\frac{n}{2} \ln \left(\left|\Sigma_{Z}\right|\right)\right]^{\prime}=0$,

$$
\begin{gathered}
\left(\frac{K}{\sigma_{x}^{2}}\right)^{\prime}=\left(\frac{\left.1+\sigma_{x}^{2} \sum_{i=1}^{p} \frac{\beta_{i}^{2}}{\sigma_{x}^{2}}\right)^{\prime}}{\sigma^{2}}=\left(\frac{1}{\sigma_{x}^{2}}+\sum_{i=1}^{p} \frac{\beta_{i}^{2}}{\sigma_{i}^{2}}\right)^{\prime}=\frac{2 \beta_{i}}{\sigma_{i}^{2}},\right. \\
\left.\Sigma_{z}^{-1}\right]^{\prime}=\left[\left(\begin{array}{ccccc}
\frac{K}{\sigma_{x}^{2}} & -\frac{\beta_{1}}{\sigma_{1}^{2}} & -\frac{\beta_{2}}{\sigma_{2}^{2}} & \cdots & -\frac{\beta_{p}}{\sigma_{p}^{2}} \\
-\frac{\beta_{1}}{\sigma_{1}^{2}} & \frac{1}{\sigma_{1}^{2}} & 0 & \cdots & 0 \\
-\frac{\beta_{2}}{\sigma_{2}^{2}} & 0 & \frac{1}{\sigma_{2}^{2}} & \cdots & 0 \\
\vdots & \vdots & \vdots & \ddots & \vdots \\
-\frac{\beta_{p}}{\sigma_{p}^{2}} & 0 & 0 & \cdots & \frac{1}{\sigma_{p}^{2}}
\end{array}\right)\right]^{\prime}=\left(\begin{array}{cccccc}
\frac{2 \beta_{2}}{\sigma_{2}^{2}} & 0 & -\frac{1}{\sigma_{2}^{2}} & \cdots & 0 \\
0 & 0 & 0 & \cdots & 0 \\
-\frac{1}{\sigma_{2}^{2}} & 0 & 0 & \cdots & 0 \\
\vdots & \vdots & \vdots & \ddots & \vdots \\
0 & 0 & 0 & \cdots & 0
\end{array}\right) .
\end{gathered}
$$

Agora, temos:

$$
\begin{aligned}
& \frac{\partial \ln (L(\theta / Z))}{\partial \beta_{i}}=\left[-n \ln \left(2 \Pi|\Sigma|^{\frac{1}{2}}\right)-\frac{1}{2} \sum_{j=1}^{n}\left(Z_{j}-\mu_{Z}\right)^{\prime} \Sigma_{Z}^{-1}\left(Z_{j}-\mu_{Z}\right)\right]^{\prime}, \\
& =\left[-n \ln \left(2 \Pi|\Sigma|^{\frac{1}{2}}\right)\right]^{\prime}-\left[\frac{1}{2} \sum_{j=1}^{n}\left(Z_{j}-\mu_{Z}\right)^{\prime} \Sigma_{Z}^{-1}\left(Z_{j}-\mu_{Z}\right)\right]^{\prime}, \\
& =0-\frac{1}{2} \sum_{j=1}^{n}\left[\left(Z_{j}-\mu_{Z}\right)^{\prime} \Sigma_{Z}^{-1}\left(Z_{j}-\mu_{Z}\right)\right]^{\prime}, \\
& =-\frac{1}{2} \sum_{j=1}^{n}\left[\left(Z_{j}-\mu_{Z}\right)^{t}\right]^{\prime} \Sigma_{Z}^{-1}\left(Z_{j}-\mu_{Z}\right)+\left(Z_{j}-\mu_{Z}\right)^{t}\left[\Sigma_{Z}^{-1}\left(Z_{j}-\mu_{Z}\right)\right]^{\prime}, \\
& =-\frac{1}{2} \sum_{j=1}^{n}\left[\left(Z_{j}-\mu_{Z}\right)^{t}\right]^{\prime} \Sigma_{Z}^{-1}\left(Z_{j}-\mu_{Z}\right)+\left(Z_{j}-\mu_{Z}\right)^{t}\left[\left(\Sigma_{Z}^{-1}\right)^{\prime}\left(Z_{j}-\mu_{Z}\right)+\left(\Sigma_{Z}^{-1}\right)\left(Z_{j}-\mu_{Z}\right)^{\prime}\right] \text {, } \\
& =-\frac{1}{2} \sum_{j=1}^{n}\left[\left(z_{j}-\mu_{Z}\right)^{t}\right]^{\prime} \Sigma_{Z}^{-1}\left(z_{j}-\mu_{Z}\right)+\left(z_{j}-\mu_{Z}\right)^{t}\left(\Sigma_{Z}^{-1}\right)^{\prime}\left(z_{j}-\mu_{Z}\right)+\left(z_{j}-\mu_{Z}\right)^{t}\left[\left(\Sigma_{Z}^{-1}\right)\left(z_{j}-\mu_{Z}\right)^{\prime}\right], \\
& =-\frac{1}{2} \sum_{j=1}^{n} 2\left[\left(Z_{j}-\mu_{Z}\right)^{t}\right]^{\prime} \Sigma_{Z}^{-1}\left(Z_{j}-\mu_{Z}\right)+\left(Z_{j}-\mu_{Z}\right)^{t}\left[\left(\Sigma_{Z}^{-1}\right)^{\prime}\left(Z_{j}-\mu_{Z}\right),\right. \\
& =-\frac{1}{2} \sum_{j=1}^{n} 2\left(\begin{array}{c}
0 \\
0 \\
-\mu_{x} \\
\vdots \\
0
\end{array}\right)^{t}\left(\begin{array}{ccccc}
\frac{K}{\sigma_{\pi}^{2}} & -\frac{\beta_{1}}{\sigma_{1}^{2}} & -\frac{\beta_{2}}{\sigma_{2}^{2}} & \cdots & -\frac{\beta_{p}}{\sigma_{p}^{2}} \\
-\frac{\beta_{1}}{\sigma_{1}^{2}} & \frac{1}{\sigma_{1}^{2}} & 0 & \cdots & 0 \\
-\frac{\beta_{2}}{\sigma_{2}^{2}} & 0 & \frac{1}{\sigma_{2}^{2}} & \cdots & 0 \\
\vdots & \vdots & \vdots & \ddots & \vdots \\
-\frac{\beta_{p}}{\sigma_{p}^{2}} & 0 & 0 & \cdots & \frac{1}{\sigma_{p}^{2}}
\end{array}\right)\left(\begin{array}{c}
x_{j}-\mu_{x} \\
Y_{1 j}-\alpha_{1}-\beta_{1} \mu_{x} \\
Y_{2 j}-\alpha_{2}-\beta_{2} \mu_{x} \\
\vdots \\
Y_{p j}-\alpha_{p}-\beta_{p} \mu_{x}
\end{array}\right)+
\end{aligned}
$$




$$
\begin{aligned}
& \left(\begin{array}{c}
x_{j}-\mu_{x} \\
Y_{1 j}-\alpha_{1}-\beta_{1} \mu_{x} \\
Y_{2 j}-\alpha_{2}-\beta_{2} \mu_{x} \\
\vdots \\
Y_{p j}-\alpha_{p}-\beta_{p} \mu_{x}
\end{array}\right)^{t}\left(\begin{array}{ccccc}
\frac{2 \beta_{2}}{\sigma_{2}^{2}} & 0 & -\frac{1}{\sigma_{2}^{2}} & \cdots & 0 \\
0 & 0 & 0 & \cdots & 0 \\
-\frac{1}{\sigma_{2}^{2}} & 0 & 0 & \cdots & 0 \\
\vdots & \vdots & \vdots & \ddots & \vdots \\
0 & 0 & 0 & \cdots & 0
\end{array}\right)\left(\begin{array}{c}
x_{j}-\mu_{x} \\
Y_{1 j}-\alpha_{1}-\beta_{1} \mu_{x} \\
Y_{2 j}-\alpha_{2}-\beta_{2} \mu_{x} \\
\vdots \\
Y_{p j}-\alpha_{p}-\beta_{p} \mu_{x}
\end{array}\right) \\
& =-\sum_{j=1}^{n} 2\left(\begin{array}{ccccc}
\frac{\mu_{x} \beta_{2}}{\sigma_{2}^{2}} & 0 & -\frac{\mu_{x}}{\sigma_{2}^{2}} & \cdots & 0
\end{array}\right)\left(\begin{array}{c}
x_{j}-\mu_{x} \\
Y_{1 j}-\alpha_{1}-\beta_{1} \mu_{x} \\
Y_{2 j}-\alpha_{2}-\beta_{2} \mu_{x} \\
\vdots \\
Y_{p j}-\alpha_{p}-\beta_{p} \mu_{x}
\end{array}\right)+ \\
& \left(\begin{array}{lllll}
\frac{\left(x_{j}-\mu_{x}\right) \beta_{2}}{\sigma_{2}^{2}}-\frac{\left(Y_{2 j}-\alpha_{2}-\beta_{2} \mu_{x}\right)}{\sigma_{2}^{2}} & 0 & -\frac{\left(x_{j}-\mu_{x}\right)}{\sigma_{2}^{2}} & \cdots & 0
\end{array}\right)\left(\begin{array}{c}
x_{j}-\mu_{x} \\
Y_{1 j}-\alpha_{1}-\beta_{1} \mu_{x} \\
Y_{2 j}-\alpha_{2}-\beta_{2} \mu_{x} \\
\vdots \\
Y_{p j}-\alpha_{p}-\beta_{p} \mu_{x}
\end{array}\right) \text {, } \\
& =-\sum_{j=1}^{n} 2 \frac{\mu_{x} \beta_{2}\left(x_{j}-\mu_{x}\right)}{\sigma_{2}^{2}}+\frac{2 \mu_{x}\left(Y_{2 j}-\alpha_{2}-\beta_{2} \mu_{x}\right)}{\sigma_{2}^{2}}+\frac{\left(x_{j}-\mu_{x}\right)^{2} \beta_{2}}{\sigma_{2}^{2}}-\frac{\left(x_{j}-\mu_{x}\right)\left(Y_{2 j}-\alpha_{2}-\beta_{2} \mu_{x}\right)}{\sigma_{2}^{2}}-\frac{\left(x_{j}-\mu_{x}\right)\left(Y_{2 j}-\alpha_{2}-\beta_{2} \mu_{x}\right)}{\sigma_{2}^{2}}, \\
& =-\sum_{j=1}^{n}-2 \frac{\mu_{x}\left(Y_{2 j}-\alpha_{2}-\beta_{2} \mu_{x}\right)}{\sigma_{2}^{2}}+\frac{2\left(x_{j}-\mu_{x}\right)^{2} \beta_{2}}{\sigma_{2}^{2}}-\frac{2\left(x_{j}-\mu_{x}\right)\left(Y_{2 j}-\alpha_{2}-\beta_{2} \mu_{x}\right)}{\sigma_{2}^{2}} \text {, } \\
& =\sum_{j=1}^{n} 2 \frac{\mu_{x}\left(Y_{2 j}-\alpha_{2}-\beta_{2} \mu_{x}\right)}{\sigma_{2}^{2}}-\frac{2\left(x_{j}-\mu_{x}\right)^{2} \beta_{2}}{\sigma_{2}^{2}}+\frac{2\left(x_{j}-\mu_{x}\right)\left(Y_{2 j}-\alpha_{2}-\beta_{2} \mu_{x}\right)}{\sigma_{2}^{2}} \\
& =\frac{2}{\sigma_{2}^{2}}\left[\sum_{j=1}^{n} \mu_{x}\left(Y_{2 j}-\alpha_{2}-\beta_{2} \mu_{x}\right)-\left(x_{j}-\mu_{x}\right)^{2} \beta_{2}+\left(x_{j}-\mu_{x}\right)\left(Y_{2 j}-\alpha_{2}-\beta_{2} \mu_{x}\right)\right],
\end{aligned}
$$

Tomando $\frac{\partial \ln (L(\theta / Z))}{\partial \beta_{i}}=0$, temos:

$\frac{2}{\sigma_{2}^{2}}\left[\sum_{j=1}^{n} \mu_{x}\left(Y_{2 j}-\alpha_{2}-\beta_{2} \mu_{x}\right)-\left(x_{j}-\mu_{x}\right)^{2} \beta_{2}+\left(x_{j}-\mu_{x}\right)\left(Y_{2 j}-\alpha_{2}-\beta_{2} \mu_{x}\right)\right]=0$,

$\sum_{j=1}^{n} \mu_{x}\left(Y_{2 j}-\alpha_{2}-\beta_{2} \mu_{x}\right)-\left(x_{j}-\mu_{x}\right)^{2} \beta_{2}+\left(x_{j}-\mu_{x}\right)\left(Y_{2 j}-\alpha_{2}-\beta_{2} \mu_{x}\right)=0$,

$\mu_{x} \sum_{j=1}^{n}\left(Y_{2 j}-\alpha_{2}-\beta_{2} \mu_{x}\right)-\beta_{2} \sum_{j=1}^{n}\left(x_{j}-\mu_{x}\right)^{2}+\sum_{j=1}^{n}\left(x_{j}-\mu_{x}\right)\left(Y_{2 j}-\alpha_{2}-\beta_{2} \mu_{x}\right)=0$,

$\mu_{x} \sum_{j=1}^{n}\left(Y_{2 j}-\alpha_{2}-\beta_{2} \mu_{x}\right)+\sum_{j=1}^{n}\left(x_{j}-\mu_{x}\right)\left(Y_{2 j}-\alpha_{2}-\beta_{2} \mu_{x}\right)=\beta_{2} \sum_{j=1}^{n}\left(x_{j}-\mu_{x}\right)^{2}$.

Sabemos que: $\hat{\alpha}_{i}=\bar{Y}_{i}-\beta_{i} \bar{x}$ e que $\hat{\mu}_{x}=\bar{x}$ então:

$\bar{x} \sum_{j=1}^{n}\left(Y_{2 j}-\left(\bar{Y}_{i}-\beta_{i} \bar{x}\right)-\beta_{2} \bar{x}\right)+\sum_{j=1}^{n}\left(x_{j}-\bar{x}\right)\left(Y_{2 j}-\left(\bar{Y}_{i}-\beta_{i} \bar{x}\right)-\beta_{2} \bar{x}\right)=\beta_{2} \sum_{j=1}^{n}\left(x_{j}-\bar{x}\right)^{2}$, 


$$
\begin{aligned}
& \bar{x} \sum_{j=1}^{n}\left(Y_{2 j}-\bar{Y}_{2}+\beta_{2} \bar{x}-\beta_{2} \bar{x}\right)+\sum_{j=1}^{n}\left(x_{j}-\bar{x}\right)\left(Y_{2 j}-\bar{Y}_{2}+\beta_{2} \bar{x}-\beta_{2} \bar{x}\right)=\beta_{2} \sum_{j=1}^{n}\left(x_{j}-\bar{x}\right)^{2}, \\
& \bar{x} \sum_{j=1}^{n}\left(Y_{2 j}-\bar{Y}_{2}\right)+\sum_{j=1}^{n}\left(x_{j}-\bar{x}\right)\left(Y_{2 j}-\bar{Y}_{2}\right)=\beta_{2} \sum_{j=1}^{n}\left(x_{j}-\bar{x}\right)^{2} \\
& \sum_{j=1}^{n}\left(x_{j}-\bar{x}\right)\left(Y_{2 j}-\bar{Y}_{2}\right)=\beta_{2} \sum_{j=1}^{n}\left(x_{j}-\bar{x}\right)^{2} .
\end{aligned}
$$

Assim temos:

$$
\begin{aligned}
& \beta_{2} \sum_{j=1}^{n}\left(x_{j}-\bar{x}\right)^{2}=\sum_{j=1}^{n}\left(x_{j}-\bar{x}\right)\left(Y_{2 j}-\bar{Y}_{2}\right), \\
& \hat{\beta}_{2}=\frac{\sum_{j=1}^{n}\left(x_{j}-\bar{x}\right)\left(Y_{2 j}-\bar{Y}_{2}\right)}{\sum_{j=1}^{n}\left(x_{j}-\bar{x}\right)^{2}},
\end{aligned}
$$

que generalizando temos:

$$
\hat{\beta}_{i}=\frac{\sum_{j=1}^{n}\left(x_{j}-\bar{x}\right)\left(Y_{i j}-\bar{Y}_{i}\right)}{\sum_{j=1}^{n}\left(x_{j}-\bar{x}\right)^{2}} .
$$

Agora, passemos a calcular: $\frac{\partial \ln (L(\theta / Z))}{\partial \sigma_{x}^{2}}$, mas antes, temos de calcular a derivada dos seguintes termos de $\ln (L(\theta / Z))$ em relação a $\partial \sigma_{x}^{2}$ :

$$
\begin{gathered}
{\left[\left(Z-\mu_{z}\right)\right]^{\prime}=\left[\left(\begin{array}{c}
x_{j}-\mu_{x} \\
Y_{1 j}-\alpha_{1}-\beta_{1} \mu_{x} \\
Y_{2 j}-\alpha_{2}-\beta_{2} \mu_{x} \\
\vdots \\
Y_{p j}-\alpha_{p}-\beta_{p} \mu_{x}
\end{array}\right)\right]^{\prime}=\left(\begin{array}{c}
0 \\
0 \\
0 \\
\vdots \\
0
\end{array}\right),} \\
{\left[-n \ln (2 \pi)-\frac{n}{2} \ln \left(\left|\Sigma_{Z}\right|\right)\right]^{\prime}=\left[-\frac{n}{2} \ln \left(\sigma_{x}^{2} \prod_{i=1}^{p} \sigma_{i}^{2}\right)\right]^{\prime},} \\
=-\frac{n}{2} \frac{\left(\sigma_{x}^{2} \prod_{i=1}^{p} \sigma_{i}^{2}\right)^{\prime}}{\left(\sigma_{x}^{2} \prod_{i=1}^{p} \sigma_{i}^{2}\right)}=-\frac{n}{2} \frac{\left(\prod_{i=1}^{p} \sigma_{i}^{2}\right)}{\left(\sigma_{x}^{2} \prod_{i=1}^{p} \sigma_{i}^{2}\right)}=-\frac{n}{2} \frac{1}{\left(\sigma_{x}^{2}\right)},
\end{gathered}
$$




$$
\begin{aligned}
& \left(\frac{K}{\sigma_{x}^{2}}\right)^{\prime}=\left(\frac{1+\sigma_{x}^{2} \sum_{i=1}^{p} \frac{\beta_{i}^{2}}{\sigma_{i}^{2}}}{\sigma_{x}^{2}}\right)^{\prime}=\left(\frac{1}{\sigma_{x}^{2}}+\sum_{i=1}^{p} \frac{\beta_{i}^{2}}{\sigma_{i}^{2}}\right)^{\prime}=-\frac{1}{\left(\sigma_{x}^{2}\right)^{2}}, \\
& {\left[\Sigma_{z}^{-1}\right]^{\prime}=\left[\left(\begin{array}{ccccc}
\frac{K}{\sigma_{x}^{2}} & -\frac{\beta_{1}}{\sigma_{1}^{2}} & -\frac{\beta_{2}}{\sigma_{2}^{2}} & \cdots & -\frac{\beta_{p}}{\sigma_{p}^{2}} \\
-\frac{\beta_{1}}{\sigma_{1}^{2}} & \frac{1}{\sigma_{1}^{2}} & 0 & \cdots & 0 \\
-\frac{\beta_{2}}{\sigma_{2}^{2}} & 0 & \frac{1}{\sigma_{2}^{2}} & \cdots & 0 \\
\vdots & \vdots & \vdots & \ddots & \vdots \\
-\frac{\beta_{p}}{\sigma_{p}^{2}} & 0 & 0 & \cdots & \frac{1}{\sigma_{p}^{2}}
\end{array}\right)\right]^{\prime}=\left(\begin{array}{ccccc}
-\frac{1}{\left(\sigma_{x}^{2}\right)^{2}} & 0 & 0 & \cdots & 0 \\
0 & 0 & 0 & \cdots & 0 \\
0 & 0 & 0 & \cdots & 0 \\
\vdots & \vdots & \vdots & \ddots & \vdots \\
0 & 0 & 0 & \cdots & 0
\end{array}\right) .}
\end{aligned}
$$

Agora temos:

$$
\begin{aligned}
& \frac{\partial \ln (L(\theta / Z))}{\partial \sigma_{x}}=\left[-n \ln \left(2 \Pi|\Sigma|^{\frac{1}{2}}\right)-\frac{1}{2} \sum_{j=1}^{n}\left(Z_{j}-\mu_{Z}\right)^{\prime} \Sigma_{Z}^{-1}\left(Z_{j}-\mu_{Z}\right)\right]^{\prime}, \\
& =\left[-n \ln \left(2 \Pi|\Sigma|^{\frac{1}{2}}\right)\right]^{\prime}-\left[\frac{1}{2} \sum_{j=1}^{n}\left(Z_{j}-\mu_{Z}\right)^{\prime} \Sigma_{Z}^{-1}\left(Z_{j}-\mu_{Z}\right)\right]^{\prime}, \\
& =-\frac{n}{2} \frac{1}{\left(\sigma_{x}^{2}\right)}-\frac{1}{2} \sum_{j=1}^{n}\left[\left(Z_{j}-\mu_{Z}\right)^{\prime} \Sigma_{Z}^{-1}\left(Z_{j}-\mu_{Z}\right)\right]^{\prime}, \\
& =-\frac{n}{2} \frac{1}{\left(\sigma_{x}^{2}\right)}-\frac{1}{2} \sum_{j=1}^{n}\left[\left(Z_{j}-\mu_{Z}\right)^{t}\right]^{\prime} \Sigma_{Z}^{-1}\left(Z_{j}-\mu_{Z}\right)+\left(Z_{j}-\mu_{Z}\right)^{t}\left[\Sigma_{Z}^{-1}\left(Z_{j}-\mu_{Z}\right)\right]^{\prime} \text {, } \\
& =-\frac{n}{2} \frac{1}{\left(\sigma_{x}^{2}\right)}-\frac{1}{2} \sum_{j=1}^{n}\left(Z_{j}-\mu_{Z}\right)^{t}\left[\left(\Sigma_{Z}^{-1}\right)^{\prime}\left(Z_{j}-\mu_{Z}\right)\right. \text {, } \\
& =-\frac{n}{2} \frac{1}{\left(\sigma_{x}^{2}\right)}-\frac{1}{2} \sum_{j=1}^{n}\left(\begin{array}{c}
x_{j}-\mu_{x} \\
Y_{1 j}-\alpha_{1}-\beta_{1} \mu_{x} \\
Y_{2 j}-\alpha_{2}-\beta_{2} \mu_{x} \\
\vdots \\
Y_{p j}-\alpha_{p}-\beta_{p} \mu_{x}
\end{array}\right)^{t}\left(\begin{array}{ccccc}
-\frac{1}{\left(\sigma_{x}^{2}\right)^{2}} & 0 & 0 & \cdots & 0 \\
0 & 0 & 0 & \cdots & 0 \\
0 & 0 & 0 & \cdots & 0 \\
\vdots & \vdots & \vdots & \ddots & \vdots \\
0 & 0 & 0 & \cdots & 0
\end{array}\right)\left(\begin{array}{c}
x_{j}-\mu_{x} \\
Y_{1 j}-\alpha_{1}-\beta_{1} \mu_{x} \\
Y_{2 j}-\alpha_{2}-\beta_{2} \mu_{x} \\
\vdots \\
Y_{p j}-\alpha_{p}-\beta_{p} \mu_{x}
\end{array}\right) \text {, } \\
& =-\frac{n}{2} \frac{1}{\left(\sigma_{x}^{2}\right)}-\frac{1}{2} \sum_{j=1}^{n}\left(\begin{array}{ccccc}
-\frac{\left(x_{j}-\mu_{x}\right)}{\left(\sigma_{x}^{2}\right)^{2}} & 0 & 0 & \cdots & 0
\end{array}\right)\left(\begin{array}{c}
x_{j}-\mu_{x} \\
Y_{1 j}-\alpha_{1}-\beta_{1} \mu_{x} \\
Y_{2 j}-\alpha_{2}-\beta_{2} \mu_{x} \\
\vdots \\
Y_{p j}-\alpha_{p}-\beta_{p} \mu_{x}
\end{array}\right) \text {, } \\
& =-\frac{n}{2} \frac{1}{\left(\sigma_{x}^{2}\right)}-\frac{1}{2} \sum_{j=1}^{n}-\frac{\left(x_{j}-\mu_{x}\right)^{2}}{\left(\sigma_{x}^{2}\right)^{2}}, \\
& =-\frac{n}{2} \frac{1}{\left(\sigma_{x}^{2}\right)}+\frac{1}{2} \sum_{j=1}^{n} \frac{\left(x_{j}-\mu_{x}\right)^{2}}{\left(\sigma_{x}^{2}\right)^{2}} \text {. }
\end{aligned}
$$

Agora, igualando $\frac{\partial \ln (L(\theta / Z))}{\partial \sigma_{x}^{2}}$ a zero, temos: 


$$
\begin{aligned}
& 0=-\frac{n}{2} \frac{1}{\left(\sigma_{x}^{2}\right)}+\frac{1}{2} \sum_{j=1}^{n} \frac{\left(x_{j}-\mu_{x}\right)^{2}}{\left(\sigma_{x}^{2}\right)^{2}}, \\
& \frac{n}{2} \frac{1}{\left(\sigma_{x}^{2}\right)}=\frac{1}{2} \sum_{j=1}^{n} \frac{\left(x_{j}-\mu_{x}\right)^{2}}{\left(\sigma_{x}^{2}\right)^{2}}, \\
& n=\sum_{j=1}^{n} \frac{\left(x_{j}-\mu_{x}\right)^{2}}{\left(\sigma_{x}^{2}\right)}, \\
& n \sigma_{x}^{2}=\sum_{j=1}^{n}\left(x_{j}-\mu_{x}\right)^{2} .
\end{aligned}
$$

Assim obtemos para o estimador de $\hat{\sigma}_{x}^{2}$ o seguinte resultado:

$\hat{\sigma}_{x}^{2}=\sum_{j=1}^{n} \frac{\left(x_{j}-\hat{\mu}_{x}\right)^{2}}{n}$, que fica:

$$
\hat{\sigma}_{x}^{2}=\sum_{j=1}^{n} \frac{\left(x_{j}-\bar{x}\right)^{2}}{n} .
$$

Agora, passemos a calcular: $\frac{\partial \ln (L(\theta / Z))}{\partial \sigma_{1}^{2}}$, mas antes, temos de calcular a derivada dos seguintes termos de $\ln (L(\theta / Z))$ em relação a $\partial \sigma_{1}^{2}$ :

$$
\begin{aligned}
& {\left[\left(Z-\mu_{z}\right)\right]^{\prime}=\left[\left(\begin{array}{c}
x_{j}-\mu_{x} \\
Y_{1 j}-\alpha_{1}-\beta_{1} \mu_{x} \\
Y_{2 j}-\alpha_{2}-\beta_{2} \mu_{x} \\
\vdots \\
Y_{p j}-\alpha_{p}-\beta_{p} \mu_{x}
\end{array}\right)\right]^{\prime}=\left(\begin{array}{c}
0 \\
0 \\
0 \\
\vdots \\
0
\end{array}\right),} \\
& {\left[-n \ln (2 \pi)-\frac{n}{2} \ln \left(\left|\Sigma_{Z}\right|\right)\right]^{\prime}=\left[-\frac{n}{2} \ln \left(\sigma_{x}^{2} \prod_{i=1}^{p} \sigma_{i}^{2}\right)\right]^{\prime},} \\
& =-\frac{n}{2} \frac{\left(\sigma_{x}^{2} \prod_{i=1}^{p} \sigma_{i}^{2}\right)^{\prime}}{\left(\sigma_{x}^{2} \prod_{i=1}^{p} \sigma_{i}^{2}\right)}=-\frac{n}{2} \frac{\left(\sigma_{x}^{2} \prod_{i=2}^{p} \sigma_{i}^{2}\right)}{\left(\sigma_{x}^{2} \prod_{i=1}^{p} \sigma_{i}^{2}\right)}=-\frac{n}{2} \frac{1}{\left(\left(\sigma_{1}^{2}\right)^{2}\right)}, \\
& \left(\frac{K}{\sigma_{x}^{2}}\right)^{\prime}=\left(\frac{\beta_{i=1}^{2}}{\sigma_{x}^{2}}\right)^{\prime}=-\frac{\beta_{1}^{2}}{\sigma_{1}^{2}}=-\frac{1}{\sigma_{1}^{2}},
\end{aligned}
$$




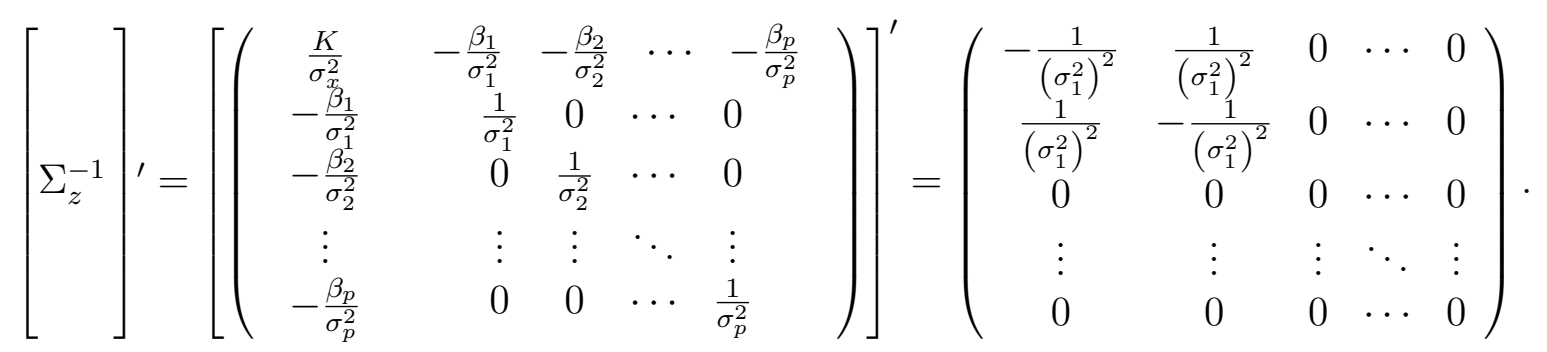

Agora temos:

$$
\begin{aligned}
& \frac{\partial \ln (L(\theta / Z))}{\partial \sigma_{1}^{2}}=\left[-n \ln \left(2 \Pi|\Sigma|^{\frac{1}{2}}\right)-\frac{1}{2} \sum_{j=1}^{n}\left(Z_{j}-\mu_{Z}\right)^{\prime} \Sigma_{Z}^{-1}\left(Z_{j}-\mu_{Z}\right)\right]^{\prime}, \\
& =\left[-n \ln \left(2 \Pi|\Sigma|^{\frac{1}{2}}\right)\right]^{\prime}-\left[\frac{1}{2} \sum_{j=1}^{n}\left(Z_{j}-\mu_{Z}\right)^{\prime} \Sigma_{Z}^{-1}\left(Z_{j}-\mu_{Z}\right)\right]^{\prime}, \\
& =-\frac{n}{2} \frac{1}{\left(\sigma_{1}^{2}\right)}-\frac{1}{2} \sum_{j=1}^{n}\left[\left(Z_{j}-\mu_{Z}\right)^{\prime} \Sigma_{Z}^{-1}\left(Z_{j}-\mu_{Z}\right)\right]^{\prime} \\
& =-\frac{n}{2} \frac{1}{\left(\sigma_{1}^{2}\right)}-\frac{1}{2} \sum_{j=1}^{n}\left[\left(Z_{j}-\mu_{Z}\right)^{t}\right]^{\prime} \Sigma_{Z}^{-1}\left(Z_{j}-\mu_{Z}\right)+\left(Z_{j}-\mu_{Z}\right)^{t}\left[\Sigma_{Z}^{-1}\left(Z_{j}-\mu_{Z}\right)\right]^{\prime}, \\
& =-\frac{n}{2} \frac{1}{\left(\sigma_{1}^{2}\right)}-\frac{1}{2} \sum_{j=1}^{n}\left(Z_{j}-\mu_{Z}\right)^{t}\left[\left(\Sigma_{Z}^{-1}\right)^{\prime}\left(Z_{j}-\mu_{Z}\right)\right. \\
& =-\frac{n}{2} \frac{1}{\left(\sigma_{1}^{2}\right)}-\frac{1}{2} \sum_{j=1}^{n}\left(\begin{array}{c}
x_{j}-\mu_{x} \\
Y_{1 j}-\alpha_{1}-\beta_{1} \mu_{x} \\
Y_{2 j}-\alpha_{2}-\beta_{2} \mu_{x} \\
\vdots \\
Y_{p j}-\alpha_{p}-\beta_{p} \mu_{x}
\end{array}\right)^{t}\left(\begin{array}{ccccc}
-\frac{1}{\left(\sigma_{1}^{2}\right)^{2}} & \frac{1}{\left(\sigma_{1}^{2}\right)^{2}} & 0 & \cdots & 0 \\
\frac{1}{\left(\sigma_{1}^{2}\right)^{2}} & -\frac{1}{\left(\sigma_{1}^{2}\right)^{2}} & 0 & \cdots & 0 \\
0 & 0 & 0 & \cdots & 0 \\
\vdots & \vdots & \vdots & \ddots & \vdots \\
0 & 0 & 0 & \cdots & 0
\end{array}\right)\left(\begin{array}{c}
x_{j}-\mu_{x} \\
Y_{1 j}-\alpha_{1}-\beta_{1} \mu_{x} \\
Y_{2 j}-\alpha_{2}-\beta_{2} \mu_{x} \\
\vdots \\
Y_{p j}-\alpha_{p}-\beta_{p} \mu_{x}
\end{array}\right) \text {, }
\end{aligned}
$$

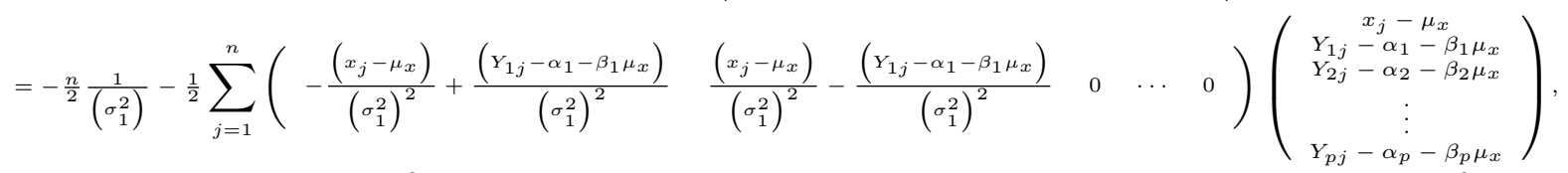

$$
\begin{aligned}
& =-\frac{n}{2} \frac{1}{\left(\sigma_{1}^{2}\right)}-\frac{1}{2} \sum_{j=1}^{n}-\frac{\left(x_{j}-\mu_{x}\right)^{2}}{\left(\sigma_{1}^{2}\right)^{2}}+\frac{\left(x_{j}-\mu_{x}\right)\left(Y_{1 j}-\alpha_{1}-\beta_{1} \mu_{x}\right)}{\left(\sigma_{1}^{2}\right)^{2}}+\frac{\left(x_{j}-\mu_{x}\right)\left(Y_{1 j}-\alpha_{1}-\beta_{1} \mu_{x}\right)}{\left(\sigma_{1}^{2}\right)^{2}}-\frac{\left(Y_{1 j}-\alpha_{1}-\beta_{1} \mu_{x}\right)^{2}}{\left(\sigma_{1}^{2}\right)^{2}}, \\
& =-\frac{n}{2} \frac{1}{\left(\sigma_{1}^{2}\right)}-\frac{1}{2} \sum_{j=1}^{n}-\frac{\left(x_{j}-\mu_{x}\right)^{2}}{\left(\sigma_{1}^{2}\right)^{2}}+2 \frac{\left(x_{j}-\mu_{x}\right)\left(Y_{1 j}-\alpha_{1}-\beta_{1} \mu_{x}\right)}{\left(\sigma_{1}^{2}\right)^{2}}-\frac{\left(Y_{1 j}-\alpha_{1}-\beta_{1} \mu_{x}\right)^{2}}{\left(\sigma_{1}^{2}\right)^{2}} \\
& =-\frac{n}{2} \frac{1}{\left(\sigma_{1}^{2}\right)}+\frac{1}{2} \sum_{j=1}^{n} \frac{\left(x_{j}-\mu_{x}\right)^{2}}{\left(\sigma_{1}^{2}\right)^{2}}-2 \frac{\left(x_{j}-\mu_{x}\right)\left(Y_{1 j}-\alpha_{1}-\beta_{1} \mu_{x}\right)}{\left(\sigma_{1}^{2}\right)^{2}}+\frac{\left(Y_{1 j}-\alpha_{1}-\beta_{1} \mu_{x}\right)^{2}}{\left(\sigma_{1}^{2}\right)^{2}} \text {, }
\end{aligned}
$$

Agora, substituindo $\mu_{x}$ por $\bar{x}, \alpha_{1}$ por 0 e $\beta_{1}$ por 1 temos:

$$
\begin{aligned}
& =-\frac{n}{2} \frac{1}{\left(\sigma_{1}^{2}\right)}+\frac{1}{2} \sum_{j=1}^{n} \frac{\left(x_{j}-\bar{x}\right)^{2}}{\left(\sigma_{1}^{2}\right)^{2}}-2 \frac{\left(x_{j}-\bar{x}\right)\left(Y_{1 j}-0-1 \bar{x}\right)}{\left(\sigma_{1}^{2}\right)^{2}}+\frac{\left(Y_{1 j}-0-1 \bar{x}\right)^{2}}{\left(\sigma_{1}^{2}\right)^{2}}, \\
& =-\frac{n}{2} \frac{1}{\left(\sigma_{1}^{2}\right)}+\frac{1}{2} \sum_{j=1}^{n} \frac{\left(x_{j}-\bar{x}\right)^{2}}{\left(\sigma_{1}^{2}\right)^{2}}-2 \frac{\left(x_{j}-\bar{x}\right)\left(Y_{1 j}-\bar{x}\right)}{\left(\sigma_{1}^{2}\right)^{2}}+\frac{\left(Y_{1 j}-\bar{x}\right)^{2}}{\left(\sigma_{1}^{2}\right)^{2}}
\end{aligned}
$$




$$
\begin{aligned}
& =-\frac{n}{2} \frac{1}{\left(\sigma_{1}^{2}\right)}+\frac{1}{2} \sum_{j=1}^{n} \frac{\left(x_{j}-\bar{x}\right)^{2}}{\left(\sigma_{1}^{2}\right)^{2}}-2 \frac{\left(x_{j}-\bar{x}\right)\left(Y_{1 j}-\bar{x}\right)}{\left(\sigma_{1}^{2}\right)^{2}}+\frac{\left(Y_{1 j}-\bar{x}\right)^{2}}{\left(\sigma_{1}^{2}\right)^{2}}, \\
& =-\frac{n}{2} \frac{1}{\left(\sigma_{1}^{2}\right)}+\frac{1}{2} \sum_{j=1}^{n} \frac{\left(x_{j}-\bar{x}-Y_{1 j}+\bar{x}\right)^{2}}{\left(\sigma_{1}^{2}\right)^{2}}, \\
& =-\frac{n}{2} \frac{1}{\left(\sigma_{1}^{2}\right)}+\frac{1}{2} \sum_{j=1}^{n} \frac{\left(x_{j}-Y_{1 j}\right)^{2}}{\left(\sigma_{1}^{2}\right)^{2}}, \\
& =-\frac{n}{2} \frac{1}{\left(\sigma_{1}^{2}\right)}+\frac{1}{2\left(\sigma_{1}^{2}\right)^{2}} \sum_{j=1}^{n}\left(x_{j}-Y_{1 j}\right)^{2} . \\
& \text { Igualando } \frac{\partial \ln (L(\theta / Z))}{\partial \sigma_{1}^{2}} \text { a zero, temos: }
\end{aligned}
$$

$\frac{n}{2} \frac{1}{\left(\sigma_{1}^{2}\right)}=\frac{1}{2\left(\sigma_{1}^{2}\right)^{2}} \sum_{j=1}^{n}\left(x_{j}-Y_{1 j}\right)^{2}$

ou seja,

$$
\left(\hat{\sigma}_{1}^{2}\right)=\frac{\sum_{j=1}^{n}\left(x_{j}-Y_{1 j}\right)^{2}}{n} .
$$

Agora, passemos a calcular: $\frac{\partial \ln (L(\theta / Z))}{\partial \sigma_{i}^{2}}$, considerando $i \geq 1$, mas antes, temos de calcular a derivada dos seguintes termos de $\ln (L(\theta / Z))$ em relação a $\partial \sigma_{i}^{2}$ :

$$
\begin{aligned}
& {\left[\left(Z-\mu_{z}\right)\right]^{\prime}=\left[\left(\begin{array}{c}
x_{j}-\mu_{x} \\
Y_{1 j}-\alpha_{1}-\beta_{1} \mu_{x} \\
Y_{2 j}-\alpha_{2}-\beta_{2} \mu_{x} \\
\vdots \\
Y_{p j}-\alpha_{p}-\beta_{p} \mu_{x}
\end{array}\right)\right]^{\prime}=\left(\begin{array}{c}
0 \\
0 \\
0 \\
\vdots \\
0
\end{array}\right),} \\
& {\left[-n \ln (2 \pi)-\frac{n}{2} \ln \left(\left|\Sigma_{Z}\right|\right)\right]^{\prime}=\left[-\frac{n}{2} \ln \left(\sigma_{x}^{2} \prod_{i=1}^{p} \sigma_{i}^{2}\right)\right]^{\prime},} \\
& =-\frac{n}{2} \frac{\left(\sigma_{x}^{2} \prod_{i=1}^{p} \sigma_{i}^{2}\right)^{\prime}}{\left(\sigma_{x}^{2} \prod_{i=1}^{p} \sigma_{i}^{2}\right)}=-\frac{n}{2} \frac{1}{\left(\sigma_{2}^{2}\right)^{2}}, i \neq 2 \\
& \left(\frac{K}{\sigma_{x}^{2}}\right)^{\prime}=\left(\frac{p}{1+\sigma_{x}^{2} \sum_{i=1}^{p} \frac{\beta_{i}^{2}}{\sigma_{i}^{2}}}\right)^{\sigma_{x}^{2}}=-\frac{\beta_{2}^{2}}{\left(\sigma_{2}^{2}\right)^{2}},
\end{aligned}
$$




$$
\left[\Sigma_{z}^{-1}\right]^{\prime}=\left[\left(\begin{array}{ccccc}
\frac{k}{\sigma_{x}^{2}} & -\frac{\beta_{1}}{\sigma_{1}^{2}} & -\frac{\beta_{2}}{\sigma_{2}^{2}} & \cdots & -\frac{\beta_{p}}{\sigma_{p}^{2}} \\
-\frac{\beta_{1}}{\sigma_{1}^{2}} & \frac{1}{\sigma_{1}^{2}} & 0 & \cdots & 0 \\
-\frac{\beta_{2}}{\sigma_{2}^{2}} & 0 & \frac{1}{\sigma_{2}^{2}} & \cdots & 0 \\
\vdots & \vdots & \vdots & \ddots & \vdots \\
-\frac{\beta_{p}}{\sigma_{p}^{2}} & 0 & 0 & \cdots & \frac{1}{\sigma_{p}^{2}}
\end{array}\right)\right]^{\prime}=\left(\begin{array}{ccccc}
-\frac{\beta_{2}^{2}}{\left(\sigma_{2}^{2}\right)^{2}} & 0 & \frac{\beta_{2}}{\left(\sigma_{2}^{2}\right)^{2}} & \cdots & 0 \\
0 & 0 & 0 & \cdots & 0 \\
\frac{\beta_{2}}{\left(\sigma_{2}^{2}\right)^{2}} & 0 & -\frac{1}{\left(\sigma_{2}^{2}\right)^{2}} & \cdots & 0 \\
\vdots & \vdots & \vdots & \ddots & \vdots \\
0 & 0 & 0 & \cdots & 0
\end{array}\right) .
$$

Agora, temos:

$$
\begin{aligned}
& \frac{\partial \ln (L(\theta / Z))}{\partial \sigma_{i}^{2}}=\left[-n \ln \left(2 \Pi|\Sigma|^{\frac{1}{2}}\right)-\frac{1}{2} \sum_{j=1}^{n}\left(Z_{j}-\mu_{Z}\right)^{\prime} \Sigma_{Z}^{-1}\left(Z_{j}-\mu_{Z}\right)\right]^{\prime}, \\
& =\left[-n \ln \left(2 \Pi|\Sigma|^{\frac{1}{2}}\right)\right]^{\prime}-\left[\frac{1}{2} \sum_{j=1}^{n}\left(Z_{j}-\mu_{Z}\right)^{\prime} \Sigma_{Z}^{-1}\left(Z_{j}-\mu_{Z}\right)\right]^{\prime}, \\
& =-\frac{n}{2} \frac{1}{\left(\sigma_{2}^{2}\right)}-\frac{1}{2} \sum_{j=1}^{n}\left[\left(Z_{j}-\mu_{Z}\right)^{\prime} \Sigma_{Z}^{-1}\left(Z_{j}-\mu_{Z}\right)\right]^{\prime} \\
& =-\frac{n}{2} \frac{1}{\left(\sigma_{2}^{2}\right)}-\frac{1}{2} \sum_{j=1}^{n}\left[\left(Z_{j}-\mu_{Z}\right)^{t}\right]^{\prime} \Sigma_{Z}^{-1}\left(Z_{j}-\mu_{Z}\right)+\left(Z_{j}-\mu_{Z}\right)^{t}\left[\Sigma_{Z}^{-1}\left(Z_{j}-\mu_{Z}\right)\right]^{\prime} \text {, } \\
& =-\frac{n}{2} \frac{1}{\left(\sigma_{2}^{2}\right)}-\frac{1}{2} \sum_{j=1}^{n}\left(Z_{j}-\mu_{Z}\right)^{t}\left(\Sigma_{Z}^{-1}\right)^{\prime}\left(Z_{j}-\mu_{Z}\right) \\
& =-\frac{n}{2} \frac{1}{\left(\sigma_{2}^{2}\right)}-\frac{1}{2} \sum_{j=1}^{n}\left(\begin{array}{c}
x_{j}-\mu_{x} \\
Y_{1 j}-\alpha_{1}-\beta_{1} \mu_{x} \\
Y_{2 j}-\alpha_{2}-\beta_{2} \mu_{x} \\
\vdots \\
Y_{p j}-\alpha_{p}-\beta_{p} \mu_{x}
\end{array}\right)^{t}\left(\begin{array}{ccccc}
-\frac{\beta_{2}^{2}}{\left(\sigma_{2}^{2}\right)^{2}} & 0 & \frac{\beta_{2}}{\left(\sigma_{2}^{2}\right)^{2}} & \cdots & 0 \\
0 & 0 & 0 & \cdots & 0 \\
\frac{\beta_{2}}{\left(\sigma_{2}^{2}\right)^{2}} & 0 & -\frac{1}{\left(\sigma_{2}^{2}\right)^{2}} & \cdots & 0 \\
\vdots & \vdots & \vdots & \ddots & \vdots \\
0 & 0 & 0 & \cdots & 0
\end{array}\right)\left(\begin{array}{c}
x_{j}-\mu_{x} \\
Y_{1 j}-\alpha_{1}-\beta_{1} \mu_{x} \\
Y_{2 j}-\alpha_{2}-\beta_{2} \mu_{x} \\
\vdots \\
Y_{p j}-\alpha_{p}-\beta_{p} \mu_{x}
\end{array}\right) \text {, } \\
& =-\frac{n}{2} \frac{1}{\left(\sigma_{2}^{2}\right)}-\frac{1}{2} \sum_{j=1}^{n}\left(\begin{array}{llll}
-\frac{\beta_{2}^{2}\left(x_{j}-\mu_{x}\right)}{\left(\sigma_{2}^{2}\right)^{2}}+\frac{\beta_{2}\left(y_{2 j}-\alpha_{2}-\beta_{2} \mu_{x}\right)}{\left(\sigma_{2}^{2}\right)^{2}} \quad 0 \quad \frac{\beta_{2}\left(x_{j}-\mu_{x}\right)}{\left(\sigma_{2}^{2}\right)^{2}}-\frac{\left(y_{2 j}-\alpha_{2}-\beta_{2} \mu_{x}\right)}{\left(\sigma_{2}^{2}\right)^{2}} \cdots \cdots & \cdots
\end{array}\right)\left(\begin{array}{c}
x_{j}-\mu_{x} \\
Y_{2 j}-\alpha_{1}-\beta_{1} \mu_{x} \\
\vdots \\
\vdots \\
Y_{p j}-\beta_{2} \mu_{x}-\beta_{p} \mu_{x}
\end{array}\right) \text {, } \\
& =-\frac{n}{2} \frac{1}{\left(\sigma_{2}^{2}\right)}-\frac{1}{2} \sum_{j=1}^{n}-\frac{\beta_{2}^{2}\left(x_{j}-\mu_{x}\right)^{2}}{\left(\sigma_{2}^{2}\right)^{2}}+\frac{\beta_{2}\left(x_{j}-\mu_{x}\right)\left(Y_{2 j}-\alpha_{2}-\beta_{2} \mu_{x}\right)}{\left(\sigma_{2}^{2}\right)^{2}}+\frac{\beta_{2}\left(x_{j}-\mu_{x}\right)\left(Y_{2 j}-\alpha_{2}-\beta_{2} \mu_{x}\right)}{\left(\sigma_{2}^{2}\right)^{2}}-\frac{\left(Y_{2 j}-\alpha_{2}-\beta_{2} \mu_{x}\right)^{2}}{\left(\sigma_{2}^{2}\right)^{2}} \text {, } \\
& =-\frac{n}{2} \frac{1}{\left(\sigma_{2}^{2}\right)}-\frac{1}{2} \sum_{j=1}^{n}-\frac{\beta_{2}^{2}\left(x_{j}-\mu_{x}\right)^{2}}{\left(\sigma_{2}^{2}\right)^{2}}+2 \frac{\beta_{2}\left(x_{j}-\mu_{x}\right)\left(Y_{2 j}-\alpha_{2}-\beta_{2} \mu_{x}\right)}{\left(\sigma_{2}^{2}\right)^{2}}-\frac{\left(Y_{2 j}-\alpha_{2}-\beta_{2} \mu_{x}\right)^{2}}{\left(\sigma_{2}^{2}\right)^{2}}, \\
& =-\frac{n}{2} \frac{1}{\left(\sigma_{2}^{2}\right)}+\frac{1}{2} \sum_{j=1}^{n} \frac{\beta_{2}^{2}\left(x_{j}-\mu_{x}\right)^{2}}{\left(\sigma_{2}^{2}\right)^{2}}-2 \frac{\beta_{2}\left(x_{j}-\mu_{x}\right)\left(Y_{2 j}-\alpha_{2}-\beta_{2} \mu_{x}\right)}{\left(\sigma_{2}^{2}\right)^{2}}+\frac{\left(Y_{2 j}-\alpha_{2}-\beta_{2} \mu_{x}\right)^{2}}{\left(\sigma_{2}^{2}\right)^{2}} \text {, } \\
& =-\frac{n}{2} \frac{1}{\left(\sigma_{2}^{2}\right)}+\frac{1}{2\left(\sigma_{2}^{2}\right)^{2}} \sum_{j=1}^{n} \beta_{2}^{2}\left(x_{j}-\mu_{x}\right)^{2}-2 \beta_{2}\left(x_{j}-\mu_{x}\right)\left(Y_{2 j}-\alpha_{2}-\beta_{2} \mu_{x}\right)+\left(Y_{2 j}-\alpha_{2}-\beta_{2} \mu_{x}\right)^{2} \text {, } \\
& =-\frac{n}{2} \frac{1}{\left(\sigma_{2}^{2}\right)}+\frac{1}{2\left(\sigma_{2}^{2}\right)^{2}} \sum_{j=1}^{n}\left[\beta_{2}\left(x_{j}-\mu_{x}\right)-\left(Y_{2 j}-\alpha_{2}-\beta_{2} \mu_{x}\right)\right]^{2} \text {. }
\end{aligned}
$$

Agora, substituindo $\alpha_{2}$ por $\bar{Y}_{2}-\beta_{2} \mu_{x}$ temos: 


$$
\begin{aligned}
& =-\frac{n}{2} \frac{1}{\left(\sigma_{2}^{2}\right)}+\frac{1}{2\left(\sigma_{2}^{2}\right)^{2}} \sum_{j=1}^{n}\left[\beta_{2}\left(x_{j}-\mu_{x}\right)-\left(Y_{2 j}-\left(\bar{Y}_{2}-\beta_{2} \mu_{x}\right)-\beta_{2} \mu_{x}\right)\right]^{2}, \\
& =-\frac{n}{2} \frac{1}{\left(\sigma_{2}^{2}\right)}+\frac{1}{2\left(\sigma_{2}^{2}\right)^{2}} \sum_{j=1}^{n}\left[\beta_{2}\left(x_{j}-\mu_{x}\right)-\left(Y_{2 j}-\bar{Y}_{2}+\beta_{2} \mu_{x}-\beta_{2} \mu_{x}\right)\right]^{2} \\
& =-\frac{n}{2} \frac{1}{\left(\sigma_{2}^{2}\right)}+\frac{1}{2\left(\sigma_{2}^{2}\right)^{2}} \sum_{j=1}^{n}\left[\beta_{2}\left(x_{j}-\mu_{x}\right)-\left(Y_{2 j}-\bar{Y}_{2}\right)\right]^{2} .
\end{aligned}
$$

Agora, substituindo $\mu_{x}$ por $\bar{x}$ temos:

$=-\frac{n}{2} \frac{1}{\left(\sigma_{2}^{2}\right)}+\frac{1}{2\left(\sigma_{2}^{2}\right)^{2}} \sum_{j=1}^{n}\left[\beta_{2}\left(x_{j}-\bar{x}\right)-\left(Y_{2 j}-\bar{Y}_{2}\right)\right]^{2}$.

Igualando $\frac{\partial \ln (L(\theta / Z))}{\partial \sigma_{i}^{2}}$ a zero, temos:

$\frac{n}{2} \frac{1}{\left(\sigma_{2}^{2}\right)}=\frac{1}{2\left(\sigma_{2}^{2}\right)^{2}} \sum_{j=1}^{n}\left[\beta_{2}\left(x_{j}-\bar{x}\right)-\left(Y_{2 j}-\bar{Y}_{2}\right)\right]^{2}$,

$n=\frac{1}{\left(\sigma_{2}^{2}\right)} \sum_{j=1}^{n}\left[\beta_{2}\left(x_{j}-\bar{x}\right)-\left(Y_{2 j}-\bar{Y}_{2}\right)\right]^{2}$,

$n\left(\sigma_{2}^{2}\right)=\sum_{j=1}^{n}\left[\beta_{2}\left(x_{j}-\bar{x}\right)-\left(Y_{2 j}-\bar{Y}_{2}\right)\right]^{2}$,

que resulta em:

$\hat{\sigma}_{2}^{2}=\frac{1}{n} \sum_{j=1}^{n}\left[\beta_{2}\left(x_{j}-\bar{x}\right)-\left(Y_{2 j}-\bar{Y}_{2}\right)\right]^{2}$,

que generalizando temos:

$$
\hat{\sigma}_{i}^{2}=\frac{1}{n} \sum_{j=1}^{n}\left[\beta_{i}\left(x_{j}-\bar{x}\right)-\left(Y_{i j}-\bar{Y}_{i}\right)\right]^{2}
$$

resultado válido para $i \geq 2$. 


\section{Apêndice E}

\section{Cálculos dos valores para esperança e variância para o Modelo Completo}

Neste caso, temos:

$$
f(\theta / Z)=\frac{1}{(2 \pi)\left|\sum_{z}\right|^{\frac{1}{2}}} \exp -\frac{1}{2}\left(Z-\mu_{z}\right)^{t} \Sigma_{z}^{-1}\left(Z-\mu_{z}\right)
$$

onde:

$$
\begin{gathered}
\theta / Z \sim N\left(\mu_{z}, \Sigma_{z}\right), \\
Z=\left(\begin{array}{c}
x_{j} \\
Y_{1 j} \\
Y_{2 j} \\
\vdots \\
Y_{p j}
\end{array}\right), \mu_{Z}=\left(\begin{array}{c}
\mu_{x} \\
\alpha_{1}+\beta_{1} \mu_{x} \\
\alpha_{2}+\beta_{2} \mu_{x} \\
\vdots \\
\alpha_{p}+\beta_{p} \mu_{p}
\end{array}\right) e \Sigma_{z}=\sigma_{x}^{2}\left(\begin{array}{cc}
1 & \beta^{\prime} \\
\beta & \beta \beta^{\prime}+A
\end{array}\right),
\end{gathered}
$$

com:

$$
A=\operatorname{diag}\left(\frac{\sigma_{1}^{2}}{\sigma_{x}^{2}}, \cdots, \frac{\sigma_{p}^{2}}{\sigma_{x}^{2}}\right) .
$$

Agora, queremos calcular:

$$
\hat{x}_{j}=\mathbb{E}\left[x_{j} / Y_{j} ; \theta\right]=\mu_{x}+\Sigma_{12} \Sigma_{22}^{-1}\left(Y-\mu_{Y}\right),
$$

Conforme está descrito em Johnson et al. (1998). 
onde:

$$
X=x, Y_{j}=\left(\begin{array}{c}
Y_{1 j} \\
Y_{2 j} \\
\vdots \\
Y_{p j}
\end{array}\right)
$$

e $\Sigma_{11}=\sigma_{x}^{2}, \Sigma_{12}=\beta^{\prime} \sigma_{x}^{2}, \Sigma_{21}=\beta \sigma_{x}^{2}, \Sigma_{22}=\sigma_{x}^{2}\left(\beta \beta^{\prime}+A\right)$.

Agora, passemos a calcular $\Sigma_{22}^{-1}$, que pode ser escrito como:

$$
\Sigma_{22}^{-1}=\frac{1}{\sigma_{x}^{2}}\left[A^{-1}-\frac{1 \cdot A^{-1} \beta \beta^{\prime} A^{-1}}{1+\beta^{\prime} A^{-1} \beta}\right] \text {, com: }
$$$$
A^{-1}=\left(\begin{array}{cccc}
\frac{\sigma_{x}^{2}}{\sigma_{1}^{2}} & 0 & \cdots & 0 \\
0 & \frac{\sigma_{x}^{2}}{\sigma_{2}^{2}} & \cdots & 0 \\
\vdots & \vdots & \ddots & \vdots \\
0 & 0 & \cdots & \frac{\sigma_{x}^{2}}{\sigma_{p}^{2}}
\end{array}\right) \text {. Então: }
$$

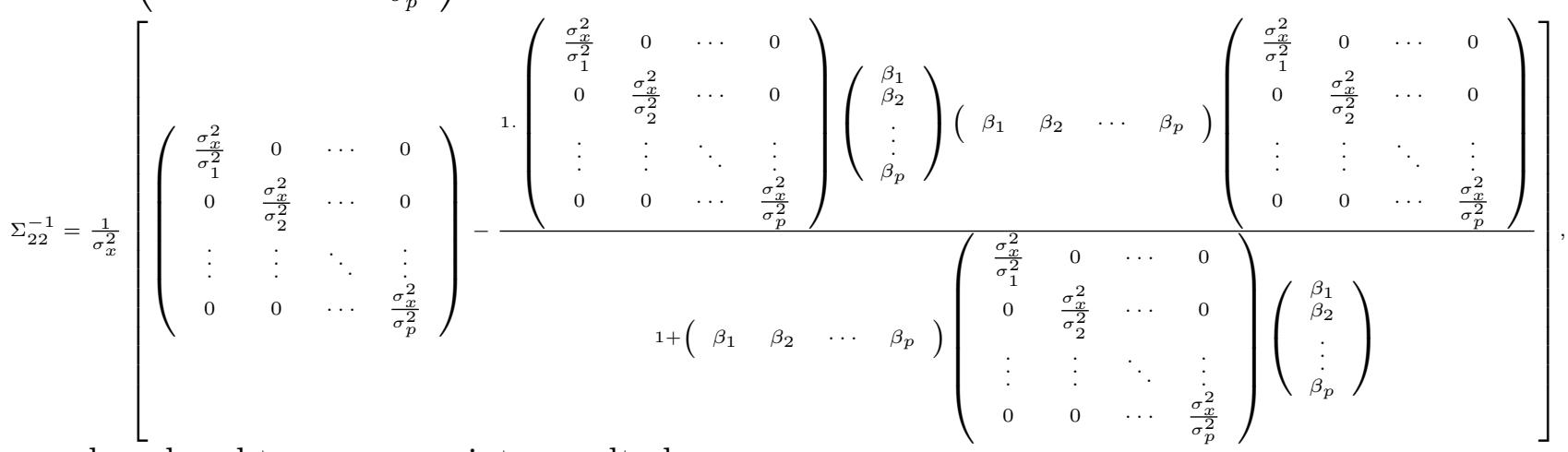

que resolvendo, obtemos o seguinte resultado:

$$
\Sigma_{22}^{-1}=\left[\left(\begin{array}{cccc}
\frac{1}{\sigma_{1}^{2}} & 0 & \cdots & 0 \\
0 & \frac{1}{\sigma_{2}^{2}} & \cdots & 0 \\
\vdots & \vdots & \ddots & \vdots \\
0 & 0 & \cdots & \frac{1}{\sigma_{p}^{2}}
\end{array}\right)-\frac{\sigma_{x}^{2}}{K}\left(\begin{array}{cccc}
\frac{\beta_{1}^{2}}{\left(\sigma_{1}^{2}\right)^{2}} & \frac{\beta_{1} \beta_{2}}{\sigma_{1}^{2} \sigma_{2}^{2}} & \cdots & \frac{\beta_{1} \beta_{p}}{\sigma_{1}^{2} \sigma_{p}^{2}} \\
\frac{\beta_{1} \beta_{2}}{\sigma_{1}^{2} \sigma_{2}^{2}} & \frac{\beta_{2}^{2}}{\left(\sigma_{2}^{2}\right)^{2}} & \cdots & \frac{\beta_{2} \beta_{p}}{\sigma_{2}^{2} \sigma_{p}^{2}} \\
\vdots & \vdots & \ddots & \vdots \\
\frac{\beta_{1} \beta_{p}}{\sigma_{1}^{2} \sigma_{p}^{2}} & \frac{\beta_{2} \beta_{p}}{\sigma_{2}^{2} \sigma_{p}^{2}} & \cdots & \frac{\beta_{p}^{2}}{\left(\sigma_{p}^{2}\right)^{2}}
\end{array}\right)\right] .
$$

Agora, para $\hat{x}_{j}$ temos:

$$
\hat{x}_{j}=\mu_{x}+\sigma_{x}^{2}\left(\beta_{1}, \beta_{2}, \cdots, \beta_{p}\right)
$$




$$
\begin{aligned}
& {\left[\left(\begin{array}{cccc}
\frac{1}{\sigma_{1}^{2}} & 0 & \cdots & 0 \\
0 & \frac{1}{\sigma_{2}^{2}} & \cdots & 0 \\
\vdots & \vdots & \ddots & \vdots \\
0 & 0 & \cdots & \frac{1}{\sigma_{p}^{2}}
\end{array}\right)-\frac{\sigma_{x}^{2}}{K}\left(\begin{array}{cccc}
\frac{\beta_{1}^{2}}{\left(\sigma_{1}^{2}\right)^{2}} & \frac{\beta_{1} \beta_{2}}{\sigma_{1}^{2} \sigma_{2}^{2}} & \cdots & \frac{\beta_{1} \beta_{p}}{\sigma_{1}^{2} \sigma_{p}^{2}} \\
\frac{\beta_{1} \beta_{2}}{\sigma_{1}^{2} \sigma_{2}^{2}} & \frac{\beta_{2}^{2}}{\left(\sigma_{2}^{2}\right)^{2}} & \cdots & \frac{\beta_{2} \beta_{p}}{\sigma_{2}^{2} \sigma_{p}^{2}} \\
\vdots & \vdots & \ddots & \vdots \\
\frac{\beta_{1} \beta_{p}}{\sigma_{1}^{2} \sigma_{p}^{2}} & \frac{\beta_{2} \beta_{p}}{\sigma_{2}^{2} \sigma_{p}^{2}} & \cdots & \frac{\beta_{p}^{2}}{\left(\sigma_{p}^{2}\right)^{2}}
\end{array}\right)\right]} \\
& \left(\begin{array}{c}
Y_{1 j}-\alpha_{1}-\beta_{1} \mu_{x} \\
Y_{2 j}-\alpha_{2}-\beta_{2} \mu_{x} \\
\vdots \\
Y_{p j}-\alpha_{p}-\beta_{p} \mu_{x}
\end{array}\right), \\
& \hat{x}_{j}=\mu_{x}+\sigma_{x}^{2} \\
& \left.=\left[\begin{array}{llll}
\beta_{1} & \beta_{2} & \cdots & \beta_{p}
\end{array}\right)\left(\begin{array}{cccc}
\frac{1}{\sigma_{1}^{2}} & 0 & \cdots & 0 \\
0 & \frac{1}{\sigma_{2}^{2}} & \cdots & 0 \\
\vdots & \vdots & \ddots & \vdots \\
0 & 0 & \cdots & \frac{1}{\sigma_{p}^{2}}
\end{array}\right)-\frac{\sigma_{x}^{2}}{K}\left(\begin{array}{llll}
\beta_{1} & \beta_{2} & \cdots & \beta_{p}
\end{array}\right)\left(\begin{array}{cccc}
\frac{\beta_{1}^{2}}{\left(\sigma_{1}^{2}\right)^{2}} & \frac{\beta_{1} \beta_{2}}{\sigma_{1}^{2} \sigma_{2}^{2}} & \cdots & \frac{\beta_{1} \beta_{p}}{\sigma_{1}^{2} \sigma_{p}^{2}} \\
\frac{\beta_{1} \beta_{2}}{\sigma_{1}^{2} \sigma_{2}^{2}} & \frac{\beta_{2}^{2}}{\left(\sigma_{2}^{2}\right)^{2}} & \cdots & \frac{\beta_{2} \beta_{p}}{\sigma_{2}^{2} \sigma_{p}^{2}} \\
\vdots & \vdots & \ddots & \vdots \\
\frac{\beta_{1} \beta_{p}}{\sigma_{1}^{2} \sigma_{p}^{2}} & \frac{\beta_{2} \beta_{p}}{\sigma_{2}^{2} \sigma_{p}^{2}} & \cdots & \frac{\beta_{p}^{2}}{\left(\sigma_{p}^{2}\right)^{2}}
\end{array}\right)\right] \\
& \left(\begin{array}{c}
Y_{1 j}-\alpha_{1}-\beta_{1} \mu_{x} \\
Y_{2 j}-\alpha_{2}-\beta_{2} \mu_{x} \\
\vdots \\
Y_{p j}-\alpha_{p}-\beta_{p} \mu_{x}
\end{array}\right),
\end{aligned}
$$

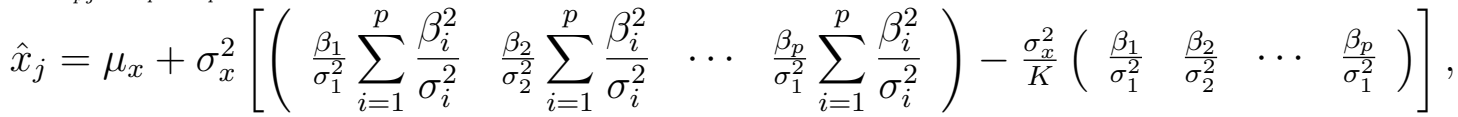

$$
\begin{aligned}
& \left(\begin{array}{c}
Y_{1 j}-\alpha_{1}-\beta_{1} \mu_{x} \\
Y_{2 j}-\alpha_{2}-\beta_{2} \mu_{x} \\
\vdots \\
Y_{p j}-\alpha_{p}-\beta_{p} \mu_{x}
\end{array}\right)
\end{aligned}
$$

ou seja,

$$
\hat{x}_{j}=\mu_{x}+\frac{1}{K}\left[\frac{\sigma_{x}^{2} Y_{1 j}}{\sigma_{1}^{2}}-\mu_{x}(K-1)+\beta^{*} A^{-2}\left(Y_{i j}-\alpha_{i}\right)\right] .
$$

Agora, temos de calcular $\operatorname{Var}\left(x_{j} / Y_{j}, \theta\right)$, que pode ser obtida através da seguinte fórmula:

$$
\operatorname{Var}\left(x_{j} / Y_{j}, \theta\right)=\Sigma_{11}-\Sigma_{12} \Sigma_{22}^{-1} \Sigma_{21}
$$

Então, 
$\operatorname{Var}\left(x_{j} / Y_{j}, \theta\right)=1 . \sigma_{x}^{2}-\beta^{\prime} \sigma_{x}^{2}\left[\left(\begin{array}{cccc}\frac{1}{\sigma_{1}^{2}} & 0 & \cdots & 0 \\ 0 & \frac{1}{\sigma_{2}^{2}} & \cdots & 0 \\ \vdots & \vdots & \ddots & \vdots \\ 0 & 0 & \cdots & \frac{1}{\sigma_{p}^{2}}\end{array}\right)-\frac{\sigma_{x}^{2}}{K}\left(\begin{array}{cccc}\frac{\beta_{1}^{2}}{\left(\sigma_{1}^{2}\right)^{2}} & \frac{\beta_{1} \beta_{2}}{\sigma_{1}^{2} \sigma_{2}^{2}} & \cdots & \frac{\beta_{1} \beta_{p}}{\sigma_{1}^{2} \sigma_{p}^{2}} \\ \frac{\beta_{1} \beta_{2}}{\sigma_{1}^{2} \sigma_{2}^{2}} & \frac{\beta_{2}^{2}}{\left(\sigma_{2}^{2}\right)^{2}} & \cdots & \frac{\beta_{2} \beta_{p}}{\sigma_{2}^{2} \sigma_{p}^{2}} \\ \vdots & \vdots & \ddots & \vdots \\ \frac{\beta_{1} \beta_{p}}{\sigma_{1}^{2} \sigma_{p}^{2}} & \frac{\beta_{2} \beta_{p}}{\sigma_{2}^{2} \sigma_{p}^{2}} & \cdots & \frac{\beta_{p}^{2}}{\left(\sigma_{p}^{2}\right)^{2}}\end{array}\right)\right] \beta \sigma_{x}^{2}$, que resolvendo, obtemos o seguinte resultado:

$\operatorname{Var}\left(x_{j} / Y_{j}, \theta\right)=\frac{\sigma_{x}^{2}}{K}$,

Portanto:

$$
\operatorname{Var}\left(x_{j} / Y_{j}, \theta\right)=\frac{\sigma_{x}^{2}}{K} .
$$

Assim, teremos:

$$
\mathbb{E}\left[x_{j}^{2} / Y_{j}, \theta\right]=\hat{x}_{j}^{2}+\frac{\sigma_{x}^{2}}{K}
$$




\section{Apêndice F}

\section{Matriz de informação esperada.}

Nesta seção consideramos a matriz de informação de Fisher do modelo ob-

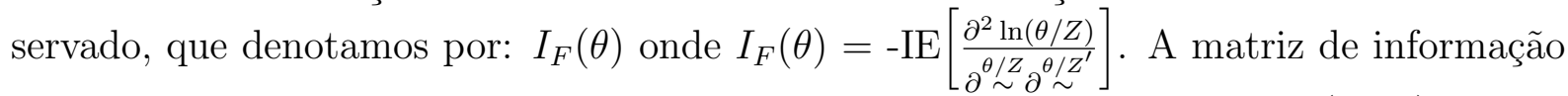
para o modelo de calibração comparativa calculada em Bolfarine e Rojas (1995). Segue das propriedades assintóticas dos estimadores de máxima verossimilhança para $n$ grande,

$$
\hat{\theta} \approx N\left(\theta, I_{F}^{-1}(\theta)\right) .
$$

Os elementos da matriz de informação esperada $I_{F}^{-1}(\theta)$ derivada em Rojas e Bolfarine (1995) são dados por:

$$
\begin{gathered}
I_{\mu_{x} \mu_{x}}=\frac{n\left(1-\frac{1}{\sigma_{x}^{2} v}\right)}{\sigma_{x}^{2}} ; \\
I_{\mu_{x} \alpha_{k}}=\frac{n \beta_{k}}{v \sigma_{k}^{2} \sigma_{x}^{2}} ; \\
I_{\mu_{x} \beta_{k}}=\frac{n \beta_{k} \mu_{x}}{v \sigma_{x}^{2} \sigma_{k}^{2}} ; \\
I_{\mu_{x} \sigma_{x}^{2}}=I_{\mu_{x} \sigma_{1}^{2}}=I_{\mu_{x} \sigma_{k}^{2}}=0 ; \\
I_{\alpha_{k} \alpha_{k}}=\frac{n}{\sigma_{k}^{2}}\left(1-\frac{\beta_{k}^{2}}{v \sigma_{k}^{2}}\right) ; \\
I_{\alpha_{k} \alpha_{l}}=-\frac{n \beta_{k} \beta_{l}}{v \sigma_{k}^{2} \sigma_{l}^{2}} ;
\end{gathered}
$$




$$
\begin{aligned}
& I_{\alpha_{k} \beta_{k}}=-\frac{n \beta_{k}^{2} \mu_{x}}{v \sigma_{k}^{4}}+\frac{n \mu_{x}}{\sigma_{k}^{2}} \\
& I_{\alpha_{k} \beta_{l}}=-\frac{n \beta_{k} \beta_{l} \mu_{x}}{v \sigma_{k}^{2} \sigma_{l}^{2}} \\
& I_{\alpha_{x} \sigma_{x}^{2}}=I_{\alpha_{k} \sigma_{1}^{2}}=I_{\alpha_{k} \sigma_{k}^{2}}=I_{\alpha_{k} \sigma_{l}^{2}}=0 ; \\
& I_{\beta_{k} \beta_{k}}=\frac{n}{\sigma_{k}^{2}}\left(\mu_{x}^{2}+\sigma_{x}^{2}-1 v\right)+\frac{n \beta_{k}^{2}}{v \sigma_{k}^{4}}\left(2 / v-\sigma_{x}^{2}-\mu_{x}^{2}\right) ; \\
& I_{\beta_{k} \beta_{l}}=\frac{n \beta_{k} \beta_{l}}{v \sigma_{k}^{2} \sigma_{l}^{2}}\left(2 v-\left(\sigma_{x}^{2}+\mu_{x}^{2}\right)\right) \\
& I_{\beta_{k} \sigma_{x}^{2}}=\frac{n \beta_{k}}{v \sigma_{k}^{2} \sigma_{x}^{4}}\left(\sigma_{x}^{2}-1 / v\right) \\
& I_{\beta_{k} \sigma_{1}^{2}}=-\frac{n \beta_{k}}{v^{2} \sigma_{k}^{2} \sigma_{1}^{4}} \\
& I_{\beta_{k} \sigma_{k}^{2}}=\frac{n \beta_{k}}{v \sigma_{k}^{4}}-\frac{n \beta_{k}^{3}}{v^{2} \sigma_{k}^{6}} ; \\
& I_{\beta_{k} \sigma_{l}^{2}}=-\frac{n \beta_{k} \beta_{l}^{2}}{v^{2} \sigma_{k}^{2} \sigma_{l}^{4}} \\
& I_{\sigma_{x}^{2} \sigma_{x}^{2}}=\frac{n}{2 \sigma_{x}^{4}}-\frac{n}{v \sigma_{x}^{6}}+\frac{n}{2 v^{2} \sigma_{x}^{8}} \\
& I_{\sigma_{x}^{2} \sigma_{l}^{2}}=\frac{n}{2 v^{2} \sigma_{x}^{4} \sigma_{1}^{4}} \\
& I_{\sigma_{x}^{2} \sigma_{k}^{2}}=\frac{n \beta_{k}^{2}}{2 v^{2} \sigma_{x}^{4} \sigma_{1}^{4}}
\end{aligned}
$$




$$
\begin{gathered}
I_{\sigma_{l}^{2} \sigma_{l}^{2}}=\frac{n}{2 \sigma_{l}^{4}}-\frac{n}{v \sigma_{l}^{6}}+\frac{n}{2 v^{2} \sigma_{l}^{8}} ; \\
I_{\sigma_{1}^{2} \sigma_{k}^{2}}=\frac{n \beta_{k}^{2}}{2 v^{2} \sigma_{1}^{4} \sigma_{k}^{4}} ; \\
I_{\sigma_{k}^{2} \sigma_{k}^{2}}=\frac{n}{2 \sigma_{k}^{4}}-\frac{n \beta_{k}^{2}}{v \sigma_{k}^{6}}+\frac{n \beta_{k}^{4}}{2 v^{2} \sigma_{k}^{8}} ; \\
I_{\sigma_{k}^{2} \sigma_{l}^{2}}=\frac{n \beta_{k}^{2} \beta_{l}^{2}}{2 v^{2} \sigma_{k}^{4} \sigma_{l}^{4}} ; \\
\operatorname{com} v=\frac{1}{\sigma_{x}^{2}}+\sum_{i=1}^{p} \frac{\beta_{i}^{2}}{\sigma_{i}^{2}} ; k \neq l=2, \ldots, p .
\end{gathered}
$$




\section{Apêndice G}

\section{Programas utilizados.}

Para a elaboração dessa dissertação foram desenvolvidas ou utilizadas adaptações de diversos programas feitos, utilizando o software Ox. $=3$ instrumentos.

O primeiro programa foi desenvolvido para fazer simulações, considerando $\mathrm{p}$

O segundo programa foi desenvolvido também em Ox para que seja utilizado os dados reais das árvores também para $\mathrm{p}=3$ instrumentos.

Primeiro Programa.

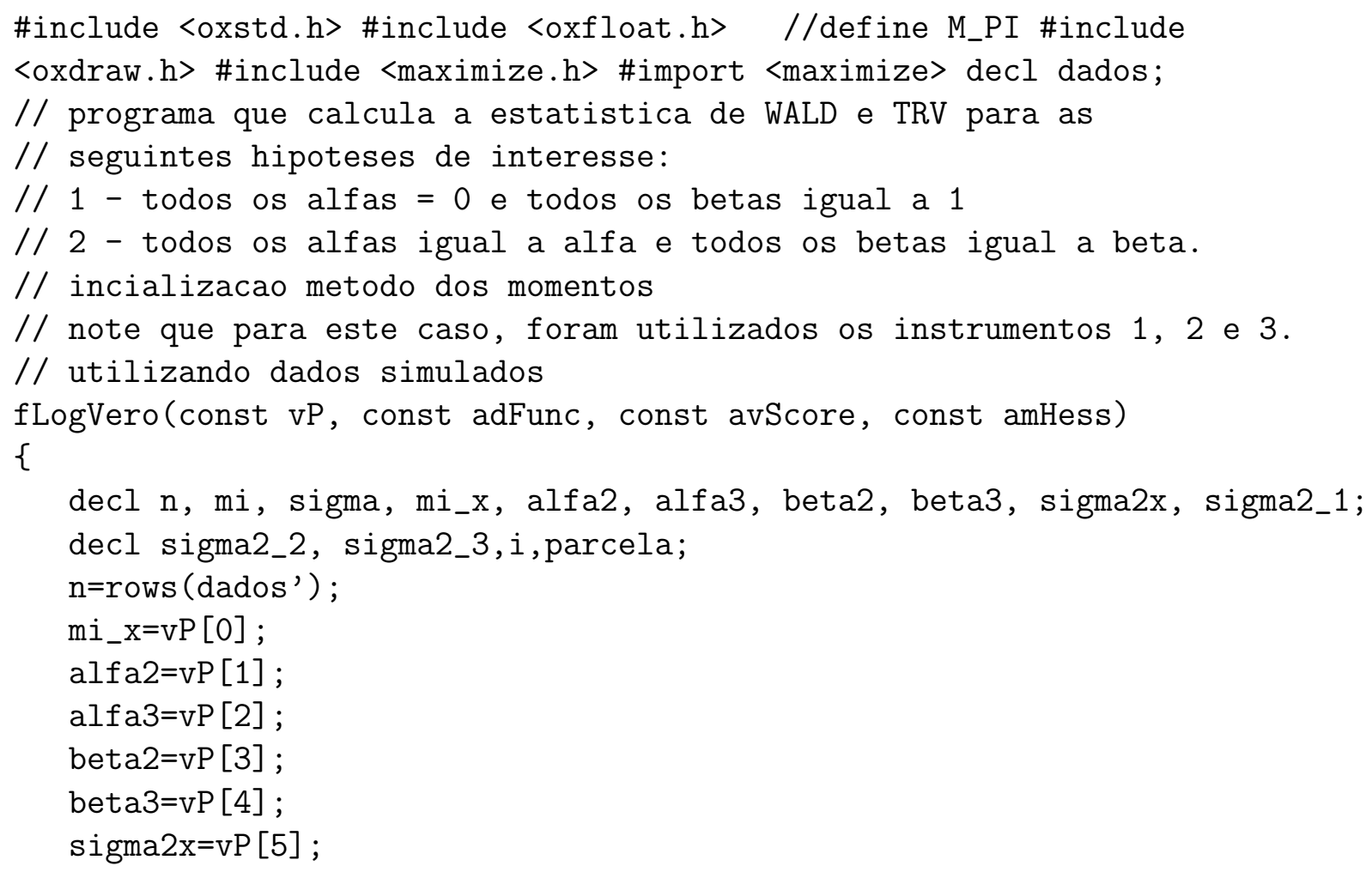




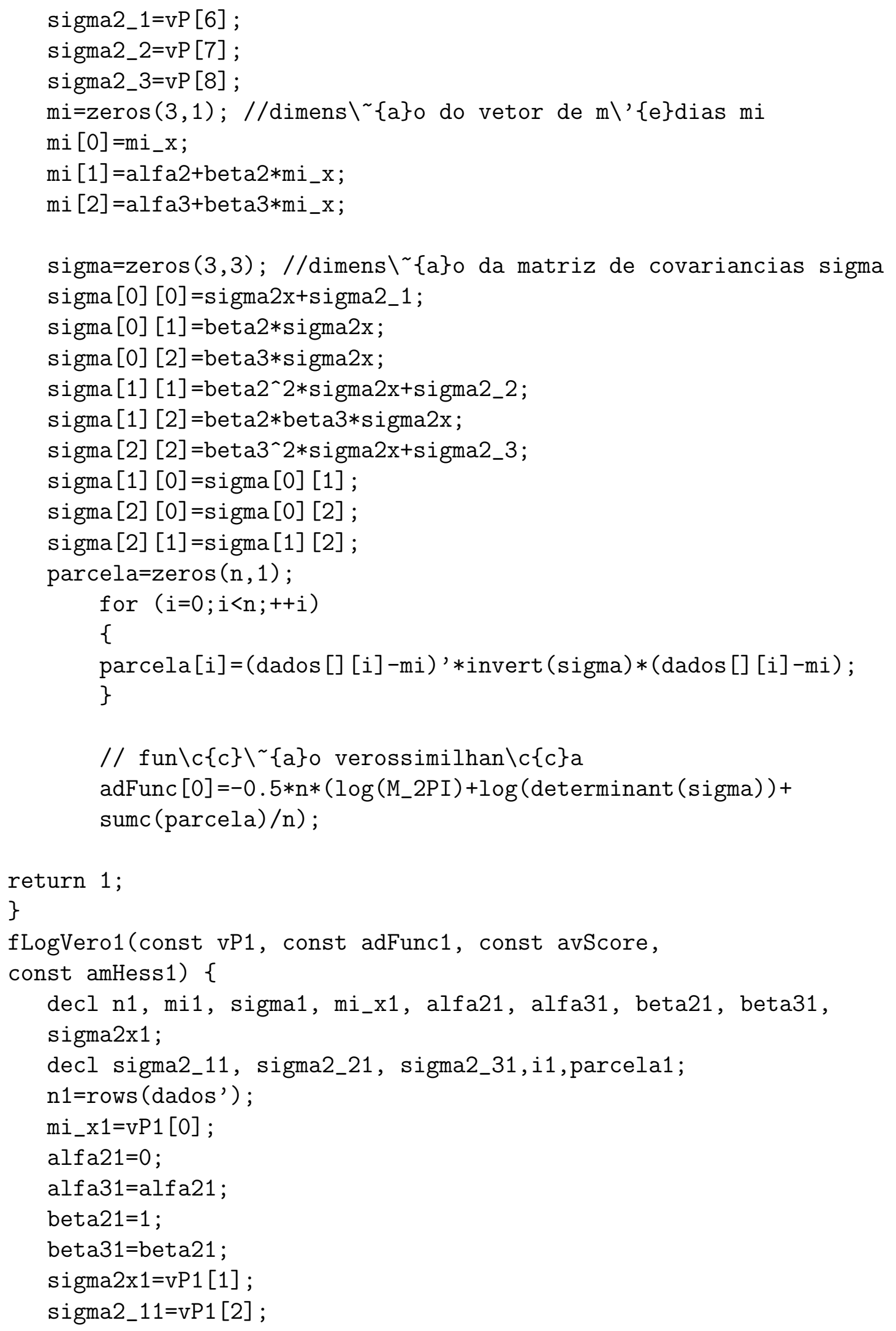




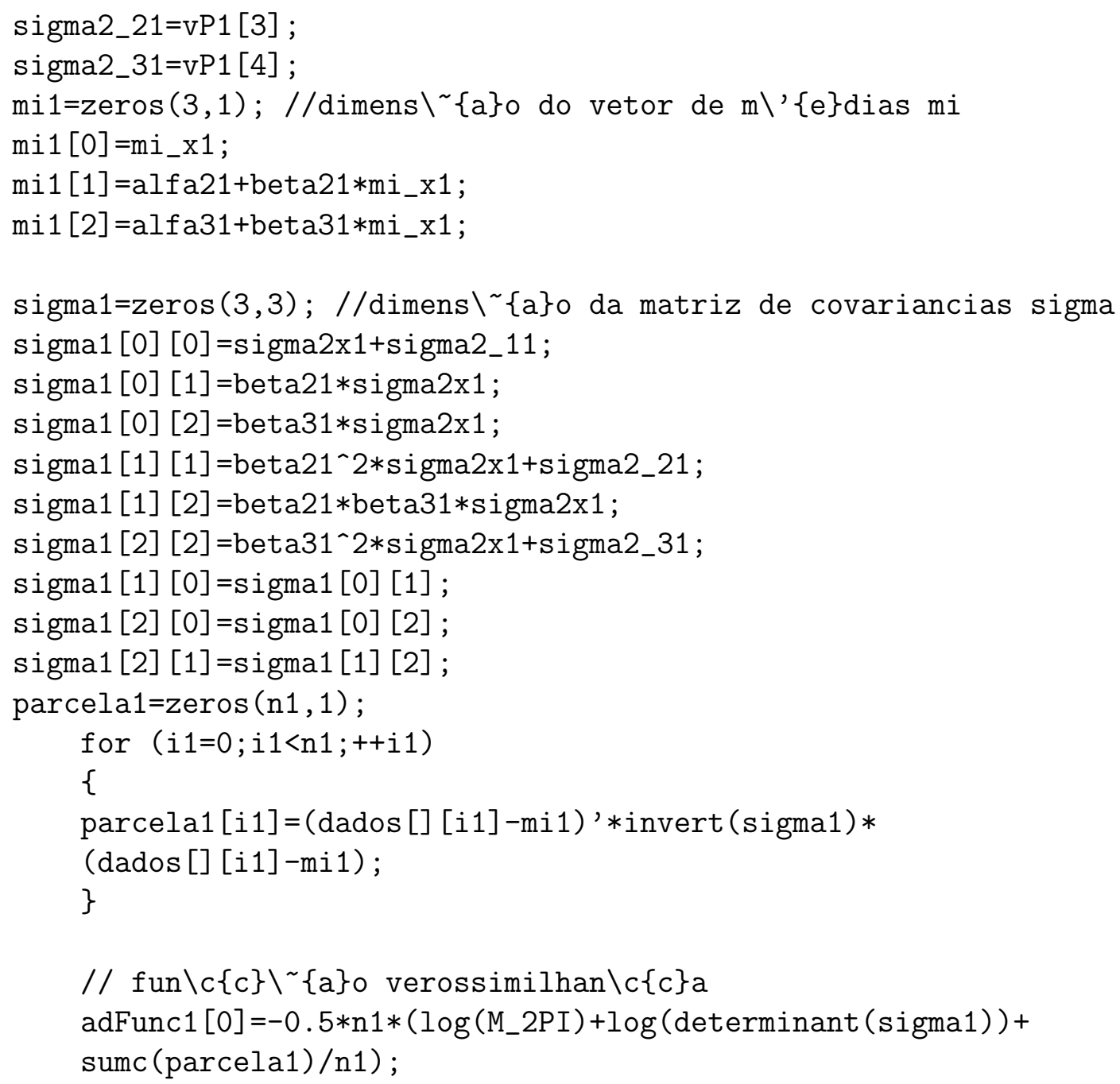




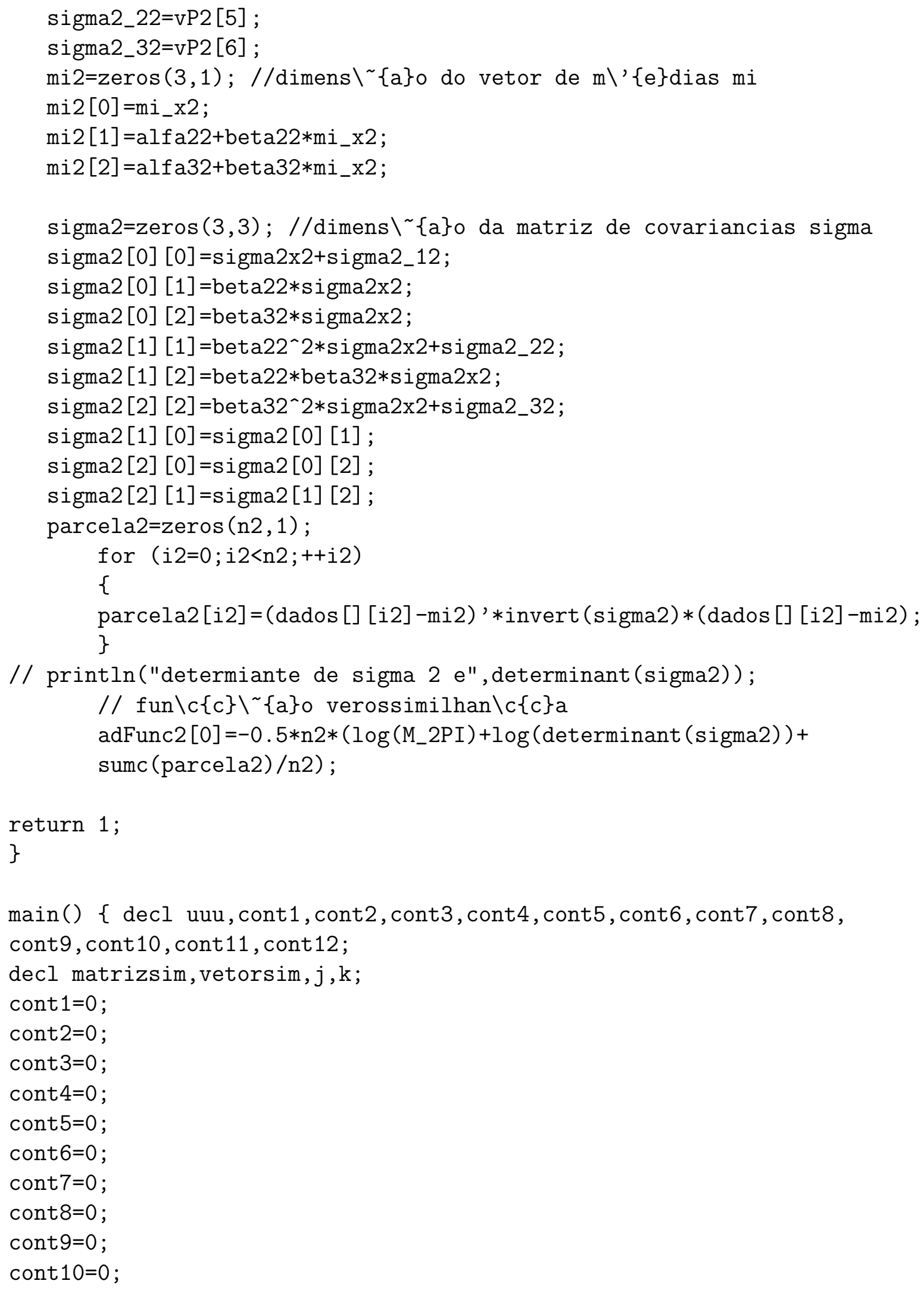




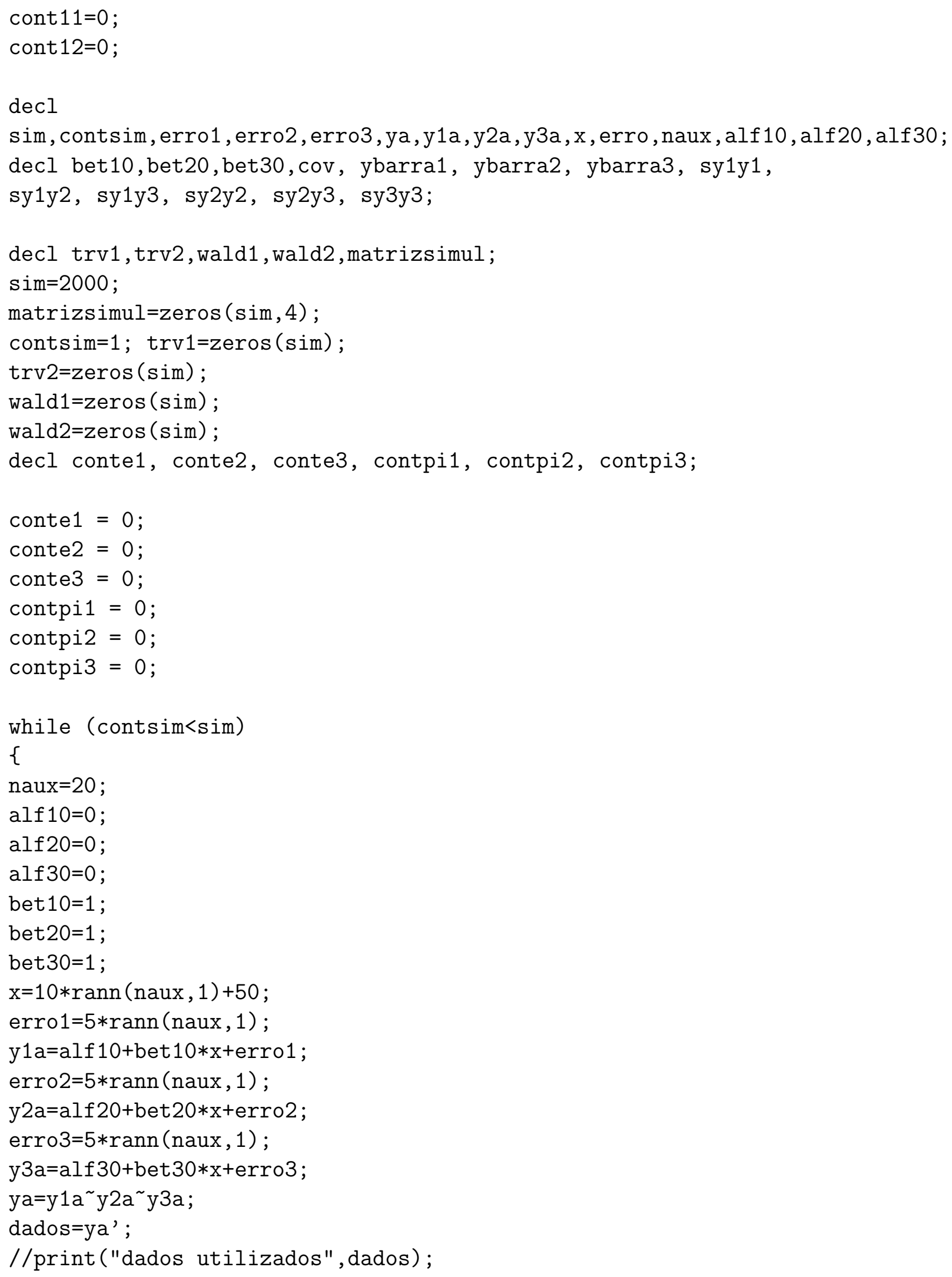


cov=variance (dados' $)$;

// matriz da covariancia das observa $\backslash c\{c\} \backslash \sim\{0\}$ es

sy1y1=cov [0] [0];

sy1y $2=\operatorname{cov}[0][1]$;

sy1y3 $=\operatorname{cov}[0][2]$;

sy $2 \mathrm{y} 2=\operatorname{cov}[1][1]$;

$\operatorname{sy} 2 \mathrm{y} 3=\operatorname{cov}[1][2]$;

sy3y3=cov [2] [2];

ybarra1=meanr (dados [0] [] );

ybarra2=meanr $(\operatorname{dados}[1][])$;

ybarra3=meanr $(\operatorname{dados}[2][])$;

$/ /$ estimadores sem restri $\backslash c\{c\} \backslash \sim\{0\}$ es

decl mi_xMV=ybarra1;

decl sigma2xMV=sy1y2*sy $1 \mathrm{y} 3 /$ sy $2 \mathrm{y} 3$;

decl beta2MV=sy1y2/sigma2xMV;

decl beta3MV=sy1y3/sigma2xMV;

decl alfa2MV=ybarra2-beta2MV*mi_xMV;

decl alfa3MV=ybarra3-beta3MV*mi_xMV;

decl sigma2_1MV=sy1y1-sigma2xMV;

decl sigma2_2MV=sy2y2-beta2MV^2*sigma2xMV;

decl sigma2_3MV=sy3y3-beta3MV^2*sigma2xMV;

//println("METODO DE MAXIMO VEROSSIMILHANCA");

$/ / \operatorname{println}\left(" m i \_x(s / r)=", m i \_x M V\right)$;

// println("ALFA2", alfa2MV);

// println("ALFA3", alfa3MV);

// println("BETA2", beta2MV);

// println("BETA3", beta3MV);

// println ("SIGMA2X", sigma2XMV);

// println("SIGMA1", sigma2_1MV);

// println("SIGMA2", sigma2_2MV);

// println("SIGMA3", sigma2_3MV);

decl teta=zeros $(9,1)$;

teta [0] =mi_xMV;

teta $[1]=a l f a 2 M V$;

teta [2] =alfa3MV; 
teta $[3]=$ beta2MV;

teta $[4]=$ beta3MV;

teta $[5]=$ sigma $2 x M V$;

teta $[6]=$ sigma2_1MV;

teta $[7]=$ sigma2_2MV;

teta $[8]=$ sigma2_3MV;

decl func;

fLogVero (teta, \&func, 0,0 );

// println("valores iniciais dos parametros $\mathbf{s} \backslash \sim\{a\} \circ "$, teta);

decl n=rows (dados');

$/ /$ println("o valor da fun $\backslash c\{c\} \backslash \sim\{a\} 0$ no ponto teta e:",func);

decl mhess=unit (9);

MaxBFGS (fLogVero, \&teta, \&f unc , 0,1 );

$/ /$ println("ponto de $\mathrm{m} \backslash$ ' $\{\mathrm{a}\} \mathrm{ximo",} \mathrm{teta);}$

// println("valor $\mathrm{m} \backslash$ ' $\{a\} x i m o$ da fun $\backslash c\{c\} \backslash \sim\{a\} o ", n * f u n c)$;

Num2Derivative (fLogVero, teta, \&mhess);

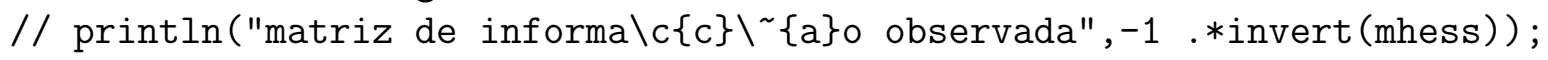

// println("matriz de covariancia observada=",-1 .*mhess);

decl teta1=zeros $(5,1)$;

teta1 [0] =mi_xMV;

teta1 [1] =sigma2xMV;

teta1 [2] =sigma2_1MV;

teta1 [3] =sigma2_2MV;

teta1 [4] =sigma2_3MV;

decl func1; fLogVero1(teta1,\&func1,0,0);

// println("valores iniciais dos parametros $s \backslash \sim\{a\} 0 "$ "teta1);

decl n1=rows (dados');

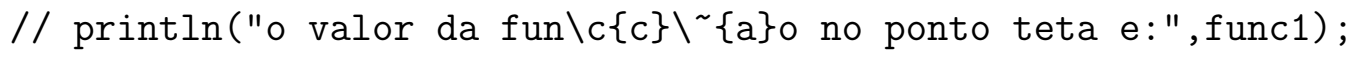

decl mhess1=unit(5);

MaxBFGS (fLogVero1, \&teta1, \&func1, 0, 1) ;

$/ /$ println("ponto de $\mathrm{m} \backslash$ '\{a\}ximo", teta1);

//println("valor $m \backslash$ ' $\{a\} x i m o$ da fun $\backslash c\{c\} \backslash \sim\{a\} o ", n 1 * f u n c 1)$;

Num2Derivative (fLogVero1, teta1, \&mhess1);

$/ /$ println("matriz de informa $\backslash c\{c\} \backslash \sim\{a\} 0$ observada",-1 .*invert(mhess1));

// println("matriz de covariancia observada=",-1 .*mhess1);

decl trv1,trv2; trv1=func-func1; 
// println("o valor do trv1 e:",trv1);

decl teta2=zeros $(7,1)$;

teta2 [0] =mi_xMV;

teta2 [1] =alfa2MV;

teta2 [2] =beta2MV;

teta2 [3] = sigma2xMV;

teta2 [4] =sigma2_1MV;

teta2 [5] =sigma2_2MV;

teta2 [6] =sigma2_3MV;

decl func2; fLogVero2(teta2, \&func2,0,0);

// println("valores iniciais dos parametros $s \backslash \sim\{a\} \circ "$,teta2);

decl n2=rows (dados');

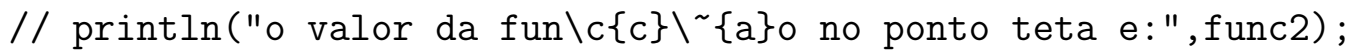

decl mhess2=unit (7);

MaxBFGS ( $f$ LogVero2, \&teta2, \&func2, 0,1 );

$/ /$ println("ponto de $\mathrm{m} \backslash$ ' $\{\mathrm{a}\} \mathrm{ximo}$, teta2);

// println("valor $m \backslash '\{a\} x i m o$ da fun $\backslash c\{c\} \backslash \sim\{a\} o ", n 2 * f u n c 2)$;

Num2Derivative (fLogVero2, teta2, \&mhess2);

$/ /$ println("matriz de informa $\backslash c\{c\} \backslash \sim\{a\} 0$ observada", -1 .*invert(mhess 2$)$ );

// println("matriz de covariancia observada=",-1 .*mhess2);

trv2=func-func2;

// println("o valor do trv2 e:",trv2);

decl y1,y2,y3; y1=submat (dados' $, 0, \mathrm{n}, 0,0)$;

$/ /$ println (y1);

y2=submat (dados' $, 0, \mathrm{n}, 1,1)$;

$/ /$ println (y2);

y3=submat (dados' $, 0, \mathrm{n}, 2,2)$;

$/ /$ println (y3);

// metodo dos momentos.

decl mix, alfa1, alfa2, alfa3, beta1, beta2, beta3, vsigma, sigma1;

decl sigma2, sigma3, sigma2x, valfa, vbeta;

$\operatorname{mix}=\operatorname{meanc}(\mathrm{y} 1)$;

alfa1=0;

alfa2=meanc (y2) -mix* ( (sumc $($ y2 . *y3)/n-meanc (y2)*meanc (y3))/

( $\operatorname{sumc}(\mathrm{y} 1 . * \mathrm{y} 3) / \mathrm{n}-\mathrm{mix} * \operatorname{meanc}(\mathrm{y} 3)))$;

alfa3=meanc $(y 3)-\operatorname{mix} *((\operatorname{sumc}(y 2 . * y 3) / n-m e a n c(y 2) *$ meanc $(y 3)) /$

( $\operatorname{sumc}(\mathrm{y} 1 . * \mathrm{y} 2) / \mathrm{n}-\mathrm{mix} * \operatorname{meanc}(\mathrm{y} 2)))$; 


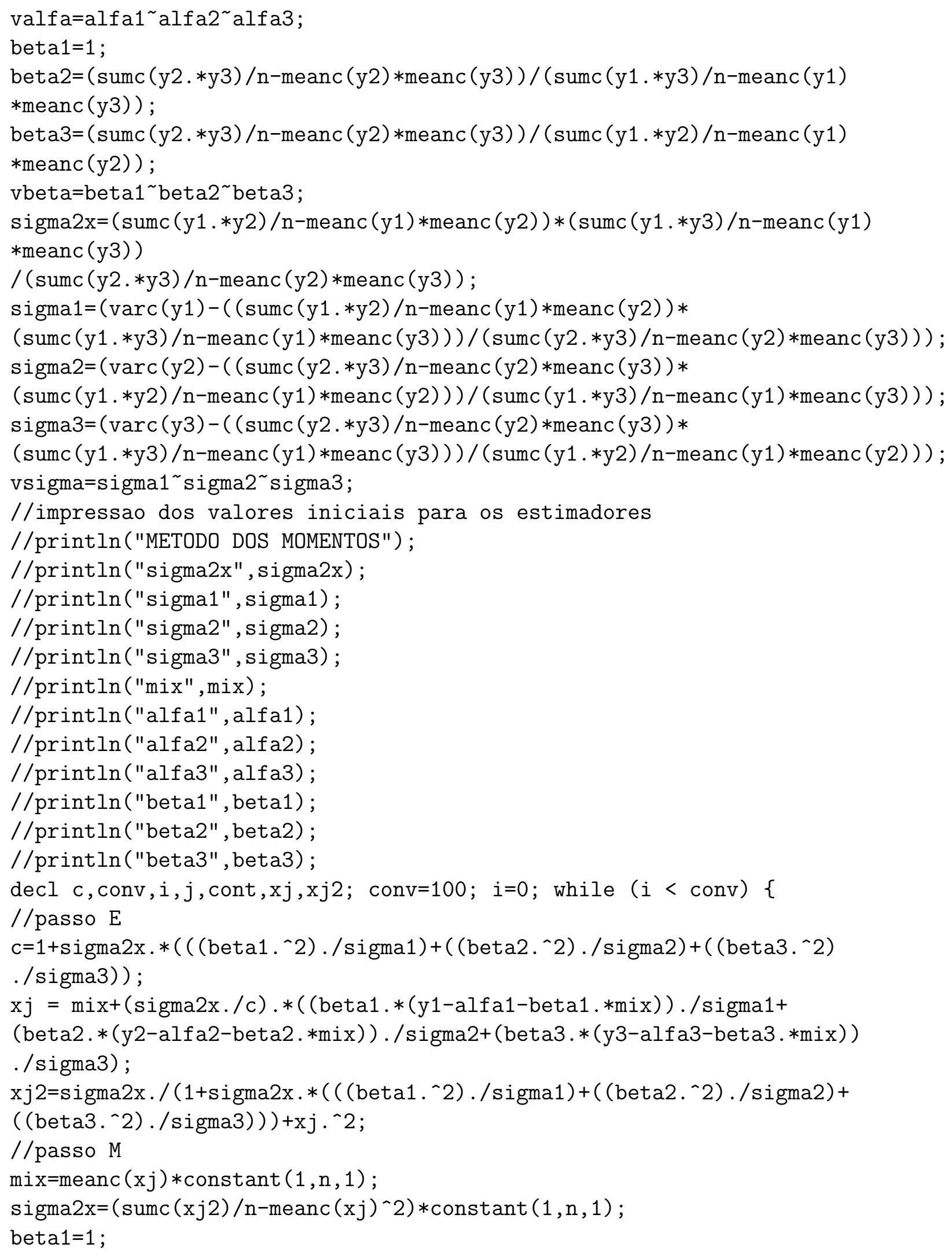




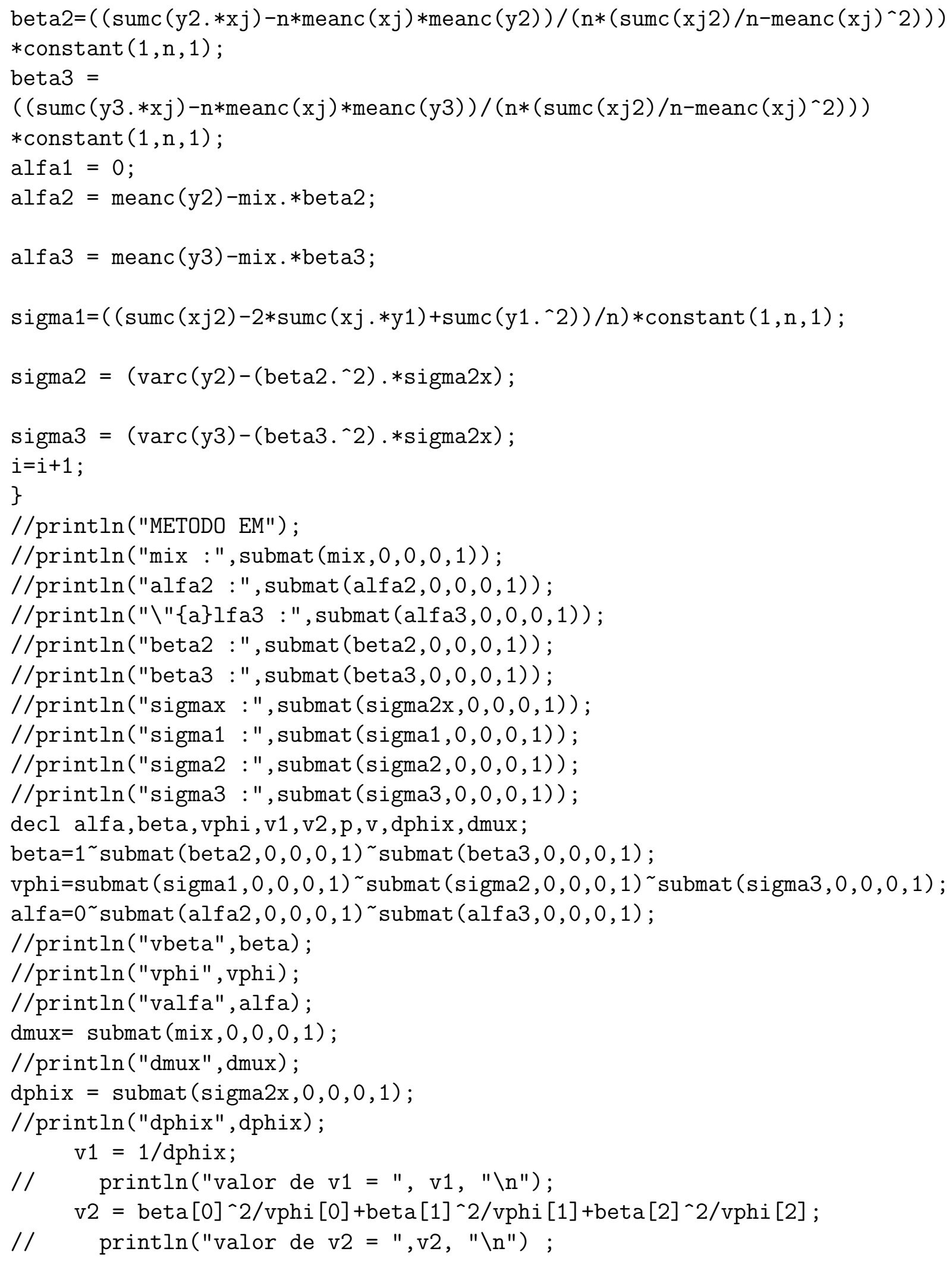




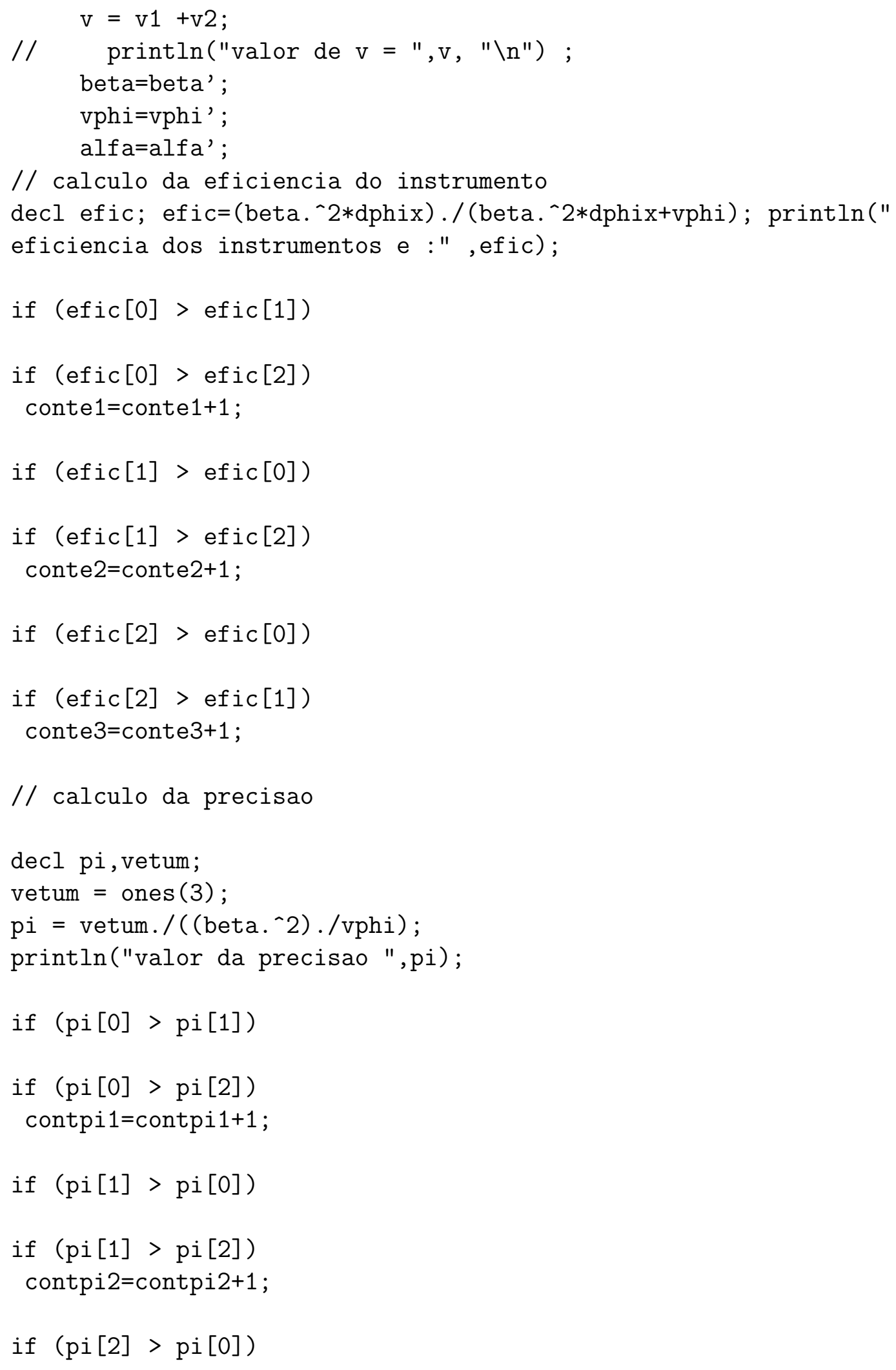




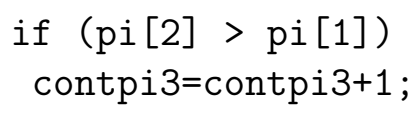

// calculo do estimador de maximo verossimilhanca da precisao decl emvpi;

emvpi $=\left(\right.$ beta. $\left.{ }^{\wedge} 2\right)$./vphi;

println(" valor do estimador de maximo verossimilhanca da precisao : ",emvpi);

// calculo das submatrizes que comp $\backslash \sim\{0\}$ e a matriz de informa $\backslash c\{c\} \backslash \sim\{a\} o$ de fishe decl num, Imixmix, Imixalfak, Imixbetak;

decl Imixsigmax, Imixsigma1, Imixsigmak, Ialfakalfak;

decl Ialfakalfal, Ialfakbetal, Ialfakbetak, Ibetakbetak;

decl Ialfaksigmax, Ialfaksigma1, Ialfaksigmal;

decl Ibetakbetal, Ibetaksigmax, Ibetaksigma1, Ibetaksigmak;

decl Ibetaksigmal, Isigmaxsigmax, Isigmaxsigma1;

decl Isigmaxsigmak, Isigma1sigma1, Isigma1sigmak;

decl Isigmaksigmak, Isigmaksigmal, iaux;

num $=20$;

$\mathrm{p}=2$;

$\operatorname{Imixmix}=\operatorname{num} *(1-1 /(\operatorname{dphix} * v)) / \operatorname{dphix}$;

Imixalfak $=(\operatorname{beta}[1:] . / \operatorname{vphi}[1:]) *($ num $/(\operatorname{v} * \operatorname{dphix}))$;

Imixbetak $=$ num*beta $[1:] * \operatorname{dmux} . /(\mathrm{v} * \operatorname{vphi}[1:] * \operatorname{dphix})$;

Imixsigmax $=0$;

Imixsigma1 $=0$;

Ialfakalfak $=\operatorname{num} *(1-\operatorname{beta}[1:] . * \operatorname{beta}[1:] . /(\operatorname{vphi}[1:] * \mathrm{v})) . / \operatorname{vphi}[1:]$;

Imixsigmak $=\operatorname{zeros}(\mathrm{p}, 1)$;

Ialfaksigmax $=\operatorname{zeros}(\mathrm{p}, 1)$;

Ialfaksigma1 $=\operatorname{zeros}(p, 1)$;

Ialfaksigmal $=\operatorname{zeros}(\mathrm{p}, \mathrm{p})$;

Ialfakalfal $=-$ num $*\left(\operatorname{beta}[1:] * \operatorname{beta}[1:]^{\prime}\right) . /\left(\mathrm{v} * \operatorname{vphi}[1:] * \operatorname{vphi}[1:]^{\prime}\right)$; for $(i=0 ; i<p ;++i)$

Ialfakalfal [i] [i] = Ialfakalfak[i];

Ialfakbetal $=-$ num $*\left(\operatorname{beta}[1:] * \operatorname{beta}[1:]^{\prime}\right) * \operatorname{dmux} . /(\mathrm{v} * \operatorname{vphi}[1:] *$ vphi[1:]') ; 
Ialfakbetak $=-$ num*beta $[1:] . * \operatorname{beta}[1:] * \operatorname{dmux} . /(\operatorname{v} * \operatorname{vph} i[1:] * * \operatorname{vphi}[1:])$ +num*dmux./(vphi [1:]);

for $(i=0 ; i<p ;++i)$

Ialfakbetal [i] [i] = Ialfakbetak[i];

Ibetakbetak $=\left(\right.$ num* $\left.*\left(\operatorname{dmux}^{\wedge} 2+\operatorname{dphix}-1 / v\right)\right) . / \operatorname{vphi}[1:]+($ num $* \operatorname{beta}[1:]$.

*beta $\left.[1:] *\left(2 / \mathrm{v}-\operatorname{dphix}-\operatorname{dmux}^{\wedge} 2\right)\right)$./(v*vphi [1:] .*vphi [1:]);

Ibetakbetal $=$ num* $\left(\operatorname{beta}[1:] * \operatorname{beta}[1:]^{\prime}\right) . /\left(\mathrm{v} * \operatorname{vphi}[1:] * \operatorname{vphi}[1:]^{\prime}\right) *$

(2/v-dphix-dmux`2);

for $(i=0 ; i<p ;++i)$

Ibetakbetal [i] [i] = Ibetakbetak [i];

Ibetaksigmax $=($ num*beta $[1:] *(\operatorname{dphix}-1 / v)) . /\left(v^{2} * \operatorname{vphi}[1:] * \operatorname{dphix}{ }^{\wedge} 2\right)$;

Ibetaksigma1 $=-$ num $* \operatorname{beta}[1:] . /\left(v^{\wedge} 2 * \operatorname{vphi}[1:] * \operatorname{vphi}\left[0{ }^{\wedge} 2\right)\right.$;

Ibetaksigmak $=($ num $* \operatorname{beta}[1:] . /(\operatorname{v} * \operatorname{vphi}[1:] . * \operatorname{vphi}[1:])) . *$

(1-beta[1:].*beta[1:]./(v*vphi[1:]));

Ibetaksigmal $=-\operatorname{num} *\left(\operatorname{beta}[1:] * \operatorname{beta}[1:]^{\prime} \cdot{ }^{\wedge} 2\right) . /\left(\mathrm{v}^{\wedge} 2 * \operatorname{vphi}[1:]\right.$

* vphi[1:]'.`2);

for $(i=0 ; i<p ;++i)$

Ibetaksigmal [i] [i] = Ibetaksigmak [i];

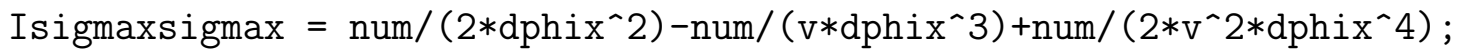

Isigmaxsigma1 $=$ num $/\left(2 * v^{\wedge} 2 * \operatorname{dphix}{ }^{\wedge} 2 * \operatorname{vphi}[0] \wedge 2\right)$;

Isigmaxsigmak $=$ num $*(\operatorname{beta}[1:] . * \operatorname{beta}[1:]) . /\left(2 * v^{\wedge} 2 * \operatorname{dphix}{ }^{\wedge} * \operatorname{vphi}[0]^{\wedge} 2\right)$;

Isigma1sigma1 = num/(2*vphi [0]^2)-num/(v*vphi [0]^3)+num/(2*v^2*vphi [0]^4);

Isigma1sigmak $=$ num* $(\operatorname{beta}[1:] . * \operatorname{beta}[1:]) . /\left(2 * \mathrm{v}^{\wedge} 2 * \operatorname{vphi}[0]^{\wedge} 2 * \operatorname{vphi}\left[1:{ }^{\wedge}{ }^{\wedge} 2\right)\right.$;

Isigmaksigmak $=$ num. $/(2 * \operatorname{vphi}[1:] . * \operatorname{vphi}[1:])-\operatorname{num} *(\operatorname{beta}[1:]$.

*beta[1:]) ./(v*vphi [1:] .*vphi [1:] .*vphi [1:])+num*(beta[1:] .*beta[1:]

* beta $[1:]$.

*beta[1:])./(2*v^2*vphi [1:] .*vphi [1:] **vphi [1:] **vphi [1:]);

Isigmaksigmal $=$ num* $(\operatorname{beta}[1:] . \wedge 2) *(\operatorname{beta}[1:] . \wedge 2)^{\prime} \cdot /\left(2 * \mathrm{v}^{\wedge} 2 *(\operatorname{vphi}[1:] . \wedge 2)\right.$

*(vphi $\left.\left.[1:] .{ }^{\wedge} 2\right)^{\prime}\right)$;

for $(i=0 ; i<p ;++i)$

Isigmaksigmal [i] [i] = Isigmaksigmak [i];

// concatena $\backslash c\{c\} \backslash \sim\{a\} o$ das submatrizes

decl Imix, Ialfa, Ibeta, Isigmax, Isigma1, Isigma;

Imix = Imixmix | Imixalfak | Imixbetak | Imixsigmax | Imixsigma1

I Imixsigmak;

Ialfa = Imixalfak'|Ialfakalfal|Ialfakbetal|Ialfaksigmax'|Ialfaksigma1'

I Ialfaksigmal;

Ibeta = Imixbetak'|Ialfakbetal'|Ibetakbetal $\mid$ Ibetaksigmax' $\mid$ Ibetaksigma1'

I Ibetaksigmal';

Isigmax = Imixsigmax $\mid$ Ialfaksigmax | Ibetaksigmax | Isigmaxsigmax |

Isigmaxsigma1 | Isigmaxsigmak;

Isigma1 = Imixsigma1 | Ialfaksigma1 | Ibetaksigma1 | Isigmaxsigma1 |

Isigma1sigma1 | Isigma1sigmak; 
Isigma = Imixsigmak'| Ialfaksigmal | Ibetaksigmal | Isigmaxsigmak' |

Isigma1sigmak' | Isigmaksigmal;

$/ /$ matriz de informa $\backslash c\{c\} \backslash \sim\{a\} 0$ de fisher

decl I;

$$
\text { I }=\text { Imix } \sim \text { Ialfa } \sim \text { Ibeta } \sim \text { Isigmax } \sim \text { Isigma1 } \sim \text { Isigma; }
$$

// println("valor de $I=$ ", I, "\n") ;

// matriz inversa da informa $\backslash c\{c\} \backslash \sim\{a\} \circ$ de fisher

decl Inv;

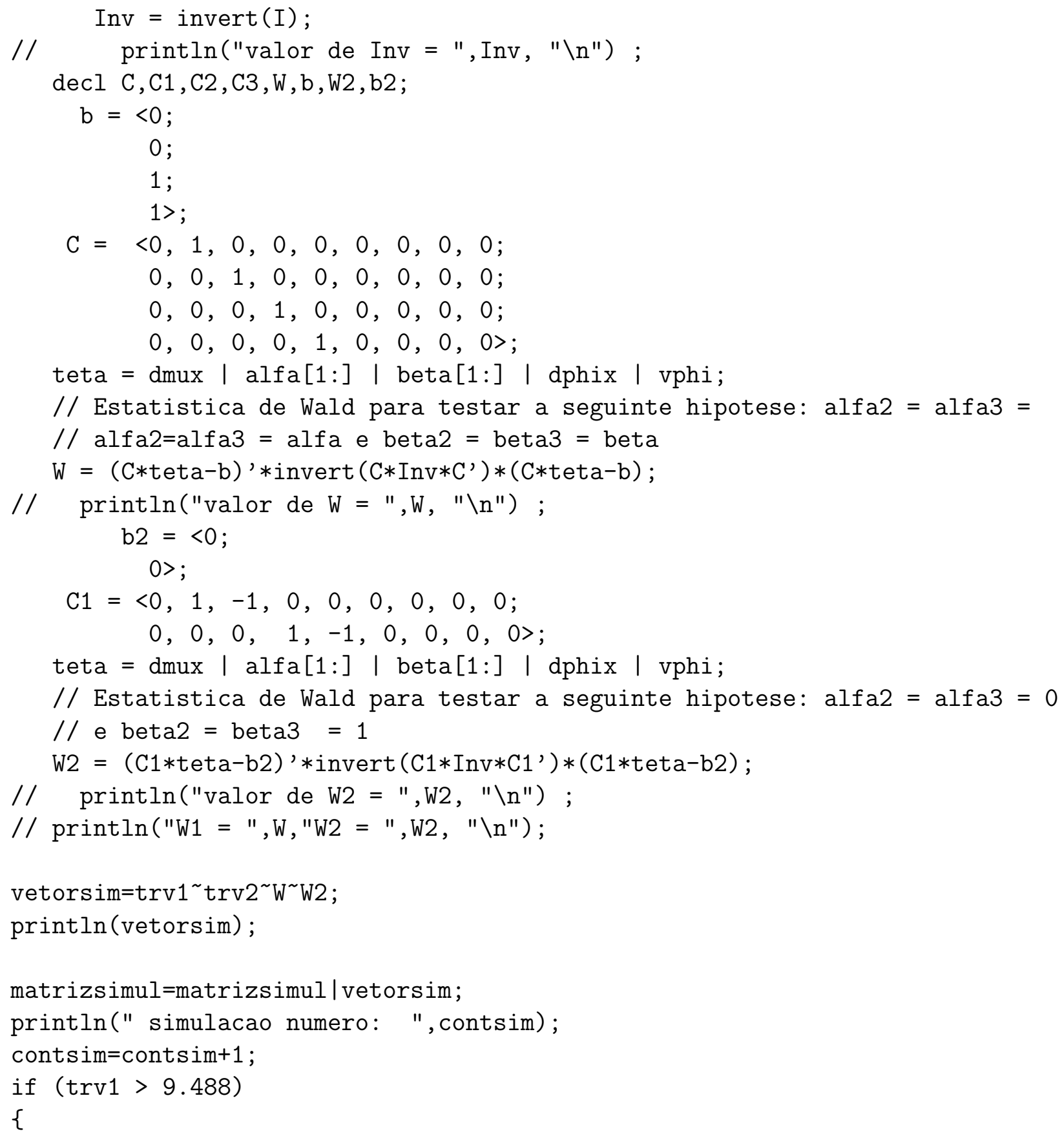




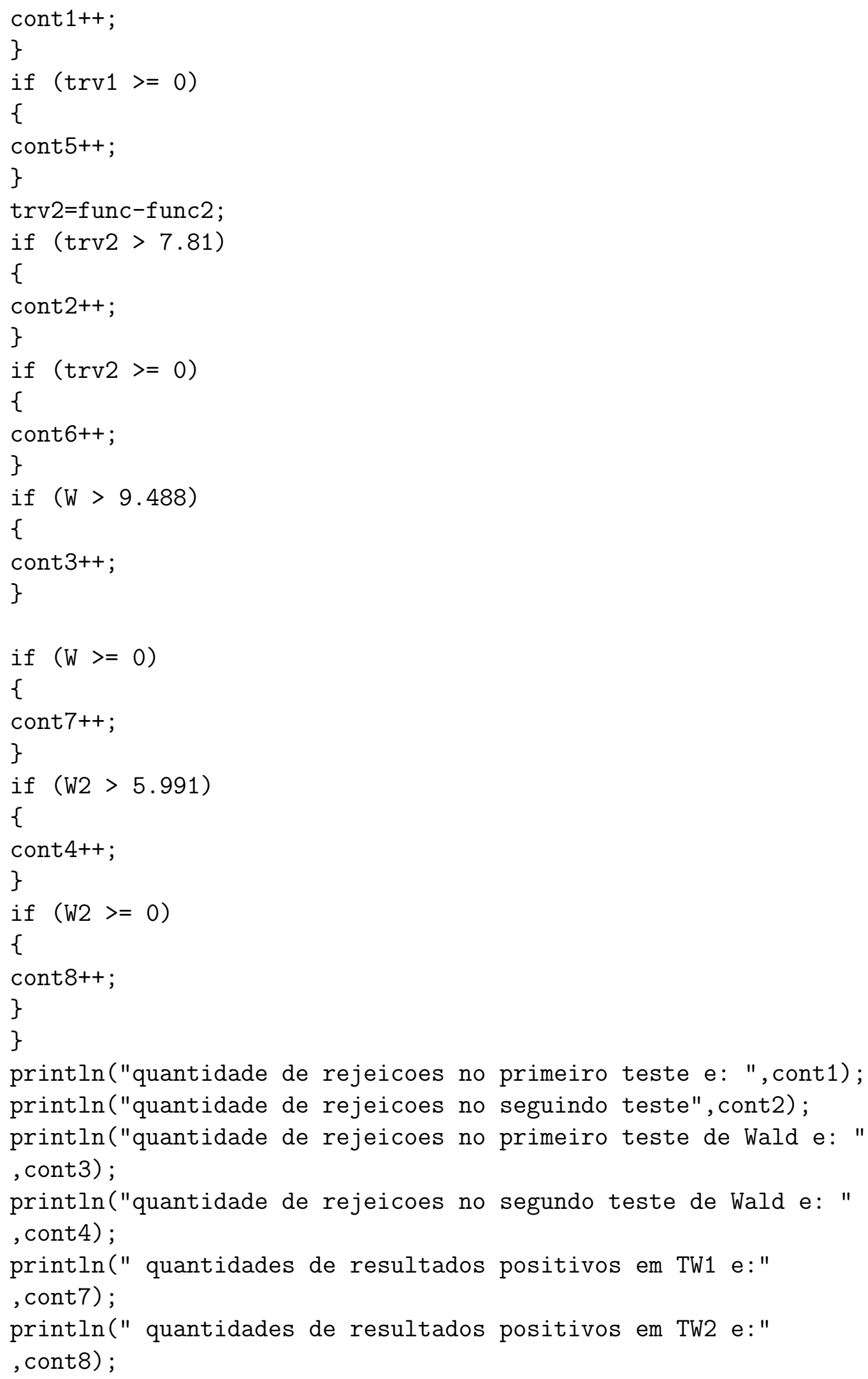




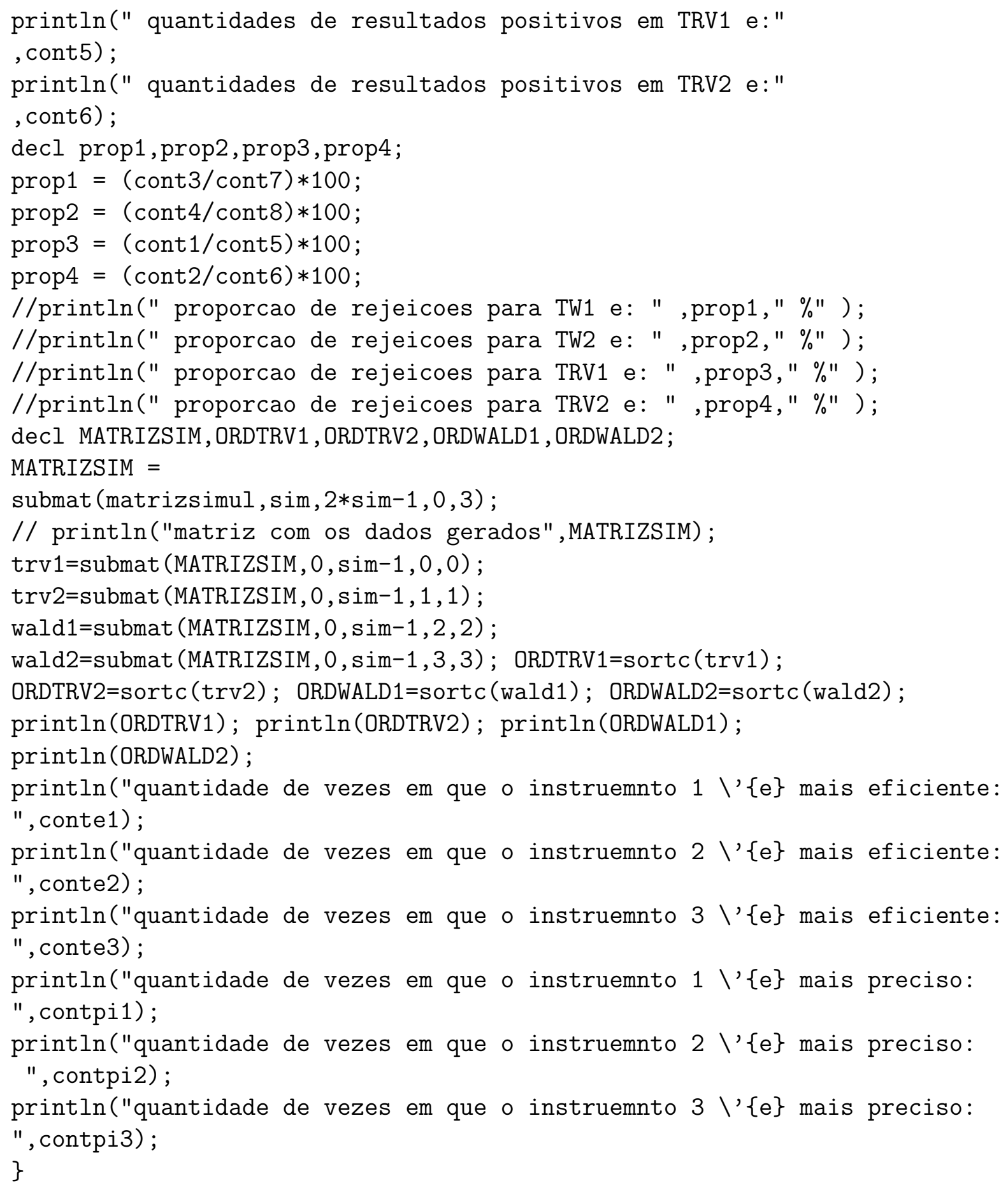

Segundo Programa.

\#include <oxstd.h> \#include <oxfloat.h> //define M_PI \#include <oxdraw.h> \#include <maximize.h> \#import <maximize> decl dados; 
// programa que calcula a estatistica de WALD e TRV para as

// seguintes hipoteses de interesse:

$/ / 1$ - todos os alfas $=0$ e todos os betas igual a 1

// 2 - todos os alfas igual a alfa e todos os betas igual a beta.

// incializacao metodo dos momentos

// note que para este caso, foram utilizados os instrumentos 1,2 e 3 .

fLogVero(const VP, const adFunc, const avScore, const amHess)

\{

decl n, mi, sigma, mi_x, alfa2, alfa3, beta2, beta3, sigma2x, sigma2_1;

decl sigma2_2, sigma2_3,i,parcela;

$\mathrm{n}=\operatorname{rows}\left(\right.$ dados' $\left.^{\prime}\right)$;

$\mathrm{mi} \_\mathrm{x}=\mathrm{vP}[0]$;

alfa2 $=v P[1]$;

alfa3 $=v P[2]$;

beta2 $=\mathrm{vP}[3]$;

beta3 $=v P[4]$;

sigma $2 x=v P[5]$;

sigma2_1=vP $[6]$;

sigma2_2=vP $[7]$;

sigma2_3=vP $[8]$;

$\mathrm{mi}=\operatorname{zeros}(3,1) ; / /$ dimens $\backslash \sim\{a\} o$ do vetor de $m \backslash$ ' $\{$ e\}dias $\mathrm{mi}$

$\operatorname{mi}[0]=\mathrm{mi} \mathrm{x}_{\text {; }}$

mi $[1]=a l f a 2+$ beta2 $*$ mi_x;

$\operatorname{mi}[2]=a l f a 3+$ beta3*mi_x;

sigma=zeros $(3,3)$; //dimens $\backslash \sim$ a $\}$ o da matriz de covariancias sigma

sigma [0] [0] =sigma2x+sigma2_1;

sigma [0] [1] =beta $2 *$ sigma $2 x$;

sigma [0] [2] =beta3*sigma $2 x$;

sigma [1] [1] =beta2^2*sigma2x+sigma2_2 ;

sigma [1] [2] =beta2*beta3*sigma $2 x$;

sigma [2] [2] =beta3^2*sigma2x+sigma2_3;

sigma [1] [0] =sigma [0] [1] ;

sigma [2] [0] =sigma [0] [2] ;

sigma [2] [1] =sigma [1] [2] ;

$\operatorname{parcela}=\operatorname{zeros}(\mathrm{n}, 1)$;

for $(i=0 ; i<n ;++i)$

\{

parcela $[i]=(\operatorname{dados}[][i]-m i)$ '*invert (sigma)*

(dados [] [i]-mi);

\}

$/ /$ fun $\backslash c\{c\} \backslash \sim\{a\} o$ verossimilhan $\backslash c\{c\} a$ 
$\operatorname{adFunc}[0]=-0.5 * n *\left(\log \left(M_{2} 2 P I\right)+\log (\operatorname{determinant}(\right.$ sigma $))+$ sumc (parcela)/n);

return 1;

\}

fLogVero1(const vP1, const adFunc1, const avScore, const amHess1)

\{

decl n1, mi1, sigma1, mi_x1, alfa21, alfa31, beta21, beta31, sigma2x1;

decl sigma2_11, sigma2_21, sigma2_31,i1,parcela1;

$\mathrm{n} 1=\operatorname{rows}\left(\right.$ dados $\left.^{\prime}\right)$;

mi_x1=vP1[0];

alfa21=0;

alfa31=alfa21;

beta21=1;

beta31=beta21;

sigma $2 \times 1=v P 1[1]$;

sigma2_11=vP1[2];

sigma2_21=vP1[3];

sigma2_31=vP1 [4];

mi1=zeros $(3,1) ; / /$ dimens $\backslash \sim\{a\} o$ do vetor de $m \backslash$ ' $\{$ e\}dias mi

$\operatorname{mi1}[0]=m i \_x 1$;

$\operatorname{mi1}[1]=a l f a 21+$ beta21*mi_x1;

$\operatorname{mi1}[2]=a l f a 31+$ beta31*mi_x1;

sigma1=zeros $(3,3) ; / /$ dimens $\backslash \sim\{a\} o$ da matriz de covariancias sigma

sigma1 [0] [0] =sigma2x1+sigma2_11;

sigma1 [0] [1] =beta21*sigma $2 \times 1$;

sigma1 [0] [2] =beta31*sigma2x1;

sigma1 [1] [1] =beta21^2*sigma2x1+sigma2_21;

sigma1 [1] [2] =beta21*beta31*sigma2x1;

sigma1 [2] [2] =beta31^2*sigma2x1+sigma2_31;

sigma1 [1] [0] =sigma1 [0] [1];

sigma1 [2] [0] =sigma1 [0] [2] ;

sigma1 [2] [1] =sigma1 [1] [2] ;

parcela1=zeros $(n 1,1)$;

for $(i 1=0 ; i 1<n 1 ;++i 1)$

\{

parcela1 [i1] $=(\operatorname{dados}[][i 1]-m i 1) ' * \operatorname{invert}($ sigma1 $) *$

(dados [] [i1]-mi1);

\}

$/ /$ fun $\backslash c\{c\} \backslash \sim\{a\} o$ verossimilhan $\backslash c\{c\} a$ 
$\operatorname{adFunc} 1[0]=-0.5 * \mathrm{n} 1 *\left(\log \left(\mathrm{M}_{-} 2 \mathrm{PI}\right)+\log (\operatorname{determinant}(\operatorname{sigma} 1))+\right.$ sumc (parcela1)/n1);

return 1;

\}

fLogVero2(const vP2, const adFunc2, const avScore2, const amHess2) \{

decl n2, mi2, sigma2, mi_x2, alfa22, alfa32, beta22, beta32, sigma2x2;

decl sigma2_12, sigma2_22, sigma2_32,i2,parcela2;

n2=rows (dados');

$\mathrm{mi} \times \mathrm{x} 2=\mathrm{vP} 2[0]$;

alfa22=vP2 [1];

alfa32=alfa22;

beta22=vP2 [2];

beta32=beta22;

sigma $2 \times 2=v P 2[3]$;

sigma2_12=vP2 [4];

sigma2_22=vP2 [5];

sigma2_32=vP2 [6];

mi2=zeros $(3,1) ; / /$ dimens $\backslash \sim\{a\} 0$ do vetor de $m \backslash$ ' $\{$ e $\}$ dias $m i$

$\operatorname{mi2}[0]=\mathrm{mi} \mathrm{x} 2$;

$\operatorname{mi2}[1]=a l f a 22+$ beta $22 * m i \_x 2$;

$\operatorname{mi2}[2]=a l f a 32+$ beta32*mi_x2;

sigma2=zeros $(3,3) ; / /$ dimens $\backslash \sim\{a\} 0$ da matriz de covariancias sigma sigma2 [0] [0] =sigma2x2+sigma2_12;

sigma2 [0] [1] =beta22*sigma $2 \times 2$;

sigma2 [0] [2] =beta32*sigma $2 \times 2$;

sigma2 [1] [1] =beta22^2*sigma2x2+sigma2_22;

sigma2 [1] [2] =beta22*beta32*sigma $2 \times 2$;

sigma2 [2] [2] =beta32^2*sigma2x2+sigma2_32;

sigma2 [1] [0] =sigma2 [0] [1];

sigma2 [2] [0] =sigma2 [0] [2];

sigma2 [2] [1] =sigma2 [1] [2] ;

parcela2=zeros $(\mathrm{n} 2,1)$;

for $(i 2=0 ;$ i $2<\mathrm{n} 2 ;++\mathrm{i} 2)$

\{

parcela2 [i2] $=(\operatorname{dados}[][i 2]-m i 2) ' * i n v e r t(\operatorname{sigma} 2) *(\operatorname{dados}[][i 2]-m i 2)$;

\}

// println("determiante de sigma 2 e", determinant(sigma2));

$/ /$ fun $\backslash c\{c\} \backslash \sim\{a\} o$ verossimilhan $\backslash c\{c\} a$

$\operatorname{adFunc} 2[0]=-0.5 * \mathrm{n} 2 *\left(\log \left(\mathrm{M}_{-} 2 \mathrm{PI}\right)+\log (\operatorname{determinant}(\operatorname{sigma} 2))+\right.$ sumc (parcela2)/n2); 


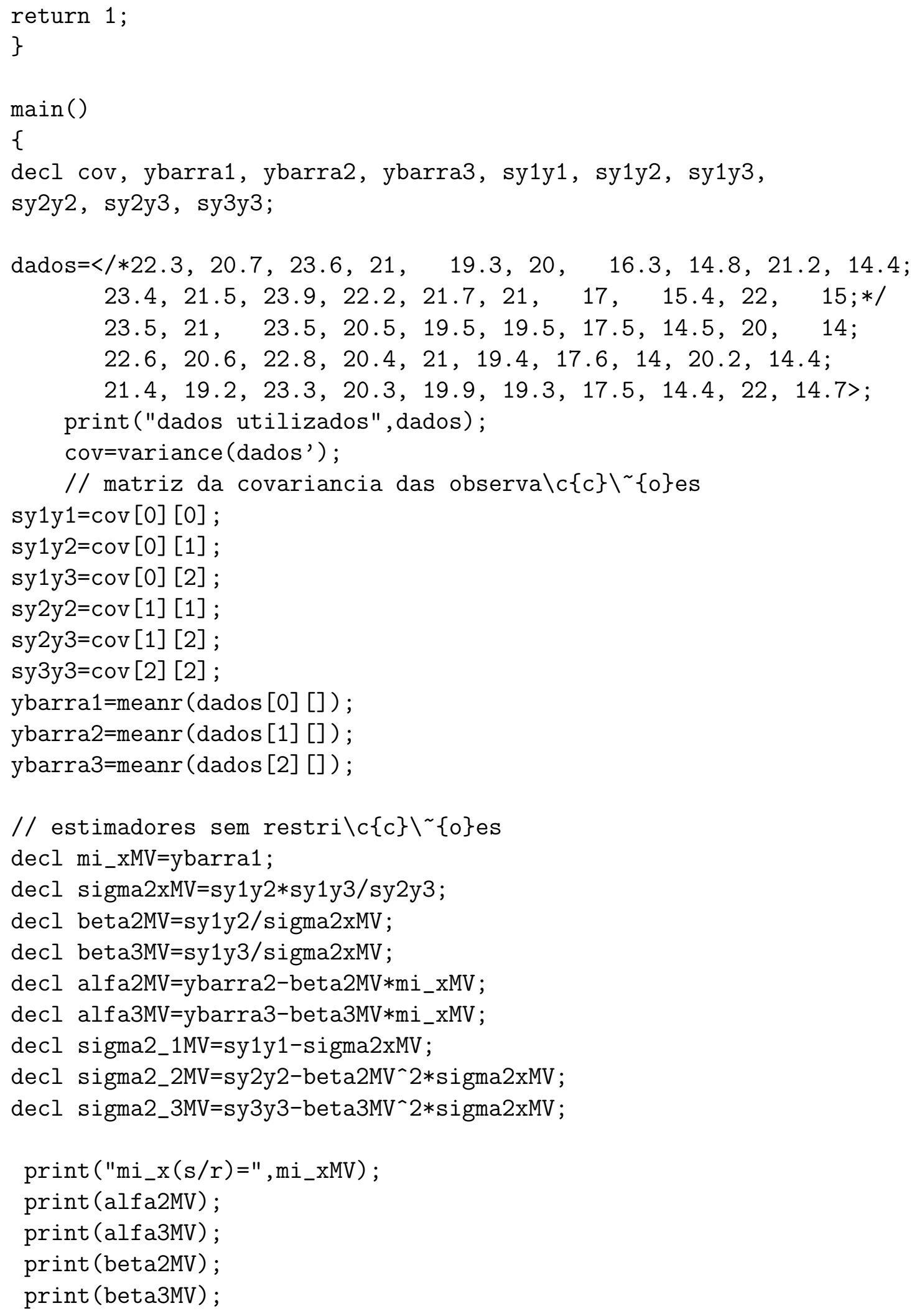




$$
\begin{aligned}
& \text { print (sigma2xMV); } \\
& \text { print (sigma2_1MV); } \\
& \text { print (sigma2_2MV); } \\
& \text { print(sigma2_3MV); }
\end{aligned}
$$

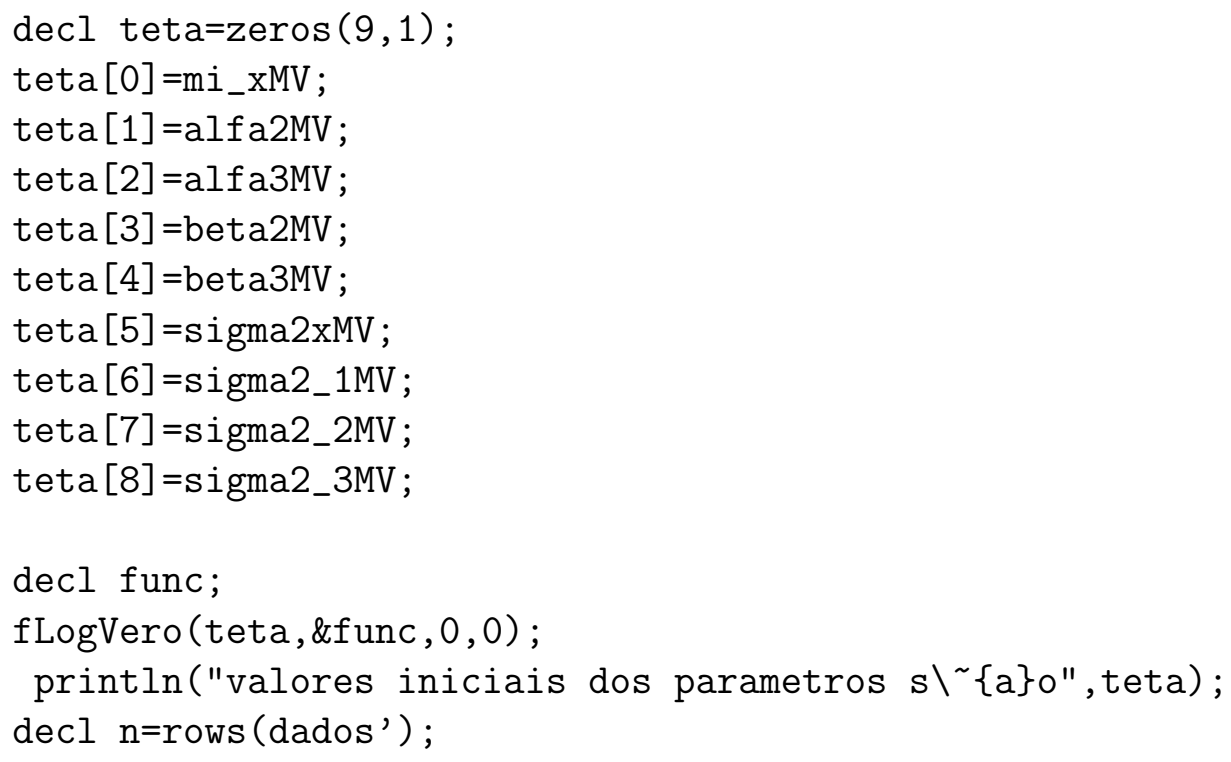




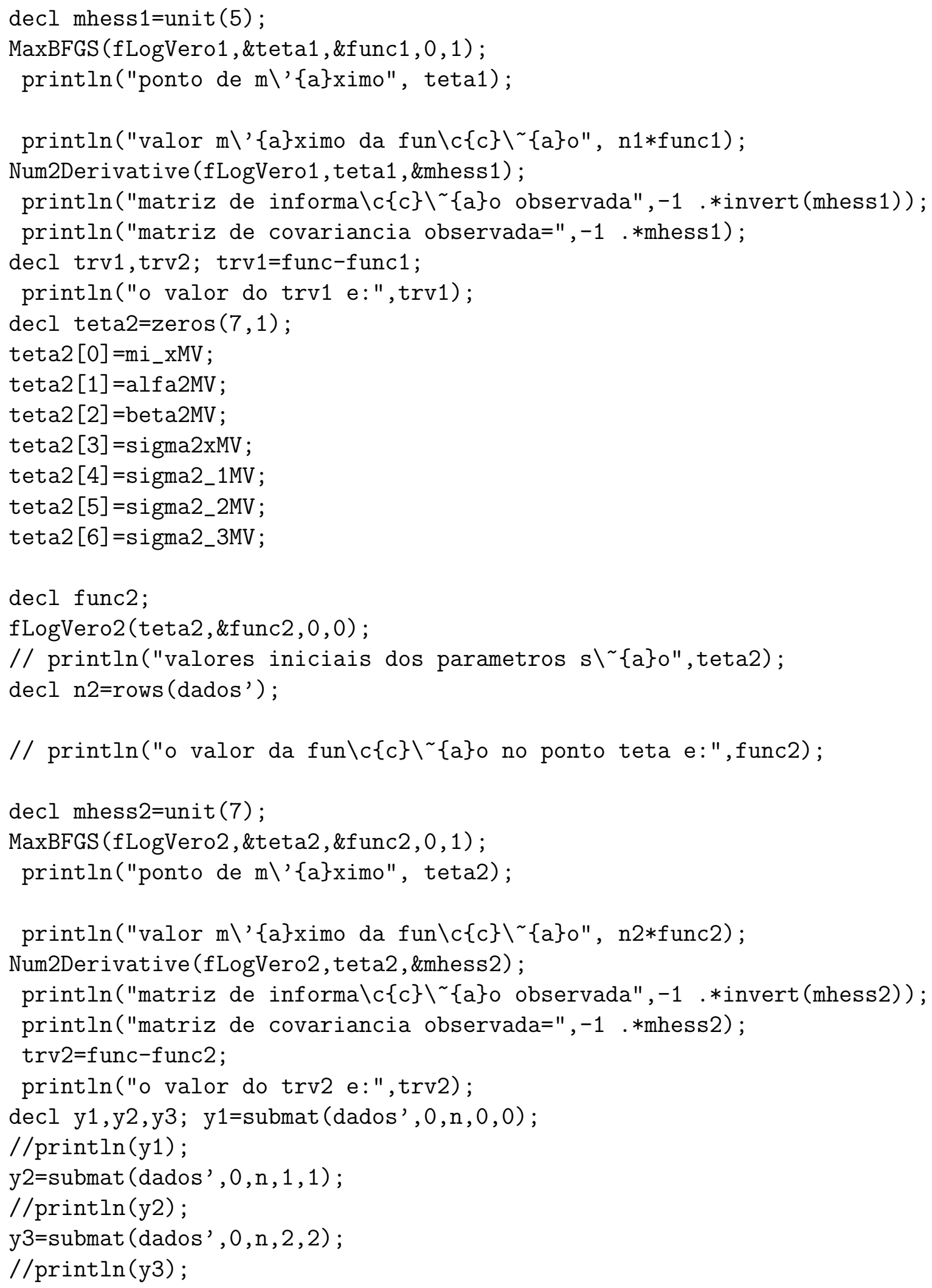


// metodo dos momentos.

decl mix, alfa1, alfa2, alfa3, beta1, beta2, beta3, vsigma;

decl sigma1, sigma2, sigma3, sigma2x, valfa, vbeta;

$\operatorname{mix}=\operatorname{meanc}(\mathrm{y} 1)$;

alfa1=0;

alfa2 $=$ meanc $(y 2)-\operatorname{mix} *((\operatorname{sumc}(\mathrm{y} 2 . * \mathrm{y} 3) / \mathrm{n}-\operatorname{meanc}(\mathrm{y} 2) * \operatorname{meanc}(\mathrm{y} 3)) /(\operatorname{sumc}(\mathrm{y} 1 . * \mathrm{y} 3)$

$/ \mathrm{n}-\mathrm{mix} * \operatorname{meanc}(\mathrm{y} 3)))$;

alfa3=meanc $($ y3) $-\operatorname{mix} *((\operatorname{sumc}($ y2 . *y3) $/ \mathrm{n}-\operatorname{meanc}(\mathrm{y} 2) *$ meanc $(\mathrm{y} 3))$

$/(\operatorname{sumc}(\mathrm{y} 1 . * \mathrm{y} 2) / \mathrm{n}-\mathrm{mix} * \operatorname{meanc}(\mathrm{y} 2)))$;

valfa=alfa1 alfa2 alfa3; beta1=1;

beta2 $=(\operatorname{sumc}(\mathrm{y} 2 . * \mathrm{y} 3) / \mathrm{n}-\operatorname{meanc}(\mathrm{y} 2) *$ meanc $(\mathrm{y} 3)) /(\operatorname{sumc}(\mathrm{y} 1 . * \mathrm{y} 3) / \mathrm{n}-\operatorname{meanc}(\mathrm{y} 1)$

*meanc (y3)) ;

beta3 $=(\operatorname{sumc}(\mathrm{y} 2 . * \mathrm{y} 3) / \mathrm{n}-\operatorname{meanc}(\mathrm{y} 2) *$ meanc $(\mathrm{y} 3)) /($ sumc $(\mathrm{y} 1 . * \mathrm{y} 2) / \mathrm{n}-\mathrm{meanc}(\mathrm{y} 1)$

*meanc (y2)) ;

vbeta=beta1 $\sim$ beta2 $\sim$ beta3;

sigma2x $=(\operatorname{sumc}(\mathrm{y} 1 . * \mathrm{y} 2) / \mathrm{n}-\operatorname{meanc}(\mathrm{y} 1) *$ meanc $(\mathrm{y} 2)) *(\operatorname{sumc}(\mathrm{y} 1 . * \mathrm{y} 3) / \mathrm{n}$

-meanc (y1)*meanc (y3))/( sumc $(\mathrm{y} 2 . * \mathrm{y} 3) / \mathrm{n}$-meanc (y2)*meanc (y3));

sigma1 $=(\operatorname{varc}(\mathrm{y} 1)-((\operatorname{sumc}(\mathrm{y} 1 . * \mathrm{y} 2) / \mathrm{n}-\operatorname{meanc}(\mathrm{y} 1) * \operatorname{meanc}(\mathrm{y} 2)) *$

$(\operatorname{sumc}(\mathrm{y} 1 . * \mathrm{y} 3) / \mathrm{n}$-meanc (y1)*meanc $(\mathrm{y} 3))) /(\operatorname{sumc}(\mathrm{y} 2 . * \mathrm{y} 3) / \mathrm{n}-$ meanc $(\mathrm{y} 2) * \operatorname{meanc}(\mathrm{y} 3)))$;

sigma2 $=(\operatorname{varc}(\mathrm{y} 2)-((\operatorname{sumc}(\mathrm{y} 2 \cdot * \mathrm{y} 3) / \mathrm{n}-\operatorname{meanc}(\mathrm{y} 2) *$ meanc $(\mathrm{y} 3)) *$

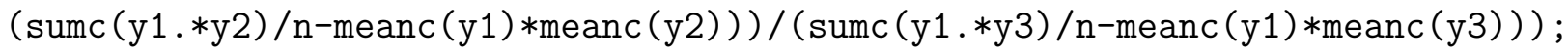

sigma3 $=(\operatorname{varc}(y 3)-((\operatorname{sumc}($ y2 . *y3) $/ \mathrm{n}$-meanc $(y 2) *$ meanc $(y 3)) *$

$(\operatorname{sumc}(\mathrm{y} 1 . * \mathrm{y} 3) / \mathrm{n}$-meanc (y1)*meanc $(\mathrm{y} 3))) /(\operatorname{sumc}(\mathrm{y} 1 . * \mathrm{y} 2) / \mathrm{n}$-meanc $(\mathrm{y} 1) *$ meanc $(\mathrm{y} 2)))$;

vsigma=sigma1 $\sim$ sigma2 ${ }^{\sim}$ sigma3;

//impressao dos valores iniciais para os estimadores

println ("sigma2x", sigma2x);

println("sigma1", sigma1);

println("sigma2", sigma2);

println("sigma3", sigma3);

println ("mix", mix);

println("alfa1",alfa1);

println("alfa2", alfa2);

println("alfa3", alfa3);

println("beta1", beta1);

println("beta2", beta2);

println ("beta3", beta3);

decl c, conv, $\mathrm{i}, \mathrm{j}$, cont $, \mathrm{xj}, \mathrm{xj} 2$;

conv $=100$;

$i=0$; while ( $i<$ conv)

\{

//passo E 


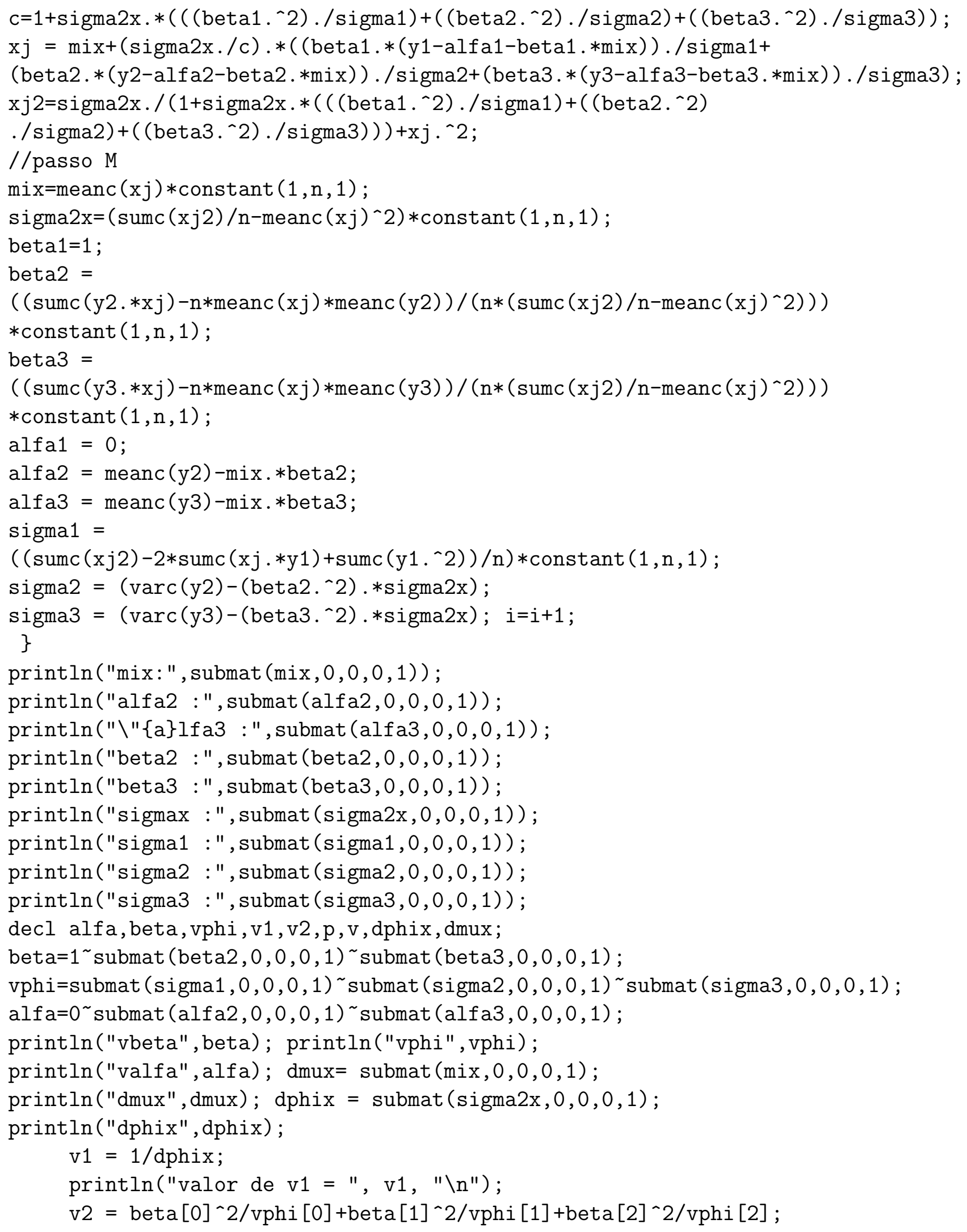




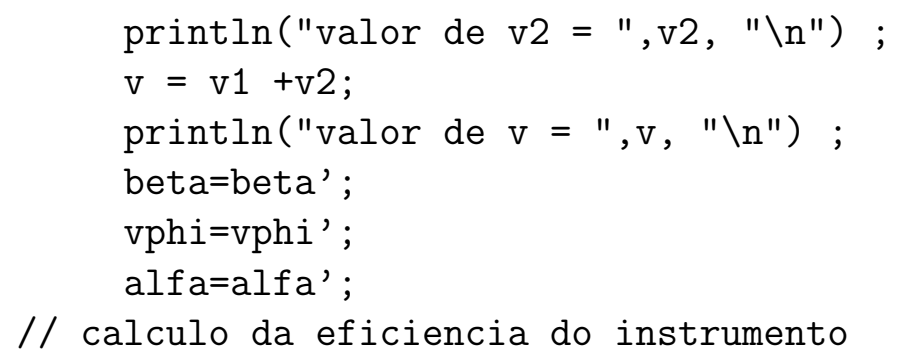


Ialfakbetak $=-$ num*beta $[1:] . * \operatorname{beta}[1:] * \operatorname{dmux} . /(\operatorname{v} * \operatorname{vph} i[1:] * * \operatorname{vphi}[1:])$ +num*dmux./(vphi [1:]);

for $(i=0 ; i<p ;++i)$

Ialfakbetal [i] [i] = Ialfakbetak[i];

Ibetakbetak $=\left(\right.$ num* $\left.*\left(\operatorname{dmux}^{\wedge} 2+\operatorname{dphix}-1 / v\right)\right) . / \operatorname{vphi}[1:]+($ num $* \operatorname{beta}[1:]$.

*beta $\left.[1:] *\left(2 / \mathrm{v}-\operatorname{dphix}-\operatorname{dmux}^{\wedge} 2\right)\right)$./(v*vphi [1:] .*vphi [1:]);

Ibetakbetal $=$ num* $\left(\operatorname{beta}[1:] * \operatorname{beta}[1:]^{\prime}\right) . /\left(\mathrm{v} * \operatorname{vphi}[1:] * \operatorname{vphi}[1:]^{\prime}\right) *$

(2/v-dphix-dmux`2);

for $(i=0 ; i<p ;++i)$

Ibetakbetal [i] [i] = Ibetakbetak [i];

Ibetaksigmax $=($ num*beta $[1:] *(\operatorname{dphix}-1 / v)) . /\left(v^{2} * \operatorname{vphi}[1:] * \operatorname{dphix}{ }^{\wedge} 2\right)$;

Ibetaksigma1 $=-$ num $* \operatorname{beta}[1:] . /\left(v^{\wedge} 2 * \operatorname{vphi}[1:] * \operatorname{vphi}\left[0{ }^{\wedge} 2\right)\right.$;

Ibetaksigmak $=($ num $* \operatorname{beta}[1:] . /(\operatorname{v} * \operatorname{vphi}[1:] . * \operatorname{vphi}[1:])) . *$

(1-beta[1:].*beta[1:]./(v*vphi[1:]));

Ibetaksigmal $=-\operatorname{num} *\left(\operatorname{beta}[1:] * \operatorname{beta}[1:]^{\prime} \cdot{ }^{\wedge} 2\right) . /\left(\mathrm{v}^{\wedge} 2 * \operatorname{vphi}[1:]\right.$

* vphi[1:]'.`2);

for $(i=0 ; i<p ;++i)$

Ibetaksigmal [i] [i] = Ibetaksigmak [i];

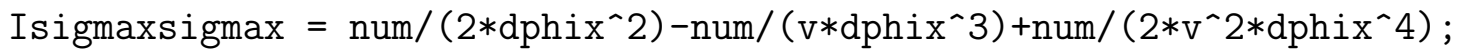

Isigmaxsigma1 $=$ num $/\left(2 * v^{\wedge} 2 * \operatorname{dphix}{ }^{\wedge} 2 * \operatorname{vphi}[0] \wedge 2\right)$;

Isigmaxsigmak $=$ num $*(\operatorname{beta}[1:] . * \operatorname{beta}[1:]) . /\left(2 * v^{\wedge} 2 * \operatorname{dphix}{ }^{\wedge} * \operatorname{vphi}[0]^{\wedge} 2\right)$;

Isigma1sigma1 = num/(2*vphi [0]^2)-num/(v*vphi [0]^3)+num/(2*v^2*vphi [0]^4);

Isigma1sigmak $=$ num* $(\operatorname{beta}[1:] . * \operatorname{beta}[1:]) . /\left(2 * \mathrm{v}^{\wedge} 2 * \operatorname{vphi}[0]^{\wedge} 2 * \operatorname{vphi}\left[1:{ }^{\wedge}{ }^{\wedge} 2\right)\right.$;

Isigmaksigmak $=$ num. $/(2 * \operatorname{vphi}[1:] . * \operatorname{vphi}[1:])-\operatorname{num} *(\operatorname{beta}[1:]$.

*beta[1:]) ./(v*vphi [1:] .*vphi [1:] .*vphi [1:])+num*(beta[1:] .*beta[1:]

* beta $[1:]$.

*beta[1:])./(2*v^2*vphi [1:] .*vphi [1:] **vphi [1:] **vphi [1:]);

Isigmaksigmal $=$ num* $(\operatorname{beta}[1:] . \wedge 2) *(\operatorname{beta}[1:] . \wedge 2)^{\prime} \cdot /\left(2 * \mathrm{v}^{\wedge} 2 *(\operatorname{vphi}[1:] . \wedge 2)\right.$

*(vphi $\left.\left.[1:] .{ }^{\wedge} 2\right)^{\prime}\right)$;

for $(i=0 ; i<p ;++i)$

Isigmaksigmal [i] [i] = Isigmaksigmak [i];

// concatena $\backslash c\{c\} \backslash \sim\{a\} o$ das submatrizes

decl Imix, Ialfa, Ibeta, Isigmax, Isigma1, Isigma;

Imix = Imixmix | Imixalfak | Imixbetak | Imixsigmax | Imixsigma1

I Imixsigmak;

Ialfa = Imixalfak'|Ialfakalfal|Ialfakbetal|Ialfaksigmax'|Ialfaksigma1'

I Ialfaksigmal;

Ibeta = Imixbetak'|Ialfakbetal'|Ibetakbetal $\mid$ Ibetaksigmax' $\mid$ Ibetaksigma1'

I Ibetaksigmal';

Isigmax = Imixsigmax $\mid$ Ialfaksigmax | Ibetaksigmax | Isigmaxsigmax |

Isigmaxsigma1 | Isigmaxsigmak;

Isigma1 = Imixsigma1 | Ialfaksigma1 | Ibetaksigma1 | Isigmaxsigma1 |

Isigma1sigma1 | Isigma1sigmak; 
Isigma = Imixsigmak'| Ialfaksigmal | Ibetaksigmal | Isigmaxsigmak' | Isigma1sigmak' | Isigmaksigmal;

// matriz de informa $\backslash c\{c\} \backslash \sim\{a\} 0$ de fisher decl I;

$I=\operatorname{Imix} \sim$ Ialfa $\sim$ Ibeta $\sim$ Isigmax $\sim$ Isigma1 $\sim$ Isigma; println("valor de $\mathrm{I}=$ ", I, "\n") ;

// matriz inversa da informa $\backslash c\{c\} \backslash \sim\{a\} 0$ de fisher

decl Inv;

$\operatorname{Inv}=\operatorname{invert}(\mathrm{I})$;

println("valor de Inv = ", Inv, "\n") ;

decl C, C1, C2, C3, W, b, W2, b2;

$\mathrm{b}=<0$;

0

1

$1>$;

$\mathrm{C}=<0,1,0,0,0,0,0,0,0$

$0,0,1,0,0,0,0,0,0$

$0,0,0,1,0,0,0,0,0$;

$0,0,0,0,1,0,0,0,0>$;

teta $=\operatorname{dmux} \mid$ alfa[1:] | beta[1:] | dphix | vphi;

// Estatistica de Wald para testar a seguinte hipotese: alfa2 = alfa3

// alfa2=alfa3 = alfa e beta2 = beta3 = beta

$\mathrm{W}=(\mathrm{C} * \text { teta }-\mathrm{b})^{\prime} *$ invert $\left(\mathrm{C} * \operatorname{Inv} * \mathrm{C}^{\prime}\right) *(\mathrm{C} *$ teta $-\mathrm{b})$;

println(" valor de C*teta-b ",C*teta-b);

println(" valor de invert $\left(C * \operatorname{Inv} * C^{\prime}\right)$ ", invert $\left.\left(C * \operatorname{Inv} * C^{\prime}\right)\right)$;

println("valor de $\mathrm{W}=$ ", $\mathrm{W}, " \backslash \mathrm{n}$ ") ;

$\mathrm{b} 2=<0$;

$0>$;

$\mathrm{C} 1=<0,1,-1,0,0,0,0,0,0$

$0,0,0,1,-1,0,0,0,0>$;

teta $=\operatorname{dmux} \mid$ alfa[1:] | beta[1:] | dphix | vphi;

// println("valor de teta = ", teta, "\n") ;

// Estatistica de Wald para testar a seguinte hipotese:

alfa2 = alfa3 $=0$

$/ /$ e beta2 $=$ beta3 $=1$

$\mathrm{W} 2=\left(\mathrm{C} 1 *\right.$ teta-b2)'*invert $\left(\mathrm{C} 1 * \operatorname{Inv} * \mathrm{C} 11^{\prime}\right) *(\mathrm{C} 1 *$ teta $-\mathrm{b} 2) ;$

println(" valor de C1*teta-b2 ",C1*teta-b2);

println(" valor de invert $(\mathrm{C} 1 * \operatorname{Inv} * \mathrm{C} 1$ ') ", invert $(\mathrm{C} 1 * \operatorname{Inv} * \mathrm{C} 1$ ')) ;

println("valor de $\mathrm{W} 2=$ ", W2, "\n");

$/ / \operatorname{println}(" \mathrm{~W} 1=$ ", $\mathrm{W}, \mathrm{W} 2=$ = , W2, "\n");

\}

É importante salientar que para os casos com 4 e 5 instrumentos foram feitas 
expansões do programa mostrado acima para 3 instrumentos. 


\section{Referências Bibliográficas}

[1] ALFARO, Katia Hermelinda Garcia, (2000) Modelo de Calibração Comparativa em Grupos, Tese, IME-USP

[2] AYRES JR., Frank (1976), Matrizes, Coleção Schaum, Editora McGRAW HILL DO BRASIL LTDA.

[3] BARNETT, V. D., Simultaneous pairwise linear structural relationships, Biometrics, 25 (1969), pp. 129-142.

[4] BICKEL, Peter Jonh e DOKSUM, Kjelli A. (1977), Mathematical Statistical San Francisco, Holden Day - USA.

[5] BOLFARINE, Heleno e ROJAS, Manuel Galea, (1995), Maximum Likelihood Estimation of Simultaneous Pairwise Linear Structural Relationships, Biometrical Journal, 37, pp. 673-689.

[6] CASElla, GEORGE e BERGER, ROGER L. (1990), Statistical Inference, Wadsworth Brooks, California, EUA

[7] CARTER, R., L. (1981), Restricted Maximum Likelihood Estimation of Bias and Reliability in the Comparison of Several Measuring Methods Biometrics, 37, pp. 733-741.

[8] DEMPSTER, A. P. N. M. and RUBIN. D. B. (1977), Maximum Likelihood from Incomplete Data via the EM Algorithm. Journal Roayl Statistical Society Ser B 39, pp. $1-38$

[9] DORNIK, J. A. (1996). Ox An Objeted Oriented Matrix Program Language, International Thomson Business Press., IP. London

[10] DORNIK, J. A. (1998). Objeted oriented matrix program using Ox 2.0. Timberlake Consultants Ltd, London

[11] FULLER, W. A., (1987), Measurement Error Models, Wiley, New York

[12] GRAYBILL, F. A. (1983), Matrices with applications in statistics. Second Edition, Wadsworth. 
[13] GILL, Philip E.; MURRAY, Walter e WRIGHT, Margaret, H. (1981), Practical Optimization, Academic Press, New York, EUA.

[14] JOHNSON, Richard A. e WICHERN, DEAN W.(1998), Applied Multivariate statistical Analysis, Fourth Edition, Prentice Hall, Upper saddle River, New Jersey EUA

[15] KAPLAN, WILFRED (1985). Cálculo Avançado, volume 1, quinta edição, Editora Edgard Blucher Ltda, Rio de Janeiro Brasil

[16] NETER, Jonh, KUTNER, Michael H., NACHTSHEIM, Christopher J. e WASSERMAN, William (1996), Applied Linear statistical Models, Fourth Edition, Irwin, Chicago, EUA

[17] NETER, Jonh, WASSERMAN, William e WHITMORE, G.A., (1993), Applied Statistics, Fourth Edition, Allyn and Bacon, Boston, EUA

[18] RAO, Calyampudi Radhakrishma (1973), Linear Statistifcal Inference and Its Aplications, Second Edition, Jonh Wiley \& Sons, New York, EUA.

[19] SIMÕES, João M., MELLO, Helladio do A. e BARBIN, Décio. (1967), Eficiência dos Aparelhos e Influência do Operador na Medição de altura Total das Árvores, O solo, 2, pp. $57-63$ 